RIMS-1758

INTER-UNIVERSAL TEICHMÜLLER THEORY III:

CANONICAL SPLITTINGS OF THE LOG-THETA-LATTICE

By

Shinichi MOCHIZUKI

August 2012

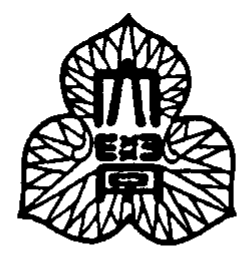

京都大学 数理解析研究所

RESEARCH INSTITUTE FOR MATHEMATICAL SCIENCES

KYOTO UNIVERSITY, Kyoto, Japan 


\title{
INTER-UNIVERSAL TEICHMÜLLER THEORY III: CANONICAL SPLITTINGS OF THE LOG-THETA-LATTICE
}

\author{
SHINichi Mochizuki
}

August 2012

\begin{abstract}
.
In the present paper, which is the third in a series of four papers, we study the theory surrounding the log-theta-lattice, a highly noncommutative two-dimensional diagram of "miniature models of conventional scheme theory", called $\Theta^{ \pm \text {ell } N F-H o d g e ~ t h e a t e r s, ~ t h a t ~ w e r e ~ a s s o c i a t e d, ~ i n ~ t h e ~ f i r s t ~ p a p e r ~ o f ~}$ the series, to certain data, called initial $\Theta$-data, that includes an elliptic curve $E_{F}$ over a number field $F$, together with a prime number $l \geq 5$. The horizontal arrows of the log-theta-lattice are defined as certain versions of the " $\Theta$-link" that was constructed, in the second paper of the series, by applying the theory of Hodge-Arakelovtheoretic evaluation - i.e., evaluation in the style of the scheme-theoretic HodgeArakelov theory established by the author in previous papers - of the [reciprocal of the $l$-th root of the] theta function at $\boldsymbol{l}$-torsion points. In the present paper, we study the theory surrounding the log-link between $\Theta^{ \pm \text {ell }}$ NF-Hodge theaters. The log-link is obtained, roughly speaking, by applying, at each [say, for simplicity, nonarchimedean] valuation of the number field under consideration, the local $p$-adic logarithm. The significance of the log-link lies in the fact it allows one to construct log-shells, i.e., roughly speaking, slightly adjusted forms of the image of the local units at the valuation under consideration via the local $p$-adic logarithm. The theory of log-shells was studied extensively in a previous paper of the author. The vertical arrows of the log-theta-lattice are given by the log-link. Consideration of various properties of the log-theta-lattice leads naturally to the establishment of multiradial algorithms for constructing "splitting monoids of logarithmic Gaussian procession monoids". Here, we recall that "multiradial algorithms" are algorithms that make sense from the point of view of an "alien arithmetic holomorphic structure", i.e., the ring/scheme structure of a $\Theta^{ \pm \text {ell }} \mathrm{NF}-H o d g e$ theater related to a given $\Theta^{ \pm \text {ell }} \mathrm{NF}$-Hodge theater by means of a non-ring/scheme-theoretic horizontal arrow of the log-theta-lattice. These logarithmic Gaussian procession monoids, or LGP-monoids, for short, may be thought of as the log-shell-theoretic versions of the Gaussian monoids that were studied in the second paper of the series. Finally, by applying these multiradial algorithms for splitting monoids of LGP-monoids, we obtain estimates for the log-volume of these LGP-monoids. These estimates will be applied to verify various diophantine results in the fourth paper of the series.
\end{abstract}

\section{Contents:}

Introduction

$\S 0$. Notations and Conventions

$\S 1$. The Log-theta-lattice

$\S 2$. Multiradial Theta Monoids

§3. Multiradial Logarithmic Gaussian Procession Monoids

Typeset by $\mathcal{A}_{\mathcal{M}} \mathcal{S}-\mathrm{T}_{\mathrm{EX}}$ 


\section{Introduction}

In the following discussion, we shall continue to use the notation of the Introduction to the first paper of the present series of papers [cf. [IUTchI], §I1]. In particular, we assume that are given an elliptic curve $E_{F}$ over a number field $F$, together with a prime number $l \geq 5$. In the first paper of the series, we introduced and studied the basic properties of $\Theta^{ \pm \text {ell }} N F$-Hodge theaters, which may be thought of as miniature models of the conventional scheme theory surrounding the given elliptic curve $E_{F}$ over the number field $F$. In the present paper, which forms the third paper of the series, we study the theory surrounding the log-link between $\Theta^{ \pm \text {ell }}$ NF-Hodge theaters. The log-link induces an isomorphism between the underlying $\mathcal{D}-\Theta^{ \pm \text {ell }} N F-H o d g e$ theaters and, roughly speaking, is obtained by applying, at each [say, for simplicity, nonarchimedean] valuation $\underline{v} \in \underline{\mathbb{V}}$, the local $p_{\underline{v}}$-adic logarithm to the local units [cf. Proposition 1.3, (i)]. The significance of the log-link lies in the fact it allows one to construct log-shells, i.e., roughly speaking, slightly adjusted forms of the image of the local units at $\underline{v} \in \underline{\mathbb{V}}$ via the local $p_{\underline{v}}$-adic logarithm. The theory of log-shells was studied extensively in [AbsTopIII]. The introduction of log-shells leads naturally to the construction of new versions - namely, the $\Theta_{\mathrm{LGP}}^{\times \boldsymbol{\mu}}-/ \Theta_{\mathfrak{l g p}}^{\times \boldsymbol{\mu}}$-links [cf. Definition 3.8, (ii)] — of the $\Theta-/ \Theta^{\times \boldsymbol{\mu}}-/ \Theta_{\mathrm{gau}}^{\times \boldsymbol{\mu}}$-links studied in [IUTchI], [IUTchII]. The resulting [highly non-commutative!] diagram of iterates of the log- [i.e., the vertical arrows] and $\Theta_{-} / \Theta^{\times \boldsymbol{\mu}_{-}} / \Theta_{\mathrm{gau}^{-}}^{\times \boldsymbol{\mu}^{-}} / \Theta_{\mathrm{LGP}^{-}}^{\times \boldsymbol{\mu}} / \Theta_{\mathfrak{l g p}}^{\times \boldsymbol{\mu}}$-links [i.e., the horizontal arrows] — which we refer to as the log-theta-lattice [cf. Definitions $1.4 ; 3.8$, (iii), as well as Fig. I.1 below, in the case of the $\Theta_{\mathrm{LGP}}^{\times \boldsymbol{\mu}}-$ link] — plays a central role in theory of the present series of papers.

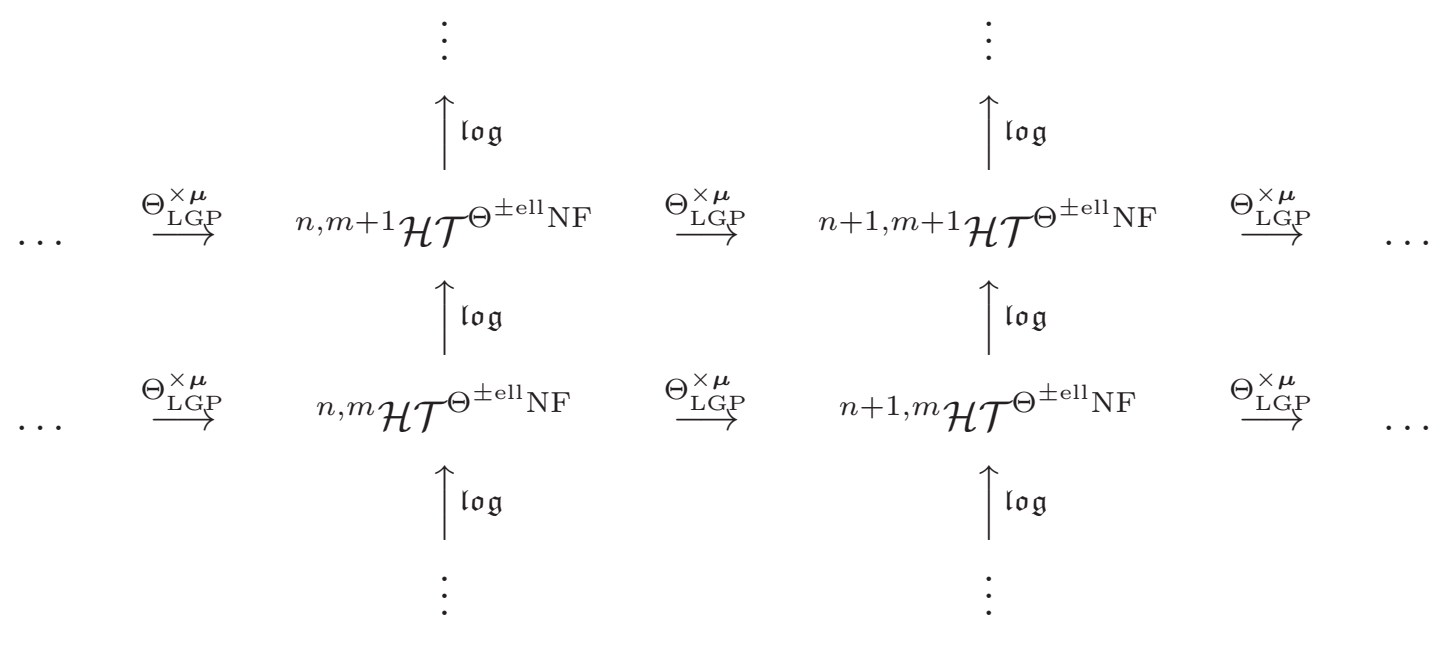

Fig. I.1: The [LGP-Gaussian] log-theta-lattice

Consideration of various properties of the log-theta-lattice leads naturally to the establishment of multiradial algorithms for constructing "splitting monoids of logarithmic Gaussian procession monoids" [cf. Theorem A below]. Here, we recall that "multiradial algorithms" [cf. the discussion of [IUTchII], Introduction] are algorithms that make sense from the point of view of an "alien arithmetic holomorphic structure", i.e., the ring/scheme structure of a $\Theta^{ \pm \text {ell }}$ NF-Hodge theater related to a given $\Theta^{ \pm \text {ell }} \mathrm{NF}$-Hodge theater by means of a non-ring/schemetheoretic $\Theta_{-} / \Theta^{\times \boldsymbol{\mu}_{-}} / \Theta_{\mathrm{gau}^{\times \boldsymbol{\mu}}}^{\times \boldsymbol{\mu}} / \Theta_{\mathrm{LGP}^{-}}^{\times \boldsymbol{\mu}} / \Theta_{\mathfrak{l g p}}^{\times \boldsymbol{\mu}}$-link. These logarithmic Gaussian procession 
monoids, or LGP-monoids, for short, may be thought of as the log-shell-theoretic versions of the Gaussian monoids that were studied in [IUTchII]. Finally, by applying these multiradial algorithms for splitting monoids of LGP-monoids, we obtain estimates for the log-volume of these LGP-monoids [cf. Theorem B below]. These estimates will be applied to verify various diophantine results in [IUTchIV].

Recall [cf. [IUTchI], §I1] the notion of an $\mathcal{F}$-prime-strip. An $\mathcal{F}$-prime-strip consists of data indexed by the valuations $\underline{v} \in \mathbb{V}$; roughly speaking, the data at each $\underline{v}$ consists of a Frobenioid, i.e., in essence, a system of monoids over a base category. For instance, at $\underline{v} \in \underline{\mathbb{V}}^{\text {bad }}$, this data may be thought of as an isomorphic copy of the monoid with Galois action

$$
\Pi_{\underline{v}} \curvearrowright \mathcal{O} \bar{F}_{\underline{v}}^{\triangleright}
$$

— where we recall that $\mathcal{O} \bar{F}_{v}$ denotes the multiplicative monoid of nonzero integral elements of the completion of an algebraic closure $\bar{F}$ of $F$ at a valuation lying over $\underline{v}$ [cf. [IUTchI], §I1, for more details]. The $p_{\underline{v}}$-adic logarithm $\log _{\underline{v}}: \mathcal{O}_{\bar{F}_{\underline{v}}}^{\times} \rightarrow \bar{F}_{\underline{v}}$ at $\underline{v}$ then defines a natural $\Pi_{\underline{\underline{v}}}$-equivariant isomorphism of topological modūles

$$
\left(\mathcal{O}_{\bar{F}_{\underline{v}}}^{\times \mu} \otimes \mathbb{Q} \stackrel{\sim}{\rightarrow}\right) \quad \mathcal{O}_{\bar{F}_{\underline{v}}}^{\times} \otimes \mathbb{Q} \stackrel{\sim}{\rightarrow} \bar{F}_{\underline{v}}
$$

— where we recall the notation " $\mathcal{O}_{\overline{F_{\underline{v}}}}^{\times \mu}=\mathcal{O}_{\bar{F}_{\underline{v}}}^{\times} / \mathcal{O}_{\overline{F_{\underline{v}}}}^{\mu}$ " from the discussion of [IUTchI], $\S 1$ - which allows one to equip $\mathcal{O}_{\overline{F^{v}}}^{\overline{\bar{x}}} \otimes \mathbb{Q}$ with the field structure arising from the field structure of $\bar{F}_{\underline{v}}$. The portion at $\underline{v}$ of the log-link associated to an $\mathcal{F}$-prime-strip [cf. Definition 1.1, (iii); Proposition 1.2] may be thought of as the correspondence

$$
\left\{\Pi_{\underline{v}} \curvearrowright \mathcal{O} \bar{F}_{\underline{v}}^{\triangleright}\right\} \quad \stackrel{\log }{\longrightarrow} \quad\left\{\Pi_{\underline{v}} \curvearrowright \mathcal{O} \bar{F}_{\underline{v}}^{\triangleright}\right\}
$$

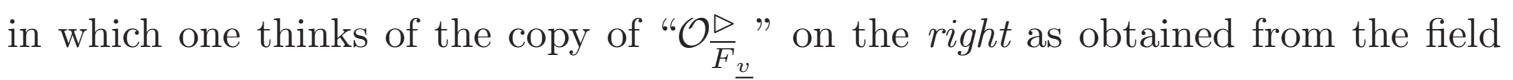
structure induced by the $p_{\underline{v}}$-adic logarithm on the tensor product with $\mathbb{Q}$ of the copy of the units " $\mathcal{O}_{\bar{F}_{\underline{v}}}^{\times} \subseteq \mathcal{O}_{\bar{F}_{\underline{v}}}^{\triangleright}$ " on the left. Since this correspondence induces an isomorphism of topological groups between the copies of $\Pi_{\underline{v}}$ on either side, one may think of $\Pi_{\underline{v}}$ as "immune to"/ "neutral with respect to" - or, in the terminology of the present series of papers, "coric" with respect to - the transformation constituted by the log-link. This situation is studied in detail in [AbsTopIII], $\S 3$, and reviewed in Proposition 1.2 of the present paper.

By applying various results from absolute anabelian geometry, one may algorithmically reconstruct a copy of the data " $\Pi_{\underline{v}} \curvearrowright \mathcal{O} \bar{F}_{\underline{v}}^{\triangleright}$ " from $\Pi_{\underline{v}}$. Moreover, by applying Kummer theory, one obtains natural isomorphisms between this "coric version" of the data " $\Pi_{\underline{v}} \curvearrowright \mathcal{O} \stackrel{\bar{F}}{\underline{v}}^{\triangleright}$ " and the copies of this data that appear on either side of the log-link. On the other hand, one verifies immediately that these Kummer isomorphisms are not compatible with the coricity of the copy of the data " $\Pi_{\underline{v}} \curvearrowright \mathcal{O} \bar{F}_{\underline{v}}$ " algorithmically constructed from $\Pi_{\underline{v}}$. This phenomenon is, in some sense, the central theme of the theory of [AbsTopIII], $\S 3$, and is reviewed in Proposition 1.2, (iv), of the present paper. 
The introduction of the log-link leads naturally to the construction of logshells at each $\underline{v} \in \underline{\mathbb{V}}$. If, for simplicity, $\underline{v} \in \underline{\mathbb{V}}^{\text {bad }}$, then the $\log$-shell at $\underline{v}$ is given, roughly speaking, by the compact additive module

$$
\mathcal{I}_{\underline{v}} \stackrel{\text { def }}{=} p_{\underline{v}}^{-1} \cdot \log _{\underline{v}}\left(\mathcal{O}_{K_{\underline{v}}}^{\times}\right) \subseteq K_{\underline{v}} \subseteq \bar{F}_{\underline{v}}
$$

[cf. Definition 1.1, (i), (ii); Remark 1.2.2, (i), (ii)]. One has natural functorial algorithms for constructing various versions - i.e., mono-analytic/holomorphic and étale-like/Frobenius-like — from $\mathcal{D}^{\vdash}-/ \mathcal{D}-/ \mathcal{F}^{\vdash}-/ \mathcal{F}$-prime-strips [cf. Proposition 1.2, (v), (vi), (vii), (viii), (ix)]. Although, as discussed above, the relevant Kummer isomorphisms are not compatible with the log-link "at the level of elements", the $\log$-shell $\mathcal{I}_{\underline{v}}$ at $\underline{v}$ satisfies the important property

$$
\mathcal{O}_{K_{\underline{v}}}^{\triangleright} \subseteq \mathcal{I}_{\underline{v}} ; \quad \log _{\underline{v}}\left(\mathcal{O}_{K_{\underline{v}}}^{\times}\right) \subseteq \mathcal{I}_{\underline{v}}
$$

- i.e., it contains the images of the Kummer isomorphisms associated to both the domain and the codomain of the log-link [cf. Proposition 1.2, (v); Remark 1.2.2, (i), (ii)]. In light of the compatibility of the log-link with log-volumes [cf. Propositions 1.2, (iii); 3.9, (iv)], this property will ultimately lead to upper bounds - i.e., as opposed to "precise equalities" — in the computation of log-volumes in Corollary 3.12 [cf. Theorem B below]. Put another way, although iterates of the log-link fail to be compatible with the various Kummer isomorphisms that arise, one may nevertheless consider the entire diagram that results from considering such iterates of the log-link and related Kummer isomorphisms [cf. Proposition 1.2, (x)]. We shall refer to such diagrams

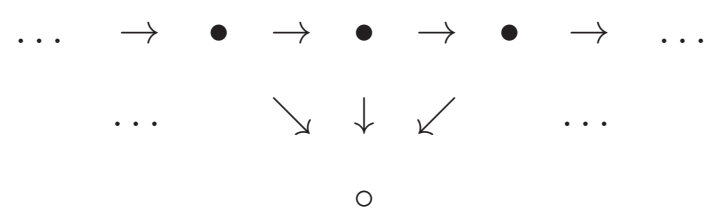

- i.e., where the horizontal arrows correspond to the log-links [that is to say, to the vertical arrows of the log-theta-lattice!]; the "•'s" correspond to the Frobenioidtheoretic data within a $\Theta^{ \pm \text {ell } N F-H o d g e ~ t h e a t e r ; ~ t h e ~ " o " ~ c o r r e s p o n d s ~ t o ~ t h e ~ c o r i c ~}$ version of this data [that is to say, in the terminology discussed below, vertically coric data of the log-theta-lattice]; the vertical/diagonal arrows correspond to the various Kummer isomorphisms - as log-Kummer correspondences [cf. Theorem 3.11, (ii); Theorem A, (ii), below]. Then the inclusions of the above display may be interpreted as a sort of "upper semi-commutativity" of such diagrams [cf. Remark 1.2.2, (iii)], which we shall also refer to as the "upper semicompatibility" of the log-link with the relevant Kummer isomorphisms - cf. the discussion of the "indeterminacy" (Ind3) in Theorem 3.11, (ii).

By considering the log-links associated to the various $\mathcal{F}$-prime-strips that occur in a $\Theta^{ \pm \text {ell }} N F$-Hodge theater, one obtains the notion of a log-link between $\Theta^{ \pm \text {ell }} N F$ Hodge theaters

$$
{ }^{\dagger} \mathcal{H} \mathcal{T}^{\Theta^{ \pm e l l} \mathrm{NF}} \stackrel{\text { log }}{\longrightarrow} \ddagger \mathcal{H} \mathcal{T}^{\Theta^{ \pm e l l} \mathrm{NF}}
$$

[cf. Proposition 1.3, (i)]. As discussed above, by considering the iterates of the $\mathfrak{l o g}$ [i.e., the vertical arrows] and $\Theta_{-} / \Theta^{\times \boldsymbol{\mu}_{-}} / \Theta_{\mathrm{gau}^{-}}^{\times \boldsymbol{\mu}_{-}} / \Theta_{\mathrm{LGP}^{-}}^{\times \boldsymbol{\mu}} / \Theta_{\mathfrak{l g p}}^{\times \boldsymbol{\mu}}$-links [i.e., the horizontal 
arrows], one obtains a diagram which we refer to as the log-theta-lattice [cf. Definitions 1.4; 3.8, (iii), as well as Fig. I.1, in the case of the $\Theta_{\mathrm{LGP}}^{\times \mu}$-link]. As discussed above, this diagram is highly noncommutative, since the definition of

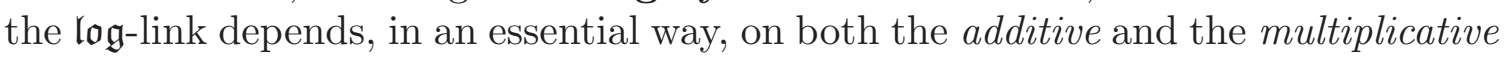
structures - i.e., on the ring structure - of the various local rings at $\underline{v} \in \underline{\mathbb{V}}$, structures which are not preserved by the $\Theta_{-} / \Theta^{\times \boldsymbol{\mu}_{-}} / \Theta_{\mathrm{gau}}^{\times \boldsymbol{\mu}}-/ \Theta_{\mathrm{LGP}}^{\times \boldsymbol{\mu}}-\Theta_{\mathfrak{l g p}}^{\times \boldsymbol{\mu}}$-links $[\mathrm{cf}$. Remark 1.4.1, (i)]. So far, in the Introductions to [IUTchI], [IUTchII], as well as in the present Introduction, we have discussed various "coricity" properties - i.e., properties of invariance with respect to various types of "transformations" - in the context of $\Theta_{-} / \Theta^{\times \boldsymbol{\mu}_{-}} / \Theta_{\mathrm{gau}^{-}}^{\times \boldsymbol{\mu}} / \Theta_{\mathrm{LGP}^{-}}^{\times \boldsymbol{\mu}} / \Theta_{\mathfrak{l g p}}^{\times \boldsymbol{\mu}}$-links, as well as in the context of log-links. In the context of the log-theta-lattice, it becomes necessary to distinguish between various types of coricity. That is to say, coricity with respect to log-links [i.e., the vertical arrows of the log-theta-lattice] will be referred to as vertical coricity, while coricity with respect to $\Theta_{-} / \Theta^{\times \boldsymbol{\mu}_{-}} / \Theta_{\mathrm{gau}^{-}}^{\times \boldsymbol{\mu}_{-}} / \Theta_{\mathrm{LGP}^{-}}^{\times \boldsymbol{\mu}} / \Theta_{\mathfrak{l g p}}^{\times \boldsymbol{\mu}}$-links [i.e., the horizontal arrows of the log-theta-lattice] will be referred to as horizontal coricity. On the other hand, coricity properties that hold with respect to all of the arrows of the log-theta-lattice will be referred to as bi-coricity properties.

Relative to the analogy between the theory of the present series of papers and p-adic Teichmüller theory [cf. [IUTchI], §I4], we recall that a $\Theta^{ \pm \text {ell }} N F$-Hodge theater, which may be thought of as a miniature model of the conventional scheme theory surrounding the given elliptic curve $E_{F}$ over the number field $F$, corresponds to the positive characteristic scheme theory surrounding a hyperbolic curve over a positive characteristic perfect field that is equipped with a nilpotent ordinary indigenous bundle [cf. Fig. I.2 below]. Then the rotation, or "juggling", effected by the log-link of the additive and multiplicative structures of the conventional scheme theory represented by a $\Theta^{ \pm \text {ell }} \mathrm{NF}$-Hodge theater may be thought of as corresponding to the Frobenius morphism in positive characteristic [cf. the discussion of [AbsTopIII], §I3, §I5]. Thus, just as the Frobenius morphism is completely welldefined in positive characteristic, the log-link may be thought of as a phenomenon that occurs within a single arithmetic holomorphic structure, i.e., a vertical line of the log-theta-lattice. By contrast, the essentially non-ring/scheme-theoretic relationship between $\Theta^{ \pm \text {ell }} \mathrm{NF}-$ Hodge theaters constituted by the $\Theta_{-} / \Theta^{\times \mu_{-}} / \Theta_{\mathrm{gau}^{-}}$ $/ \Theta_{\mathrm{LGP}^{-}}^{\times \boldsymbol{\mu}} / \Theta_{\mathfrak{l g p}^{\times}}^{\times \boldsymbol{\mu}}$-links corresponds to the relationship between the "mod $p^{n}$ " and "mod $p^{n+1}$ " portions of the ring of Witt vectors, in the context of a canonical lifting of the original positive characteristic data [cf. the discussion of Remark 1.4.1, (iii); Fig. I. 2 below]. Thus, the log-theta-lattice, taken as a whole, may be thought of as corresponding to the canonical lifting of the original positive characteristic data, equipped with a corresponding canonical Frobenius action/lifting [cf. Fig. I.2 below]. Finally, the non-commutativity of the log-theta-lattice may be thought of as corresponding to the complicated "intertwining" that occurs in the theory of Witt vectors and canonical liftings between the Frobenius morphism in positive characteristic and the mixed characteristic nature of the ring of Witt vectors [cf. the discussion of Remark 1.4.1, (ii), (iii)].

One important consequence of this "noncommutative intertwining" of the two dimensions of the log-theta-lattice is the following. Since each horizontal arrow of the log-theta-lattice [i.e., the $\Theta_{-} / \Theta^{\times \mu_{-}} / \Theta_{\text {gau }^{-}}^{\times \mu} / \Theta_{\mathrm{LGP}^{-}}^{\times \boldsymbol{\mu}} / \Theta_{\mathfrak{l g p}}^{\times \boldsymbol{\mu}}-\mathrm{link}$ ] may only be used to relate - i.e., via various Frobenioids - the multiplicative portions of the 
ring structures in the domain and codomain of the arrow, one natural approach to relating the additive portions of these ring structures is to apply the theory of log-shells. That is to say, since each horizontal arrow is compatible with the canonical splittings [up to roots of unity] discussed in [IUTchII], Introduction, of the theta/Gaussian monoids in the domain of the horizontal arrow into unit group and value group portions, it is natural to attempt to relate the ring structures on either side of the horizontal arrow by applying the canonical splittings to

- relate the multiplicative structures on either side of the horizontal arrow by means of the value group portions of the theta/Gaussian monoids;

- relate the additive structures on either side of the horizontal arrow by means of the unit group portions of the theta/Gaussian monoids, shifted once via a vertical arrow, i.e., the log-link, so as to "render additive" the [a priori $]$ multiplicative structure of these unit group portions.

Indeed, this is the approach that will ultimately be taken in Theorem 3.11 [cf. Theorem A below] to relating the ring structures on either side of a horizontal arrow. On the other hand, in order to actually implement this approach, it will be necessary to overcome numerous technical obstacles. Perhaps the most immediately obvious such obstacle lies in the observation [cf. the discussion of Remark 1.4.1, (ii)] that, precisely because of the "noncommutative intertwining" nature of the log-theta-lattice,

any sort of algorithmic construction concerning objects lying in the $d o$ main of a horizontal arrow that involves vertical shifts [e.g., such as the approach to relating additive structures in the fashion described above] cannot be "translated" in any immediate sense into an algorithm that makes sense from the point of view of the codomain of the horizontal arrow.

In a word, our approach to overcoming this technical obstacle consists of working with objects in the vertical line of the log-theta-lattice that contains the domain of the horizontal arrow under consideration that satisfy the crucial property of being

\section{invariant with respect to vertical shifts}

- i.e., shifts via iterates of the log-link [cf. the discussion of Remarks 1.2.2; 1.4.1, (ii)]. For instance, étale-like objects that are vertically coric satisfy this invariance property. On the other hand, as discussed in the beginning of [IUTchII], Introduction, in the theory of the present series of papers, it is of crucial importance to be able to relate corresponding Frobenius-like and étale-like structures to one another via Kummer theory. In particular, in order to obtain structures that are invariant with respect to vertical shifts, it is necessary to consider log-Kummer correspondences, as discussed above. Moreover, in the context of such log-Kummer correspondences, typically, one may only obtain structures that are invariant with respect to vertical shifts if one is willing to admit some sort of indeterminacy, e.g., such as the "upper semi-compatibility" [cf. the discussion of the "indeterminacy" (Ind3) in Theorem 3.11, (ii)] discussed above. 


\begin{tabular}{|c|c|}
\hline Inter-universal Teichmüller theory & p-adic Teichmüller theory \\
\hline $\begin{array}{c}\text { number field } \\
F\end{array}$ & $\begin{array}{c}\text { hyperbolic curve } C \text { over a } \\
\text { positive characteristic perfect field }\end{array}$ \\
\hline $\begin{array}{c}\text { [once-punctured] } \\
\text { elliptic curve } \\
X \text { over } F\end{array}$ & $\begin{array}{c}\text { nilpotent ordinary } \\
\text { indigenous bundle } \\
P \text { over } C\end{array}$ \\
\hline $\begin{array}{l}\Theta \text {-link arrows of the } \\
\text { log-theta-lattice }\end{array}$ & $\begin{array}{l}\text { mixed characteristic extension } \\
\text { structure of a ring of Witt vectors }\end{array}$ \\
\hline $\begin{array}{l}\text { log-link arrows of the } \\
\text { log-theta-lattice }\end{array}$ & $\begin{array}{l}\text { the Frobenius morphism } \\
\text { in positive characteristic }\end{array}$ \\
\hline $\begin{array}{l}\text { the entire } \\
\text { log-theta-lattice }\end{array}$ & $\begin{array}{c}\text { the resulting canonical lifting } \\
+ \text { canonical Frobenius action; } \\
\text { canonical Frobenius lifting } \\
\text { over the ordinary locus }\end{array}$ \\
\hline $\begin{array}{c}\text { relatively straightforward } \\
\text { original construction of } \\
\Theta_{\mathrm{LGP}}^{\times \mu}-\text { link }\end{array}$ & $\begin{array}{l}\text { relatively straightforward } \\
\text { original construction of } \\
\text { canonical liftings }\end{array}$ \\
\hline $\begin{array}{l}\text { highly nontrivial } \\
\text { description of alien arithmetic } \\
\text { holomorphic structure } \\
\text { via absolute anabelian geometry }\end{array}$ & $\begin{array}{l}\text { highly nontrivial } \\
\text { absolute anabelian } \\
\text { reconstruction of } \\
\text { canonical liftings }\end{array}$ \\
\hline
\end{tabular}

Fig. I.2: Correspondence between inter-universal Teichmüller theory and p-adic Teichmüller theory

One important property of the log-link, and hence, in particular, of the construction of log-shells, is its compatibility with the $\mathbb{F}_{l}^{\rtimes \pm}$-symmetry discussed in the Introductions to [IUTchI], [IUTchII] — cf. Remark 1.3.2. Here, we recall from the discussion of [IUTchII], Introduction, that the $\mathbb{F}_{l}^{\rtimes \pm}$-symmetry allows one to relate the various $\mathcal{F}$-prime-strips - i.e., more concretely, the various copies of the 
data " $\Pi_{\underline{v}} \curvearrowright \mathcal{O} \bar{F}_{\underline{v}}$ " at $\underline{v} \in \underline{\mathbb{V}}^{\text {bad }}$ [and their analogues for $\underline{v} \in \underline{\mathbb{V}}^{\text {good }}$ ] - associated to the various labels $\in \mathbb{F}_{l}$ that appear in the Hodge-Arakelov-theoretic evaluation of [IUTchII] in a fashion that is compatible with

- the distinct nature of distinct labels $\in \mathbb{F}_{l}$;

- the Kummer isomorphisms used to relate Frobenius-like and étalelike versions of the $\mathcal{F}$-prime-strips appear, i.e., more concretely, the various copies of the data " $\Pi_{\underline{v}} \curvearrowright \mathcal{O} \bar{F}_{\underline{v}}^{\triangleright}$ " at $\underline{v} \in \underline{\mathbb{V}}^{\text {bad }}$ [and their analogues for $\left.\underline{v} \in \underline{\mathbb{V}}^{\text {good }}\right]$

- the structure of the underlying $\mathcal{D}$-prime-strips that appear, i.e., more concretely, the various copies of the [arithmetic] tempered fundamental group " $\Pi_{\underline{v}}$ " at $\underline{v} \in \underline{\mathbb{V}}^{\text {bad }}$ [and their analogues for $\left.\underline{v} \in \underline{\mathbb{V}}^{\text {good }}\right]$

— cf. the discussion of [IUTchII], Introduction; Remark 1.5.1, Step (vii) of the proof of Corollary 3.12 of the present paper. This compatibility with the $\mathbb{F}_{l}^{\rtimes \pm}$-symmetry gives rise to the construction of

• "arithmetically holomorphic" $\mathcal{F}^{\vdash \times \mu}$-prime strips, log-shells which are vertically coric;

- mono-analytic $\mathcal{F}^{\vdash \times \boldsymbol{\mu}}$-prime strips, log-shells which are bi-coric

- cf. Theorem 1.5. These bi-coric mono-analytic log-shells play a central role in the theory of the present paper.

One notable aspect of the compatibility of the log-link with the $\mathbb{F}_{l}^{\rtimes \pm}$-symmetry in the context of the theory of Hodge-Arakelov-theoretic evaluation developed in [IUTchII] is the following. One important property of mono-theta environments is the property of "isomorphism class compatibility", i.e., in the terminology of [EtTh], "compatibility with the topology of the tempered fundamental group" [cf. the discussion of Remark 2.1.1]. This "isomorphism class compatibility" allows one to apply the Kummer theory of mono-theta environments [i.e., the theory of [EtTh]] relative to the ring-theoretic basepoints that occur on either side of the log-link [cf. Remark 2.1.1, (ii); [IUTchII], Remark 3.6.4, (i)], for instance, in the context of the log-Kummer correspondences discussed above. Here, we recall that the significance of working with such "ring-theoretic basepoints" lies in the fact that the full ring structure of the local rings involved [i.e., as opposed to, say, just the multiplicative portion of this ring structure] is necessary in order to construct the log-link. That is to say, it is precisely by establishing the conjugate synchronization arising from the $\mathbb{F}_{l}^{\rtimes \pm}$-symmetry relative to these basepoints that occur on either side of the log-link that one is able to conclude the crucial compatibility of this conjugate synchronization with the log-link discussed in Remark 1.3.2. Thus, in summary, one important consequence of the "isomorphism class compatibility" of mono-theta environments is the simultaneous compatibility of

- the Kummer theory of mono-theta environments;

. the conjugate synchronization arising from the $\mathbb{F}_{l}^{\rtimes \pm}$-symmetry;

- the construction of the log-link. 
This simultaneous compatibility is necessary in order to perform the construction of the [crucial!] splitting monoids of LGP-monoids referred to above - cf. the discussion of Step (vi) of the proof of Corollary 3.12.

In $\S 2$ of the present paper, we continue our preparation for the multiradial construction of splitting monoids of LGP-monoids given in $\S 3$ [of the present paper] by presenting a global formulation of the essentially local theory at $\underline{v} \in \underline{\mathbb{V}}^{\text {bad }}[\mathrm{cf}$. [IUTchII], $§ 1, \S 2, \S 3]$ concerning the interpretation, via the notion of multiradiality, of various ridigity properties of mono-theta environments. That is to say, although much of the [essentially routine!] task of formulating the local theory of [IUTchII], $1, \S 2, \S 3$, in global terms was accomplished in [IUTchII], $\S 4$, the [again essentially routine!] task of formulating the portion of this local theory that concerns multiradiality was not addressed in the theory of [IUTchII], $§ 4$. One reason for this lies in the fact that, from the point of view of the theory to be developed in $\S 3$ of the present paper, this global formulation of multiradiality properties of the monotheta environment may be presented most naturally in the framework developed in $\S 1$ of the present paper, involving the log-theta-lattice [cf. Theorem 2.2; Corollary 2.3]. Indeed, the étale-like versions of the mono-theta environment, as well as the various objects constructed from the mono-theta environment, may be interpreted, from the point of view of the log-theta-lattice, as vertically coric structures, and are Kummer-theoretically related to their Frobenius-like [i.e., Frobenioidtheoretic] counterparts, which arise from the [Frobenioid-theoretic portions of the] various $\Theta^{ \pm \text {ell }} \mathrm{NF}$-Hodge theaters in a vertical line of the log-theta-lattice [cf. Theorem 2.2, (ii); Corollary 2.3, (ii), (iii), (iv)]. Moreover, it is precisely the horizontal arrows of the log-theta-lattice that give rise to the $\widehat{\mathbb{Z}}^{\times}$-indeterminacies acting on copies of " $\mathcal{O}^{\times \boldsymbol{\mu}}$ " that play a prominent role in the local multiradiality theory developed in [IUTchII] [cf. the discussion of [IUTchII], Introduction]. In this context, it is useful to recall from the discussion of [IUTchII], Introduction [cf. also Remark 2.2.1 of the present paper], that the essential content of this local multiradiality theory consists of the observation [cf. Fig. I.3 below] that, since mono-theta-theoretic cyclotomic and constant multiple rigidity only require the use of the portion of $\mathcal{O}_{\bar{F}_{v}}^{\times}$, for $\underline{v} \in \underline{\mathbb{V}}^{\text {bad }}$, given by the torsion subgroup $\mathcal{O}_{\bar{F}_{\underline{v}}}^{\mu} \subseteq \mathcal{O}_{\bar{F}_{\underline{v}}}^{\times}$[i.e., the roots of unity], the triviality of the composite of natural morphisms

$$
\mathcal{O}_{\bar{F}_{\underline{v}}}^{\mu} \hookrightarrow \mathcal{O}_{\bar{F}_{\underline{v}}}^{\times} \rightarrow \mathcal{O}_{\bar{F}_{\underline{v}}}^{\times \mu}
$$

has the effect of insulating the Kummer theory of the étale theta function - i.e., via the theory of the mono-theta environments developed in [EtTh] — from the $\widehat{\mathbb{Z}}^{\times}$-indeterminacies that act on the copies of "OOำ prime-strips that appear in the $\Theta_{-} / \Theta^{\times \boldsymbol{\mu}_{-}} / \Theta_{\mathrm{gau}^{\times \boldsymbol{\mu}}-} / \Theta_{\mathrm{LGP}}^{\times \boldsymbol{\mu}}-/ \Theta_{\mathfrak{l g p}}^{\times \boldsymbol{\mu}}-$ link.

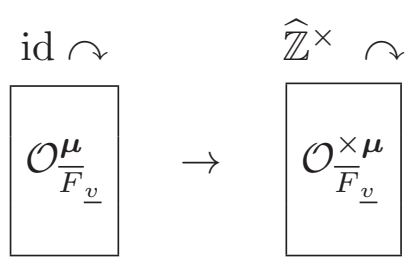

Fig. I.3: Insulation from $\widehat{\mathbb{Z}}^{\times}$-indeterminacies in the context of mono-theta-theoretic cyclotomic, constant multiple rigidity 
In $\S 3$ of the present paper, which, in some sense, constitutes the conclusion of the theory developed thus far in the present series of papers, we present the construction of the [splitting monoids of] LGP-monoids, which may be thought of as a multiradial version of the [splitting monoids of] Gaussian monoids that were constructed via the theory of Hodge-Arakelov-theoretic evaluation developed in [IUTchII]. In order to achieve this multiradiality, it is necessary to "multiradialize" the various components of the construction of the Gaussian monoids given in [IUTchII]. The first step in this process of "multiradialization" concerns the labels $j \in \mathbb{F}_{i}^{*}$ that occur in the Hodge-Arakelov-theoretic evaluation performed in [IUTchII]. That is to say, the construction of these labels, together with the closely related theory of $\mathbb{F}_{l}^{*}$-symmetry, depend, in an essential way, on the full arithmetic tempered fundamental groups " $\Pi_{\underline{v}}$ " at $\underline{v} \in \underline{\mathbb{V}}^{\text {bad }}$, i.e., on the portion of the arithmetic holomorphic structure within a $\Theta^{ \pm \text {ell }} \mathrm{NF}-$ Hodge theater which is not shared by an alien arithmetic holomorphic structure [i.e., an arithmetic holomorphic structure related to the original arithmetic holomorphic structure via a horizontal arrow of the log-theta-lattice]. One naive approach to remedying this state of affairs is to simply consider the underlying set, of cardinality $l^{*}$, associated to $\mathbb{F}_{l}^{*}$, which we regard as being equipped with the full set of symmetries given by arbitrary permutation automorphisms of this underlying set. The problem with this approach is that it yields a situation in which, for each label $j \in \mathbb{F}_{l}^{*}$, one must contend with an indeterminacy of $l$ * possibilities for the element of this underlying set that corresponds to $j$ [cf. [IUTchI], Propositions 4.11, (i); 6.9, (i)]. From the point of view of the log-volume computations to be performed in [IUTchIV], this degree of indeterminacy gives rise to log-volumes which are "too large", i.e., to estimates that are not sufficient for deriving the various diophantine results obtained in [IUTchIV]. Thus, we consider the following alternative approach, via processions [cf. [IUTchI], Propositions, 4.11, 6.9]. Instead of working just with the underlying set associated to $\mathbb{F}_{l}^{*}$, we consider the diagram of inclusions of finite sets

$$
\mathbb{S}_{1}^{ \pm} \hookrightarrow \mathbb{S}_{1+1=2}^{ \pm} \quad \hookrightarrow \quad \ldots \quad \hookrightarrow \quad \mathbb{S}_{j+1}^{ \pm} \quad \hookrightarrow \quad \ldots \quad \hookrightarrow \quad \mathbb{S}_{1+l^{*}=l^{ \pm}}^{ \pm}
$$

— where we write $\mathbb{S}_{j+1}^{+} \stackrel{\text { def }}{=}\{0,1, \ldots, j\}$, for $j=0, \ldots, l^{*}$, and we think of each of these finite sets as being subject to arbitrary permutation automorphisms. That is to say, we think of the set $\mathbb{S}_{j+1}$ as a container for the labels $0,1, \ldots, j$. Thus, for each $j$, one need only contend with an indeterminacy of $j+1$ possibilities for the element of this container that corresponds to $j$. In particular, if one allows $j=0, \ldots, l^{*}$ to vary, then this approach allows one to reduce the resulting label indeterminacy from a total of $\left(l^{ \pm}\right)^{l^{ \pm}}$possibilities [where we write $l^{ \pm}=1+l^{*}=$ $(l+1) / 2$ ] to a total of $l^{ \pm}$! [i.e., $\approx \sqrt{\left(l^{ \pm}\right)^{l^{ \pm}}}$] possibilities. It turns out that this reduction will yield just the right estimates in the log-volume computations to be performed in [IUTchIV]. Moreover, this approach satisfies the important property of insulating the "core label 0 " from the various label indeterminacies that occur.

Each element of each of the containers $\mathbb{S}_{j+1}$ may be thought of as parametrizing an $\mathcal{F}$ - or $\mathcal{D}$-prime-strip that occurs in the Hodge-Arakelov-theoretic evaluation of [IUTchII]. In order to render the construction multiradial, it is necessary to replace such holomorphic $\mathcal{F}$-/D -prime-strips by mono-analytic $\mathcal{F}^{\vdash}-/ \mathcal{D}^{\vdash}$-prime-strips. In particular, as discussed above, one may construct, for each such $\mathcal{F}^{\vdash}-/ \mathcal{D}^{\vdash}$-primestrip, a collection of log-shells associated to the various $\underline{v} \in \underline{\mathbb{V}}$. Write $\mathbb{V}_{\mathbb{Q}}$ for 
the set of valuations of $\mathbb{Q}$. Then, in order to obtain objects that are immune to the various label indeterminacies discussed above, we consider, for each element $* \in \mathbb{S}_{j+1}$, and for each [say, for simplicity, nonarchimedean] $v_{\mathbb{Q}} \in \mathbb{V}_{\mathbb{Q}}$,

- the direct sum of the log-shells associated to the prime-strip labeled by the given element $* \in \mathbb{S}_{j+1}$ at the $\underline{v} \in \underline{\mathbb{V}}$ that lie over $v_{\mathbb{Q}}$;

we then form

- the tensor product, over the elements $* \in \mathbb{S}_{j+1}$, of these direct sums.

This collection of tensor products associated to $v_{\mathbb{Q}} \in \mathbb{V}_{\mathbb{Q}}$ will be referred to as the tensor packet associated to the collection of prime-strips indexed by elements of $\mathbb{S}_{j+1}$. One may carry out this construction of the tensor packet either for holomorphic $\mathcal{F}$-/D -prime-strips [cf. Proposition 3.1] or for mono-analytic $\mathcal{F}^{\vdash}$-/ $\mathcal{D}^{\vdash}$-primestrips [cf. Proposition 3.2].

The tensor packets associated to $\mathcal{D}^{\vdash}$-prime-strips will play a crucial role in the theory of $\S 3$, as "multiradial mono-analytic containers" for the principal objects of interest [cf. the discussion of Remark 3.12.2, (ii)], namely,

- the action of the splitting monoids of the LGP-monoids - i.e., the monoids generated by the theta values $\left\{\underline{\underline{q}}_{\underline{v}}^{j^{2}}\right\}_{j=1, \ldots, l *}$ - on the portion of the tensor packets just defined at $\underline{v} \in \underline{\mathbb{V}}^{\text {bad }}$ [cf. Fig. I.4 below; Propositions 3.4, 3.5; the discussion of [IUTchII], Introduction];

- the action of copies " $\left(F_{\text {mod }}^{\times}\right)_{j}$ " of [the multiplicative monoid of nonzero elements of] the number field $F_{\text {mod }}$ labeled by $j=1, \ldots, l^{*}$ on the product, over $v_{\mathbb{Q}} \in \mathbb{V}_{\mathbb{Q}}$, of the portion of the tensor packets just defined at $v_{\mathbb{Q}}$ [cf. Fig. I.5 below; Propositions 3.3, 3.7, 3.10].

$$
\begin{aligned}
& \underline{\underline{q}}^{1} \curvearrowright \quad \underline{\underline{q}}^{j^{2}} \curvearrowright \quad \underline{\underline{q}}^{\left(l^{*}\right)^{2}} \curvearrowright \\
& /^{ \pm} \hookrightarrow /^{ \pm} / \pm \hookrightarrow \ldots \hookrightarrow /^{ \pm} /^{ \pm} \ldots /^{ \pm} \hookrightarrow{ }^{ \pm} \ldots \hookrightarrow /^{ \pm} / \pm \ldots \ldots /^{ \pm} \\
& \mathbb{S}_{1}^{ \pm} \quad \mathbb{S}_{1+1=2}^{ \pm} \quad \mathbb{S}_{j+1}^{ \pm} \quad \mathbb{S}_{1+l^{*}=l^{ \pm}}^{ \pm}
\end{aligned}
$$

Fig. I.4: Splitting monoids of LGP-monoids acting on tensor packets

$$
\begin{aligned}
& \left(F_{\text {mod }}^{\times}\right)_{1} \curvearrowright \quad\left(F_{\text {mod }}^{\times}\right)_{j} \curvearrowright \quad\left(F_{\text {mod }}^{\times}\right)_{l *} \curvearrowright \\
& /^{ \pm} \hookrightarrow /^{ \pm} /^{ \pm} \hookrightarrow \ldots \hookrightarrow /^{ \pm} / \pm \ldots /^{ \pm} \hookrightarrow{ }^{ \pm} \ldots \hookrightarrow /^{ \pm} / \pm \ldots \ldots /^{ \pm} \\
& \mathbb{S}_{1}^{ \pm} \quad \mathbb{S}_{1+1=2}^{ \pm} \quad \mathbb{S}_{j+1}^{ \pm} \quad \mathbb{S}_{1+l^{*}=l^{ \pm}}^{ \pm}
\end{aligned}
$$

Fig. I.5: Copies of $F_{\text {mod }}^{\times}$acting on tensor packets 
Indeed, these [splitting monoids of] LGP-monoids and copies " $\left(F_{\text {mod }}^{\times}\right)_{j}$ " of [the multiplicative monoid of nonzero elements of] the number field $F_{\bmod }$ admit natural embeddings into/actions on the various tensor packets associated to labeled $\mathcal{F}$ prime-strips in each $\Theta^{ \pm \text {ell }} \mathrm{NF}$-Hodge theater ${ }^{n, m} \mathcal{H} \mathcal{T}^{\Theta^{ \pm \mathrm{ell}} \mathrm{NF}}$ of the log-theta-lattice. One then obtains vertically coric versions of these splitting monoids of LGPmonoids and labeled copies " $\left(F_{\mathrm{mod}}^{\times}\right)_{j}$ " of [the multiplicative monoid of nonzero elements of] the number field $F_{\text {mod }}$ by applying appropriate Kummer isomorphisms between

- log-shells/tensor packets associated to [labeled] $\mathcal{F}$-prime-strips and

- log-shells/tensor packets associated to [labeled] D-prime-strips.

Finally, by passing to the

- log-shells/tensor packets associated to [labeled] $\mathcal{D}^{\vdash}$-prime-strips

- i.e., by forgetting the arithmetic holomorphic structure associated to a specific vertical line of the log-theta-lattice - one obtains the desired multiradial representation, i.e., description in terms that make sense from the point of view of an alien arithmetic holomorphic structure, of the splitting monoids of LGPmonoids and labeled copies of the number field $F_{\bmod }$ discussed above. This passage to the multiradial representation is obtained by admitting the following three types of indeterminacy:

(Ind1): This is the indeterminacy that arises from the automorphisms of processions of $\mathcal{D}^{\vdash}$-prime-strips that appear in the multiradial representation - i.e., more concretely, from permutation automorphisms of the label sets $\mathbb{S}_{j+1}$ that appear in the processions discussed above, as well as from the automorphisms of the $\mathcal{D}^{\vdash}$-prime-strips that appear in these processions.

(Ind2): This is the indeterminacy that arises from the automorphisms of the

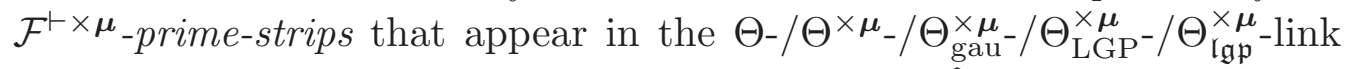
- i.e., in particular, at [for simplicity] $\underline{v} \in \underline{\mathbb{V}}^{\text {non }}$, the $\widehat{\mathbb{Z}}^{\times}$-indeterminacies acting on local copies of " $\mathcal{O}^{\times \mu}$ " [cf. the above discussion].

(Ind3): This is the indeterminacy that arises from the upper semi-compatibility of the log-Kummer correspondences associated to the specific vertical line of the log-theta-lattice under consideration [cf. the above discussion].

A detailed description of this multiradial representation, together with the indeterminacies (Ind1), (Ind2) is given in Theorem 3.11, (i) [and summarized in Theorem A, (i), below; cf. also Fig. I.6 below].

One important property of the multiradial representation discussed above concerns the relationship between the three main components - i.e., roughly speaking, log-shells, splitting monoids of LGP-monoids, and number fields - of this multiradial representation and the log-Kummer correspondence of the specific vertical line of the log-theta-lattice under consideration. This property — which may be thought of as a sort of "non-interference", or "mutual compatibility", property - asserts that the multiplicative monoids constituted by the splitting monoids 
of LGP-monoids and copies of $F_{\text {mod }}^{\times}$"do not interfere", relative to the various arrows that occur in the log-Kummer correspondence, with the local integers at $\underline{v} \in \underline{\mathbb{V}}$, hence, in particular, with the local units at $\underline{v} \in \underline{\mathbb{V}}$, that give rise to the log-shells. In the case of splitting monoids of LGP-monoids, this non-interference/mutual compatibility property is, in essence, a formal consequence of the existence of the canonical splittings [up to roots of unity] discussed in [IUTchII], Introduction, of the theta/Gaussian monoids that appear into unit group and value group portions. Here, we recall that, in the case of the theta monoids, these canonical splittings are, in essence, a formal consequence of the constant multiple rigidity property of mono-theta environments reviewed above. In the case of copies of $F_{\text {mod }}$, this non-interference/mutual compatibility property is, in essence, a formal consequence of the well-known fact in elementary algebraic number theory that any nonzero element of a number field that is integral at every valuation of the number field is necessarily a root of unity. These mutual compatibility properties are described in detail in Theorem 3.11, (ii), and summarized in Theorem A, (ii), below.

Another important property of the multiradial representation discussed above concerns the relationship between the three main components - i.e., roughly speaking, log-shells, splitting monoids of LGP-monoids, and number fields - of this multiradial representation and the $\Theta_{\mathrm{LGP}}^{\times \boldsymbol{\mu}}$-links, i.e., the horizontal arrows of the log-theta-lattice under consideration. This property - which may be thought of as a property of compatibility with the $\Theta_{\text {LGP }}^{\times \boldsymbol{\mu}}$-link - asserts that the cyclotomic rigidity isomorphisms that appear in the Kummer theory concerning the splitting monoids of LGP-monoids and copies of $F_{\text {mod }}^{\times}$are immune to the $\widehat{\mathbb{Z}}^{\times}$-indeterminacies

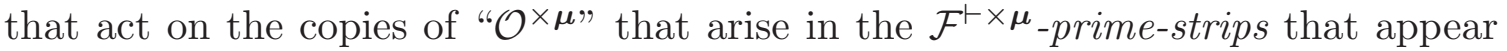
in the $\Theta_{\mathrm{LGP}}^{\times \boldsymbol{\mu}}$-link. In the case of splitting monoids of LGP-monoids, this property amounts precisely to the multiradiality theory developed in $\S 2$ [cf. the above discussion], i.e., in essence, to the mono-theta-theoretic cyclotomic rigidity property reviewed in the above discussion. In the case of copies of $F_{\text {mod }}^{\times}$, this property follows from the theory surrounding the construction of the cyclotomic rigidity isomorphism discussed in [IUTchI], Example 5.1, (v), together with the well-known fact in elementary algebraic number theory that any nonzero element of a number field that is integral at every valuation of the number field is necessarily a root of unity. These compatibility properties are described in detail in Theorem 3.11, (iii), and summarized in Theorem A, (iii), below.

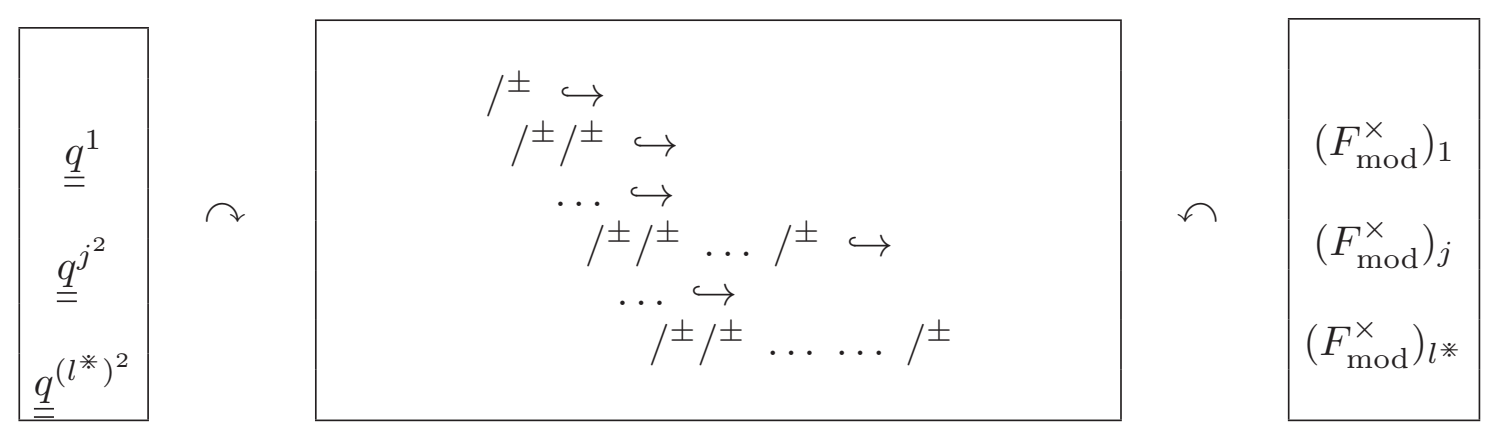

Fig. I.6: The full multiradial representation

At this point, we pause to observe that although considerable attention has been devoted so far in the present series of papers, especially in [IUTchII], to 
the theory of Gaussian monoids, not so much attention has been devoted [i.e., outside of [IUTchI], §5; [IUTchII], Corollaries 4.7, 4.8] to [the multiplicative monoids constituted by] copies of $F_{\text {mod }}^{\times}$. These copies of $F_{\text {mod }}^{\times}$enter into the theory of the multiradial representation discussed above in the form of various types of global Frobenioids in the following way. If one starts from the number field $F_{\text {mod }}$, one natural Frobenioid that can be associated to $F_{\text {mod }}$ is the Frobenioid $\mathcal{F}_{\text {mod }}^{\odot}$ of [stacktheoretic] arithmetic line bundles on [the spectrum of the ring of integers of] $F_{\bmod }$ discussed in [IUTchI], Example 5.1, (iii) [cf. also Example 3.6 of the present paper]. From the point of view of the theory surrounding the multiradial representation discussed above, there are two natural ways to approach the construction of " $\mathcal{F}_{\bmod }^{\odot}$ ":

(๑MOD) (Rational Function Torsor Version): This approach consists of considering the category $\mathcal{F}_{\text {MOD }}^{\odot}$ of $F_{\text {mod }}^{\times}$-torsors equipped with trivializations at each $\underline{v} \in \underline{\mathbb{V}}$ [cf. Example 3.6, (i), for more details].

$\left(\odot_{\mathfrak{m} \mathfrak{d}}\right)$ (Local Fractional Ideal Version): This approach consists of considering the category $\mathcal{F}_{\mathfrak{m o d}}^{\odot}$ of collections of integral structures on the various completions $K_{\underline{v}}$ at $\underline{v} \in \underline{\mathbb{V}}$ and morphisms between such collections of integral structures that arise from multiplication by elements of $F_{\bmod }^{\times}[\mathrm{cf}$. Example 3.6, (ii), for more details].

Then one has natural isomorphisms of Frobenioids

$$
\mathcal{F}_{\bmod }^{\odot} \stackrel{\sim}{\rightarrow} \mathcal{F}_{\mathrm{MOD}}^{\odot} \stackrel{\sim}{\rightarrow} \mathcal{F}_{\text {mod }}^{\odot}
$$

that induce the respective identity morphisms $F_{\mathrm{mod}}^{\times} \rightarrow F_{\mathrm{mod}}^{\times} \rightarrow F_{\mathrm{mod}}^{\times}$on the associated rational function monoids [cf. [FrdI], Corollary 4.10]. In particular, at first glance, $\mathcal{F}_{\text {MOD }}^{\odot}$ and $\mathcal{F}_{\text {mod }}^{\odot}$ appear to be "essentially equivalent" objects.

On the other hand, when regarded from the point of view of the multiradial representations discussed above, these two constructions exhibit a number of significant differences - cf. Fig. I.7 below; the discussion of Remarks 3.6.2, 3.10.2. For instance, whereas the construction of $\left(\bigcirc_{\mathrm{MOD}}\right)$ depends only on the multiplicative structure of $F_{\text {mod }}^{\times}$, the construction of $\left(\bigcirc_{\mathfrak{m o d}}\right)$ involves the module, i.e., the additive, structure of the localizations $K_{\underline{v}}$. The global portion of the $\Theta_{\mathrm{LGP}}^{\times \boldsymbol{\mu}}$-link (respectively, the $\Theta_{\mathfrak{l g p}}^{\times \boldsymbol{\mu}}$-link) is, by definition [cf. Definition 3.8, (ii)], constructed by means of the realification of the Frobenioid that appears in the construction of $\left(\odot_{\mathrm{MOD}}\right)$ (respectively, $\left.\left(\bigcirc_{\mathfrak{m o d}}\right)\right)$. This means that the construction of the global portion of the $\Theta_{\mathrm{LGP}}^{\times \boldsymbol{\mu}}-$ link — which is the version of the $\Theta$-link that is in fact ultimately used in the theory of the multiradial representation - depends only on the multiplicative monoid structure of a copy of $F_{\text {mod }}^{\times}$, together with the various valuation homomorphisms $F_{\bmod }^{\times} \rightarrow \mathbb{R}$ associated to $\underline{v} \in \underline{\mathbb{V}}$. Thus, the mutual compatibility [discussed above] of copies of $F_{\text {mod }}^{\times}$with the log-Kummer correspondence implies that one may perform this construction of the global portion of the $\Theta_{\mathrm{LGP}}^{\times \boldsymbol{\mu}}$-link in a fashion that is immune to the "upper semi-compatibility" indeterminacy (Ind3) [discussed above]. By contrast, the construction of $\left(\odot_{\mathfrak{m o d}}\right)$ involves integral structures on the underlying local additive modules " $K_{\underline{v}}$ ", i.e., from the point of view of the multiradial representation, integral structures on log-shells and tensor packets of log-shells, which are subject to the "upper semi-compatibility" indeterminacy 
(Ind3) [discussed above]. In particular, the log-Kummer correspondence subjects the construction of $\left(\odot_{\mathfrak{m o d}}\right)$ to "substantial distortion". On the other hand, the essential role played by local integral structures in the construction of $\left(\odot_{\mathfrak{m o d}}\right)$ enables one to compute the global arithmetic degree of the arithmetic line bundles constituted by objects of the category " $\mathcal{F}_{\mathfrak{m o d}}^{\odot}$ " in terms of log-volumes on log-shells and tensor packets of log-shells [cf. Proposition 3.9, (iii)]. This property of the construction of $\left(\odot_{\mathfrak{m o d}}\right)$ will play a crucial role in deriving the explicit estimates for such log-volumes that are obtained in Corollary 3.12 [cf. Theorem B below].

\begin{tabular}{|c|c|}
\hline $\mathcal{F}_{\mathrm{MOD}}^{\odot}$ & $\mathcal{F}_{\mathfrak{m o d}}^{\odot}$ \\
\hline $\begin{array}{c}\text { biased toward } \\
\text { multiplicative structures }\end{array}$ & $\begin{array}{l}\text { biased toward } \\
\text { additive structures }\end{array}$ \\
\hline $\begin{array}{c}\text { easily related to } \\
\text { value group/non-coric portion } \\
\text { "(-) }(-)^{\Vdash \prime} \text { of } \Theta_{\mathrm{LGP}}^{\times \boldsymbol{\mu}} \text {-link }\end{array}$ & $\begin{array}{l}\text { easily related to unit group/coric } \\
\text { portion " }(-)^{\vdash \times \mu " ~ o f ~} \Theta_{\mathrm{LGP}}^{\times \mu}-/ \Theta_{\mathfrak{l g} \mathfrak{p}}^{\times \boldsymbol{\mu}}-\text { link, } \\
\text { i.e., mono-analytic log-shells }\end{array}$ \\
\hline $\begin{array}{l}\text { admits } \\
\text { precise log-Kummer } \\
\text { correspondence }\end{array}$ & $\begin{array}{c}\text { only admits } \\
\text { "upper semi-compatible" } \\
\text { log-Kummer correspondence }\end{array}$ \\
\hline $\begin{array}{l}\text { rigid, but not suited } \\
\text { to explicit computation }\end{array}$ & $\begin{array}{l}\text { subject to substantial distortion, } \\
\text { but suited to explicit estimates }\end{array}$ \\
\hline
\end{tabular}

Fig. I.7: $\mathcal{F}_{\text {MOD }}^{\odot}$ versus $\mathcal{F}_{\text {mod }}^{\odot}$

Thus, in summary, the natural isomorphism $\mathcal{F}_{\text {MOD }}^{\odot} \stackrel{\sim}{\rightarrow} \mathcal{F}_{\text {mod }}^{\odot}$ discussed above plays the important role, in the context of the multiradial representation discussed above, of relating

- the multiplicative structure of the global number field $F_{\bmod }$ to the additive structure of $F_{\text {mod }}$;

- the unit group/coric portion " $(-)^{\vdash \times \mu}$ " of the $\Theta_{\mathrm{LGP}}^{\times \boldsymbol{\mu}}$-link to the value group/non-coric portion " $(-)^{\Vdash \Vdash}$ ” of the $\Theta_{\mathrm{LGP}}^{\times \mu}$-link.

Finally, in Corollary 3.12 [cf. also Theorem B below], we apply the multiradial representation discussed above to estimate certain log-volumes as follows. We begin by introducing some terminology [cf. Definition 3.8, (i)]. We shall refer to the object that arises in any of the versions [including realifications] of the global Frobenioid " $\mathcal{F}_{\bmod }^{\odot}$ " discussed above — such as, for instance, the realified global Frobenioid 
that occurs in the codomain of the $\Theta_{\mathrm{gau}^{\circ}}^{\times \boldsymbol{\mu}} / \Theta_{\mathrm{LGP}^{-}}^{\times \boldsymbol{\mu}} / \Theta_{\mathfrak{l g p}}^{\times \boldsymbol{\mu}}$-link - by considering the arithmetic divisor determined by the zero locus of the elements " $\underline{\underline{q}} v$ " at $\underline{v} \in \underline{\mathbb{V}}^{\text {bad }}$ as a $q$-pilot object. The log-volume of the $\boldsymbol{q}$-pilot object will be $\overline{\text { denoted by }}$

$$
-|\log (\underline{q})| \in \mathbb{R}
$$

— so $|\log (\underline{\underline{q}})|>0$ [cf. Corollary 3.12; Theorem B]. In a similar vein, we shall refer to the object that arises in the realified global Frobenioid that occurs in the domain of the $\Theta_{\mathrm{gau}^{-}}^{\times \boldsymbol{\mu}} / \Theta_{\mathrm{LGP}^{-}}^{\times \boldsymbol{\mu}} / \Theta_{\mathfrak{l g p}^{\circ}}^{\times \boldsymbol{\mu}}$-link by considering the arithmetic divisor determined by the zero locus of the collection of theta values " $\left\{\underline{\underline{q}}_{\underline{v}}^{j^{2}}\right\}_{j=1, \ldots, l *}$ " at $\underline{v} \in \underline{\mathbb{V}}^{\text {bad }}$ as a $\Theta$-pilot object. The log-volume of the union of the collection of possible images of the $\Theta$-pilot object in the multiradial representation - i.e., where we recall that these "possible images" are subject to the indeterminacies (Ind1), (Ind2), (Ind3) — will be denoted by

$$
-|\log (\underline{\underline{\Theta}})| \in \mathbb{R} \bigcup\{+\infty\}
$$

[cf. Corollary 3.12; Theorem B]. Then the content of Corollary 3.12, Theorem B may be summarized, roughly speaking [cf. Remark 3.12.1, (ii)], as a result concerning the

$$
\text { negativity of the } \Theta \text {-pilot log-volume }|\log (\underline{\underline{\Theta}})| \text {. }
$$

Relative to the analogy between the theory of the present series of papers and complex/p-adic Teichmüller theory [cf. [IUTchI], §I4], this result may be thought of as a statement to the effect that

"the pair consisting of a number field equipped with an elliptic curve is metrically hyperbolic, i.e., has negative curvature".

That is to say, it may be thought of as a sort of analogue of the inequality

$$
\chi_{S}=-\int_{S} d \mu_{S}<0
$$

arising from the classical Gauss-Bonnet formula on a hyperbolic Riemann surface of finite type $S$ [where we write $\chi_{S}$ for the Euler characteristic of $S$ and $d \mu_{S}$ for the Kähler metric on $S$ determined by the Poincaré metric on the upper half-plane — cf. the discussion of Remark 3.12.3], or, alternatively, of the inequality

$$
(1-p)\left(2 g_{X}-2\right) \leq 0
$$

that arises by computing global degrees of line bundles in the context of the Hasse invariant that arises in $p$-adic Teichmüller theory [where $X$ is a smooth, proper hyperbolic curve of genus $g_{X}$ over the ring of Witt vectors of a perfect field of characteristic $p$ which is canonical in the sense of $p$-adic Teichmüller theory - cf. the discussion of Remark 3.12.4, (v)].

The proof of Corollary 3.12 [i.e., Theorem B] is based on the following fundamental observation: the multiradial representation discussed above yields 
two tautologically equivalent ways to compute the $\boldsymbol{q}$-pilot log-volume $-|\log (\underline{\underline{q}})|$

- cf. Fig. I.8 below; Step (xi) of the proof of Corollary 3.12. That is to say, suppose that one starts with the $\boldsymbol{q}$-pilot object in the $\Theta^{ \pm \text {ell }}$ NF-Hodge theater ${ }^{1,0} \mathcal{H} \mathcal{T}^{\Theta^{ \pm e l 1} \mathrm{NF}}$ at $(1,0)$, which we think of as being represented, via the approach of $\left(\odot_{\mathfrak{m} \mathfrak{o}}\right)$, by means of the action of the various $\underline{\underline{q}}_{\underline{v}}$, for $\underline{v} \in \underline{\mathbb{V}}^{\text {bad }}$, on the logshells that arise, via the log-link ${ }^{1,-1} \mathcal{H} \mathcal{T}^{\Theta^{ \pm \text {ell }} \mathrm{NF}} \stackrel{-\mathfrak{l o g}}{\longrightarrow} 1,0 \mathcal{H} \mathcal{T}^{\Theta^{ \pm e l l}} \mathrm{NF}$, from the various local " $\mathrm{O}^{\times \boldsymbol{\mu}}$ 's" in the $\Theta^{ \pm \text {ell }} \mathrm{NF}$-Hodge theater ${ }^{1,-1} \mathcal{H}^{\Theta^{ \pm \text {ell }} \mathrm{NF}}$ at $(1,-1)$.

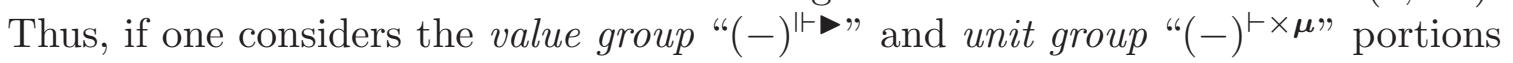
of the codomain of the $\Theta_{\mathrm{LGP}}^{\times \mu}-\operatorname{link}{ }^{0,0} \mathcal{H} \mathcal{T}^{\Theta^{ \pm \mathrm{ell}} \mathrm{NF}} \stackrel{\Theta_{\mathrm{LGP}}^{\times \mu}}{\longrightarrow} 1,0 \mathcal{H} \mathcal{T}^{\Theta^{ \pm \mathrm{ell}} \mathrm{NF}}$ in the context of the arithmetic holomorphic structure of the vertical line $(1, \circ)$, this action on log-shells may be thought of as a somewhat intricate "intertwining" between these value group and unit group portions. On the other hand, the $\Theta_{\mathrm{LGP}}^{\times \boldsymbol{\mu}}$-link ${ }^{0,0} \mathcal{H} \mathcal{T}^{\Theta^{ \pm e l l} \mathrm{NF}} \stackrel{\Theta_{\mathrm{LGP}}^{\times \mu}}{\longrightarrow} 1,0 \mathcal{H} \mathcal{T}^{\Theta^{ \pm e l l} \mathrm{NF}}$ constitutes a sort of gluing isomorphism between the arithmetic holomorphic structures associated to the vertical lines $(0,0)$ and $(1, \circ)$ that is based on

forgetting this intricate intertwining, i.e., by working solely with abstract isomorphisms of $\mathcal{F}^{\Vdash \triangleright \times \boldsymbol{\mu}}$-prime-strips.

Thus, in order to relate the arithmetic holomorphic structures, say, at $(0,0)$ and $(1,0)$, one must apply the multiradial representation discussed above. That is to say, one starts by applying the theory of bi-coric mono-analytic log-shells given in Theorem 1.5. One then applies the Kummer theory surrounding the splitting monoids of theta/Gaussian monoids and copies of the number field $F_{\text {mod, }}$ which allows one to pass from the Frobenius-like versions of various objects that appear in - i.e., that are necessary in order to consider - the $\Theta_{\mathrm{LGP}}^{\times \mu}$-link to the corresponding étale-like versions of these objects that appear in the multiradial representation. This passage from Frobenius-like versions to étale-like versions is referred to as the operation of Kummer-detachment [cf. Fig. I.8; Remark 1.5.4, (i)]. As discussed above, this operation of Kummer-detachment is possible precisely as a consequence of the compatibility of the multiradial representation with the $\Theta_{\mathrm{LGP}}^{\times \boldsymbol{\mu}}$-link, i.e., with the indeterminacy (Ind2). Moreover, since the logtheta-lattice is, as discussed above, far from commutative, in order to represent the various "log-link-conjugates" at $(0, m)[$ for $m \in \mathbb{Z}]$ in terms that may be understood from the point of view of the arithmetic holomorphic structure at $(1,0)$, one must work [not only with the Kummer isomorphisms at a single $(0, m)$, but rather with] the entire log-Kummer correspondence. In particular, one must take into account the indeterminacy (Ind3). Once one completes the operation of Kummer-detachment so as to obtain vertically coric versions of objects on the vertical line $(0, \circ)$, one then passes to multiradial objects, i.e., to the "final form" of the multiradial representation, by introducing the indeterminacy (Ind1), i.e., that arises from working with [mono-analytic!] $\mathcal{D}^{\vdash}$ - [as opposed to $\mathcal{D}$-!] prime-strips. Finally, one computes the log-volume of this "final form" multiradial representation of the $\Theta$-pilot object - i.e., subject to the indeterminacies (Ind1), (Ind2), (Ind3)! — and concludes the desired estimates from the tautological observation that 
the log-theta-lattice, and, in particular, the "gluing isomorphism" constituted by the $\Theta_{\mathrm{LGP}}^{\times \boldsymbol{\mu}}-\mathbf{l i n k}$, were constructed precisely in such a way as to assure that this computation of $|\log (\underline{\Theta})|$, i.e., of possible log-volumes of the $\Theta$-pilot object, necessarily contains $|\log (\underline{\underline{q}})|$ [i.e., as a "possible log-volume of the $\Theta$-pilot object"]

— cf. Fig. I.8 below; Step (xi) of the proof of Corollary 3.12. That is to say, the "gluing isomorphism" constituted by the $\Theta_{\mathrm{LGP}}^{\times \mu}$-link relates two distinct "arithmetic holomorphic structures", i.e., two distinct copies of conventional ring/scheme theory, that are glued together precisely by means of a relation that identifies the $\Theta$-pilot object in the domain of the $\Theta_{\mathrm{LGP}}^{\times \boldsymbol{\mu}}$-link with the $q$-pilot object in the codomain of the $\Theta_{\mathrm{LGP}}^{\times \mu}-$ link. Thus, once one sets up such an apparatus, the computation of the log-volume of the $\Theta$-pilot object in the domain of the $\Theta_{\mathrm{LGP}}^{\times \mu}$-link in terms of the $q$-pilot object in the codomain of the $\Theta_{\mathrm{LGP}}^{\times \mu}$-link amounts - tautologically! to the computation of the log-volume of the $q$-pilot object [in the codomain of the $\Theta_{\mathrm{LGP}}^{\times \boldsymbol{\mu}}$-link] in terms of itself, i.e., to a computation that reflects certain intrinsic properties of this $q$-pilot object. This is the content of Corollary 3.12 [i.e., Theorem B]. As discussed above, this sort of "computation of intrinsic properties" in the present context of a number field equipped with an elliptic curve may be regarded as analogous to the "computations of intrinsic properties" reviewed above in the classical complex and $p$-adic cases.
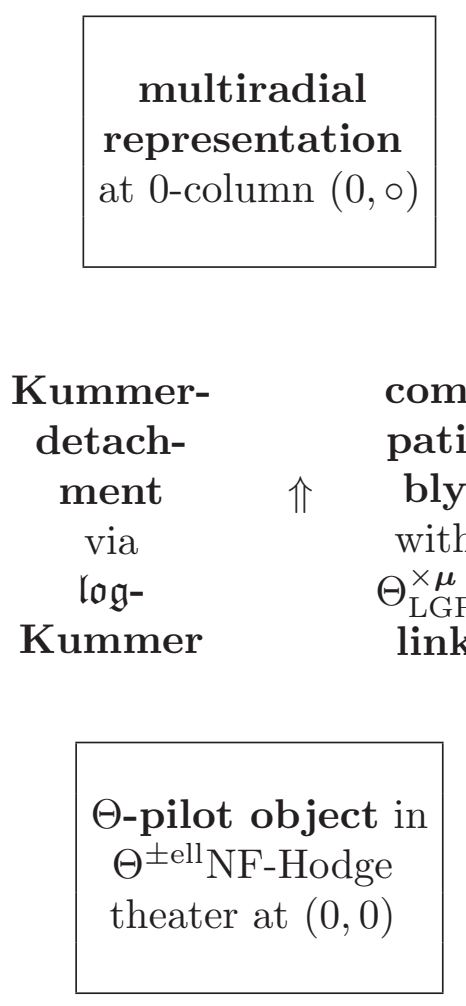

$$
\begin{aligned}
& \text { permutation } \\
& \text { symmetry of } \\
& \approx \\
& \text { étale-picture }
\end{aligned}
$$

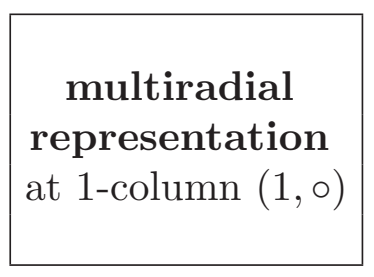

Fig. I.8: Two tautologically equivalent ways to compute the log-volume of the $q$-pilot object at $(1,0)$

We conclude the present Introduction with the following summaries of the main results of the present paper. 
Theorem A. (Multiradial Algorithms for Logarithmic Gaussian Procession Monoids) Fix a collection of initial $\Theta$-data $\left(\bar{F} / F, X_{F}, l, \underline{C}_{K}, \underline{\mathbb{V}}, \underline{\epsilon}\right)$ as in [IUTchI], Definition 3.1. Let

$$
\left\{{ }^{n, m} \mathcal{H} \mathcal{T}^{\Theta^{ \pm \mathrm{ell}} \mathrm{NF}}\right\}_{n, m \in \mathbb{Z}}
$$

be a collection of distinct $\Theta^{ \pm \text {ell }} \mathbf{N F - H o d g e ~ t h e a t e r s ~ [ r e l a t i v e ~ t o ~ t h e ~ g i v e n ~ i n i t i a l ~}$ $\Theta$-data] - which we think of as arising from a LGP-Gaussian log-theta-lattice [cf. Definition 3.8, (iii)]. For each $n \in \mathbb{Z}$, write

$$
{ }^{n,{ }^{\circ}} \mathcal{H} \mathcal{T}^{\mathcal{D}-\Theta^{ \pm \mathrm{ell}} \mathrm{NF}}
$$

for the $\mathcal{D}-\Theta^{ \pm \text {ell }} N F$-Hodge theater determined, up to isomorphism, by the various $n, m \mathcal{H} \mathcal{T}^{\Theta^{ \pm e l l} \mathrm{NF}}$, where $m \in \mathbb{Z}$, via the vertical coricity of Theorem 1.5, (i) [cf. Remark 3.8.2].

(i) (Multiradial Representation) Write

$$
{ }^{n,{ }^{\circ}} \mathfrak{R}^{\mathrm{LGP}}
$$

for the collection of data consisting of

(a) tensor packets of log-shells;

(b) splitting monoids of LGP-monoids acting on the tensor packets of (a);

(c) copies, labeled by $j \in \mathbb{F}_{i}^{*}$, of [the multiplicative monoid of nonzero elements of] the number field $F_{\bmod }$ acting on the tensor packets of (a)

[cf. Theorem 3.11, (i), (a), (b), (c), for more details] regarded up to indeterminacies of the following two types:

(Ind1) the indetermacies induced by the automorphisms of the procession of $\mathcal{D}^{\vdash}$-prime-strips $\operatorname{Prc}\left({ }^{n, \circ} \mathfrak{D}_{T}^{\vdash}\right)$ that gives rise to the tensor packets of (a);

(Ind2) the indeterminacies that arise from the automorphisms of the $\mathcal{F}^{+\times \boldsymbol{\mu}_{-}}$ prime-strips that appear in the $\Theta_{\mathrm{LGP}}^{\times \boldsymbol{\mu}}-$ link, i.e., in particular, at [for simplicity] $\underline{v} \in \underline{\mathbb{V}}^{\text {non }}$, the $\widehat{\mathbb{Z}}^{\times}$-indeterminacies acting on local copies of "Oㅅ $"$

- cf. Theorem 3.11, (i), for more details. Then ${ }^{n, \circ} \mathfrak{R}^{\mathrm{LGP}}$ may be constructed via an algorithm in the procession of $\mathcal{D}^{\vdash}$-prime-strips $\operatorname{Prc}\left({ }^{n, \circ} \mathfrak{D}_{T}^{\vdash}\right)$, which is functorial with respect to isomorphisms of processions of $\mathcal{D}^{-}$-prime-strips. For $n, n^{\prime} \in \mathbb{Z}$, the permutation symmetries of the étale-picture discussed in [IUTchI], Corollary 6.10, (iii); [IUTchII], Corollary 4.11, (ii), (iii) [cf. also Corollary 2.3, (ii); Remark 3.8.2, of the present paper], induce compatible poly-isomorphisms

$$
\operatorname{Prc}\left({ }^{n, \circ} \mathfrak{D}_{T}^{\vdash}\right) \stackrel{\sim}{\rightarrow} \operatorname{Prc}\left(n^{n^{\prime}, \circ} \mathfrak{D}_{T}^{\vdash}\right) ; \quad{ }^{n, \circ} \mathfrak{R}^{\mathrm{LGP}} \stackrel{\sim}{\rightarrow}{ }^{n^{\prime}, \circ} \mathfrak{R}^{\mathrm{LGP}}
$$


which are, moreover, compatible with the bi-coricity poly-isomorphisms

$$
{ }^{n, \circ} \mathcal{D}_{0}^{\vdash} \stackrel{\sim}{\rightarrow} n^{\prime},{ }^{\circ} \mathcal{D}_{0}^{\vdash}
$$

of Theorem 1.5, (iii) [cf. also [IUTchII], Corollaries 4.10, (iv); 4.11, (i)].

(ii) (log-Kummer Correspondence) For $n, m \in \mathbb{Z}$, the inverses of the Kummer isomorphisms associated to the various $\mathcal{F}$-prime-strips and NFbridges that appear in the $\Theta^{ \pm \mathrm{ell}} \mathrm{NF}$-Hodge theater ${ }^{n, m} \mathcal{H} \mathcal{T}^{\Theta^{ \pm \mathrm{ell}} \mathrm{NF}}$ induce "inverse Kummer" isomorphisms between the vertically coric data (a), (b), (c) of (i) and the corresponding Frobenioid-theoretic data arising from each $\Theta^{ \pm \text {ell }} \mathrm{NF}$ Hodge theater ${ }^{n, m} \mathcal{H} \mathcal{T}^{\Theta^{ \pm \mathrm{ell}} \mathrm{NF}}$ [cf. Theorem 3.11, (ii), (a), (b), (c), for more details]. Moreover, as one varies $m \in \mathbb{Z}$, the corresponding Kummer isomorphisms [i.e., inverses of "inverse Kummer" isomorphisms] of splitting monoids of LGP-monoids [cf. (i), (b)] and labeled copies of the number field $F_{\bmod }$ [cf. (i), (c)] are mutually compatible, relative to the log-links of the $n$-th column of the LGP-Gaussian log-theta-lattice under consideration, in the sense that the only portions of the [Frobenioid-theoretic] domains of these Kummer isomorphisms that are related to one another via the log-links consist of roots of unity [multiplication by which corresponds, via the log-link, to an "addition by zero" indeterminacy, i.e., to no indeterminacy!] - cf. Proposition 3.5, (ii), (c); Proposition 3.10, (ii); Theorem 3.11, (ii), for more details. On the other hand, the Kummer isomorphisms of tensor packets of log-shells [cf. (i), (a)] are subject to a certain "indeterminacy" as follows:

(Ind3) as one varies $m \in \mathbb{Z}$, these Kummer isomorphisms of tensor packets of log-shells are "upper semi-compatible", relative to the log-links of the $n$-th column of the LGP-Gaussian log-theta-lattice under consideration, in a sense that involves certain natural inclusions " $\subseteq$ " at $v_{\mathbb{Q}} \in \mathbb{V}_{\mathbb{Q}}^{\text {non }}$ and certain natural surjections " $\rightarrow$ " at $v_{\mathbb{Q}} \in \mathbb{V}_{\mathbb{Q}}^{\operatorname{arc}}-c f$. Proposition 3.5, (ii), (a), (b); Theorem 3.11, (ii), for more details.

Finally, as one varies $m \in \mathbb{Z}$, these Kummer isomorphisms of tensor packets of log-shells are [precisely!] compatible, relative to the log-links of the $n$-th column of the LGP-Gaussian log-theta-lattice under consideration, with the respective logvolumes [cf. Proposition 3.9, (iv)].

(iii) $\left(\Theta_{\mathrm{LGP}}^{\times \mu}\right.$-Link Compatibility) The various Kummer isomorphisms of (ii) satisfy compatibility properties with the various horizontal arrows - i.e., $\Theta_{\mathrm{LGP}^{-}}^{\times \boldsymbol{\mu}}$ links - of the LGP-Gaussian log-theta-lattice under consideration as follows: The tensor packets of log-shells [cf. (i), (a)] are compatible, relative to the relevant

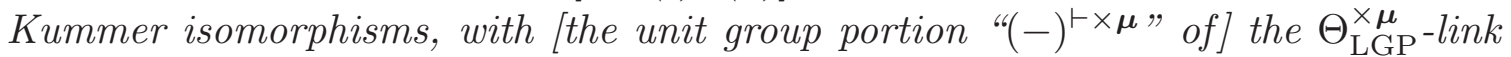
[cf. the indeterminacy "(Ind2)" of (i)]; we refer to Theorem 3.11, (iii), (a), (b), for more details. The identity automorphism on the objects that appear in the construction of the splitting monoids of LGP-monoids via mono-theta environments [cf. (i), (b)] is compatible, relative to the relevant Kummer isomorphisms and isomorphisms of mono-theta environments, with the $\Theta_{\mathrm{LGP}}^{\times \boldsymbol{\mu}}$-link [cf. the indeterminacy "(Ind2)" of (i)]; we refer to Theorem 3.11, (iii), (c), for more details. The identity automorphism on the objects that appear in the construction of the 
labeled copies of the number field $F_{\bmod }[c f .(i),(c)]$ is compatible, relative to the relevant Kummer isomorphisms and cyclotomic rigidity isomorphisms [cf. the discussion of Remark 3.10.1, (ii); the construction of [IUTchI], Example 5.1, (v)], with the $\Theta_{\mathrm{LGP}}^{\times \boldsymbol{\mu}}$-link [cf. the indeterminacy "(Ind2)" of (i)]; we refer to Theorem 3.11, (iii), (d), for more details.

Theorem B. (Log-volume Estimates for Multiradially Represented Splitting Monoids of Logarithmic Gaussian Procession Monoids) Suppose that we are in the situation of Theorem A. Write

$$
-|\log (\underline{\underline{\Theta}})| \in \mathbb{R} \bigcup\{+\infty\}
$$

for the procession-normalized mono-analytic log-volume [where the average is taken over $j \in \mathbb{F}_{l}^{*}$ - cf. Remark 3.1.1, (ii); Proposition 3.9, (i), (ii); Theorem 3.11, (i), (a), for more details] of the union of the possible images of a $\Theta$-pilot object [cf. Definition 3.8, (i)], relative to the relevant Kummer isomorphisms [cf. Theorems A, (ii); 3.11, (ii)], in the multiradial representation of Theorems $A$, (i); 3.11, (i), which we regard as subject to the indeterminacies (Ind1), (Ind2), (Ind3) described in Theorems A, (i), (ii); 3.11, (i), (ii). Write

$$
-|\log (\underline{\underline{q}})| \in \mathbb{R}
$$

for the procession-normalized mono-analytic log-volume of the image of a $\boldsymbol{q}$-pilot object [cf. Definition 3.8, (i)], relative to the relevant $\mathbf{K u m m e r}$ isomorphisms [cf. Theorems A, (ii); 3.11, (ii)], in the multiradial representation of Theorems A, (i); 3.11, (i), which we do not regard as subject to the indeterminacies (Ind1), (Ind2), (Ind3) described in Theorems A, (i), (ii); 3.11, (i), (ii). Here, we recall the definition of the symbol " $\triangle$ " as the result of identifying the labels

$$
\text { " } 0 \text { " and " }\left\langle\mathbb{F}_{i}^{*}\right\rangle "
$$

[cf. [IUTchII], Corollary 4.10, (i)]. In particular, $|\log (\underline{\underline{q}})|>0$ is easily computed in terms of the various $\boldsymbol{q}$-parameters of the elliptic curve $E_{F}$ [cf. [IUTchI], Definition 3.1, (b)] at $\underline{v} \in \underline{\mathbb{V}}^{\text {bad }}(\neq \emptyset)$. Then it holds that

$$
C_{\Theta} \geq-1
$$

for any real number $C_{\Theta} \in \mathbb{R}$ such that

$$
-|\log (\underline{\underline{\Theta}})| \leq C_{\Theta} \cdot|\log (\underline{\underline{q}})|
$$

[i.e., $-|\log (\underline{\underline{\Theta}})| \in \mathbb{R} \subseteq \mathbb{R} \bigcup\{+\infty\}$ and satisfies the inequality $\left.\left({ } C_{\Theta}\right)\right]$.

\section{Acknowledgements:}

I would like to thank Fumiharu Kato and Akio Tamagawa for many helpful discussions concerning the material presented in this paper.

\section{Notations and Conventions:}

We shall continue to use the "Notations and Conventions" of [IUTchI], $\S 0$. 


\section{Section 1: The Log-theta-lattice}

In the present $\S 1$, we discuss various enhancements to the theory of log-shells, as developed in [AbsTopIII]. In particular, we develop the theory of the log-link [cf. Definition 1.1; Propositions 1.2, 1.3], which, together with the $\Theta^{\times \boldsymbol{\mu}}$ - and $\Theta_{\text {gau }}^{\times \boldsymbol{\mu}}$-links of [IUTchII], Corollary 4.10, (iii), leads naturally to the construction of the logtheta-lattice, an apparatus that is central to the theory of the present series of papers. We conclude the present $\S 1$ with a discussion of various coric structures associated to the log-theta-lattice [cf. Theorem 1.5].

In the following discussion, we assume that we have been given initial $\Theta$-data as in [IUTchI], Definition 3.1. We begin by reviewing various aspects of the theory of log-shells developed in [AbsTopIII].

Definition 1.1. Let

$$
{ }^{\dagger} \mathfrak{F}=\left\{{ }^{\dagger} \mathcal{F}_{\underline{v}}\right\}_{\underline{v} \in \underline{\mathbb{V}}}
$$

be an $\mathcal{F}$-prime-strip [relative to the given initial $\Theta$-data - cf. [IUTchI], Definition 5.2 , (i)]. Write

$$
{ }^{\dagger} \mathfrak{F}^{\vdash}=\left\{{ }^{\dagger} \mathcal{F}_{\underline{v}}^{\vdash}\right\}_{\underline{v} \in \underline{\mathbb{V}}} ; \quad{ }^{\dagger} \mathfrak{F}^{\vdash \times \mu}=\left\{{ }^{\dagger} \mathcal{F}_{\underline{v}}^{\vdash \times \mu}\right\}_{\underline{v} \in \underline{\mathbb{V}}}
$$

for the associated $\mathcal{F}^{\vdash}$-, $\mathcal{F}^{\vdash \times \boldsymbol{\mu}}$-prime-strips [cf. [IUTchI], Remark 5.2.1, (ii); [IUTchII], Definition 4.9, (vi), (vii)]. Recall the functorial algorithm of [IUTchII], Corollary 4.6, (i), in the $\mathcal{F}$-prime-strip ${ }^{\dagger} \mathfrak{F}$ for constructing the assignment $\left.\Psi_{\text {cns }}{ }^{\dagger} \mathfrak{F}\right)$ given by

$$
\begin{aligned}
& \underline{\mathbb{V}}^{\text {non }} \ni \underline{v} \mapsto \Psi_{\mathrm{cns}}\left({ }^{\dagger} \mathfrak{F}\right)_{\underline{v}} \stackrel{\text { def }}{=}\left\{G_{\underline{v}}\left({ }^{\dagger} \Pi_{\underline{v}}\right) \curvearrowright \Psi_{\dagger \mathcal{F}_{\underline{v}}}\right\} \\
& \underline{\mathbb{V}}^{\operatorname{arc}} \ni \underline{v} \mapsto \Psi_{\mathrm{cns}}\left({ }^{\dagger} \mathfrak{F}\right)_{\underline{v}} \stackrel{\text { def }}{=} \Psi_{\dagger \mathcal{F}_{\underline{v}}}
\end{aligned}
$$

- where the data in brackets " $\{-\}$ " is to be regarded as being well-defined only up to a ${ }^{\dagger} \Pi_{\underline{v}}$-conjugacy indeterminacy [cf. [IUTchII], Corollary 4.6, (i), for more details]. In the following, we shall write

$$
(-) \underline{\text { gp }} \stackrel{\text { def }}{=}(-)^{\mathrm{gp}} \bigcup\{0\}
$$

for the formal union with $\{0\}$ of the groupification $(-)^{g p}$ of a monoid "(-)". Thus, by setting the product of all elements of $(-) \underline{g p}$ with 0 to be equal to 0 , one obtains a natural monoid structure on $(-) \underline{g p}$.

(i) Let $\underline{v} \in \underline{\mathbb{V}}^{\text {non }}$. Write

$$
\left(\Psi_{\dagger \mathcal{F}_{\underline{v}}} \supseteq \Psi_{\dagger \mathcal{F}_{\underline{v}}}^{\times} \rightarrow\right) \quad \Psi_{\dagger \mathcal{F}_{\underline{v}}}^{\sim} \stackrel{\text { def }}{=}\left(\Psi_{\dagger \mathcal{F}_{\underline{v}}}^{\times}\right)^{\mathrm{pf}}
$$

for the perfection $\left(\Psi_{\dagger \mathcal{F}_{\underline{v}}}^{\times}\right)^{\text {pf }}$ of the submonoid of units $\Psi_{\dagger \mathcal{F}_{\underline{v}}}^{\times}$of $\Psi_{\dagger \mathcal{F}_{\underline{v}}}$. Now let us recall from the theory of [AbsTopIII] [cf. [AbsTopIII], Definition 3.1, (iv); [AbsTopIII], Proposition 3.2, (iii), (v)] that the natural, algorithmically constructible topological field structure on $\Psi_{\dagger_{\underline{\mathcal{F}_{\underline{v}}}}}^{\mathrm{gp}}$ allows one to define a $p_{\underline{\underline{v}}}$-adic logarithm on $\Psi_{\dagger \mathcal{F}_{\underline{v}}}^{\sim}$, 
which, in turn, yields a functorial algorithm in the Frobenioid ${ }^{\dagger} \mathcal{F}_{\underline{v}}$ for constructing a topological field structure on $\Psi_{\dagger \mathcal{F}_{\underline{v}}}^{\sim}$. Write

$$
\Psi_{\mathfrak{l o g}\left(\dagger \mathcal{F}_{\underline{v}}\right)} \subseteq \Psi_{\dagger \mathcal{F}_{\underline{v}}}^{\sim}
$$

for the resulting multiplicative monoid of nonzero integers. Here, we observe that the resulting diagram

$$
\Psi_{\dagger \mathcal{F}_{\underline{v}}} \supseteq \Psi_{\dagger \mathcal{F}_{\underline{v}}}^{\times} \rightarrow \Psi_{\dagger \mathcal{F}_{\underline{v}}}^{\sim}=\Psi \frac{\mathrm{gp}}{\mathfrak{l o g}\left(\dagger \mathcal{F}_{\underline{v}}\right)}
$$

is compatible with the various natural actions of ${ }^{\dagger} \Pi_{v} \rightarrow G_{v}\left({ }^{\dagger} \Pi_{v}\right)$ on each of the [four] " $\Psi$ 's" appearing in this diagram. The pair $\left\{{ }^{\dagger} \Pi_{\underline{v}} \curvearrowright \Psi_{\mathfrak{l o g}\left(\dagger \mathcal{F}_{v}\right)}\right\}$ now determines a Frobenioid

$$
\mathfrak{l o g}\left({ }^{\dagger} \mathcal{F}_{\underline{v}}\right)
$$

[cf. [AbsTopIII], Remark 3.1.1; [IUTchI], Remark 3.3.2] — which is, in fact, naturally isomorphic to the Frobenioid ${ }^{\dagger} \mathcal{F}_{\underline{v}}$, but which we wish to think of as being related to ${ }^{\dagger} \mathcal{F}_{\underline{v}}$ via the above diagram. We shall denote this diagram by means of the notation

$$
\dagger \mathcal{F}_{\underline{v}} \stackrel{\log }{\longrightarrow} \log \left({ }^{\dagger} \mathcal{F}_{\underline{v}}\right)
$$

and refer to this relationship between ${ }^{\dagger} \mathcal{F}_{\underline{v}}$ and $\log \left({ }^{\dagger} \mathcal{F}_{\underline{v}}\right)$ as the tautological loglink associated to ${ }^{\dagger} \mathcal{F}_{\underline{v}}$ [or, when $\dagger \mathfrak{F}$ is fixed, at $\left.\underline{v}\right]$. If $\log \left({ }^{\dagger} \mathcal{F}_{\underline{v}}\right) \stackrel{\sim}{\rightarrow} \ddagger \mathcal{F}_{\underline{v}}$ is any [poly-]isomorphism of Frobenioids, then we shall write

$$
\dagger \mathcal{F}_{\underline{v}} \stackrel{\mathfrak{l o g}}{\longrightarrow} \ddagger \mathcal{F}_{\underline{v}}
$$

for the diagram obtained by post-composing the tautological log-link associated to ${ }^{\dagger} \mathcal{F}_{\underline{v}}$ with the given [poly-]isomorphism $\mathfrak{l o g}\left({ }^{\dagger} \mathcal{F}_{\underline{v}}\right) \stackrel{\sim}{\rightarrow} \ddagger \mathcal{F}_{\underline{v}}$ and refer to this relationship between $\dagger^{\dagger} \mathcal{F}_{\underline{v}}$ and $\ddagger \mathcal{F}_{\underline{v}}$ as a log-link from ${ }^{\dagger} \mathcal{F}_{\underline{v}}$ to $\ddagger \mathcal{F}_{\underline{v}}$; when the given [poly-]isomorphism $\log \left({ }^{\dagger} \mathcal{F}_{\underline{v}}\right) \stackrel{\sim}{\rightarrow} \ddagger \mathcal{F}_{\underline{v}}$ is the full poly-isomorphism, then we shall refer to the resulting $\mathfrak{l o g}$-link as the full $\mathfrak{l o g}$-link from ${ }^{\dagger} \mathcal{F}_{\underline{v}}$ to ${ }^{\ddagger} \mathcal{F}_{\underline{v}}$. Finally, we recall from [AbsTopIII], Definition 3.1, (iv), that the image in $\Psi_{\uparrow_{\mathcal{F}_{\underline{v}}}^{\sim}}^{\sim}$ of the submonoid of $G_{\underline{v}}\left({ }^{\dagger} \Pi_{\underline{v}}\right)$-invariants of $\Psi_{\dagger \mathcal{F}_{\underline{v}}}^{\times}$constitutes a compact topological module, which we shall refer to as the pre-log-shell. Write $p_{\underline{v}}^{*} \stackrel{\text { def }}{=} p_{\underline{v}}$ when $p_{\underline{v}}$ is odd and $p_{\underline{v}}^{*} \stackrel{\text { def }}{=} p_{\underline{v}}^{2}$ when $p_{\underline{v}}$ is even. Then we shall refer to the result of multiplying the pre-log-shell by the factor $\left(p_{\underline{v}}^{*}\right)^{-1}$ as the log-shell

$$
\mathcal{I}_{\dagger \mathcal{F}_{\underline{v}}} \subseteq \Psi_{\dagger \mathcal{F}_{\underline{v}}}^{\sim}=\Psi \frac{\mathrm{gp}}{\mathfrak{l o g}\left(\dagger \mathcal{F}_{\underline{v}}\right)}
$$

[cf. [AbsTopIII], Definition 5.4, (iii)]. In particular, by applying the natural, algorithmically constructible topological field structure on $\Psi \frac{\mathrm{gp}}{\mathfrak{l o g}\left({ }^{\dagger} \mathcal{F}_{\underline{v}}\right)}[\mathrm{cf}$. [AbsTopIII], Proposition 3.2, (iii)], it thus follows that one may think of this log-shell as an object associated to the codomain of any [that is to say, not necessarily tautological!] log-link

$$
\dagger \mathcal{F}_{\underline{v}} \stackrel{\log }{\longrightarrow} \ddagger \mathcal{F}_{\underline{v}}
$$


- i.e., an object that is determined by the image of a certain portion [namely, the $G_{\underline{v}}\left({ }^{\dagger} \Pi_{\underline{v}}\right)$-invariants of $\left.\Psi_{\dagger \mathcal{F}_{\underline{v}}}^{\times}\right]$of the domain of this log-link.

(ii) Let $\underline{v} \in \underline{\mathbb{V}}^{\text {arc }}$. For $N \in \mathbb{N}_{\geq 1}$, write $\Psi_{\dagger \mathcal{F}_{\underline{v}}}^{\boldsymbol{\mu}_{N}} \subseteq \Psi_{\dagger \mathcal{F}_{\underline{v}}}^{\times} \subseteq \Psi_{\dagger \mathcal{F}_{\underline{v}}}^{\text {gp }}$ for the subgroup of $N$-th roots of unity and $\Psi_{\uparrow_{\mathcal{F}_{v}}}^{\sim} \rightarrow \Psi_{\Psi_{\mathcal{F}_{v}}}^{\mathrm{gp}}$ for the [pointed] universal covering of the topological group determined by the groupification $\Psi_{\uparrow_{\mathcal{F}_{v}}}^{\mathrm{gp}}$ of the topological monoid $\Psi_{\dagger \mathcal{F}_{\underline{v}}}$. Then one verifies immediately that one may think of the composite covering of topological groups

$$
\Psi_{\dagger \mathcal{F}_{\underline{v}}}^{\sim} \rightarrow \Psi_{\dagger \mathcal{F}_{\underline{v}}}^{\mathrm{gp}} \rightarrow \Psi_{\dagger \mathcal{F}_{\underline{v}}}^{\mathrm{gp}} / \Psi_{\dagger \mathcal{F}_{\underline{v}}}^{\mu_{N}}
$$

— where the second " $\rightarrow$ " is the natural surjection — as a [pointed] universal covering of $\Psi_{\dagger \mathcal{F}_{\underline{v}}}^{\mathrm{gp}} / \Psi_{\dagger \mathcal{F}_{\underline{v}}}^{\boldsymbol{\mu}_{N}}$. That is to say, one may think of $\Psi_{\dagger \mathcal{F}_{\underline{v}}}^{\sim}$ as an object constructed from $\Psi_{\dagger \mathcal{F}_{\underline{v}}}^{\mathrm{gp}} / \bar{\Psi}_{\uparrow_{\mathcal{F}_{\underline{v}}}}^{\boldsymbol{\mu}_{N}}[\overline{\mathrm{cf}}$. also Remark 1.2.1 below]. Now let us recall from the theory of [AbsTopIII] [cf. [AbsTopIII], Definition 4.1, (iv); [AbsTopIII], Proposition 4.2, (i), (ii)] that the natural, algorithmically constructible topological field structure on $\Psi_{\dagger \mathcal{F}_{\underline{v}}}^{\mathrm{gp}}$ allows one to define a [complex archimedean] logarithm on $\Psi_{\dagger \mathcal{F}_{\underline{v}}}^{\sim}$, which, in turn, yields a functorial algorithm in the collection of data ${ }^{\dagger} \mathcal{F}_{\underline{v}}$ [cf. [IUTchI], Definition 5.2, (i), (b)] for constructing a topological field structure on $\Psi_{\dagger \mathcal{F}_{\underline{v}}}^{\sim}$, together with a $\Psi_{\dagger_{\mathcal{F}_{\underline{v}}}^{\sim}}^{\sim}-$ Kummer structure on $\dagger_{\mathbb{U}_{\underline{v}}}[\mathrm{cf}$. [AbsTopIII], Definition 4.1, (iv); [IUTchII], Proposition 4.4, (i)]. Write

$$
\Psi_{\mathfrak{l o g}\left(\dagger \mathcal{F}_{\underline{v}}\right)} \subseteq \Psi_{\dagger \mathcal{F}_{\underline{v}}}^{\sim}
$$

for the resulting multiplicative monoid of nonzero integers. Here, we observe that the resulting diagram

$$
\Psi_{\dagger \mathcal{F}_{\underline{v}}} \subseteq \Psi_{\dagger \mathcal{F}_{\underline{v}}}^{\mathrm{gp}} \leftarrow \Psi_{\dagger \mathcal{F}_{\underline{v}}}^{\sim}=\Psi \frac{\mathrm{gp}}{\mathfrak{l o g}\left(\dagger \mathcal{F}_{\underline{v}}\right)}
$$

is compatible [cf. the discussion of [AbsTopIII], Definition 4.1, (iv)] with the co-holomorphicizations determined by the natural $\Psi_{\uparrow_{\mathcal{F}_{\underline{v}}}}^{\mathrm{gp}}$-Kummer [cf. [IUTchII], Proposition 4.4, (i)] and $\Psi_{\dagger \mathcal{F}_{\underline{v}}}^{\sim}-$ Kummer [cf. the above discussion] structures on $\dagger \mathbb{U}_{\underline{v}}$. The triple of data consisting of the topological monoid $\Psi_{\mathfrak{l o g}\left({ }^{\dagger} \mathcal{F}_{\underline{v}}\right)}$, the Autholomorphic space $\dagger^{\dagger} \mathbb{U}_{\underline{v}}$, and the $\Psi_{\dagger_{\mathcal{F}_{\underline{v}}}^{\sim}}^{\sim}$-Kummer structure on $\dagger^{\dagger} \mathbb{U}_{\underline{\underline{v}}}$ discussed above determines a collection of data [i.e., as in [IUTchI], Definition 5.2, (i), (b)]

$$
\log \left({ }^{\dagger} \mathcal{F}_{\underline{v}}\right)
$$

which is, in fact, naturally isomorphic to the collection of data ${ }^{\dagger} \mathcal{F}_{\underline{v}}$, but which we wish to think of as being related to ${ }^{\dagger} \mathcal{F}_{\underline{v}}$ via the above diagram. We shall denote this diagram by means of the notation

$$
\dagger \mathcal{F}_{\underline{v}} \stackrel{\log }{\longrightarrow} \log \left({ }^{\dagger} \mathcal{F}_{\underline{v}}\right)
$$

and refer to this relationship between ${ }^{\dagger} \mathcal{F}_{\underline{v}}$ and $\mathfrak{l o g}\left({ }^{\dagger} \mathcal{F}_{\underline{v}}\right)$ as the tautological loglink associated to ${ }^{\dagger} \mathcal{F}_{\underline{v}}$ [or, when $\dagger \mathfrak{F}$ is fixed, at $\left.\underline{v}\right]$. If $\log \left({ }^{\dagger} \mathcal{F}_{\underline{v}}\right) \stackrel{\sim}{\rightarrow} \ddagger \mathcal{F}_{\underline{v}}$ is any 
[poly-]isomorphism of collections of data [i.e., as in [IUTchI], Definition 5.2, (i), (b)], then we shall write

$$
\dagger \mathcal{F}_{\underline{v}} \stackrel{\log }{\longrightarrow} \ddagger \mathcal{F}_{\underline{v}}
$$

for the diagram obtained by post-composing the tautological log-link associated to ${ }^{\dagger} \mathcal{F}_{\underline{v}}$ with the given [poly-]isomorphism $\log \left({ }^{\dagger} \mathcal{F}_{\underline{v}}\right) \stackrel{\sim}{\rightarrow} \ddagger \mathcal{F}_{\underline{v}}$ and refer to this relationship between ${ }^{\dagger} \mathcal{F}_{\underline{v}}$ and $\ddagger \mathcal{F}_{\underline{v}}$ as a log-link from ${ }^{\dagger} \mathcal{F}_{\underline{v}}$ to $\ddagger \mathcal{F}_{\underline{v}}$; when the given [poly-]isomorphism $\log \left({ }^{\dagger} \mathcal{F}_{\underline{v}}\right) \stackrel{\sim}{\rightarrow} \ddagger \mathcal{F}_{\underline{v}}$ is the full poly-isomorphism, then we shall refer to the resulting $\mathfrak{l o g}$-link as the full $\mathfrak{l o g}$-link from ${ }^{\dagger} \mathcal{F}_{\underline{v}}$ to ${ }^{\ddagger} \mathcal{F}_{\underline{v}}$. Finally, we recall from [AbsTopIII], Definition 4.1, (iv), that the submonoid of units $\Psi_{\dagger \mathcal{F}_{\underline{v}}}^{\times} \subseteq \Psi_{\dagger \mathcal{F}_{\underline{v}}}$ determines a compact topological subquotient of $\Psi_{\Psi_{\mathcal{F}_{\underline{v}}}^{\sim}}^{\sim}$, which we shall refer to as the pre-log-shell. We shall refer to the $\Psi_{\mathfrak{l o g}\left({ }^{\dagger} \mathcal{F}_{v}\right)}^{\times}$-orbit of the [uniquely determined] closed line segment of $\Psi_{\uparrow_{\mathcal{F}_{\underline{v}}}^{\sim}}^{\sim}$ which is preserve $\bar{d}$ by multiplication by \pm 1 and whose endpoints differ by a generator of the kernel of the natural surjection $\Psi_{\dagger \mathcal{F}_{\underline{v}}}^{\sim} \rightarrow \Psi_{\uparrow \mathcal{F}_{\underline{v}}}^{\mathrm{gp}}$ — or, equivalently, the $\Psi_{\mathfrak{l o g}\left(\dagger \mathcal{F}_{\underline{v}}\right)}^{\times}$-orbit of the result of multiplying by $N$ the [uniquely determined] closed line segment of $\Psi_{\Psi_{\mathcal{F}_{\underline{v}}}^{\sim}}^{\sim}$ which is preserved by multiplication by \pm 1 and whose endpoints differ by a generator of the kernel of the natural surjection $\Psi_{\dagger \mathcal{F}_{\underline{v}}}^{\sim} \rightarrow \Psi_{\dagger \mathcal{F}_{\underline{v}}}^{\mathrm{gp}} / \Psi_{\dagger \mathcal{F}_{\underline{v}}}^{\boldsymbol{\mu}_{N}}$ - as the log-shell

$$
\mathcal{I}_{\dagger \mathcal{F}_{\underline{v}}} \subseteq \Psi_{\dagger \mathcal{F}_{\underline{v}}}^{\sim}=\Psi \frac{\mathrm{gp}}{\mathfrak{l o g}\left(\dagger \mathcal{F}_{\underline{v}}\right)}
$$

[cf. [AbsTopIII], Definition 5.4, (v)]. Thus, one may think of the log-shell as an object constructed from $\Psi_{\uparrow \mathcal{F}_{v}}^{\mathrm{gp}} / \Psi_{\dagger \mathcal{F}_{v}}^{\boldsymbol{\mu}_{N}}$. Moreover, by applying the natural, algorithmically constructible topological field structure on $\Psi \frac{\mathrm{gp}}{\mathfrak{l o g}\left(\dagger \mathcal{F}_{\underline{v}}\right)}\left(=\Psi_{\dagger \mathcal{F}_{\underline{v}}}^{\sim}\right)$, it thus follows that one may think of this log-shell as an object associated to the codomain of any [that is to say, not necessarily tautological!] log-link

$$
\dagger \mathcal{F}_{\underline{v}} \stackrel{\log }{\longrightarrow} \ddagger \mathcal{F}_{\underline{v}}
$$

- i.e., an object that is determined by the image of a certain portion [namely, the subquotient $\Psi_{\dagger \mathcal{F}_{\underline{v}}}^{\times}$of $\left.\Psi_{\dagger \mathcal{F}_{\underline{\underline{v}}}}^{\sim}\right]$ of the domain of this log-link.

(iii) Write

$$
\underline{\mathfrak{l o g}}\left({ }^{\dagger} \mathfrak{F}\right) \stackrel{\text { def }}{=}\left\{\underline{\mathfrak{l o g}}\left({ }^{\dagger} \mathcal{F}_{\underline{v}}\right) \stackrel{\text { def }}{=} \Psi_{\dagger \mathcal{F}_{\underline{v}}}^{\sim}\right\}_{\underline{v} \in \underline{\mathbb{V}}}
$$

for the collection of topological modules constructed in (i), (ii) above indexed by $\underline{v} \in \mathbb{V}$ - where the group structure arises from the additive portion of the field structures on $\Psi_{\dagger \mathcal{F}_{\underline{v}}}^{\sim}$ discussed in (i), (ii); for $\underline{v} \in \underline{\mathbb{V}}^{\text {non }}$, we regard $\Psi_{\uparrow_{\mathcal{F}_{\underline{v}}}^{\sim}}$ as equipped with its natural $G_{\underline{v}}\left({ }^{\dagger} \Pi_{\underline{v}}\right)$-action. Write

$$
\mathfrak{l o g}\left({ }^{\dagger} \mathfrak{F}\right) \stackrel{\text { def }}{=}\left\{\mathfrak{l o g}\left({ }^{\dagger} \mathcal{F}_{\underline{v}}\right)\right\}_{\underline{v} \in \underline{\mathbb{V}}}
$$

for the $\mathcal{F}$-prime-strip determined by the data $\log \left({ }^{\dagger} \mathcal{F}_{\underline{v}}\right)$ constructed in (i), (ii) for $\underline{v} \in \underline{\mathbb{V}}$. We shall denote by

$$
{ }^{\dagger} \mathfrak{F} \quad \stackrel{\mathfrak{l o g}}{\longrightarrow} \log \left({ }^{\dagger} \mathfrak{F}\right)
$$


the collection of diagrams $\left\{{ }^{\dagger} \mathcal{F}_{\underline{v}} \stackrel{\mathfrak{l o g}}{\longrightarrow} \mathfrak{l o g}\left({ }^{\dagger} \mathcal{F}_{\underline{v}}\right)\right\}_{\underline{v} \in \underline{\mathbb{V}}}$ constructed in (i), (ii) for $\underline{v} \in \underline{\mathbb{V}}$ and refer to this relationship between ${ }^{\dagger} \mathfrak{F}$ and $\mathfrak{l o g}\left({ }^{\dagger} \mathfrak{F}\right)$ as the tautological log-link associated to ${ }^{\dagger} \mathfrak{F}$. If $\log \left({ }^{\dagger} \mathfrak{F}\right) \stackrel{\sim}{\rightarrow} \ddagger \mathfrak{F}$ is any [poly-]isomorphism of $\mathcal{F}$-prime-strips, then we shall write

$$
\dagger \mathfrak{F} \stackrel{\log }{\longrightarrow} \ddagger \mathfrak{F}
$$

for the diagram obtained by post-composing the tautological $\mathfrak{l o g}$-link associated to ${ }^{\dagger} \mathfrak{F}$ with the given [poly-]isomorphism $\log \left({ }^{\dagger} \mathfrak{F}\right) \stackrel{\sim}{\rightarrow}{ }^{\ddagger} \mathfrak{F}$ and refer to this relationship between ${ }^{\dagger} \mathfrak{F}$ and ${ }^{\ddagger} \mathfrak{F}$ as a log-link from ${ }^{\dagger} \mathfrak{F}$ to ${ }^{\ddagger} \mathfrak{F}$; when the given [poly-]isomorphism $\mathfrak{l o g}\left({ }^{\dagger} \mathfrak{F}\right) \stackrel{\sim}{\rightarrow}{ }^{\ddagger} \mathfrak{F}$ is the full poly-isomorphism, then we shall refer to the resulting loglink as the full $\mathfrak{l o g}$-link from ${ }^{\dagger} \mathfrak{F}$ to ${ }^{\ddagger} \mathfrak{F}$. Finally, we shall write

$$
\mathcal{I}_{\dagger \mathfrak{F}} \stackrel{\text { def }}{=}\left\{\mathcal{I}_{\dagger \mathcal{F}_{\underline{v}}}\right\}_{\underline{v} \in \underline{\mathbb{V}}}
$$

for the collection of log-shells constructed in (i), (ii) for $\underline{v} \in \underline{\mathbb{V}}$ and refer to this collection as the log-shell associated to ${ }^{\dagger} \mathfrak{F}$ and [by a slight abuse of notation]

$$
\mathcal{I}_{\dagger} \mathfrak{F} \subseteq \underline{\log }\left({ }^{\dagger} \mathfrak{F}\right)
$$

for the collection of natural inclusions indexed by $\underline{v} \in \underline{\mathbb{V}}$. In particular, [cf. the discussion of (i), (ii)], it thus follows that one may think of this log-shell as an object associated to the codomain of any [that is to say, not necessarily tautological!] loglink

$$
\dagger \mathfrak{F} \stackrel{\mathfrak{l o g}}{\longrightarrow} \ddagger \mathfrak{F}
$$

- i.e., an object that is determined by the image of a certain portion [cf. the discussion of (i), (ii)] of the domain of this log-link.

(iv) Let $\underline{v} \in \underline{\mathbb{V}}^{\text {non }}$. Then observe that it follows immediately from the constructions of (i) that the topological modules with $G_{\underline{v}}\left({ }^{\dagger} \Pi_{\underline{v}}\right)$-action $\mathcal{I}_{\dagger} \mathcal{F}_{\underline{v}} \subseteq \underline{\mathfrak{l o g}}\left({ }^{\dagger} \mathcal{F}_{\underline{v}}\right)$ may be constructed solely from the collection of data ${ }^{\dagger} \mathcal{F}_{\underline{v}}^{+\times \mu}$ [i.e., the portion of the $\mathcal{F}^{\vdash \times \boldsymbol{\mu}}$-prime-strip $\mathfrak{F}^{\vdash \times \boldsymbol{\mu}}$ labeled by $\underline{v}$ ]. That is to say, in light of the definition of a $\times \boldsymbol{\mu}$-Kummer structure [cf. [IUTchII], Definition 4.9, (i), (ii), (iv), (vi), (vii)], these constructions only require the perfection "(-) pf " of the units and are manifestly unaffected by the operation of forming the quotient by a torsion subgroup of the units. Write

$$
\mathcal{I}_{\dagger \mathcal{F}_{\underline{v}}^{\vdash \times \mu}} \subseteq \underline{\log }\left({ }^{\dagger} \mathcal{F}_{\underline{v}}^{\vdash \times \mu}\right)
$$

for the resulting topological modules with $G_{\underline{v}}\left({ }^{\dagger} \Pi_{\underline{v}}\right)$-action, regarded as objects constructed from ${ }^{\dagger} \mathcal{F}_{\underline{v}}^{\vdash} \times \boldsymbol{\mu}$.

(v) Let $\underline{v} \in \underline{\mathbb{V}}^{\text {arc }}$. Then by applying the algorithms for constructing " $k \sim(G)$ ", "I $(G)$ " given in [AbsTopIII], Proposition 5.8, (v), to the [object of the category " $\mathbb{T M}^{\vdash}$ " of split topological monoids discussed in [IUTchI], Example 3.4, (ii), determined by the] split Frobenioid portion of the collection of data ${ }^{\dagger} \mathcal{F}_{\underline{v}}$, one obtains a functorial algorithm in the collection of data ${ }^{\dagger} \mathcal{F}_{\underline{v}}^{+}$for constructing a topological module $\underline{\mathfrak{l o g}}\left({ }^{\dagger} \mathcal{F}_{\underline{v}}^{+}\right)$[i.e., corresponding to " $k^{\sim}(G)$ "] and a topological subspace $\mathcal{I}_{\dagger \mathcal{F}_{v}}$ [i.e., corresponding to " $\mathcal{I}(G)$ "]. In fact, this functorial algorithm only makes use of the unit portion of this split Frobenioid, together with a pointed universal covering 
of this unit portion. Moreover, by arguing as in (ii), one may in fact regard this functorial algorithm as an algorithm that only makes use of the quotient of this unit portion by its $N$-torsion subgroup, for $N \in \mathbb{N}_{\geq 1}$, together with a pointed universal covering of this quotient. That is to say, this functorial algorithm may, in fact, be regarded as a functorial algorithm in the collection of data ${ }^{\dagger} \mathcal{F}_{\underline{v}}^{+} \times \boldsymbol{\mu}$. Write

$$
\mathcal{I}_{\dagger \mathcal{F}_{\underline{v}}^{\vdash \times \mu}} \subseteq \underline{\log }\left({ }^{\dagger} \mathcal{F}_{\underline{v}}^{\vdash \times \mu}\right)
$$

for the resulting topological module equipped with a closed subspace, regarded as objects constructed from ${ }^{\dagger} \mathcal{F}_{\underline{v}}^{\vdash \times \mu}$.

(vi) Finally, just as in (iii), we shall write

$$
\mathcal{I}_{\dagger \mathfrak{F}^{\vdash \times \mu}} \stackrel{\text { def }}{=}\left\{\mathcal{I}_{\dagger \mathcal{F}_{\underline{v}}^{\vdash \times \mu}}\right\}_{\underline{v} \in \underline{\mathbb{V}}} \subseteq \underline{\mathfrak{l o g}}\left({ }^{\dagger} \mathfrak{F}^{\vdash \times \boldsymbol{\mu}}\right) \stackrel{\text { def }}{=} \quad\left\{\underline{\mathfrak{l o g}}\left({ }^{\dagger} \mathcal{F}_{\underline{v}}^{\vdash \times \mu}\right)\right\}_{\underline{v} \in \underline{\mathbb{V}}}
$$

for the resulting collections of data constructed solely from the $\mathcal{F}^{\vdash \times \boldsymbol{\mu}}$-prime-strip ${ }^{\dagger} \mathfrak{F}^{\vdash \times \boldsymbol{\mu}}$ [i.e., which we do not regard as objects constructed from $\left.{ }^{\dagger} \mathfrak{F} !\right]$.

From the point of view of the present series of papers, the theory of [AbsTopIII] may be summarized as follows.

\section{Proposition 1.2. (log-links Between $\mathcal{F}$-prime-strips) Let}

$$
{ }^{\dagger} \mathfrak{F}=\left\{{ }^{\dagger} \mathcal{F}_{\underline{v}}\right\}_{\underline{v} \in \underline{\mathbb{V}}} ; \quad{ }^{\ddagger} \mathfrak{F}=\left\{{ }^{\ddagger} \mathcal{F}_{\underline{v}}\right\}_{\underline{v} \in \underline{\mathbb{V}}}
$$

be $\mathcal{F}$-prime-strips [relative to the given initial $\Theta$-data $-c f$. [IUTchI], Definition 5.2, (i)] and

$$
{ }^{\dagger} \mathfrak{F} \stackrel{\mathfrak{l o g}}{\longrightarrow} \ddagger \mathfrak{F}
$$

$a$ log-link from ${ }^{\dagger} \mathfrak{F}$ to ${ }^{\ddagger} \mathfrak{F}$. Write ${ }^{\dagger} \mathfrak{F}^{\vdash \times \boldsymbol{\mu}},{ }^{\ddagger} \mathfrak{F}^{\vdash \times \boldsymbol{\mu}}$ for the associated $\mathcal{F}^{\vdash \times \boldsymbol{\mu}}$-prime-strips [cf. [IUTchII], Definition 4.9, (vi), (vii)]; ${ }^{\dagger} \mathfrak{D},{ }^{\ddagger} \mathfrak{D}$ for the associated $\mathcal{D}$-prime-strips [cf. [IUTchI], Remark 5.2.1, (i)]; ${ }^{\dagger} \mathfrak{D}^{\vdash},{ }^{\ddagger} \mathfrak{D}^{\vdash}$ for the associated $\mathcal{D}^{\vdash}$-prime-strips [cf. [IUTchI], Definition 4.1, (iv)]. Also, let us recall the diagrams

$$
\begin{aligned}
& \Psi_{\dagger \mathcal{F}_{\underline{v}}} \supseteq \Psi_{\dagger \mathcal{F}_{\underline{v}}}^{\times} \rightarrow \underline{\log }\left({ }^{\dagger} \mathcal{F}_{\underline{v}}\right)=\Psi \frac{\mathrm{gp}}{\mathfrak{l o g}\left(\dagger \mathcal{F}_{\underline{v}}\right)} \stackrel{\sim}{\rightarrow} \Psi_{\ddagger \mathcal{F}_{\underline{v}}}^{\underline{g p}} \quad\left(*_{\text {non }}\right) \\
& \Psi_{\dagger \mathcal{F}_{\underline{v}}} \subseteq \Psi_{\dagger \mathcal{F}_{\underline{v}}}^{\mathrm{gp}} \nleftarrow \underline{\mathfrak{l o g}}\left({ }^{\dagger} \mathcal{F}_{\underline{v}}\right)=\Psi \frac{\mathrm{gp}}{\mathfrak{l o g}\left(\dagger \mathcal{F}_{\underline{v}}\right)} \stackrel{\sim}{\rightarrow} \Psi_{\ddagger \mathcal{F}_{\underline{v}}}^{\underline{\mathrm{gp}}} \quad\left(*_{\operatorname{arc}}\right)
\end{aligned}
$$

- where the $\underline{v}$ of $\left(*_{\text {non }}\right)$ (respectively, $\left.\left(*_{\operatorname{arc}}\right)\right)$ belongs to $\underline{\mathbb{V}}^{\text {non }}$ (respectively, $\left.\underline{\mathbb{V}}^{\text {arc }}\right)$, and the [poly-]isomorphisms on the right are induced by the " $\stackrel{\log }{\longrightarrow}$ " - of Definition 1.1, (i), (ii).

(i) (Coricity of Associated D-Prime-Strips) The log-link ${ }^{\dagger} \mathfrak{F} \stackrel{\text { log }}{\longrightarrow} \quad \mathfrak{F}$ induces [poly-]isomorphisms

$$
\dagger \mathfrak{D} \stackrel{\sim}{\rightarrow} \ddagger \mathfrak{D} ; \quad{ }^{\dagger} \mathfrak{D}^{\vdash} \stackrel{\sim}{\rightarrow}{ }^{\ddagger} \mathfrak{D}^{\vdash}
$$


between the associated $\mathcal{D}$ - and $\mathcal{D}^{\vdash}$-prime-strips. In particular, the [poly-]isomorphism $\dagger \mathfrak{D} \stackrel{\sim}{\rightarrow} \ddagger \mathfrak{D}$ induced by ${ }^{\dagger} \mathfrak{F} \stackrel{\text { log }}{\longrightarrow} \ddagger \mathfrak{F}$ induces a [poly-]isomorphism

$$
\Psi_{\mathrm{cns}}\left({ }^{\dagger} \mathfrak{D}\right) \stackrel{\sim}{\rightarrow} \Psi_{\mathrm{cns}}\left({ }^{\ddagger} \mathfrak{D}\right)
$$

between the collections of monoids equipped with auxiliary data of [IUTchII], Corollary 4.5, (i).

(ii) (Simultaneous Compatibility with Ring Structures) At $\underline{v} \in \underline{\mathbb{V}}^{\text {non }}$, the natural ${ }^{\dagger} \Pi_{\underline{\underline{v}}}$-actions on the " $\Psi$ 's" appearing in the diagram $\left(*_{\mathrm{non}}\right)$ are compatible with the topological ring structures on $\Psi \frac{\mathrm{gp}}{\dagger_{\mathcal{F}_{\underline{v}}}}$ and $\Psi \frac{\mathrm{gp}}{\mathfrak{l o g}\left(\dagger \mathcal{F}_{\underline{v}}\right)}$. At $\underline{v} \in \underline{\mathbb{V}}^{\text {arc }}$, the co-holomorphicizations determined by the natural $\Psi \frac{\mathrm{gp}}{\dagger_{\mathcal{F}_{\underline{v}}}}-$ and $\Psi \frac{\mathrm{gp}}{\mathfrak{l o g}\left(\dagger \mathcal{F}_{\underline{v}}\right)}(=$ $\Psi_{\dagger \mathcal{F}_{v}}^{\sim}$ )-Kummer structures on $\dagger_{\mathbb{U}_{\underline{v}}}^{\sim}$ - which [cf. the discussion of Definition 1.1, (ii)] are compatible with the diagram $\left(*_{\text {arc }}\right)$ - are compatible with the topological ring structures on $\Psi \frac{\mathrm{gp}}{\dagger \mathcal{F}_{\underline{v}}}$ and $\Psi \frac{\mathrm{gp}}{\mathfrak{l o g}\left(\dagger \mathcal{F}_{\underline{\underline{v}}}\right)}$.

(iii) (Simultaneous Compatibility with Log-volumes) $A t \underline{v} \in \underline{\mathbb{V}}^{\text {non }}$, the diagram $\left(*_{\text {non }}\right)$ is compatible with the natural $p_{v}$-adic log-volumes $[c f . \quad[A b$ sTopIII], Proposition 5.7, (i); [AbsTopIII], Corollary 5.10, (ii)] on the subsets of ${ }^{\dagger} \Pi_{\underline{\underline{v}}}$-invariants of $\Psi \frac{\mathrm{gp}}{\dagger_{\mathcal{F}}}$ and $\Psi \frac{\mathrm{gp}}{\mathfrak{l o g}\left(\dagger \mathcal{F}_{\underline{v}}\right)}$. At $\underline{v} \in \underline{\mathbb{V}}^{\text {arc }}$, the diagram (*arc) is compatible with the natural angular log-volume [cf. Remark 1.2.1 below; [AbsTopIII], Proposition 5.7, (ii); [AbsTopIII], Corollary 5.10, (ii)] on $\Psi_{+\mathcal{F}_{v}}^{\times}$and the natural radial log-volume [cf. [AbsTopIII], Proposition 5.7, (ii); [Abs TopIII], Corollary 5.10, (ii)] on $\Psi \frac{\mathrm{gp}}{\mathfrak{l o g}\left(\dagger \mathcal{F}_{\underline{v}}\right)}$.

(iv) (Kummer theory) The Kummer isomorphisms

$$
\Psi_{\mathrm{cns}}\left({ }^{\dagger} \mathfrak{F}\right) \stackrel{\sim}{\rightarrow} \quad \Psi_{\mathrm{cns}}\left({ }^{\dagger} \mathfrak{D}\right) ; \quad \Psi_{\mathrm{cns}}\left({ }^{\ddagger} \mathfrak{F}\right) \stackrel{\sim}{\rightarrow} \quad \Psi_{\mathrm{cns}}\left({ }^{\ddagger} \mathfrak{D}\right)
$$

of [IUTchII], Corollary 4.6, (i), fail to be compatible with the [poly-]isomorphism $\Psi_{\mathrm{cns}}(\dagger \mathfrak{D}) \stackrel{\sim}{\rightarrow} \Psi_{\mathrm{cns}}\left({ }^{\ddagger} \mathfrak{D}\right)$ of $(i)$, relative to the diagrams $\left(*_{\mathrm{non}}\right),\left(*_{\text {arc }}\right)$ land the notational conventions of Definition 1.1] - cf. [AbsTopIII], Corollary 5.5, (iv). [Here, we regard the diagrams $\left(*_{\text {non }}\right),\left(*_{\text {arc }}\right)$ as diagrams that relate $\Psi_{\dagger \mathcal{F}_{\underline{v}}}$ and $\Psi_{\ddagger \mathcal{F}_{\underline{v}}}$, via the [poly-]isomorphism $\mathfrak{l o g}\left({ }^{\dagger} \mathfrak{F}\right) \stackrel{\sim}{\rightarrow} \ddagger \mathfrak{F}$ that determines the $\log$-link ${ }^{\dagger} \mathfrak{F} \stackrel{\mathfrak{l o g}}{\longrightarrow}{ }^{\ddagger} \mathfrak{F}$.]

(v) (Holomorphic Log-shells) At $\underline{v} \in \underline{\mathbb{V}}^{\text {non }}$, the log-shell

$$
\mathcal{I}_{\dagger \mathcal{F}_{\underline{v}}} \subseteq \underline{\log }\left({ }^{\dagger} \mathcal{F}_{\underline{v}}\right) \quad\left(\stackrel{\sim}{\rightarrow} \quad \Psi \frac{\mathrm{gp}}{\ddagger \mathcal{F}_{\underline{v}}}\right)
$$

satisfies the following properties: $\left(a_{\text {non }}\right) \mathcal{I}_{\dagger \mathcal{F}_{v}}$ is compact, hence of finite logvolume [cf. [AbsTopIII], Corollary 5.10, $(i) \overline{]} ;\left(b_{\text {non }}\right) \mathcal{I}_{\dagger \mathcal{F}_{\underline{v}}}$ contains the submonoid of ${ }^{\dagger} \Pi_{\underline{\underline{v}}}$-invariants of $\Psi_{\mathfrak{l o g}\left(\dagger \mathcal{F}_{\underline{v}}\right)}$ [cf. [AbsTopIII], Definition 5.4, (iii)]; (c $\left.c_{\mathrm{non}}\right) \mathcal{I}_{\dagger \mathcal{F}_{\underline{v}}}$ contains the image of the submonoid of ${ }^{\dagger} \Pi_{\underline{\underline{v}}}$-invariants of $\Psi_{\uparrow_{\mathcal{F}_{\underline{v}}}}^{\times}$. At $\underline{v} \in \underline{\mathbb{V}}^{\text {arc }}$, the log-shell

$$
\mathcal{I}_{\dagger \mathcal{F}_{\underline{\underline{v}}}} \subseteq \underline{\log }\left({ }^{\dagger} \mathcal{F}_{\underline{\underline{v}}}\right) \quad\left(\stackrel{\sim}{\rightarrow} \quad \Psi_{\ddagger \underline{\mathcal{F}_{\underline{v}}}}^{\mathrm{gp}}\right)
$$


satisfies the following properties: $\left(a_{\mathrm{arc}}\right) \mathcal{I}_{\dagger \mathcal{F}_{v}}$ is compact, hence of finite radial log-volume [cf. [AbsTopIII], Corollary 5.10, (i)]; (barc $) \mathcal{I}_{\dagger \mathcal{F}_{\underline{v}}}$ contains $\Psi_{\mathfrak{l o g}\left(\dagger \mathcal{F}_{\underline{v}}\right)}$ [cf. [AbsTopIII], Definition 5.4, (v)]; (carc $)$ the image of $\mathcal{I}_{\mathcal{F}_{\underline{\underline{v}}}}$ in $\Psi_{+\mathcal{F}_{\underline{v}}}^{\mathrm{gp}}$ contains $\Psi_{\dagger \mathcal{F}_{\underline{\underline{v}}}}^{\times}[$i.e., in essence, the pre-log-shell].

(vi) (Nonarchimedean Mono-analytic Log-shells) At $\underline{v} \in \underline{\mathbb{V}}^{\text {non }}$, if we write ${ }^{\dagger} \mathcal{D}_{v}^{\vdash}=\mathcal{B}^{\operatorname{temp}}\left({ }^{\dagger} G_{\underline{v}}\right)^{0}$ for the portion of ${ }^{\dagger} \mathfrak{D}^{\vdash}$ indexed by $\underline{v}$ [cf. the notation of [IUTChII], Corollary 4.5], then the algorithms for constructing " $\sim^{\sim}(G)$ ", "I $(G)$ " given in [AbsTopIII], Proposition 5.8, (ii), yield a functorial algorithm in the category ${ }^{\dagger} \mathcal{D}_{\underline{v}}^{+}$for constructing a topological module equipped with a continuous ${ }^{\dagger} G_{\underline{v}^{-}}$ action

$$
\underline{\mathfrak{l o g}}\left({ }^{\dagger} \mathcal{D}_{\underline{v}}^{\vdash}\right) \stackrel{\text { def }}{=}\left\{{ }^{\dagger} G_{\underline{v}} \curvearrowright k^{\sim}\left({ }^{\dagger} G_{\underline{v}}\right)\right\}
$$

and a topological submodule — i.e., a "mono-analytic log-shell" -

$$
\mathcal{I}_{\dagger \mathcal{D}_{\underline{v}}^{\vdash}} \stackrel{\text { def }}{=} \mathcal{I}\left({ }^{\dagger} G_{\underline{v}}\right) \subseteq k^{\sim}\left({ }^{\dagger} G_{\underline{v}}\right)
$$

equipped with a $p_{\underline{v}}$-adic log-volume [cf. [AbsTopIII], Corollary 5.10, (iv)]. Moreover, there is a natural functorial algorithm [cf. the second display of [IUTchII], Corollary 4.6, (ii)] in the collection of data ${ }^{\dagger} \mathcal{F}_{\underline{v}}^{\vdash \times \boldsymbol{\mu}}$ [i.e., the portion of ${ }^{\dagger} \mathfrak{F}^{\vdash \times \boldsymbol{\mu}}$ labeled by $\underline{v}$ ] for constructing an Ism-orbit of isomorphisms [cf. [IUTchII], Example 1.8, (iv); [IUTchII], Definition 4.9, (i), (vii)]

$$
\underline{\mathfrak{l o g}}\left({ }^{\dagger} \mathcal{D}_{\underline{v}}^{\vdash}\right) \stackrel{\sim}{\rightarrow} \underline{\log }\left({ }^{\dagger} \mathcal{F}_{\underline{v}}^{+\times \mu}\right)
$$

of topological modules [cf. Definition 1.1, (iv)], as well as a functorial algorithm [cf. [AbsTopIII], Corollary 5.10, (iv), (c), (d); the fourth display of [IUTchII], Corollary 4.5, (ii); the final display of [IUTchII], Corollary 4.6, (i)] in the collection of data ${ }^{\dagger} \mathcal{F}_{\underline{v}}$ for constructing isomorphisms

$$
\underline{\log }\left({ }^{\dagger} \mathcal{D}_{\underline{v}}^{\vdash}\right) \stackrel{\sim}{\rightarrow} \underline{\log }\left({ }^{\dagger} \mathcal{F}_{\underline{v}}^{\vdash \times \mu}\right) \stackrel{\sim}{\rightarrow} \underline{\log }\left({ }^{\dagger} \mathcal{F}_{\underline{v}}\right) \quad\left(\stackrel{\sim}{\rightarrow} \quad \Psi \frac{\mathrm{gp}}{\ddagger \mathcal{F}_{\underline{v}}}\right)
$$

of topological modules. The various isomorphisms of the last two displays are compatible with one another, as well as with the respective ${ }^{\dagger} G_{\underline{v}^{-}}$and $G_{\underline{v}}\left({ }^{\dagger} \Pi_{\underline{v}}\right)$-actions Irelative to the natural identification ${ }^{\dagger} G_{\underline{v}}=G_{\underline{v}}\left({ }^{\dagger} \Pi_{\underline{v}}\right)$ that arises from regarding ${ }^{\dagger} \mathcal{D}_{\underline{v}}^{\vdash}$ as an object constructed from $\left.{ }^{\dagger} \mathcal{F}_{\underline{v}}^{\vdash \times \boldsymbol{\mu}}\right]$, the respective log-shells, and the respective log-volumes on these log-shells.

(vii) (Archimedean Mono-analytic Log-shells) At $\underline{v} \in \underline{\mathbb{V}}^{\text {arc }}$, the algorithms for constructing " $k^{\sim}(G)$ ", " $\mathcal{I}(G)$ " given in [AbsTopIII], Proposition 5.8, $(v)$, yield a functorial algorithm in ${ }^{\dagger} \mathcal{D}_{\underline{v}}^{+}$rregarded as an object of the category "TMM " of split topological monoids discussed in [IUTchI], Example 3.4, (ii)] for constructing a topological module

$$
\underline{\log }\left({ }^{\dagger} \mathcal{D}_{\underline{v}}^{\vdash}\right) \stackrel{\text { def }}{=} k^{\sim}\left({ }^{\dagger} G_{\underline{v}}\right)
$$

and a topological subspace — i.e., a "mono-analytic log-shell" -

$$
\mathcal{I}_{\dagger \mathcal{D}_{\underline{v}}^{\vdash}} \stackrel{\text { def }}{=} \mathcal{I}\left({ }^{\dagger} G_{\underline{v}}\right) \subseteq k^{\sim}\left({ }^{\dagger} G_{\underline{v}}\right)
$$


equipped with angular and radial log-volumes [cf. [AbsTopIII], Corollary 5.10, (iv)]. Moreover, there is a natural functorial algorithm [cf. the second display of [IUTchII], Corollary 4.6, (ii)] in the collection of data ${ }^{\dagger} \mathcal{F}_{\underline{v}}^{-} \times \boldsymbol{\mu}$ for constructing a poly-isomorphism [i.e., an orbit of isomorphisms with respect to the independent actions of $\{ \pm 1\}$ on each of the direct factors that occur in the construction of [AbsTopIII], Proposition 5.8, (v)]

$$
\underline{\mathfrak{l o g}}\left({ }^{\dagger} \mathcal{D}_{\underline{v}}^{\vdash}\right) \stackrel{\sim}{\rightarrow} \underline{\mathfrak{l o g}}\left({ }^{\dagger} \mathcal{F}_{\underline{v}}^{+} \times \boldsymbol{\mu}\right)
$$

of topological modules [cf. Definition 1.1, (v)], as well as a functorial algorithm [cf. [AbsTopIII], Corollary 5.10, (iv), (c), (d); the fourth display of [IUTchII], Corollary 4.5, (ii); the final display of [IUTchII], Corollary 4.6, (i)] in the collection of data ${ }^{\dagger} \mathcal{F}_{\underline{v}}$ for constructing poly-isomorphisms [i.e., orbits of isomorphisms with respect to the independent actions of $\{ \pm 1\}$ on each of the direct factors that occur in the construction of [AbsTopIII], Proposition 5.8, (v)]

$$
\underline{\mathfrak{l o g}}\left({ }^{\dagger} \mathcal{D}_{\underline{v}}^{\vdash}\right) \stackrel{\sim}{\rightarrow} \underline{\mathfrak{l o g}}\left({ }^{\dagger} \mathcal{F}_{\underline{v}}^{\vdash \times \mu}\right) \stackrel{\sim}{\rightarrow} \underline{\mathfrak{l o g}}\left({ }^{\dagger} \mathcal{F}_{\underline{v}}\right) \quad\left(\stackrel{\sim}{\rightarrow} \quad \Psi_{\ddagger}^{\underline{\mathrm{gp}}} \mathcal{F}_{\underline{v}}\right)
$$

of topological modules. The various isomorphisms of the last two displays are compatible with one another, as well as with the respective log-shells and the respective angular and radial log-volumes on these log-shells.

(viii) (Mono-analytic Log-shells) The various [poly-]isomorphisms of (vi), (vii) [cf. also Definition 1.1, (iii), (vi)] yield collections of [poly-]isomorphisms indexed by $\underline{v} \in \underline{\mathbb{V}}$

$$
\begin{aligned}
& \underline{\log }\left({ }^{\dagger} \mathfrak{D}^{\vdash}\right) \stackrel{\text { def }}{=}\left\{\underline{\log }\left({ }^{\dagger} \mathcal{D}_{\underline{v}}^{\vdash}\right)\right\}_{\underline{v} \in \underline{\mathbb{V}}} \stackrel{\sim}{\rightarrow} \underline{\log }\left({ }^{\dagger} \mathfrak{F}^{\vdash \times \boldsymbol{\mu}}\right) \stackrel{\text { def }}{=}\left\{\underline{\log }\left({ }^{\dagger} \mathcal{F}_{\underline{v}}^{\vdash \times \boldsymbol{\mu}}\right)\right\}_{\underline{v} \in \underline{\mathbb{V}}} \\
& \mathcal{I}_{\dagger \mathfrak{D}^{\vdash}} \stackrel{\text { def }}{=}\left\{\mathcal{I}_{\dagger \mathcal{D}_{\underline{v}}^{\vdash}}\right\}_{\underline{v} \in \underline{\mathbb{V}}} \stackrel{\sim}{\rightarrow} \mathcal{I}_{\dagger \mathfrak{F}^{\vdash \times \mu}} \stackrel{\text { def }}{=}\left\{\mathcal{I}_{\dagger \mathcal{F}_{\underline{v}}^{\vdash \times \mu}}\right\}_{\underline{v} \in \underline{\mathbb{V}}} \\
& \underline{\mathfrak{l o g}}\left({ }^{\dagger} \mathfrak{D}^{\vdash}\right) \stackrel{\sim}{\rightarrow} \underline{\mathfrak{l o g}}\left({ }^{\dagger} \mathfrak{F} \times \boldsymbol{\mu}\right) \stackrel{\sim}{\rightarrow} \underline{\mathfrak{l o g}}\left({ }^{\dagger} \mathfrak{F}\right) \stackrel{\text { def }}{=}\left\{\underline{\mathfrak{l o g}}\left({ }^{\dagger} \mathcal{F}_{\underline{v}}\right)\right\}_{\underline{v} \in \underline{\mathbb{V}}} \\
& \left(\stackrel{\sim}{\rightarrow} \Psi \frac{\mathrm{gp}}{\mathrm{cns}}\left({ }^{\ddagger} \mathfrak{F}\right) \stackrel{\text { def }}{=}\left\{\Psi_{\ddagger \mathcal{F}_{\underline{v}}}^{\underline{\mathrm{gp}}}\right\}_{\underline{v}} \in \underline{\mathbb{V}}\right) \\
& \mathcal{I}_{\dagger \mathfrak{D}^{\vdash}} \stackrel{\sim}{\rightarrow} \mathcal{I}_{\dagger \mathfrak{F}^{\vdash \times \mu}} \stackrel{\sim}{\rightarrow} \mathcal{I}_{\dagger \mathfrak{F}} \stackrel{\text { def }}{=}\left\{\mathcal{I}_{\dagger \mathcal{F}_{\underline{v}}}\right\}_{\underline{v} \in \underline{\mathbb{V}}}
\end{aligned}
$$

- where, in the definition of " $\Psi \frac{\mathrm{gp}}{\mathrm{cns}}\left({ }^{\ddagger} \mathfrak{F}\right)$ ", we regard each $\Psi_{\ddagger \mathcal{F}_{v}}^{\mathrm{gp}}$, for $\underline{v} \in \underline{\mathbb{V}}^{\text {non }}$, as being equipped with its natural $G_{\underline{v}}\left({ }^{\ddagger} \Pi_{\underline{v}}\right)$-action [cf. the discussion at the beginning of Definition 1.1].

(ix) (Coric Holomorphic Log-shells) Let $* \mathfrak{D}$ be a $\mathcal{D}$-prime-strip; write

$$
\mathfrak{F}\left({ }^{*} \mathfrak{D}\right)
$$

for the $\mathcal{F}$-prime-strip naturally determined by $\Psi_{\text {cns }}\left({ }^{*} \mathfrak{D}\right)$ [cf. [IUTchII], Remark 4.5.1, (i)]. Suppose that ${ }^{\dagger} \mathfrak{F}={ }^{\ddagger} \mathfrak{F}=\mathfrak{F}\left({ }^{*} \mathfrak{D}\right)$, and that the given log-link $\mathfrak{F}\left({ }^{*} \mathfrak{D}\right)=$ $\dagger \mathfrak{F} \stackrel{\mathfrak{l o g}}{\longrightarrow} \ddagger \mathfrak{F}=\mathfrak{F}\left({ }^{*} \mathfrak{D}\right)$ is the full log-link. Then there exists a functorial algorithm 
in the $\mathcal{D}$-prime-strip ${ }^{*} \mathfrak{D}$ for constructing a collection of topological subspaces - i.e., a collection of "coric holomorphic log-shells"

$$
\mathcal{I}_{* \mathfrak{D}} \stackrel{\text { def }}{=} \mathcal{I}_{\dagger \mathfrak{F}}
$$

of the collection $\Psi \frac{\mathrm{gp}}{\mathrm{cns}}\left({ }^{*} \mathfrak{D}\right)$, which may be naturally identified with $\Psi \frac{\mathrm{gp}}{\mathrm{cns}}\left({ }^{\ddagger} \mathfrak{F}\right)$, together with a collection of natural isomorphisms [cf. (viii); the fourth display of [IUTchII], Corollary 4.5, (ii)]

$$
\mathcal{I}_{* \mathfrak{D}} \stackrel{\sim}{\rightarrow} \mathcal{I}_{* \mathfrak{D}}
$$

— where we write ${ }^{*} \mathfrak{D}^{\vdash}$ for the $\mathcal{D}^{\vdash}$-prime-strip determined by ${ }^{*} \mathfrak{D}$.

(x) (Frobenius-picture) Let $\left\{{ }^{n} \mathfrak{F}\right\}_{n \in \mathbb{Z}}$ be a collection of distinct $\mathcal{F}$-primestrips [relative to the given initial $\Theta$-data - cf. [IUTchI], Definition 5.2, (i)] indexed by the integers. Write $\left\{{ }^{n} \mathfrak{D}\right\}_{n \in \mathbb{Z}}$ for the associated $\mathcal{D}$-prime-strips [cf. [IUTchI], Remark 5.2.1, (i)] and $\left\{{ }^{n} \mathfrak{D}^{\vdash}\right\}_{n \in \mathbb{Z}}$ for the associated $\mathcal{D}^{\vdash}$-prime-strips [cf. [IUTchI], Definition 4.1, (iv)]. Then the full log-links ${ }^{n} \mathfrak{F} \stackrel{\text { log }}{\longrightarrow} \quad(n+1) \mathfrak{F}$, for $n \in \mathbb{Z}$, give rise to an infinite chain

$$
\ldots \quad \stackrel{\log }{\longrightarrow}(n-1) \mathfrak{F} \stackrel{\log }{\longrightarrow} n \mathfrak{F} \stackrel{\log }{\longrightarrow}(n+1) \mathfrak{F} \stackrel{\mathfrak{l o g}}{\longrightarrow} \ldots
$$

of log-linked $\mathcal{F}$-prime-strips which induces chains of full poly-isomorphisms

$$
\ldots \stackrel{\sim}{\rightarrow} n \mathfrak{D} \stackrel{\sim}{\rightarrow}(n+1) \mathfrak{D} \stackrel{\sim}{\rightarrow} \ldots \quad \text { and } \quad \ldots \stackrel{\sim}{\rightarrow}^{n} \mathfrak{D}^{\vdash} \stackrel{\sim}{\rightarrow}^{(n+1)} \mathfrak{D}^{\vdash} \stackrel{\sim}{\rightarrow} \ldots
$$

on the associated $\mathcal{D}$ - and $\mathcal{D}^{\vdash}$-prime-strips. These chains may be represented symbolically as an oriented graph $\vec{\Gamma}$ [cf. [AbsTopIII], §0]

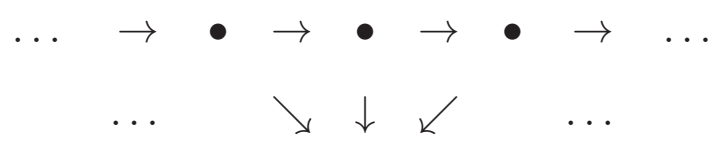

○

- i.e., where the horizontal arrows correspond to the " $\stackrel{\text { log }}{\longrightarrow}$ 's"; the "• 's" correspond to the "n F"; the "o" corresponds to the "n $\mathfrak{D}$ ", identified up to isomorphism; the vertical/diagonal arrows correspond to the Kummer isomorphisms of (iv). This oriented graph $\vec{\Gamma}$ admits a natural action by $\mathbb{Z}$ [cf. [AbsTopIII], Corollary 5.5, (v)] - i.e., a translation symmetry - that fixes the "core" o, but it does not admit arbitrary permutation symmetries. For instance, $\vec{\Gamma}$ does not admit an automorphism that switches two adjacent vertices, but leaves the remaining vertices fixed.

Proof. The various assertions of Proposition 1.2 follow immediately from the definitions and the references quoted in the statements of these assertions.

Remark 1.2.1. Suppose that we are in the situation of Definition 1.1, (ii). Then at the level of metrics - i.e., which give rise to angular log-volumes as in 
Proposition 1.2, (iii) — we suppose that $\Psi_{\uparrow \mathcal{F}_{\underline{v}}}^{\mathrm{gp}} / \Psi_{\uparrow \mathcal{F}_{\underline{v}}}^{\boldsymbol{\mu}_{N}}$ is equipped with the metric obtained by descending the metric of $\Psi_{\dagger \mathcal{F}_{\underline{v}}}^{\text {gp }}$, but we regard the object

$\Psi_{\dagger \mathcal{F}_{\underline{v}}}^{\mathrm{gp}} / \Psi_{\dagger \mathcal{F}_{\underline{v}}}^{\boldsymbol{\mu}_{N}}\left[\operatorname{or} \Psi_{\dagger \mathcal{F}_{\underline{v}}}^{\times} / \Psi_{\dagger \mathcal{F}_{\underline{v}}}^{\boldsymbol{\mu}_{N}}\right]$ as being equipped with a "weight $N$ "

- i.e., which has the effect of ensuring that the log-volume of $\Psi_{\dagger_{\mathcal{F}_{\underline{v}}}}^{\times} / \Psi_{\uparrow_{\mathcal{F}_{\underline{v}}}}^{\boldsymbol{\mu}_{N}}$ is equal to that of $\Psi_{\dagger \mathcal{F}_{v}}^{\times}$. That is to say, this convention concerning "weights" ensures that working with $\bar{\Psi}_{\uparrow \mathcal{F}_{\underline{v}}}^{\text {gp }} / \Psi_{\uparrow \mathcal{F}_{\underline{v}}}^{\mu_{N}}$ does not have any effect on various computations of logvolume.

Remark 1.2.2. Before proceeding, we pause to consider the significance of the various properties discussed in Proposition 1.2, (v). For simplicity, we suppose that "† $\mathfrak{F}$ " is the $\mathcal{F}$-prime-strip that arises from the data constructed in [IUTchI], Examples 3.2, (iii); 3.3, (i); 3.4, (i) [cf. [IUTchI], Definition 5.2, (i)].

(i) Suppose that $\underline{v} \in \underline{\mathbb{V}}^{\text {non }}$. Thus, $K_{\underline{v}}$ [cf. the notation of [IUTchI], Definition $3.1,(\mathrm{e})]$ is a mixed-characteristic nonarchimedean local field. Write $k \stackrel{\text { def }}{=} K_{\underline{v}}$, $\mathcal{O}_{k} \subseteq k$ for the ring of integers of $k, \mathcal{O}_{k}^{\times} \subseteq \mathcal{O}_{k}$ for the group of units, and $\log _{k}$ : $\mathcal{O}_{k}^{\times} \rightarrow k$ for the $p_{\underline{v}}$-adic logarithm. Then, at a more concrete level - i.e., relative to the notation of the present discussion - the log-shell " $\mathcal{I}_{\dagger \mathcal{F}_{\underline{v}}}$ " corresponds to the submodule

$$
\mathcal{I}_{k} \stackrel{\text { def }}{=}\left(p_{\underline{v}}^{*}\right)^{-1} \cdot \log _{k}\left(\mathcal{O}_{k}^{\times}\right) \subseteq k
$$

— where $p_{\underline{v}}^{*}=p_{\underline{v}}$ if $p_{\underline{v}}$ is odd, $p_{\underline{v}}^{*}=p_{\underline{v}}^{2}$ if $p_{\underline{v}}$ is even - while the properties $\left(\mathrm{b}_{\mathrm{non}}\right)$, $\left(\mathrm{c}_{\mathrm{non}}\right)$ of Proposition $1.2,(\mathrm{v})$, correspond, respectively, to the evident inclusions

$$
\mathcal{O}_{k}^{\triangleright} \stackrel{\text { def }}{=} \mathcal{O}_{k} \backslash\{0\} \subseteq \mathcal{O}_{k} \subseteq \mathcal{I}_{k} ; \quad \log _{k}\left(\mathcal{O}_{k}^{\times}\right) \subseteq \mathcal{I}_{k}
$$

of subsets of $k$.

(ii) Suppose that $\underline{v} \in \underline{\mathbb{V}}^{\text {arc }}$. Thus, $K_{\underline{v}}$ [cf. the notation of [IUTchI], Definition 3.1 , (e)] is a complex archimedean field. Write $k \stackrel{\text { def }}{=} K_{\underline{v}}, \mathcal{O}_{k} \subseteq k$ for the subset of elements of absolute value $\leq 1, \mathcal{O}_{k}^{\times} \subseteq \mathcal{O}_{k}$ for the group of elements of absolute value $=1$, and $\exp _{k}: k \rightarrow k^{\times}$for the exponential map. Then, at a more concrete level — i.e., relative to the notation of the present discussion — the log-shell " $\mathcal{I}_{\dagger \mathcal{F}_{\underline{\underline{v}}}}$ " corresponds to the subset

$$
\mathcal{I}_{k} \stackrel{\text { def }}{=}\{a \in k|| a \mid \leq \pi\} \subseteq k
$$

of elements of absolute value $\leq \pi$, while the properties $\left(b_{\text {arc }}\right),\left(c_{\text {arc }}\right)$ of Proposition $1.2,(\mathrm{v})$, correspond, respectively, to the evident inclusions

$$
\mathcal{O}_{k}^{\triangleright} \stackrel{\text { def }}{=} \mathcal{O}_{k} \backslash\{0\} \subseteq \mathcal{O}_{k} \subseteq \mathcal{I}_{k} ; \quad \mathcal{O}_{k}^{\times} \subseteq \exp _{k}\left(\mathcal{I}_{k}\right)
$$

- where we note the slightly different roles played, in the archimedean [cf. the present (ii)] and nonarchimedean [cf. (i)] cases, by the exponential and logarithmic functions, respectively [cf. [AbsTopIII], Remark 4.5.2]. 
(iii) The diagram represented by the oriented graph $\vec{\Gamma}$ of Proposition $1.2,(\mathrm{x})$, is, of course, far from commutative [cf. Proposition 1.2, (iv)]! Ultimately, however, [cf. the discussion of Remark 1.4.1, (ii), below] we shall be interested in

(a) constructing invariants with respect to the $\mathbb{Z}$-action on $\vec{\Gamma}$ - i.e., in effect, constructing objects via functorial algorithms in the coric $\mathcal{D}$-prime-strips "n $\mathfrak{D} "-$

while, at the same time,

(b) relating the corically constructed objects of (a) to the non-coric " $n \mathfrak{F}$ " via the various Kummer isomorphisms of Proposition 1.2, (iv).

That is to say, from the point of view of (a), (b), the content of the inclusions discussed in (i) and (ii) above may be interpreted, at $\underline{v} \in \underline{\mathbb{V}}^{\text {non }}$, as follows:

the coric holomorphic log-shells of Proposition 1.2, (ix), contain not only the images, via the Kummer isomorphisms [i.e., the vertical/diagonal arrows of $\vec{\Gamma}]$, of the various " $\mathcal{O}^{\triangleright}$ " at $\underline{v} \in \underline{\mathbb{V}}^{\text {non }}$, but also the images, via the composite of the Kummer isomorphisms with the various iterates of the log-link [i.e., the horizontal arrows of $\vec{\Gamma}$ ], of the portions of the various "O $\mathrm{O}^{\triangleright "}$ at $\underline{v} \in \underline{\mathbb{V}}^{\text {non }}$ on which these iterates are defined.

An analogous statement in the case of $\underline{v} \in \underline{\mathbb{V}}^{\text {arc }}$ may be formulated by adjusting the wording appropriately so as accommodate the latter portion of this statement, which corresponds to a certain surjectivity - we leave the routine details to the reader. Thus, although the diagram [corresponding to] $\vec{\Gamma}$ fails to be commutative,

the coric holomorphic log-shells involved exhibit a sort of "upper semicommutativity" with respect to containing/surjecting onto the various images arising from composites of arrows in $\vec{\Gamma}$.

(iv) Note that although the diagram $\vec{\Gamma}$ admits a natural "upper semi-commutativity" interpretation as discussed in (iii) above, it fails to admit a corresponding "lower semi-commutativity" interpretation. Indeed, such a "lower semi-commutativity" interpretation would amount to the existence of some sort of collection of portions of the various " $\mathrm{O}^{\triangleright}$ 's" involved [cf. the discussion of (i), (ii) above] - i.e., a sort of "core" - that are mapped to one another isomorphically by the various maps " $\log _{k} " /$ " $\exp _{k}$ " [cf. the discussion of (i), (ii) above] in a fashion that is compatible with the various Kummer isomorphisms that appear in the diagram $\vec{\Gamma}$. On the other hand, it is difficult to see how to construct such a collection of portions of the various " $\mathcal{O}^{\triangleright}$ ' $s$ " involved.

(v) Proposition 1.2, (iii), may be interpreted in the spirit of the discussion of (iii) above. That is to say, although the diagram corresponding to $\vec{\Gamma}$ fails to be commutative, it is nevertheless "commutative with respect to log-volumes", in the sense discussed in Proposition 1.2, (iii). This "commutativity with respect to log-volumes" allows one to work with log-volumes in a fashion that is consistent 
with all composites of the various arrows of $\vec{\Gamma}$. Log-volumes will play an important role in the theory of $\S 3$, below, as a sort of mono-analytic version of the notion of the degree of a global arithmetic line bundle [cf. the theory of [AbsTopIII], §5].

(vi) As discussed in [AbsTopIII], $\S \mathrm{I} 3$, the log-links of $\vec{\Gamma}$ may be thought of as a sort of "juggling of $\boxplus$, $\nabla$ " [i.e., of the two combinatorial dimensions of the ring structure constituted by addition and multiplication]. The "arithmetic holomorphic structure" constituted by the coric $\mathcal{D}$-prime-strips is immune to this juggling, and hence may be thought as representing a sort of quotient of the horizontal arrow portion of $\vec{\Gamma}$ by the action of $\mathbb{Z}$ [cf. (iii), (a)] — i.e., at the level of abstract oriented graphs, as a sort of "oriented copy of $\mathbb{S}^{1}$ ". That is to say, the horizontal arrow portion of $\vec{\Gamma}$ may be thought of as a sort of "unraveling" of this "oriented copy of $\mathbb{S}^{1}$ ", which is subject to the "juggling of $\boxplus, \otimes$ " constituted by the $\mathbb{Z}$-action. Here, it is useful to recall that

(a) the Frobenius-like structures constituted by the monoids that appear in the horizontal arrow portion of $\vec{\Gamma}$ play the crucial role in the theory of the present series of papers of allowing one to construct such "nonring/scheme-theoretic filters" as the $\Theta$-link [cf. the discussion of [IUTchII], Remark 3.6.2, (ii)].

By contrast,

(b) the étale-like structures constituted by the coric $\mathcal{D}$-prime-strips play the crucial role in the theory of the present series of papers of allowing one to construct objects that are capable of "functorially permeating" such non-ring/scheme-theoretic filters as the $\Theta$-link [cf. the discussion of [IUTchII], Remark 3.6.2, (ii)].

Finally, in order to relate the theory of (a) to the theory of (b), one must avail oneself of Kummer theory [cf. (iii), (b), above].

\begin{tabular}{|c|c|}
\hline $\begin{array}{c}\text { mono-anabelian coric } \\
\text { étale-like structures }\end{array}$ & invariant differential \\
& $d \theta$ on $\mathbb{S}^{1}$ \\
\hline post-anabelian & coordinate functions \\
Frobenius-like structures & $\int_{\bullet} d \theta$ on $\vec{\Gamma}$ \\
\hline
\end{tabular}

Fig. 1.1: Analogy with the differential geometry of $\mathbb{S}^{1}$

(vii) From the point of view of the discussion in (vi) above of the "oriented copy of $\mathbb{S}^{1}$ " obtained by forming the quotient of the horizontal arrow portion of $\vec{\Gamma}$ by $\mathbb{Z}$, one may think of the coric étale-like structures of Proposition 1.2, (i) - as well as the various objects constructed from these coric étale-like structures via the various mono-anabelian algorithms discussed in [AbsTopIII] — as corresponding to 
the "canonical invariant differential $d \theta$ " on $\mathbb{S}^{1}[$ which is, in particular, invariant with respect to the action of $\mathbb{Z} !]$. On the other hand, the various post-anabelian Frobenius-like structures obtained by forgetting the mono-anabelian algorithms applied to construct these objects - cf., e.g.., the " $\Psi_{\text {cns }}\left({ }^{\dagger} \mathfrak{F}\right)$ " that appear in the Kummer isomorphisms of Proposition 1.2, (iv) - may be thought of as coordinate functions on the horizontal arrow portion of $\vec{\Gamma}$ [which are not invariant with respect to the action of $\mathbb{Z}$ !] of the form " $\int_{\bullet} d \theta$ " obtained by integrating the invariant differential $d \theta$ along various paths of $\vec{\Gamma}$ that emanate from some fixed vertex "॰" of $\vec{\Gamma}$. This point of view is summarized in Fig. 1.1 above. Finally, we observe that this point of view is reminiscent of the discussion of [AbsTopIII], §I5, concerning the analogy between the theory of [AbsTopIII] and the construction of canonical coordinates via integration of Frobenius-invariant differentials in the classical $p$-adic theory.

\section{Remark 1.2.3.}

(i) Observe that, relative to the notation of Remark 1.2.2, (i), any multiplicative indeterminacy with respect to the action on $\mathcal{O}_{k}^{\triangleright}$ of some subgroup $H \subseteq \mathcal{O}_{k}^{\times}$at some "•" of the diagram $\vec{\Gamma}$ gives rise to an additive indeterminacy with respect to the action of $\log _{k}(H)$ on the copy of " $\mathcal{O}_{k}$ " that corresponds to the subsequent "•" of the diagram $\vec{\Gamma}$. In particular, if $H$ consists of roots of unity, then $\log _{k}(H)=\{0\}$, so the resulting additive indeterminacy ceases to exist. This observation will play a crucial role in the theory of $\S 3$, below, when it is applied in the context of the constant multiple rigidity properties constituted by the canonical splittings of theta and Gaussian monoids discussed in [IUTchII], Proposition 3.3, (i); [IUTchII], Corollary 3.5, (iii) [cf. also [IUTchII], Corollary 1.12, (ii); the discussion of [IUTchII], Remark 1.12.2, (iv)].

(ii) In the theory of $\S 3$, below, we shall consider global arithmetic line bundles. This amounts, in effect, to considering multiplicative translates by $f \in F_{\bmod }^{\times}$ of the product of the various " $\mathcal{O}_{k}^{\times}$" of Remark 1.2.2, (i), (ii), as $\underline{v}$ ranges over the elements of $\mathbb{V}$. Such translates are disjoint from one another, except in the case where $f$ is a unit at all $\underline{v} \in \underline{\mathbb{V}}$. By elementary algebraic number theory [cf., e.g., [Lang], p. 144, the proof of Theorem 5], this corresponds precisely to the case where $f$ is a root of unity. In particular, to consider quotients by this multiplicative action by $F_{\text {mod }}^{\times}$at one "•" of the diagram $\vec{\Gamma}$ [where we allow $\underline{v}$ to range over the elements of $\underline{\mathbb{V}}]$ gives rise to an additive indeterminacy by "logarithms of roots of unity" at the subsequent "•" of the diagram $\vec{\Gamma}$. In particular, at $\underline{v} \in \underline{\mathbb{V}}^{\text {non }}$, the resulting additive indeterminacy ceases to exist [cf. the discussion of (i); Definition 1.1, (iv)]; at $\underline{v} \in \underline{\mathbb{V}}^{\text {arc }}$, the resulting indeterminacy corresponds to considering certain quotients of the copies of " $\mathcal{O}_{k}^{\times}$" — i.e., of " $\mathbb{S}^{1}$ " — that appear by some finite group [cf. the discussion of Definition 1.1, (ii)]. These observations will be of use in the development of the theory of $\S 3$, below.

\section{Remark 1.2.4.}

(i) At this point, we pause to recall the important observation that the log-link is incompatible with the ring structures of $\Psi_{\dagger \mathcal{F}_{\underline{v}}}^{\mathrm{gp}}$ and $\Psi_{\mathfrak{l o g}\left({ }^{\dagger} \mathcal{F}_{\underline{v}}\right)}[$ cf. the notation 
of Proposition 1.2, (ii)], in the sense that it does not arise from a ring homomorphism between these two rings. The barrier constituted by this incompatibility between the ring structures on either side of the log-link is precisely what is referred to as the "log-wall" in the theory of [AbsTopIII] [cf. the discussion of [AbsTopIII], §I4]. This incompatibility with the respective ring structures implies that it is not possible, a priori, to transport objects whose structure depends on these ring structures via the log-link by invoking the principle of "transport of structure". From the point of view of the theory of the present series of papers, this means, in particular, that

the log-wall is incompatible with conventional scheme-theoretic basepoints, which are defined by means of geometric points [i.e., ring homomorphisms of a certain type]

— cf. the discussion of [IUTchII], Remark 3.6.3, (i); [AbsTopIII], Remark 3.7.7, (i). In this context, it is useful to recall that étale fundamental groups - i.e., Galois groups - are defined as certain automorphism groups of fields/rings; in particular, the definition of such a Galois group "as a certain automorphism group of some ring structure" is incompatible, in a quite essential way, with the log-wall. In a similar vein, Kummer theory, which depends on the multiplicative structure of the ring under consideration, is also incompatible, in a quite essential way, with the log-wall [cf. Proposition 1.2, (iv)]. That is to say, in the context of the log-link,

the only structure of interest that is manifestly compatible with the loglink [cf. Proposition 1.2, (i), (ii)] is the associated $\mathcal{D}$-prime-strip

— i.e., the abstract topological groups [isomorphic to " $\Pi_{\underline{v}}$ " - cf. the notation of [IUTchI], Definition 3.1, (e), (f)] at $\underline{v} \in \underline{\mathbb{V}}^{\text {non }}$ and abstract Aut-holomorphic spaces [isomorphic to " $\mathbb{U}_{\underline{v}}$ " - cf. the notation of [IUTchII], Proposition 4.3] at $\underline{v} \in \underline{\mathbb{V}}^{\text {arc }}$. Indeed, this observation is precisely the starting point of the theory of [AbsTopIII] [cf. the discussion of [AbsTopIII], §I1, §I4].

(ii) Other important examples of structures which are incompatible with the $\mathfrak{l o g}$-wall include

(a) the additive structure on the image of the Kummer map [cf. the discussion of [AbsTopIII], Remark 3.7.5];

(b) in the "birational" situation - i.e., where one replaces " $\Pi_{\underline{v}}$ " by the absolute Galois group $\Pi_{\underline{v}}^{\text {birat }}$ of the function field of the affine curve that gave rise to $\Pi_{\underline{v}}$ — the datum of the collection of closed points that determines the affine curve [cf. [AbsTopIII], Remark 3.7.7, (ii)].

Note, for instance in the case of (b), that one may think of the additional datum under consideration as consisting of the natural outer surjection $\Pi_{\underline{v}}^{\text {birat }} \rightarrow \Pi_{\underline{v}}$ that arises from the scheme-theoretic morphism from the spectrum of the function field to the given affine curve. On the other hand, just as in the case of the discussion of scheme-theoretic basepoints in (i), the construction of such an object $\Pi_{\underline{v}}^{\text {birat }} \rightarrow \Pi_{\underline{v}}$ whose structure depends, in an essential way, on the scheme [i.e., ring!] structures 
involved necessarily fails to be compatible with the log-link [cf. the discussion of [AbsTopIII], Remark 3.7.7, (ii)].

(iii) One way to understand the incompatibility discussed in (ii), (b), is as follows. Write $\Delta_{\underline{v}}^{\text {birat }}, \Delta_{\underline{v}}$ for the respective kernels of the natural surjections $\Pi_{\underline{v}}^{\text {birat }} \rightarrow G_{\underline{v}}, \Pi_{\underline{v}} \rightarrow G_{\underline{v}}$. Then if one forgets about the scheme-theoretic basepoints discussed in (i), $G_{\underline{v}}, \Delta_{\underline{v}}^{\text {birat }}$, and $\Delta_{\underline{v}}$ may be understood on both sides of the log-wall as "some topological group", and each of the topological groups $\Delta_{v}^{\text {birat }}$, $\Delta_{\underline{v}}$ may be understood on both sides of the log-wall as being equipped with "some outer $G_{\underline{v}}$-action" - cf. the two diagonal arrows of Fig. 1.2 below. On the other hand, the datum of a particular outer surjection $\Delta_{\underline{v}}^{\text {birat }} \rightarrow \Delta_{\underline{v}}$ [cf. the dotted line in Fig. 1.2] relating these two diagonal arrows - which depends, in an essential way, on the scheme [i.e., ring] structures involved! - necessarily fails to be compatible with the log-link [cf. the discussion of [AbsTopIII], Remark 3.7.7, (ii)]. This issue of "triangular compatibility between independent indeterminacies" is formally reminiscent of the issue of compatibility of outer homomorphisms discussed in [IUTchI], Remark 4.5.1, (i) [cf. also [IUTchII], Remark 2.5.2, (ii)].

indep.

bp. indet.

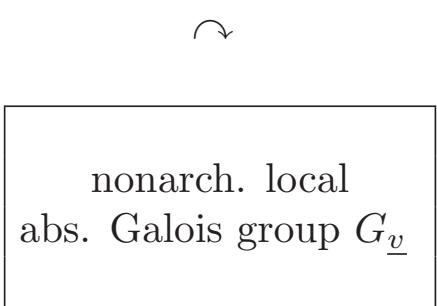

indep.

bp. indet.

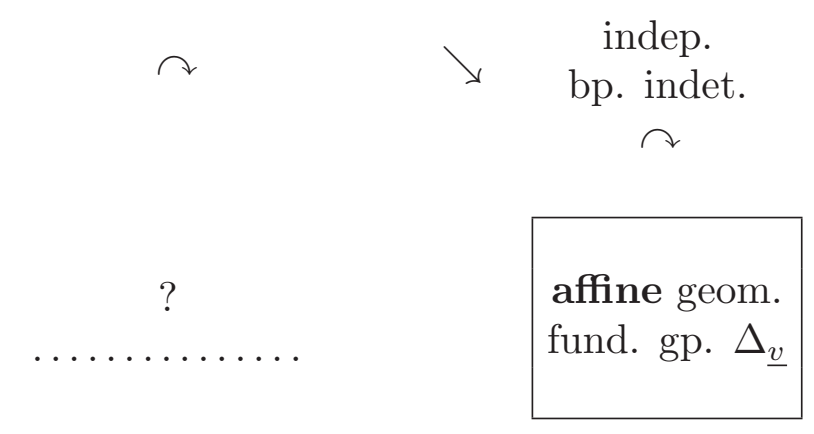

Fig. 1.2: Independent basepoint indeterminacies obstruct relationship between birational and affine geometric fundamental groups

\section{Proposition 1.3. (log-links Between $\Theta^{ \pm \text {ell }}$ NF-Hodge Theaters) Let}

$$
{ }^{\dagger} \mathcal{H} \mathcal{T}^{\Theta^{ \pm \mathrm{ell}} \mathrm{NF}} ; \quad \ddagger \mathcal{H} \mathcal{T}^{\Theta^{ \pm \mathrm{ell}} \mathrm{NF}}
$$

be $\Theta^{ \pm e l l} \mathbf{N F}-H o d g e$ theaters [relative to the given initial $\Theta$-data] - cf. [IUTchI], Definition 6.13, (i). Write ${ }^{\dagger} \mathcal{H} \mathcal{T}^{\mathcal{D}-\Theta^{ \pm \mathrm{ell}} \mathrm{NF}},{ }_{\mathcal{H}} \mathcal{T}^{\mathcal{D}-\Theta^{ \pm \mathrm{ell}} \mathrm{NF}}$ for the associated $\mathcal{D}$ $\Theta^{ \pm \text {ell }}$ NF-Hodge theaters - cf. [IUTchI], Definition 6.13, (ii). Then: 
(i) (Construction of the log-Link) Fix an isomorphism

$$
\Xi:{ }^{\dagger} \mathcal{H} \mathcal{T}^{\mathcal{D}-\Theta^{ \pm \mathrm{ell}} \mathrm{NF}} \stackrel{\sim}{\rightarrow} \ddagger \mathcal{H} \mathcal{T}^{\mathcal{D}-\Theta^{ \pm \mathrm{ell}} \mathrm{NF}}
$$

of $\mathcal{D}-\Theta^{ \pm \text {ell }} N F$-Hodge theaters. Let ${ }^{\dagger} \mathfrak{F}_{\square}$ be one of the $\mathcal{F}$-prime-strips that appear in the $\Theta$ - and $\Theta^{ \pm}$-bridges that constitute ${ }^{\dagger} \mathcal{H} \mathcal{T}^{\Theta^{ \pm \mathrm{ell}} \mathrm{NF}}-$ i.e., either one of the $\mathcal{F}$-prime-strips

$$
{ }^{\dagger} \mathfrak{F}_{>}, \quad{ }^{\mathfrak{F}_{\succ}}
$$

or one of the constituent $\mathcal{F}$-prime-strips of the capsules

$$
\dagger \mathfrak{F}_{J}, \quad \mathfrak{F}_{T}
$$

[cf. [IUTchI], Definition 5.5, (ii); [IUTchI], Definition 6.11, (i)]. Write ${ }^{\ddagger} \mathfrak{F}_{\square}$ for the corresponding $\mathcal{F}$-prime-strip of $\ddagger \mathcal{H} \mathcal{T}^{\Theta^{ \pm \mathrm{ell}} \mathrm{NF}}$. Then the poly-isomorphism determined by $\Xi$ between the $\mathcal{D}$-prime-strips associated to ${ }^{\dagger} \mathfrak{F}_{\square},{ }^{\ddagger} \mathfrak{F}_{\square}$ uniquely determines a poly-isomorphism $\log \left({ }^{\dagger} \mathfrak{F}_{\square}\right) \stackrel{\sim}{\rightarrow}{ }^{\ddagger} \mathfrak{F}_{\square}$ [cf. Definition 1.1, (iii); [IUTchI], Corollary 5.3, (ii)], hence a log-link ${ }^{\dagger} \mathfrak{F}_{\square} \stackrel{\mathfrak{l o g}}{\longrightarrow} \ddagger^{\ddagger} \mathfrak{F}_{\square}[c f$. Definition 1.1, (iii)]. We shall denote by

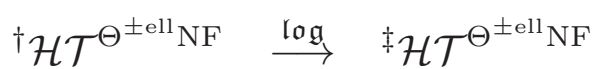

and refer to as a log-link from ${ }^{\dagger} \mathcal{H} \mathcal{T}^{\Theta^{ \pm \mathrm{ell}} \mathrm{NF}}$ to ${ }^{\ddagger} \mathcal{H} \mathcal{T}^{\Theta^{ \pm \mathrm{ell}} \mathrm{NF}}$ the collection of data consisting of $\Xi$, together with the collection of log-links ${ }^{\dagger} \mathfrak{F}_{\square} \stackrel{\log }{\longrightarrow} \quad \ddagger \mathfrak{F}_{\square}$, as “ $\square$ " ranges over all possibilities for the $\mathcal{F}$-prime-strips in question. When $\Xi$ is replaced by a poly-isomorphism ${ }^{\dagger} \mathcal{H} \mathcal{T}^{\mathcal{D}-\Theta^{ \pm \mathrm{ell}} \mathrm{NF}} \stackrel{\sim}{\rightarrow} \ddagger \mathcal{H} \mathcal{T}^{\mathcal{D}-\Theta^{ \pm \mathrm{ell}} \mathrm{NF}}$, we shall also refer to the resulting collection of log-links [i.e., corresponding to each constituent isomorphism of the poly-isomorphism $\Xi$ ] as a log-link from ${ }^{\dagger} \mathcal{H} \mathcal{T}^{\Theta^{ \pm \mathrm{ell}} \mathrm{NF}}$ to ${ }^{\ddagger} \mathcal{H} \mathcal{T}^{\Theta^{ \pm \mathrm{ell}} \mathrm{NF}}$. When $\Xi$ is the full poly-isomorphism, we shall refer to the resulting log-link as the full log-link. When ${ }^{\dagger} \mathcal{H} \mathcal{T}^{\Theta^{ \pm \mathrm{ell}} \mathrm{NF}}={ }^{\ddagger} \mathcal{H} \mathcal{T}^{\Theta^{ \pm \mathrm{ell}} \mathrm{NF}}$, and $\Xi$ is the identity, then we shall refer to the resulting log-link as the tautological log-link.

(ii) (Coricity) Any log-link ${ }^{\dagger} \mathcal{H} \mathcal{T}^{\Theta^{ \pm \mathrm{ell}} \mathrm{NF}} \stackrel{\log }{\longrightarrow} \ddagger \mathcal{H} \mathcal{T}^{\Theta^{ \pm \mathrm{ell}} \mathrm{NF}}$ induces [and may be thought of as "lying over"] a [poly-]isomorphism

$$
\dagger \mathcal{H} \mathcal{T}^{\mathcal{D}-\Theta^{ \pm \mathrm{ell}} \mathrm{NF}} \stackrel{\sim}{\rightarrow} \ddagger \mathcal{H} \mathcal{T}^{\mathcal{D}-\Theta^{ \pm \mathrm{ell}} \mathrm{NF}}
$$

of $\mathcal{D}-\Theta^{ \pm \mathrm{ell}} N F$-Hodge theaters [and indeed coincides with the log-link constructed in (i) from this [poly-]isomorphism of $\mathcal{D}-\Theta^{ \pm \mathrm{ell}} \mathrm{NF}$-Hodge theaters].

(iii) (Further Properties of the log-Link) In the notation of (i), any loglink $^{\dagger} \mathcal{H} \mathcal{T}^{\Theta^{ \pm \mathrm{ell}} \mathrm{NF}} \stackrel{\mathfrak{l o g}}{\longrightarrow} \ddagger \mathcal{H} \mathcal{T}^{\Theta^{ \pm \mathrm{ell}} \mathrm{NF}}$ satisfies, for each $\mathcal{F}$-prime-strip ${ }^{\dagger} \mathfrak{F}_{\square}$, properties corresponding to the properties of Proposition 1.2, (ii), (iii), (iv), (v), (vi), (vii), (viii), (ix) i.e., concerning simultaneous compatibility with ring structures and log-volumes, Kummer theory, and log-shells.

(iv) (Frobenius-picture) Let $\left\{{ }^{n} \mathcal{H} \mathcal{T}^{\Theta^{ \pm e l l} \mathrm{NF}}\right\}_{n \in \mathbb{Z}}$ be a collection of distinct $\Theta^{ \pm \text {ell }} \mathbf{N F - H o d g e}$ theaters [relative to the given initial $\Theta$-data] indexed by the integers. Write $\left\{{ }^{n} \mathcal{H} \mathcal{T}^{\mathcal{D}-\Theta^{ \pm e l l} \mathrm{NF}}\right\}_{n \in \mathbb{Z}}$ for the associated $\mathcal{D}-\Theta^{ \pm \mathrm{ell}} N F$-Hodge theaters. 
Then the full log-links ${ }^{n} \mathcal{H} \mathcal{T}^{\Theta^{ \pm \mathrm{ell}} \mathrm{NF}} \stackrel{\mathrm{log}}{\longrightarrow} \quad{ }^{(n+1)} \mathcal{H} \mathcal{T}^{\Theta^{ \pm \mathrm{ell}} \mathrm{NF}}$, for $n \in \mathbb{Z}$, give rise to an infinite chain

$$
\ldots \stackrel{\text { log }}{\longrightarrow}(n-1) \mathcal{H} \mathcal{T}^{\Theta^{ \pm \mathrm{ell}} \mathrm{NF}} \stackrel{\log }{\longrightarrow}{ }^{n} \mathcal{H} \mathcal{T}^{\Theta^{ \pm \mathrm{ell}} \mathrm{NF}} \stackrel{\mathrm{log}}{\longrightarrow} \quad{ }^{(n+1)} \mathcal{H} \mathcal{T}^{\Theta^{ \pm \mathrm{ell}} \mathrm{NF}} \stackrel{\text { log }}{\longrightarrow} \quad \ldots
$$

of log-linked $\mathcal{F}$-prime-strips which induces a chain of full poly-isomorphisms

$$
\ldots \stackrel{\sim}{\rightarrow}{ }^{n} \mathcal{H} \mathcal{T}^{\mathcal{D}-\Theta^{ \pm \text {ell }} \mathrm{NF}} \stackrel{\sim}{\rightarrow}{ }^{(n+1)} \mathcal{H} \mathcal{T}^{\mathcal{D}-\Theta^{ \pm \text {ell }} \mathrm{NF}} \stackrel{\sim}{\rightarrow} \ldots
$$

on the associated $\mathcal{D}-\Theta^{ \pm e l l} N F-H o d g e$ theaters. These chains may be represented symbolically as an oriented graph $\vec{\Gamma}$ [cf. [AbsTopIII], §O]

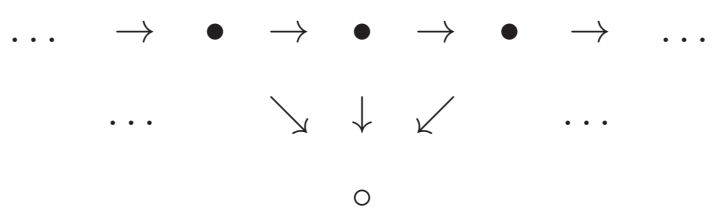

- i.e., where the horizontal arrows correspond to the " $\stackrel{\text { log }}{\longrightarrow}$ 's"; the "•'s" corre-

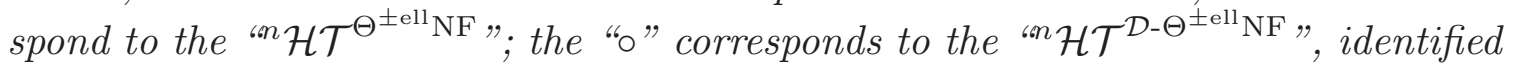
up to isomorphism; the vertical/diagonal arrows correspond to the Kummer isomorphisms implicit in the statement of (iii). This oriented graph $\vec{\Gamma}$ admits a natural action by $\mathbb{Z}$ [cf. [AbsTopIII], Corollary 5.5, (v)] - i.e., a translation symmetry - that fixes the "core" o, but it does not admit arbitrary permutation symmetries. For instance, $\vec{\Gamma}$ does not admit an automorphism that switches two adjacent vertices, but leaves the remaining vertices fixed.

Proof. The various assertions of Proposition 1.3 follow immediately from the definitions and the references quoted in the statements of these assertions.

Remark 1.3.1. Note that in Proposition 1.3, (i), it was necessary to carry out the given construction of the $\mathfrak{l o g}$-link first for a single $\Xi$ [i.e., as opposed to a poly-isomorphism $\Xi]$, in order to maintain compatibility with the crucial " \pm synchronization" [cf. [IUTchI], Remark 6.12.4, (iii); [IUTchII], Remark 4.5.3, (iii)] inherent in the structure of a $\Theta^{ \pm \text {ell }}$-Hodge theater.

Remark 1.3.2. In the construction of Proposition 1.3, (i), the constituent $\mathcal{F}$ prime-strips ${ }^{\dagger} \mathfrak{F}_{t}$, for $t \in T$, of the capsule ${ }^{\dagger} \mathfrak{F}_{T}$ are considered without regard to the $\mathbb{F}_{l}^{\rtimes \pm}$-symmetries discussed in [IUTchII], Corollary 4.6, (iii). On the other hand, one verifies immediately that the $\mathfrak{l o g}$-links associated, in the construction of Proposition 1.3, (i), to these $\mathcal{F}$-prime-strips ${ }^{\dagger} \mathfrak{F}_{t}$, for $t \in T$ - i.e., more precisely, associated to the labeled collections of monoids $\Psi_{\mathrm{cns}}\left({ }^{\dagger} \mathfrak{F}_{\succ}\right)_{t}$ of [IUTchII], Corollary 4.6, (iii) - are in fact compatible with the $\mathbb{F}_{l}^{\rtimes \pm}$-symmetrizing isomorphisms discussed in [IUTchII], Corollary 4.6, (iii), hence also with the conjugate synchronization determined by these $\mathbb{F}_{l}^{\rtimes \pm}$-symmetrizing isomorphisms - cf. the discussion of Step (vi) of the proof of Corollary 3.12 of $\S 3$ below. We leave the routine details to the reader. 


\section{Remark 1.3.3.}

(i) In the context of Proposition 1.3, it is of interest to observe that the relationship between the various Frobenioid-theoretic [i.e., Frobenius-like!] portions of the $\Theta^{ \pm \text {ell }}$ NF-Hodge theaters in the domain and codomain the log-link of Proposition $1.3,(\mathrm{i})$,

does not include any data that is incompatible, relative to the relevant Kummer isomorphisms, with the log-wall

— cf. the discussion of Remark 1.2.4, (ii), (a), (b); Remark 1.2.4, (iii). This follows immediately from the fact [cf. Remarks 1.3.1, 1.3.2; [IUTchI], Corollary 5.3, (i), (ii), (iv); [IUTchI], Corollary 5.6, (i), (ii), (iii)] that these Frobenioid-theoretic portions of the $\Theta^{ \pm \text {ell }}$ NF-Hodge theaters under consideration are completely [i.e., fully faithfully! controlled [cf. the discussion of (ii) below for more details], via functorial algorithms, by the corresponding étale-like structures, i.e., structures that appear in the associated $\mathcal{D}-\Theta^{ \pm \text {ell }} \mathrm{NF}$-Hodge theaters, which satisfy the crucial coricity property of Proposition 1.3, (ii).

(ii) In the context of (i), it is of interest to recall that the global portion of the underlying $\Theta^{\text {ell }}$-bridges is defined [cf. [IUTchI], Definition 6.11, (ii)] in such a way that is does not contain any global Frobenioid-theoretic data! In particular, the issue discussed in (i) only concerns the Frobenioid-theoretic portions of the following:

(a) the various $\mathcal{F}$-prime-strips that appear;

(b) the underlying $\Theta$-Hodge theaters of the $\Theta^{ \pm \text {ell }} \mathrm{NF}$-Hodge theaters under consideration;

(c) the global portion of the underlying NF-bridges of the $\Theta^{ \pm \text {ell }}$ NF-Hodge theaters under consideration.

Here, the Frobenioid-theoretic data of (c) gives rise to independent basepoints with respect to the $\mathbb{F}_{l}^{*}$-symmetry [cf. [IUTchI], Corollary 5.6, (iii); [IUTchI], Remark 6.12.6, (iii); [IUTchII], Remark 4.7.6]. On the other hand, the independent basepoints that arise from the Frobenioid-theoretic data of (b), as well as of the portion of (a) that lies in the underlying $\Theta N F-H o d g e$ theater, do not cause any problems since this data is only subject to relationships defined by means of full poly-isomorphisms [cf. [IUTchI], Examples 4.3, 4.4]. That is to say, the $\mathcal{F}$-primestrips that lie in the underlying $\Theta^{ \pm \text {ell }}$-Hodge theater constitute the most delicate [i.e., relative to the issue of independent basepoints!] portion of the Frobenioidtheoretic data of a $\Theta^{ \pm \text {ell }} \mathrm{NF}-\mathrm{Hodge}$ theater. This delicacy revolves around the global synchronization of \pm -indeterminacies in the underlying $\Theta^{ \pm \text {ell }}$-Hodge theater [cf. [IUTchI], Remark 6.12.4, (iii); [IUTchII], Remark 4.5.3, (iii)]. On the other hand, this delicacy does not in fact cause any problems since the synchronizations of \pm -indeterminacies in the underlying $\Theta^{ \pm \text {ell }}$-Hodge theater are defined [not by means of scheme-theoretic relationships, but rather] by means of the structure of the underlying $\mathcal{D}-\Theta^{ \pm \text {ell }}$-Hodge theater, which satisfies the crucial coricity property of Proposition 1.3, (ii) [cf. the discussion of (i)]. 
The diagrams discussed in the following Definition 1.4 will play a central role in the theory of the present series of papers.

Definition 1.4. We maintain the notation of Proposition 1.3 [cf. also [IUTchII], Corollary 4.10, (iii)]. Let $\left\{{ }^{n, m} \mathcal{H} \mathcal{T}^{\Theta^{ \pm e l l} \mathrm{NF}}\right\}_{n, m \in \mathbb{Z}}$ be a collection of distinct $\Theta^{ \pm \mathrm{ell}} \mathrm{NF}$ Hodge theaters [relative to the given initial $\Theta$-data] indexed by pairs of integers. Then we shall refer to either of the diagrams

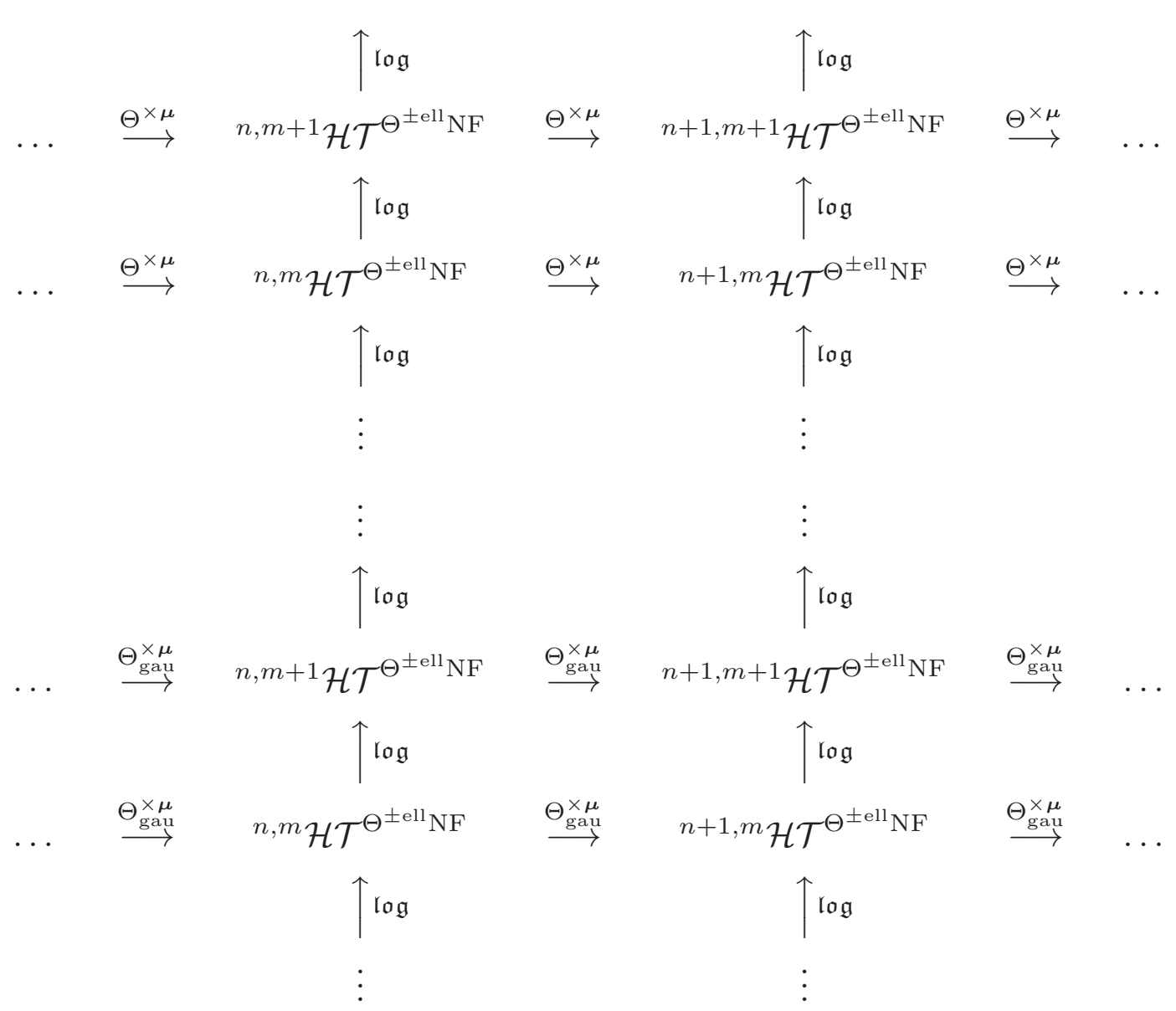

- where the vertical arrows are the full log-links, and the horizontal arrows are the $\Theta^{\times \mu_{-}}$and $\Theta_{\text {gau }}^{\times \boldsymbol{\mu}}$-links of [IUTchII], Corollary 4.10, (iii) — as the log-theta-lattice. We shall refer to the log-theta-lattice that involves the $\Theta^{\times \boldsymbol{\mu}_{-}}$(respectively, $\Theta_{\mathrm{gau}^{-}}$) links as non-Gaussian (respectively, Gaussian). Thus, either of these diagrams may be represented symbolically by an oriented graph

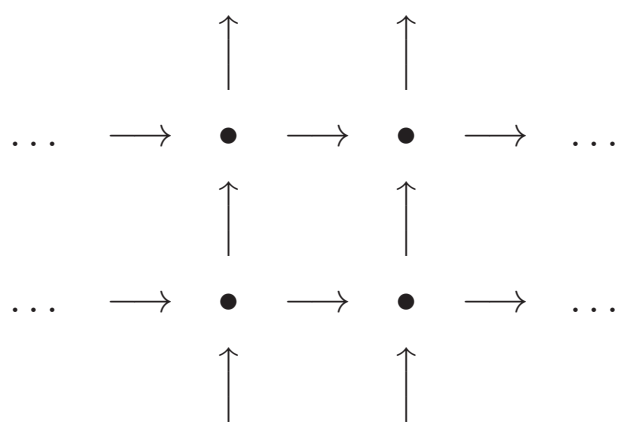


— where the "•'s" correspond to the "n,m $\mathcal{H} \mathcal{T}^{\Theta^{ \pm \text {ell }} \mathrm{NF}}$ ".

\section{Remark 1.4.1.}

(i) One fundamental property of the log-theta-lattices discussed in Definition 1.4 is the following:

the various squares that appear in each of the log-theta-lattices discussed in Definition 4.1 are far from being [1-]commutative!

Indeed, whereas the vertical arrows in each log-theta-lattice are constructed by applying the various logarithms at $\underline{v} \in \underline{\mathbb{V}}$ - i.e., which are defined by means of power series that depend, in an essential way, on the local ring structures at $\underline{v} \in \underline{\mathbb{V}}$

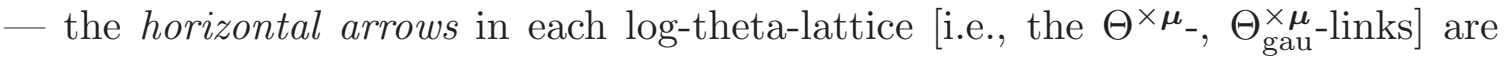
incompatible with these local ring structures at $\underline{v} \in \mathbb{V}$ in an essential way [cf. [IUTchII], Remark 1.11.2, (i), (ii)].

(ii) Whereas the horizontal arrows in each log-theta-lattice [i.e., the $\Theta^{\times \boldsymbol{\mu}_{-}}$, $\Theta_{\text {gau }}^{\times \boldsymbol{\mu}}$-links] allow one, roughly speaking, to identify the respective " $\mathcal{O} \times \boldsymbol{\mu}$ 's" at [for simplicity] $\underline{v} \in \underline{\mathbb{V}}^{\text {non }}$ on either side of the horizontal arrow [cf. [IUTchII], Corollary 4.10, (iv)], in order to avail oneself of the theory of log-shells - which will play an essential role in the multiradial representation of the Gaussian monoids to be

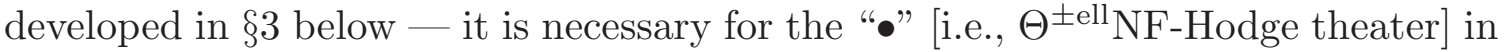
which one operates to appear as the codomain of a log-link, i.e., of a vertical arrow of the log-theta-lattice [cf. the discussion of [AbsTopIII], Remark 5.10.2, (iii)]. That is to say, from the point of view of the goal of constructing the multiradial representation of the Gaussian monoids that is to be developed in $\S 3$ below,

each execution of a horizontal arrow of the log-theta-lattice necessarily obligates a subsequent execution of a vertical arrow of the log-theta-lattice.

On the other hand, in light of the noncommutativity observed in (i), this "intertwining" of the horizontal and vertical arrows of the log-theta-lattice means that the desired multiradiality - i.e., simultaneous compatibility with the arithmetic holomorphic structures on both sides of a horizontal arrow of the logtheta-lattice - can only be realized if one works with objects that are invariant with respect to the vertical arrows [i.e., with respect to the action of $\mathbb{Z}$ discussed in Proposition 1.3, (iv)], that is to say, with "vertical cores", of the log-theta-lattice.

(iii) From the point of view of the analogy between the theory of the present series of papers and p-adic Teichmüller theory [cf. [AbsTopIII], §I5], the vertical arrows of the log-theta-lattice correspond to the Frobenius morphism in positive characteristic, whereas the horizontal arrows of the log-theta-lattice correspond to the "transition from $p^{n} \mathbb{Z} / p^{n+1} \mathbb{Z}$ to $p^{n-1} \mathbb{Z} / p^{n} \mathbb{Z}$ ", i.e., the mixed characteristic extension structure of a ring of Witt vectors [cf. [IUTchI], Remark 3.9.3, (i)]. These correspondences are summarized in Fig. 1.3 below. In particular, the "intertwining of horizontal and vertical arrows of the log-theta-lattice" discussed in (ii) above may be thought of as the analogue, in the context of the theory of the present series of papers, of the well-known "intertwining between the mixed characteristic 
extension structure of a ring of Witt vectors and the Frobenius morphism in positive characteristic" that appears in the classical $p$-adic theory.

\begin{tabular}{|c|c|}
\hline $\begin{array}{c}\text { horizontal arrows of the } \\
\text { log-theta-lattice }\end{array}$ & $\begin{array}{c}\text { mixed characteristic extension structure } \\
\text { of a ring of Witt vectors }\end{array}$ \\
\hline $\begin{array}{c}\text { vertical arrows of the } \\
\text { log-theta-lattice }\end{array}$ & $\begin{array}{c}\text { the Frobenius morphism } \\
\text { in positive characteristic }\end{array}$ \\
\hline
\end{tabular}

Fig. 1.3: Analogy between the log-theta-lattice and $p$-adic Teichmüller theory

\section{Remark 1.4.2.}

(i) The horizontal and vertical arrows of the log-theta-lattices discussed in Definition 4.1 share the common property of being incompatible with the local ring structures, hence, in particular, with the conventional scheme-theoretic basepoints on either side of the arrow in question [cf. the discussion of [IUTchII], Remark 3.6.3, (i)]. On the other hand, whereas the linking data of the vertical arrows [i.e., the loglink] is rigid and corresponds to a single fixed, rigid arithmetic holomorphic structure in which addition and multiplication are subject to "rotations" [cf. the discussion of [AbsTopIII], §I3], the linking data of the horizontal arrows [i.e., the $\Theta^{\times \mu_{-},} \Theta_{\text {gau }}^{\times \mu}$-links] - i.e., more concretely, the " $\mathcal{O}^{\times \mu}$ 's" at [for simplicity] $\underline{v} \in \underline{\mathbb{V}}^{\text {non }}$ — is subject to a $\widehat{\mathbb{Z}}^{\times}$-indeterminacy, which has the effect of obliterating the arithmetic holomorphic structure associated to a vertical line of the log-theta-lattice [cf. the discussion of [IUTchII], Remark 1.11.2, (i), (ii)].

(ii) If, in the spirit of the discussion of [IUTchII], Remark 1.11.2, (ii), one attempts to "force" the horizontal arrows of the log-theta-lattice to be compatible with the arithmetic holomorphic structures on either side of the arrow by declaring — in the style of the log-link! — that these horizontal arrows induce an isomorphism of the respective " $\Pi_{\underline{v}}$ 's" at [for simplicity] $\underline{v} \in \underline{\mathbb{V}}^{\text {non }}$, then one must contend with a situation in which the "common arithmetic holomorphic structure rigidified by the isomorphic copies of $\Pi_{\underline{v}}$ " is obliterated each time one takes into account the action of a nontrivial element of $\widehat{\mathbb{Z}}^{\times}$[i.e., that arises from the $\widehat{\mathbb{Z}}^{\times}$indeterminacy involved] on the corresponding " $\mathcal{O}^{\times \mu}$ ". In particular, in order to keep track of the arithmetic holomorphic structure currently under consideration, one must, in effect, consider paths that record the sequence of " $\Pi_{\underline{v}}$-rigidifying" and " $\widehat{\mathbb{Z}}^{\times}$-indeterminacy" operations that one invokes. On the other hand, the horizontal lines of the log-theta-lattices given in Definition 4.1 amount, in effect, to universal covering spaces of the loops - i.e., "unraveling paths of the loops" [cf. the discussion of Remark 1.2.2, (vi)] — that occur as one invokes various series of " $\Pi_{\underline{v}}$-rigidifying" and " $\widehat{\mathbb{Z}} \times$-indeterminacy" operations. Thus, in summary, any attempt as described above to "force" the horizontal arrows of the log-theta-lattice to be compatible with the arithmetic holomorphic structures on either side of the arrow does not result in any substantive simplification of the theory of the present 
series of papers. We refer the reader to [IUTchIV], Remark 3.6.3, for a discussion of a related topic.

We are now ready to state the main result of the present $\S 1$.

Theorem 1.5. (Bi-cores of the Log-theta-lattice) Fix a collection of initial $\Theta$-data

$$
\left(\bar{F} / F, X_{F}, l, \underline{C}_{K}, \underline{\mathbb{V}}, \underline{\epsilon}\right)
$$

as in [IUTchI], Definition 3.1. Then any Gaussian log-theta-lattice corresponding to this collection of initial $\Theta$-data [cf. Definition 1.4] satisfies the following properties:

(i) (Vertical Coricity) The vertical arrows of the Gaussian log-theta-lattice induce full poly-isomorphisms between the respective associated $\mathcal{D}$ - $\Theta^{ \pm \text {ell }} N F$-Hodge theaters

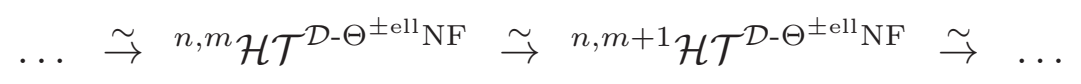

[cf. Proposition 1.3, (ii)]. Here, $n \in \mathbb{Z}$ is held fixed, while $m \in \mathbb{Z}$ is allowed to vary.

(ii) (Horizontal Coricity) The horizontal arrows of the Gaussian log-thetalattice induce full poly-isomorphisms between the respective associated $\mathcal{F}^{-\times \mu_{-}}$ prime-strips

$$
\ldots \quad \stackrel{\sim}{\rightarrow} n, m \mathfrak{F}_{\triangle}^{\vdash \times \mu} \stackrel{\sim}{\rightarrow} n+1, m \mathfrak{F}_{\triangle}^{\vdash \times \mu} \stackrel{\sim}{\rightarrow} \ldots
$$

[cf. [IUTchII], Corollary 4.10, (iv)]. Here, $m \in \mathbb{Z}$ is held fixed, while $n \in \mathbb{Z}$ is allowed to vary.

(iii) (Bi-coric $\mathcal{F}^{\vdash \times \mu}$-Prime-Strips) For $n, m \in \mathbb{Z}$, write ${ }^{n, m} \mathfrak{D}_{\triangle}^{\vdash}$ for the $\mathcal{D}^{\vdash}$ prime-strip associated to the $\mathcal{F}^{\vdash}$-prime-strip ${ }^{n, m} \mathfrak{F}_{\triangle}^{\vdash}$ labeled " $\triangle$ " of the $\Theta^{ \pm \text {ell }} N F$ Hodge theater ${ }^{n, m} \mathcal{H} \mathcal{T}^{\Theta^{ \pm \mathrm{ell}} \mathrm{NF}}$ [cf. [IUTchII], Corollary 4.10, (i)]; ${ }^{n, m} \mathfrak{D}_{\succ}$ for the $\mathcal{D}$ prime-strip labeled " $\succ$ " of the $\Theta^{ \pm \mathrm{ell}} \mathrm{NF}$-Hodge theater ${ }^{n, m} \mathcal{H} \mathcal{T}^{\Theta^{ \pm \mathrm{ell}} \mathrm{NF}}$ [cf. [IUTchI], Definition 6.11, (i)]. Let us identify [cf. [IUTchII], Corollary 4.10, (i)] the collections of data

$$
\Psi_{\mathrm{cns}}\left({ }^{n, m} \mathfrak{D}_{\succ}\right)_{0} \text { and } \Psi_{\mathrm{cns}}\left({ }^{n, m} \mathfrak{D}_{\succ}\right)_{\left\langle\mathbb{F}_{l}^{*}\right\rangle}
$$

via the isomorphism of the final display of [IUTchII], Corollary 4.5, (iii), and denote by

$$
\mathfrak{F}_{\triangle}^{\vdash}\left({ }^{n, m} \mathfrak{D}_{\succ}\right)
$$

the resulting $\mathcal{F}^{\vdash}$-prime-strip. [Thus, it follows immediately from the constructions involved - cf. the discussion of [IUTchII], Corollary 4.10, (i) - that there is a natural identification isomorphism $\mathfrak{F}_{\triangle}^{\vdash}\left(n, m \mathfrak{D}_{\succ}\right) \stackrel{\sim}{\rightarrow} \mathfrak{F}_{>}^{\vdash}\left(n, m \mathfrak{D}_{>}\right)$, where we write $\mathfrak{F}_{>}^{\vdash}\left(n, m \mathfrak{D}_{>}\right)$for the $\mathcal{F}^{\vdash}$-prime-strip determined by $\Psi_{\text {cns }}\left(n, m \mathfrak{D}_{>}\right)$.] Write

$$
\mathfrak{F}_{\triangle}^{\vdash \times}\left({ }^{n, m} \mathfrak{D}_{\succ}\right), \quad \mathfrak{F}_{\triangle}^{\vdash \times \boldsymbol{\mu}}\left({ }^{n, m} \mathfrak{D}_{\succ}\right)
$$


for the $\mathcal{F}^{\vdash \times}-, \mathcal{F}^{\vdash \times \boldsymbol{\mu}}$-prime-strips determined by $\mathfrak{F}_{\triangle}^{\vdash}\left({ }^{n, m} \mathfrak{D}_{\succ}\right)$ [cf. [IUTchII], Definition 4.9, (vi), (vii)]. Thus, by applying the isomorphisms " $\Psi_{\mathrm{cns}}\left({ }^{n, m} \mathfrak{D}\right)_{\underline{v}}^{\times} \stackrel{\sim}{\rightarrow}$ $\Psi_{\mathrm{cns}}^{\mathrm{ss}}\left(n, m \mathfrak{D}^{\vdash}\right)_{\underline{v}}^{\times} "$, for $\underline{v} \in \underline{\mathbb{V}}$, of [IUTchII], Corollary 4.5, (ii), [it follows immediately from the definitions that] there exists a functorial algorithm in the $\mathcal{D}^{\vdash}$-primestrip $^{n, m} \mathfrak{D}_{\triangle}^{\vdash}$ for constructing an $\mathcal{F}^{\vdash \times}$-prime-strip $\mathfrak{F}_{\triangle}^{\vdash \times}\left({ }^{n, m} \mathfrak{D}_{\triangle}^{\vdash}\right)$, together with a natural isomorphism

$$
\mathfrak{F}_{\triangle}^{\vdash \times}\left({ }^{n, m} \mathfrak{D}_{\succ}\right) \stackrel{\sim}{\rightarrow} \mathfrak{F}_{\triangle}^{\vdash \times}\left(n, m \mathfrak{D}_{\triangle}^{\vdash}\right)
$$

- i.e., in more intuitive terms, " $\mathfrak{F}_{\triangle}^{\vdash \times}\left(n, m \mathfrak{D}_{\succ}\right)$ ", hence also the associated $\mathcal{F}^{\vdash \times \boldsymbol{\mu}_{-}}$ prime-strip " $\mathfrak{F}_{\triangle} \times \boldsymbol{\mu}\left({ }^{n, m} \mathfrak{D}_{\succ}\right)$ ", may be naturally regarded as objects constructed from $n, m \mathfrak{D}_{\triangle}^{\vdash}$. Then the poly-isomorphisms of (i) [cf. Remark 1.3.2], (ii) induce, respectively, poly-isomorphisms of $\mathcal{F}^{\vdash \times \boldsymbol{\mu}}$-prime-strips

$$
\begin{aligned}
& \ldots \stackrel{\sim}{\rightarrow} \mathfrak{F}_{\triangle}^{\vdash \times \boldsymbol{\mu}}\left({ }^{n, m} \mathfrak{D}_{\succ}\right) \stackrel{\sim}{\rightarrow} \mathfrak{F}_{\triangle}^{\vdash \times \boldsymbol{\mu}}\left({ }^{n, m+1} \mathfrak{D}_{\succ}\right) \stackrel{\sim}{\rightarrow} \ldots \\
& \ldots \stackrel{\sim}{\rightarrow} \mathfrak{F}_{\triangle}^{\vdash \times \boldsymbol{\mu}}\left({ }^{n, m} \mathfrak{D}_{\triangle}^{\vdash}\right) \stackrel{\sim}{\rightarrow} \mathfrak{F}_{\triangle}^{\vdash \times \boldsymbol{\mu}}\left({ }^{n+1, m} \mathfrak{D}_{\triangle}^{\vdash}\right) \stackrel{\sim}{\rightarrow} \ldots
\end{aligned}
$$

- where we note that the poly-isomorphisms of $\mathcal{F}^{\vdash \times \boldsymbol{\mu}}$-prime-strips of the first line of the display are not full. In particular, by composing these isomorphisms, one obtains full poly-isomorphisms of $\mathcal{F}^{\vdash \times \boldsymbol{\mu}}$-prime-strips

$$
\mathfrak{F}_{\triangle}^{\vdash} \times \boldsymbol{\mu}\left(n, m \mathfrak{D}_{\triangle}^{\vdash}\right) \stackrel{\sim}{\rightarrow} \mathfrak{F}_{\triangle}^{\vdash} \times \boldsymbol{\mu}\left(n^{\prime}, m^{\prime} \mathfrak{D}_{\triangle}^{\vdash}\right)
$$

for arbitrary $n^{\prime}, m^{\prime} \in \mathbb{Z}$. That is to say, in more intuitive terms, the $\mathcal{F}^{\vdash \times \boldsymbol{\mu}}$-primestrip " $n, m \mathfrak{F}_{\triangle}^{\vdash} \times \boldsymbol{\mu}\left(n, m \mathfrak{D}_{\triangle}^{\vdash}\right)$ ", regarded up to isomorphism, is an invariant - which we shall refer to as "bi-coric" - of both the horizontal and the vertical arrows of the log-theta-lattice. Finally, the Kummer isomorphisms " $\Psi_{\mathrm{cns}}\left({ }^{\ddagger} \mathfrak{F}\right) \stackrel{\sim}{\rightarrow} \Psi_{\mathrm{cns}}\left({ }^{\ddagger} \mathfrak{D}\right)$ " of [IUTchII], Corollary 4.6, (i), determine Kummer isomorphisms

$$
n, m \underset{\mathfrak{F}_{\triangle}}{\vdash} \times \stackrel{\sim}{\rightarrow} \mathfrak{F}_{\triangle}^{\vdash} \times \boldsymbol{\mu}\left(n, m \mathfrak{D}_{\triangle}^{\vdash}\right)
$$

which are compatible with the poly-isomorphisms of (ii), as well as with the $\times \boldsymbol{\mu}$ Kummer structures at the $\underline{v} \in \underline{\mathbb{V}}^{\text {non }}$ of the various $\mathcal{F}^{\vdash \times \boldsymbol{\mu}}$-prime-strips involved [cf. [IUTchII], Definition 4.9, (vi), (vii)]; a similar compatibility holds for $\underline{v} \in \underline{\mathbb{V}}^{\text {arc }}$ [cf. the discussion of the final portion of [IUTchII], Definition 4.9, (v)].

(iv) (Bi-coric Mono-analytic Log-shells) The full poly-isomorphisms that constitute the bi-coricity property discussed in (iii) induce poly-isomorphisms

$$
\begin{aligned}
&\left\{\mathcal{I}_{n, m} \mathfrak{D}_{\triangle}^{\vdash}\right.\left.\subseteq \underline{\log }\left(n^{n, m} \mathfrak{D}_{\triangle}^{\vdash}\right)\right\} \stackrel{\sim}{\rightarrow}\left\{\mathcal{I}_{n^{\prime}, m^{\prime} \mathfrak{D}_{\triangle}^{\vdash}} \subseteq \underline{\log }\left(n^{\prime}, m^{\prime} \mathfrak{D}_{\triangle}^{\vdash}\right)\right\} \\
&\left\{\mathcal{I}_{\mathfrak{F}_{\triangle}^{\vdash \times \mu}\left(n, m \mathfrak{D}_{\triangle}^{\vdash}\right)} \subseteq \underline{\mathfrak{l o g}}\left(\mathfrak{F}_{\triangle}^{\vdash \times \mu}\left(n, m \mathfrak{D}_{\triangle}^{\vdash}\right)\right)\right\} \stackrel{\sim}{\rightarrow}\left\{\mathcal{I}_{\mathfrak{F}_{\triangle}^{\vdash \times \mu}\left(n^{\prime}, m^{\prime} \mathfrak{D}_{\triangle}^{\vdash}\right)} \subseteq \underline{\mathfrak{l o g}}\left(\mathfrak{F}_{\triangle}^{\vdash \times \mu}\left(n^{\prime}, m^{\prime} \mathfrak{D}_{\triangle}^{\vdash}\right)\right)\right\}
\end{aligned}
$$

for arbitrary $n, m, n^{\prime}, m^{\prime} \in \mathbb{Z}$ that are compatible with the natural poly-isomorphisms

$$
\left\{\mathcal{I}_{n, m \mathfrak{D}_{\triangle}^{\vdash}} \subseteq \underline{\mathfrak{l o g}}\left({ }^{n, m} \mathfrak{D}_{\triangle}^{\vdash}\right)\right\} \stackrel{\sim}{\rightarrow}\left\{\mathcal{I}_{\mathfrak{F}_{\triangle}^{\vdash \times \mu}\left(n, m \mathfrak{D}_{\triangle}^{\vdash}\right)} \subseteq \underline{\mathfrak{l o g}}\left(\mathfrak{F}_{\triangle}^{\vdash \times \boldsymbol{\mu}}\left({ }^{n, m} \mathfrak{D}_{\triangle}^{\vdash}\right)\right)\right\}
$$


of Proposition 1.2, (viii). On the other hand, by applying the constructions of Definition 1.1, (i), (ii), to the collections of data " $\Psi_{\mathrm{cns}}\left({ }^{\dagger} \mathfrak{F}_{\succ}\right)_{0}$ " and " $\Psi_{\mathrm{cns}}\left({ }^{\dagger} \mathfrak{F}_{\succ}\right)_{\left\langle\mathbb{F}_{l}^{*}\right\rangle}$ " used in [IUTchII], Corollary 4.10, (i), to construct ${ }^{n, m} \mathfrak{F}_{\triangle}^{\vdash}$ [cf. Remark 1.3.2], one obtains a ["holomorphic"] log-shell, together with an enveloping "log $(-)$ " [cf. the pair " $\mathcal{I}_{\dagger} \mathfrak{F} \subseteq \underline{\log }\left({ }^{\dagger} \mathfrak{F}\right)$ " of Definition 1.1, (iii)], which we denote by

$$
\mathcal{I}_{n, m} \mathfrak{F}_{\triangle} \subseteq \underline{\log }\left({ }^{n, m} \mathfrak{F}_{\triangle}\right)
$$

[by means of a slight abuse of notation, since no $\mathcal{F}$-prime-strip "n,m $\mathfrak{F} \triangle$ " has been defined!]. Then one has natural poly-isomorphisms

$$
\begin{aligned}
\left\{\mathcal{I}_{n, m} \mathfrak{D}_{\triangle}^{\vdash} \subseteq \underline{\log }\left({ }^{n, m} \mathfrak{D}_{\triangle}^{\vdash}\right)\right\} & \stackrel{\sim}{\rightarrow}\left\{\mathcal{I}_{n, m} \mathfrak{F}_{\triangle}^{\vdash \times \mu} \subseteq \underline{\mathfrak{l o g}}\left({ }^{n, m} \mathfrak{F}_{\triangle}^{\vdash \times \mu}\right)\right\} \\
& \stackrel{\sim}{\rightarrow}\left\{\mathcal{I}_{n, m} \mathfrak{F}_{\triangle} \subseteq \underline{\mathfrak{l o g}}\left({ }^{n, m} \mathfrak{F}_{\triangle}\right)\right\}
\end{aligned}
$$

[cf. the poly-isomorphisms obtained in Proposition 1.2, (viii)]; here, the first " $\underset{\rightarrow}{\rightarrow}$ may be regarded as being induced by the Kummer isomorphisms of (iii) and is compatible with the poly-isomorphisms induced by the poly-isomorphisms of (ii).

(v) (Bi-coric Mono-analytic Realified Global Frobenioids) Let $n, m, n^{\prime}, m^{\prime} \in$ $\mathbb{Z}$. Then the poly-isomorphisms of $\mathcal{D}^{\vdash}$-prime-strips ${ }^{n, m} \mathfrak{D}_{\triangle}^{\vdash} \stackrel{\sim}{\rightarrow} n^{\prime}, m^{\prime} \mathfrak{D}_{\triangle}^{\vdash}$ induced by the full poly-isomorphisms of (i), (ii) induce [cf. [IUTchII], Corollaries 4.5, (ii); $4.10,(v)]$ an isomorphism of collections of data

$$
\begin{aligned}
\left(\mathcal{D}^{\Vdash}(\right. & \left.\left(n, m \mathfrak{D}_{\triangle}^{\vdash}\right), \operatorname{Prime}\left(\mathcal{D}^{\Vdash}\left(n, m \mathfrak{D}_{\triangle}^{\vdash}\right)\right) \stackrel{\sim}{\rightarrow} \underline{\mathbb{V}},\left\{{ }^{n, m} \rho_{\mathcal{D}^{\Vdash}, \underline{v}}\right\}_{\underline{v}} \in \underline{\mathbb{V}}\right) \\
& \stackrel{\sim}{\rightarrow} \quad\left(\mathcal{D}^{\Vdash}\left(n^{\prime}, m^{\prime} \mathfrak{D}_{\triangle}^{\vdash}\right), \operatorname{Prime}\left(\mathcal{D}^{\Vdash}\left(n^{\prime}, m^{\prime} \mathfrak{D}_{\triangle}^{\vdash}\right)\right) \stackrel{\sim}{\rightarrow} \underline{\mathbb{V}},\left\{\left\{^{n^{\prime}, m^{\prime}} \rho_{\mathcal{D}^{\Vdash}, \underline{v}}\right\}_{\underline{v} \in \underline{\mathbb{V}}}\right)\right.
\end{aligned}
$$

- i.e., consisting of a Frobenioid, a bijection, and a collection of isomorphisms of topological monoids indexed by $\underline{\mathbb{V}}$. Moreover, this isomorphism of collections of data is compatible, relative to the full poly-isomorphisms of (ii), with the $\mathbb{R}_{>0}$-orbits of the isomorphisms of collections of data

$$
\begin{aligned}
\left({ }^{n, m} \mathcal{C}_{\triangle}^{\Vdash},\right. & \left.\operatorname{Prime}\left({ }^{n, m} \mathcal{C}_{\triangle}^{\Vdash}\right) \stackrel{\sim}{\rightarrow} \underline{\mathbb{V}},\left\{{ }^{n, m} \rho_{\triangle, \underline{v}}\right\}_{\underline{v} \in \underline{\mathbb{V}}}\right) \\
& \stackrel{\sim}{\rightarrow}\left(\mathcal{D}^{\Vdash}\left({ }^{n, m} \mathfrak{D}_{\triangle}^{\vdash}\right), \operatorname{Prime}\left(\mathcal{D}^{\Vdash}\left({ }^{n, m} \mathfrak{D}_{\triangle}^{\vdash}\right)\right) \stackrel{\sim}{\rightarrow} \underline{\mathbb{V}},\left\{{ }^{n, m} \rho_{\mathcal{D}^{\Vdash}, \underline{v}}\right\}_{\underline{v} \in \underline{\mathbb{V}}}\right)
\end{aligned}
$$

obtained by applying the functorial algorithm discussed in the final portion of [IUTchII], Corollary 4.6, (ii) [cf. also the final portion of [IUTchII], Corollary 4.10, (v)].

Proof. The various assertions of Theorem 1.5 follow immediately from the definitions and the references quoted in the statements of these assertions.

\section{Remark 1.5.1.}

(i) Note that the theory of conjugate synchronization developed in [IUTchII] [cf., especially, [IUTchII], Corollaries 4.5, (iii); 4.6, (iii)] plays an essential role in establishing the bi-coricity properties discussed in Theorem 1.5, (iii), (iv) - i.e., at a more technical level, in constructing the objects equipped with a subscript " $\triangle$ " 
that appear in Theorem 1.5, (iii); [IUTchII], Corollary 4.10, (i). That is to say, the conjugate synchronization determined by the various symmetrizing isomorphisms of [IUTchII], Corollaries 4.5, (iii); 4.6, (iii), may be thought of as a sort of descent mechanism that allows one to descend data that, a priori, is label-dependent [i.e., depends on the labels " $t \in \operatorname{LabCusp}^{ \pm}(-)$"] to data that is label-independent. Here, it is important to recall that these labels depend, in an essential way, on the "arithmetic holomorphic structures" involved - i.e., at a more technical level, on the geometric fundamental groups involved - hence only make sense within a vertical line of the log-theta-lattice. That is to say, the significance of this transition from label-dependence to label-independence lies in the fact that this transition is precisely what allows one to construct objects that make sense in horizontally adjacent "•'s" of the log-theta-lattice, i.e., to construct horizontally coric objects [cf. Theorem 1.5, (ii); the second line of the fifth display of Theorem 1.5, (iii)]. On the other hand, in order to construct the horizontal arrows of the log-theta-lattice, it is necessary to work with Frobenius-like structures [cf. the discussion of [IUTchII], Remark 3.6.2, (ii)]. In particular, in order to construct vertically coric objects [cf. the first line of the fifth display of Theorem 1.5, (iii)], it is necessary to pass to étale-like structures [cf. the discussion of Remark 1.2.4, (i)] by means of Kummer isomorphisms [cf. the final display of Theorem 1.5, (iii)]. Thus, in summary,

the bi-coricity properties discussed in Theorem 1.5, (iii), (iv) - i.e., roughly speaking, the bi-coricity of the various "Oํ $\times$ " at $\underline{v} \in \underline{\mathbb{V}}^{\text {non }}$ may be thought of as a consequence of the intricate interplay of various aspects of the theory of Kummer-compatible conjugate synchronization established in [IUTchII], Corollaries 4.5, (iii); 4.6, (iii).

(ii) In light of the central role played by the theory of conjugate synchronization in the constructions that underlie Theorem 1.5 [cf. the discussion of (i)], it is of interest to examine in more detail to what extent the highly technically nontrivial theory of conjugate synchronization may be replaced by a simpler apparatus. One naive approach to this problem is the following. Let $G$ be a topological group [such as one of the absolute Galois groups $G_{\underline{v}}$ associated to $\left.\underline{v} \in \underline{\mathbb{V}}^{\text {non }}\right]$. Then one way to attempt to avoid the application of the theory of conjugate synchronization which amounts, in essence, to the construction of a diagonal embedding

$$
G \hookrightarrow G \times \ldots \times G
$$

[cf. the notation " $\left\langle\left|\mathbb{F}_{l}\right|\right\rangle "$, " $\left\langle\mathbb{F}_{l}^{*}\right\rangle$ " that appears in [IUTchII], Corollaries 3.5, 3.6, $4.5,4.6]$ in a product of copies of $G$ that, a priori, may only be identified with one another up to conjugacy [i.e., up to composition with an inner automorphism] - is to try to work, instead, with the $(G \times \ldots \times G)$-conjugacy class of such a diagonal. Here, to simplify the notation, let us assume that the above products of copies of $G$ are, in fact, products of two copies of $G$. Then to identify the diagonal embedding $G \hookrightarrow G \times G$ with its $(G \times G)$-conjugates implies that one must consider identifications

$$
(g, g) \sim\left(g, h g h^{-1}\right)=(g,[h, g] \cdot g)
$$

[where $g, h \in G]$ - i.e., one must identify $(g, g)$ with the product of $(g, g)$ with $(1,[h, g])$. On the other hand, the original purpose of working with distinct copies 
of $G$ lies in considering distinct Galois-theoretic Kummer classes - corresponding to distinct theta values [cf. [IUTchII], Corollaries 3.5, 3.6] - at distinct components. That is to say, to identify elements of $(G, G)$ that differ by a factor of $(1,[h, g])$ is incompatible, in an essential way, with the convention that such a factor $(1,[h, g])$ should correspond to distinct elements [i.e., "1" and " $[h, g]$ "] at distinct components [cf. the discussion of Remark 1.5.3, (ii), below]. Here, we note that this incompatibility may be thought of as an essential consequence of the highly nonabelian nature of $G$, e.g., when $G$ is taken to a copy of $G_{\underline{v}}$, for $\underline{v} \in \underline{\mathbb{V}}^{\text {non }}$. Thus, in summary, this naive approach to replacing the theory of conjugate synchronization by a simpler apparatus is inadequate from the point of view of the theory of the present series of papers.

(iii) At a purely combinatorial level, the notion of conjugate synchronization is reminiscent of the label synchronization discussed in [IUTchI], Remark 4.9.2, (i), (ii). Indeed, both conjugate and label synchronization may be thought of as a sort of combinatorial representation of the arithmetic holomorphic structure associated to a single vertical line of the log-theta-lattice [cf. the discussion of [IUTchI], Remark 4.9.2, (iv)].

\section{Remark 1.5.2.}

(i) Recall that unlike the case with the action of the $\mathbb{F}_{l}^{\rtimes \pm}$-symmetry on the various labeled copies of the absolute Galois group $G_{\underline{v}}$, for $\underline{v} \in \underline{\mathbb{V}}^{\text {non }}[$ cf. [IUTchII], Corollaries 4.5, (iii); 4.6, (iii)], it is not possible to establish an analogous theory of conjugate synchronization in the case of the $\mathbb{F}_{l}^{*}$-symmetry for labeled copies of $\bar{F}$ [cf. [IUTchII], Remark 4.7.2]. This is to say, the closest analogue of the conjugate synchronization obtained in the local case relative to the $\mathbb{F}_{l}^{\rtimes \pm}$-symmetry is the action of the $\mathbb{F}_{l}^{*}$-symmetry on labeled copies of the subfield $F_{\text {mod }} \subseteq \bar{F}$ discussed in [IUTchII], Corollaries 4.7, (ii); 4.8, (ii). One consequence of this incompatibility of the $\mathbb{F}_{l}^{*}$-symmetry with the full algebraic closure $\bar{F}$ of $F_{\bmod }$ [hence, in particular, with various roots of elements of $\left.F_{\text {mod }}\right]$ is that the $\mathbb{F}_{l}^{*}$-symmetry on labeled copies of the subfield $F_{\text {mod }} \subseteq \bar{F}$ discussed in [IUTchII], Corollaries 4.7, (ii); 4.8, (ii), fails to be compatible with Kummer theory. This is one quite essential reason why it is not possible to establish bi-coricity properties for, say, " $F_{\bmod }^{\times}$" that are analogous to the bi-coricity properties established in Theorem 1.5 , (iii), for " $\mathcal{O}^{\times \mu}$ " [cf. the discussion of Remark 1.5.1, (i)]. From this point of view,

the bi-coric mono-analytic realified global Frobenioids of Theorem $1.5,(\mathrm{v})$ - i.e., in essence, the notion of "log-volume" [cf. the point of view of Remark 1.2.2, (v)] — may be thought of as a sort of "closest possible approximation" to such a "bi-coric $F_{\bmod }^{\times}$" [i.e., which does not exist].

Alternatively, from the point of view of the theory to be developed in $\S 3$ below,

we shall apply the bi-coric " $\mathrm{O}^{\times \boldsymbol{\mu}}$ ' $\mathbf{s}$ " of Theorem 1.5 , (iii) - i.e., in the form of the bi-coric mono-analytic log-shells of Theorem 1.5, (iv) - to construct "multiradial containers" for the labeled copies of 
$F_{\text {mod }}$ discussed above by applying the localization functors discussed in [IUTchII], Corollaries 4.7, (iii); 4.8, (iii).

That is to say, such "multiradial containers" will play the role of a transportation mechanism for " $F_{\text {mod }}^{\times}$" - up to certain indeterminacies! — between distinct arithmetic holomorphic structures [i.e., distinct vertical lines of the log-theta-lattice].

(ii) Note that although the $\mathbb{F}_{l}^{*}$-symmetry fails to be compatible with labeled copies of " $\bar{F} "$ [cf. the discussion of (i)], if one forgets the $\mathbb{F}_{l}^{*}$-symmetry and the corresponding labels, then

the functorial algorithms of [IUTchII], Corollary 4.7, (i), applied to the vertical coricity of Theorem 1.5, (i), allow one to construct vertically coric versions of " $\bar{F}$ " which are, moreover, compatible with Kummer theory [cf. [IUTchII], Corollary 4.8, (i)].

This point of view will be applied in $\S 3$ below, in conjunction with the point of view of "multiradial containers" discussed in (i) above. Here, we recall [cf. the discussion of [IUTchII], Remark 3.6.2, (ii)] that, in general, the phenomenon of "Kummer-incompatibility" [i.e., incompatibility with Kummer theory — cf. the discussion of "Kummer-blindness" in [IUTchI], Remark 5.1.3] occurs precisely when one wishes to perform constructions - such as, for instance, the construction of the $\Theta_{-}, \Theta^{\times \boldsymbol{\mu}_{-}}$, or $\Theta_{\text {gau }}^{\times \boldsymbol{\mu}_{-}}$-links - that are "not bound to conventional scheme theory". That is to say, in the case of the labeled copies of " $F_{\text {mod" }}$ discussed in (i), the Kummer-incompatibility that occurs may be thought of as a reflection of the dismantling of the global prime-tree structure of a number field [cf. the discussion of [IUTchII], Remark 4.11.2, (iv)] that underlies the construction of the $\Theta^{ \pm \text {ell }}$ NF-Hodge theater performed in [IUTchI], [IUTchII], hence, in particular, of the conjugate synchronization established for the $\mathbb{F}_{l}^{\rtimes \pm}$-symmetry.

(iii) Despite the failure of labeled copies of " $F_{\bmod }^{\times}$" to admit a natural bi-coric structure - a state of affairs that forces one to resort to the use of "multiradial containers" in order to transport such labeled copies of " $F_{\bmod }^{\times}$" to alien arithmetic holomorphic structures [cf. the discussion of (i) above] - the global Frobenioids associated to copies of " $F_{\text {mod }}^{\times}$" nevertheless possess important properties that are not satisfied, for instance, by the bi-coric realified global Frobenioids discussed in Theorem 1.5, (v) [cf. also [IUTchI], Definition 5.2, (iv); [IUTchII], Corollary 4.5, (ii); [IUTchII], Corollary 4.6, (ii)]. Indeed, unlike the objects contained in the realified global Frobenioids that appear in Theorem 1.5, (v), the objects contained in the global Frobenioids associated to copies of " $F_{\bmod }^{\times}$" correspond to genuine "conventional arithmetic line bundles". In particular, by applying the ring structure of the copies of " $F_{\text {mod" }}$ " under consideration, one can push forward such arithmetic line bundles so as to obtain arithmetic vector bundles over [the ring of rational integers] $\mathbb{Z}$ and then form tensor products of such arithmetic vector bundles. Such operations will play a key role in the theory of $\S 3$ below, as well as in the theory to be developed in [IUTchIV]. 


\section{Remark 1.5.3.}

(i) In [QuCnf] [cf. also [AbsTopIII], Proposition 2.6; [AbsTopIII], Corollary 2.7], a theory was developed concerning deformations of holomorphic structures on Riemann surfaces in which holomorphic structures are represented by means of squares or rectangles on the surface, while quasiconformal Teichmüller deformations of holomorphic structures are represented by parallelograms on the surface. That is to say, relative to appropriate choices of local coordinates, quasiconformal Teichmüller deformations may be thought of as affine linear deformations in which one of the two underlying real dimensions of the Riemann surface is dilated by some factor $\in \mathbb{R}_{>0}$, while the other underlying real dimensions is left undeformed. From this point of view, the theory of conjugate synchronization — which may be regarded as a sort of ridigity that represents the arithmetic holomorphic structure associated to a vertical line of the log-theta-lattice [cf. the discussion given in [IUTchII], Remarks 4.7.3, 4.7.4, of the uniradiality of the $\mathbb{F}_{l}^{\rtimes \pm}$-symmetry that underlies the phenomenon of conjugate synchronization] — may be thought of as a sort of nonarchimedean arithmetic analogue of the representation of holomorphic structures by means of squares/rectangles referred to above. That is to say, the right angles which are characteristic of squares/rectangles may be thought of as a sort of synchronization between the metrics of the two underlying real dimensions of a Riemann surface [i.e., metrics which, a priori, may differ by some dilating factor] — cf. Fig. 1.4 below. Here, we mention in passing that this point of view is reminiscent of the discussion of [IUTchII], Remark 3.6.5, (ii), in which the point of view is taken that the phenomenon of conjugate synchronization may be thought of as a reflection of the coherence of the arithmetic holomorphic structures involved.

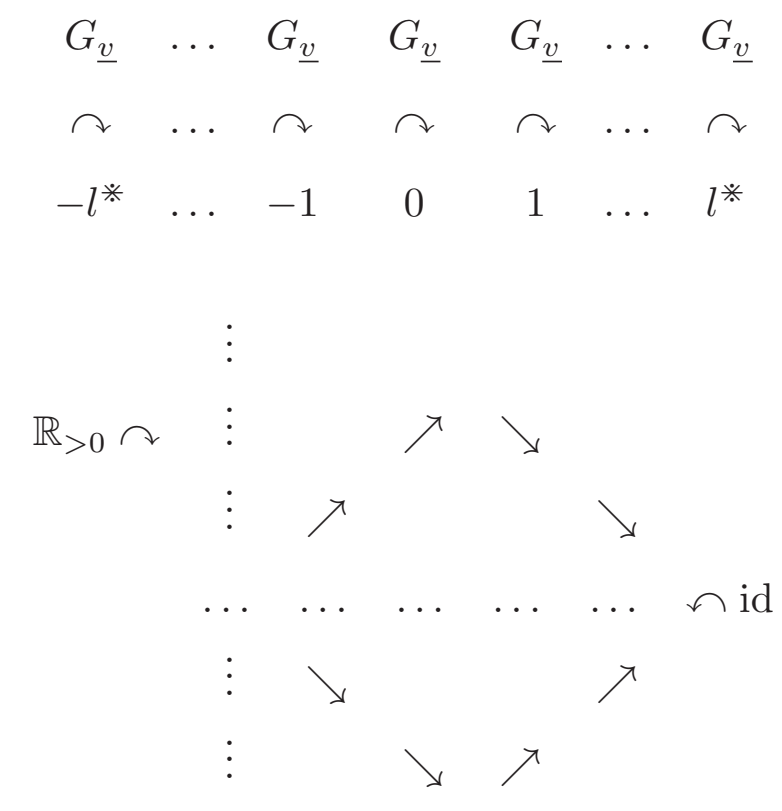

Fig. 1.4: Analogy between conjugate synchronization and the representation of complex holomorphic structures via squares/rectangles

(ii) Relative to the point of view discussed in (i), the approach described in Remark 1.5.1, (ii), to "avoiding conjugate synchronization by identifying the various 
conjugates of the diagonal embedding" corresponds — in light of the highly nonabelian nature of the groups involved! [cf. the discussion of Remark 1.5.1, (ii)] — to thinking of a holomorphic structure on a Riemann surface as an "equivalence class of holomorphic structures in the usual sense relative to the equivalence relation of differing by a Teichmüller deformation"! That is to say, such an [unconventional!] approach to the definition of a holomorphic structure allows one to circumvent the issue of rigidifying the relationship between the metrics of the two underlying real dimensions of the Riemann surface - but only at the cost of rendering unfeasible any meaningful theory of "deformations of a holomorphic structure"!

(iii) The analogy discussed in (i) between conjugate synchronization [which arises from the $\mathbb{F}_{l}^{\rtimes \pm}$-symmetry!] and the representation of a complex holomorphic structure by means of squares/rectangles may also be applied to the "synchronization" given in [IUTchII], Corollary 4.7, (ii); [IUTchII], Corollary 4.8, (ii), between the various labeled non-realified and realified global Frobenioids by means of the $\mathbb{F}_{l}^{*}$-symmetry. Indeed, this analogy is all the more apparent in the case of the realified global Frobenioids — which admit a natural $\mathbb{R}_{>0}$-action. Here, we observe in passing that, just as the theory of conjugate synchronization plays an essential role in the construction of the local portions of the $\Theta^{\times \boldsymbol{\mu}_{-}}, \Theta_{\mathrm{gau}}^{\times \boldsymbol{\mu}}$-links given in [IUTchII], Corollary 4.10, (i), (ii), (iii),

the synchronization of realified global Frobenioids by means of the $\mathbb{F}_{l}^{*}$-symmetry may be related - via the isomorphisms of Frobenioids of the second displays of [IUTchII], Corollary 4.7, (iii); [IUTchII], Corollary 4.8, (iii) [cf. also the discussion of [IUTchII], Remark 4.8.1] — to the construction of the realified global Frobenioid portion of the $\Theta_{\mathrm{gau}}^{\times \boldsymbol{\mu}}$-link given in [IUTchII], Corollary 4.10, (ii).

On the other hand, the synchronization involving the non-realified global Frobenioids may be thought of a sort of further rigidification of the realified global Frobenioids. As discussed in Remark 1.5.2, (iii), this "further rigidification" will play an important role in the theory of $\S 3$ below.

\section{Remark 1.5.4.}

(i) As discussed in [IUTchII], Remark 3.8.3, (iii), one of the main themes of the present series of papers is the goal of giving an explicit description of what one arithmetic holomorphic structure - i.e., one vertical line of the log-theta-lattice looks like from the point of view of a distinct arithmetic holomorphic structure - i.e., another vertical line of the log-theta-lattice - that is only related to the original arithmetic holomorphic structure via some mono-analytic core, e.g., the various bi-coric structures discussed in Theorem 1.5, (iii), (iv), (v). Typically, the objects of interest that are constructed within the original arithmetic holomorphic structure are Frobenius-like structures [cf. the discussion of [IUTchII], Remark 3.6.2], which, as we recall from the discussion of Remark 1.5.2, (ii) [cf. also the discussion of [IUTchII], Remark 3.6.2, (ii)], are necessary in order to perform constructions - such as, for instance, the construction of the $\Theta_{-}, \Theta^{\times \boldsymbol{\mu}_{-}}$, or $\Theta_{\mathrm{gau}}^{\times \boldsymbol{\mu}_{-}}$-links - that are "not bound to conventional scheme theory". Indeed, the main example of such an object of interest consists precisely of the Gaussian monoids 
discussed in [IUTchII], $33, \S 4$. Thus, the operation of describing such an object of interest from the point of view of a distinct arithmetic holomorphic structure may be broken down into two steps:

(a) passing from Frobenius-like structures to étale-like structures via various Kummer isomorphisms;

(b) transporting the resulting étale-like structures from one arithmetic holomorphic structure to another by means of various multiradiality properties.

In particular, the computation of what the object of interest looks like from the point of view of a distinct arithmetic holomorphic structure may be broken down into the computation of the indeterminacies or "departures from rigidity" that arise - i.e., the computation of "what sort of damage is incurred to the object of interest" - during the execution of each of these two steps (a), (b). We shall refer to the indeterminacies that arise from (a) as Kummer-detachment indeterminacies and to the indeterminacies that arise from (b) as étale-transport indeterminacies.

(ii) Etale-transport indeterminacies typically amount to the indeterminacies that occur as a result of the execution of various "anabelian" or "group-theoretic" algorithms. One fundamental example of such indeterminacies is constituted by the indeterminacies that occur in the context of Theorem 1.5, (iii), (iv), as a result of the existence of automorphisms of the various [copies of] local absolute Galois groups $G_{\underline{v}}$, for $\underline{v} \in \underline{\mathbb{V}}^{\text {non }}$, which are not of scheme-theoretic origin [cf. the discussion of [AbsTōiII], §I3].

(iii) On other hand, one important example, from the point of view of the theory of the present series of papers, of a Kummer-detachment indeterminacy is constituted by the Frobenius-picture diagrams given in Propositions 1.2, (x); 1.3, (iv) - i.e., the issue of which path one is to take from a particular "•" to the coric "o". That is to say, despite the fact that these diagrams fail to be commutative, the "upper semi-commutativity" property satisfied by the coric holomorphic log-shells involved [cf. the discussion of Remark 1.2.2, (iii)] may be regarded as a sort of computation, in the form of an upper estimate, of the Kummer-detachment indeterminacy in question. Another important example, from the point of view of the theory of the present series of papers, of a Kummer-detachment indeterminacy is given by the $\widehat{\mathbb{Z}}^{\times}$-indeterminacies discussed in Remark 1.4 .2 [cf. also the Kummer isomorphisms of the final display of Theorem 1.5, (iii)]. 


\section{Section 2: Multiradial Theta Monoids}

In the present $\S 2$, we globalize the multiradial portion of the local theory of theta monoids developed in [IUTchII], $\S 1, \S 3$, at $\underline{v} \in \mathbb{V}^{\text {bad }}$ [cf., especially, [IUTchII], Corollary 1.12; [IUTchII], Proposition 3.4] so as to cover the theta monoids of [IUTchII], Corollaries 4.5, (iv), (v); 4.6, (iv), (v), and explain how the resulting theory may be fit into the framework of the log-theta-lattice developed in $\S 1$.

In the following discussion, we assume that we have been given initial $\Theta$-data as in [IUTchI], Definition 3.1. Let ${ }^{\dagger} \mathcal{H} \mathcal{T}^{\Theta^{ \pm \text {ell }} \mathrm{NF}}$ be a $\Theta^{ \pm \text {ell }} N F$-Hodge theater [relative to the given initial $\Theta$-data - cf. [IUTchI], Definition 6.13, (i)] and

$$
\left\{{ }^{n, m} \mathcal{H} \mathcal{T}^{\Theta^{ \pm \mathrm{ell}} \mathrm{NF}}\right\}_{n, m \in \mathbb{Z}}
$$

a collection of distinct $\Theta^{ \pm \text {ell }} N F$-Hodge theaters [relative to the given initial $\Theta$-data] indexed by pairs of integers, which we think of as arising from a Gaussian log-thetalattice, as in Definition 1.4. We begin by reviewing the theory of theta monoids developed in [IUTchII].

Proposition 2.1. (Vertical Coricity and Kummer Theory of Theta Monoids) We maintain the notation introduced above. Also, we shall use the notation Aut $_{\mathcal{F}}(-)$ to denote the group of automorphisms of the $\mathcal{F}^{\Vdash}$-prime-strip in parentheses. Then:

(i) (Vertically Coric Theta Monoids) In the notation of [IUTchII], Corollary 4.5, (iv), (v), there are functorial algorithms in the $\mathcal{D}$ - and $\mathcal{D}^{\vdash}$-prime-strips ${ }^{\dagger} \mathfrak{D}_{>},{ }^{\dagger} \mathfrak{D}_{>}^{\vdash}$ associated to the $\Theta^{ \pm \mathrm{ell}} N F$-Hodge theater ${ }^{\dagger} \mathcal{H} \mathcal{T}^{\Theta^{ \pm \mathrm{ell}} \mathrm{NF}}$ for constructing collections of data indexed by $\underline{\mathbb{V}}$

$$
\underline{\mathbb{V}} \ni \underline{v} \mapsto \Psi_{\mathrm{env}}\left({ }^{\dagger} \mathfrak{D}_{>}\right)_{\underline{v}} ; \quad \underline{\mathbb{V}} \ni \underline{v} \mapsto \infty \Psi_{\mathrm{env}}\left({ }^{\dagger} \mathfrak{D}_{>}\right)_{\underline{v}}
$$

as well as a global realified Frobenioid

$$
\mathcal{D}_{\text {env }}^{\Vdash}\left({ }^{\dagger} \mathfrak{D}_{>}^{\vdash}\right)
$$

equipped with a bijection Prime $\left(\mathcal{D}_{\text {env }}^{\Vdash}\left({ }^{\dagger} \mathfrak{D}_{>}^{\vdash}\right)\right) \stackrel{\sim}{\rightarrow} \underline{\mathbb{V}}$ and corresponding local isomorphisms, for each $\underline{v} \in \underline{\mathbb{V}}$, as described in detail in [IUTchII], Corollary 4.5, (v). In particular, each isomorphism of the full poly-isomorphism induced [cf. Theorem 1.5, (i)] by a vertical arrow of the Gaussian log-theta-lattice under consideration induces a compatible collection of isomorphisms

$$
\begin{gathered}
\Psi_{\text {env }}\left({ }^{n, m} \mathfrak{D}_{>}\right) \stackrel{\sim}{\rightarrow} \Psi_{\text {env }}\left({ }^{n, m+1} \mathfrak{D}_{>}\right) ; \quad \infty \Psi_{\text {env }}\left({ }^{n, m} \mathfrak{D}_{>}\right) \stackrel{\sim}{\rightarrow} \infty \Psi_{\text {env }}\left({ }^{n, m+1} \mathfrak{D}_{>}\right) \\
\mathcal{D}_{\text {env }}^{\Vdash}\left(n, m \mathfrak{D}_{>}^{\vdash}\right) \stackrel{\sim}{\rightarrow} \mathcal{D}_{\text {env }}^{\Vdash}\left(n, m+1 \mathfrak{D}_{>}^{\vdash}\right)
\end{gathered}
$$

- where the final isomorphism of Frobenioids is compatible with the respective bijections involving "Prime(-)", as well as with the respective local isomorphisms for each $\underline{v} \in \underline{\mathbb{V}}$. 
(ii) (Kummer Isomorphisms) In the notation of [IUTchII], Corollary 4.6, (iv), (v), there are functorial algorithms in the $\Theta^{ \pm \mathrm{ell}} N F$-Hodge theater ${ }^{\dagger} \mathcal{H} \mathcal{T}^{\Theta^{ \pm \mathrm{ell}} \mathrm{NF}}$ for constructing collections of data indexed by $\underline{\mathbb{V}}$

$$
\underline{\mathbb{V}} \ni \underline{v} \mapsto \Psi_{\mathcal{F}_{\text {env }}}\left({ }^{\dagger} \mathcal{H} \mathcal{T}^{\Theta}\right)_{\underline{v}} ; \quad \underline{\mathbb{V}} \ni \underline{v} \mapsto \infty \Psi_{\mathcal{F}_{\text {env }}}\left({ }^{\dagger} \mathcal{H} \mathcal{T}^{\Theta}\right)_{\underline{v}}
$$

as well as a global realified Frobenioid

$$
\mathcal{C}_{\mathrm{env}}^{\Vdash}\left({ }^{\dagger} \mathcal{H} \mathcal{T}^{\Theta}\right)
$$

equipped with a bijection $\operatorname{Prime}\left(\mathcal{C}_{\mathrm{env}}^{\Vdash}\left({ }^{\dagger} \mathcal{H} \mathcal{T}^{\Theta}\right)\right) \stackrel{\sim}{\rightarrow} \underline{\mathbb{V}}$ and corresponding local isomorphisms, for each $\underline{v} \in \underline{\mathbb{V}}$, as described in detail in [IUTchII], Corollary 4.6, (v). Moreover, there are functorial algorithms in ${ }^{\dagger} \mathcal{H} \mathcal{T}^{\Theta^{ \pm e l l} \mathrm{NF}}$ for constructing Kummer isomorphisms

$$
\begin{aligned}
\Psi_{\mathcal{F}_{\text {env }}}\left({ }^{\dagger} \mathcal{H} \mathcal{T}^{\Theta}\right) \stackrel{\sim}{\rightarrow} & \Psi_{\text {env }}\left({ }^{\dagger} \mathfrak{D}_{>}\right) ; \quad \infty \Psi_{\mathcal{F}_{\text {env }}}\left({ }^{\dagger} \mathcal{H} \mathcal{T}^{\Theta}\right) \stackrel{\sim}{\rightarrow}{ }_{\infty} \Psi_{\text {env }}\left({ }^{\dagger} \mathfrak{D}_{>}\right) \\
& \mathcal{C}_{\text {env }}^{\Vdash}\left({ }^{\dagger} \mathcal{H} \mathcal{T}^{\Theta}\right) \stackrel{\sim}{\rightarrow} \mathcal{D}_{\text {env }}^{\Vdash}\left({ }^{\dagger} \mathfrak{D}_{>}^{\vdash}\right)
\end{aligned}
$$

- where the final isomorphism of Frobenioids is compatible with the respective bijections involving "Prime(-)", as well as with the respective local isomorphisms for each $\underline{v} \in \mathbb{V}$ - with the data discussed in (i) [cf. [IUTchII], Corollary 4.6, (iv), (v)]. Finally, the collection of data $\Psi_{\mathrm{env}}\left({ }^{\dagger} \mathfrak{D}_{>}\right)$gives rise, in a natural fashion, to an $\mathcal{F}^{\vdash}$-prime-strip $\mathfrak{F}_{\text {env }}^{-}\left({ }^{\dagger} \mathfrak{D}_{>}\right)$[cf. the $\mathcal{F}^{\vdash}$-prime-strip " $\mathfrak{F}_{\text {env }}^{\vdash}$ " of [IUTchII], Corollary 4.10, (ii)]; the global realified Frobenioid $\mathcal{D}_{\text {env }}^{\Vdash}\left({ }^{\dagger} \mathfrak{D}_{>}^{\vdash}\right)$, equipped with the bijection Prime $\left(\mathcal{D}_{\text {env }}^{\Vdash}\left({ }^{\dagger} \mathfrak{D}_{>}^{\vdash}\right)\right) \stackrel{\sim}{\rightarrow} \underline{\mathbb{V}}$ and corresponding local isomorphisms, for each $\underline{v} \in \underline{\mathbb{V}}$, reviewed in (i), together with the $\mathcal{F}^{\vdash}$-prime-strip $\mathfrak{F}_{\text {env }}^{\vdash}\left({ }^{\dagger} \mathfrak{D}_{>}\right)$, determine an $\mathcal{F}^{\Vdash}$-prime-strip $\mathfrak{F}_{\text {env }}^{\Vdash}\left({ }^{\dagger} \mathfrak{D}_{>}\right)$[cf. the $\mathcal{F}^{\Vdash}$-prime-strip “ $\mathfrak{F}_{\text {env }}^{\Vdash}$ " of [IUTchII], Corollary 4.10, (ii)]. In particular, the first and third Kummer isomorphisms of the above display may be interpreted as [compatible] isomorphisms

$$
{ }^{\dagger} \mathfrak{F}_{\text {env }}^{\vdash} \stackrel{\sim}{\rightarrow} \mathfrak{F}_{\text {env }}^{\vdash}\left({ }^{\dagger} \mathfrak{D}_{>}\right) ; \quad{ }^{\dagger} \mathfrak{F}_{\text {env }}^{\vdash} \stackrel{\sim}{\rightarrow} \mathfrak{F}_{\text {env }}^{\vdash}\left({ }^{\dagger} \mathfrak{D}_{>}\right)
$$

of $\mathcal{F}^{\vdash}$-, $\mathcal{F}^{\Vdash}$-prime-strips.

(iii) (Kummer Theory at Bad Primes) The portion at $\underline{v} \in \underline{\mathbb{V}}^{\text {bad }}$ of the Kummer isomorphisms of (ii) is obtained by composing the Kummer isomorphisms of [IUTchII], Proposition 3.3, (i) - which, we recall, were defined by forming Kummer classes in the context of mono-theta environments that arise from tempered Frobenioids - with the isomorphisms on cohomology classes induced [cf. the upper left-hand portion of the first display of [IUTchII], Proposition 3.4, (i)] by the full poly-isomorphism of projective systems of mono-theta environments $" \mathbb{M}_{*}^{\Theta}\left({ }^{\dagger} \mathcal{D}_{>, \underline{v}}\right) \stackrel{\sim}{\rightarrow} \mathbb{M}_{*}^{\Theta}\left(\underline{\underline{\underline{\mathcal{F}}}}_{v}\right)$ " [cf. [IUTchII], Proposition 3.4; [IUTchII], Remark 4.2.1, (iv)] between projective systems of mono-theta environments that arise from tempered Frobenioids [i.e., " $\underline{\underline{\underline{\mathcal{F}}}} \underline{\underline{v}}$ "] and projective systems of mono-theta environments that arise from the tempered fundamental group [i.e., " $\mathcal{D}_{>, v}$ "] cf. the left-hand portion of the third display of [IUTchII], Corollary 3.6, (ii), in the context of the discussion of Remark 3.6.2, (i). Here, each "isomorphism on cohomology classes" is induced by the isomorphism on exterior cyclotomes

$$
\Pi_{\mu}\left(\mathbb{M}_{*}^{\Theta}\left({ }^{\dagger} \mathcal{D}_{>, \underline{v}}\right)\right) \stackrel{\sim}{\rightarrow} \Pi_{\boldsymbol{\mu}}\left(\mathbb{M}_{*}^{\Theta}\left(\underline{\underline{F}}_{\underline{\underline{\mathcal{F}}}}\right)\right)
$$


determined by each of the isomorphisms that constitutes the full poly-isomorphism of projective systems of mono-theta environments discussed above. In particular, the composite map

$$
\Pi_{\boldsymbol{\mu}}\left(\mathbb{M}_{*}^{\Theta}\left({ }^{\dagger} \mathcal{D}_{>, \underline{v}}\right)\right) \otimes \mathbb{Q} / \mathbb{Z} \quad \rightarrow \quad\left(\Psi_{\dagger \mathcal{F}_{\underline{v}}^{\Theta}}\right)^{\times \mu}
$$

obtained by composing the result of applying $" \otimes \mathbb{Q} / \mathbb{Z} "$ to this isomorphism on exterior cyclotomes with the natural inclusion

$$
\Pi_{\boldsymbol{\mu}}\left(\mathbb{M}_{*}^{\Theta}\left(\underline{\underline{\underline{\mathcal{F}}}}^{\dagger}\right)\right) \otimes \mathbb{Q} / \mathbb{Z} \quad \hookrightarrow \quad\left(\Psi_{\dagger \mathcal{F}_{\underline{\underline{v}}}^{\Theta}}\right)^{\times}
$$

[cf. the notation of [IUTchII], Proposition 3.4, (i); the description given in [IUTchII], Proposition 1.3, (i), of the exterior cyclotome of a mono-theta environment that arises from a tempered Frobenioid] and the natural projection $\left(\Psi_{\dagger \mathcal{F}_{\underline{v}}^{\Theta}}\right)^{\times} \rightarrow\left(\Psi_{\dagger \mathcal{F}_{\underline{v}}^{\Theta}}\right)^{\times \boldsymbol{\mu}}$ is equal to the zero map.

(iv) (Kummer Theory at Good Nonarchimedean Primes) The unit portion at $\underline{v} \in \underline{\mathbb{V}}^{\text {good }} \cap \underline{\mathbb{V}}^{\text {non }}$ of the Kummer isomorphisms of (ii) is obtained [cf. [IUTchII], Proposition 4.2, (iv)] as the unit portion of a "labeled version" of the isomorphism of topological monoids equipped with a topological group action - i.e., in the language of [AbsTopIII], Definition 3.1, (ii), the isomorphism of "MLF-Galois TM-pairs" - discussed in [IUTchII], Proposition 4.2, (i) [cf. also [IUTchII], Remark 1.11.1, (i), (a); [AbsTopIII], Proposition 3.2, (iv)]. In particular, the portion at $\underline{v} \in \underline{\mathbb{V}}^{\text {good }} \cap \underline{\mathbb{V}}^{\text {non }}$ of the $\operatorname{Aut}_{\mathcal{F}^{\Vdash}}\left({ }^{\dagger} \mathfrak{F}_{\text {env }}^{\vdash}\right)$-orbit of the second isomorphism of the final display of (ii) may be obtained as a "labeled version" of the "Kummer poly-isomorphism of semi-simplifications" given in the final display of [IUTchII], Proposition 4.2, (ii).

(v) (Kummer Theory at Good Archimedean Primes) The unit portion at $\underline{v} \in \underline{\mathbb{V}}^{\text {good }} \cap \underline{\mathbb{V}}^{\text {arc }}$ of the Kummer isomorphisms of (ii) is obtained [cf. [IUTchII], Proposition 4.4, (iv)] as the unit portion of a "labeled version" of the isomorphism of topological monoids discussed in [IUTchII], Proposition 4.4, (i). In particular, the portion at $\underline{v} \in \underline{\mathbb{V}}^{\text {good }} \cap \underline{\mathbb{V}}^{\text {arc }}$ of the Aut $\operatorname{\mathcal {F}}^{\Vdash}\left({ }^{\dagger} \mathfrak{F}_{\text {env }}^{\Vdash}\right)$-orbit of the second isomorphism of the final display of (ii) may be obtained as a "labeled version" of the "Kummer poly-isomorphism of semi-simplifications" given in the final display of [IUTchII], Proposition 4.4, (ii) [cf. also [IUTchII], Remark 4.6.1].

(vi) (Compatibility with Constant Monoids) The definition of the unit portion of the theta monoids involved [cf. [IUTchII], Corollary 4.10, (iv)] gives rise to natural isomorphisms

$$
{ }^{\dagger} \mathfrak{F}_{\triangle}^{\vdash \times} \stackrel{\sim}{\rightarrow}{ }^{\dagger} \mathfrak{F}_{\text {env }}^{\vdash \times} ; \quad \mathfrak{F}_{\triangle}^{\vdash \times}\left({ }^{\dagger} \mathfrak{D}_{\triangle}^{\vdash}\right) \stackrel{\sim}{\rightarrow} \mathfrak{F}_{\text {env }}^{\vdash \times}\left({ }^{\dagger} \mathfrak{D}_{>}\right)
$$

- i.e., where the morphism induced on $\mathcal{F}^{\vdash \times \boldsymbol{\mu}}$-prime-strips by the first displayed isomorphism is precisely the isomorphism of the first display of [IUTchII], Corollary 4.10, (iv) - of the respective associated $\mathcal{F}^{-\times}$-prime-strips [cf. the notation of Theorem 1.5, (iii), where the label " $n, m$ " is replaced by the label "†"]. Moreover, these natural isomorphisms are compatible with the Kummer isomorphisms of (ii) above and Theorem 1.5, (iii). 
Proof. The various assertions of Proposition 2.1 follow immediately from the definitions and the references quoted in the statements of these assertions.

Remark 2.1.1. The theory of mono-theta environments [cf. Proposition 2.1, (iii)] will play a crucial role in the theory of the present $\S 2$ [cf. Theorem 2.2, (ii); Corollary 2.3, (iv), below] in the passage from Frobenius-like to étale-like structures [cf. Remark 1.5.4, (i), (a)] at bad primes. In particular, the various rigidity properties of mono-theta environments established in [EtTh] play a fundamental role in ensuring that the resulting "Kummer-detachment indeterminacies" [cf. the discussion of Remark 1.5.4, (i)] are sufficiently mild so as to allow the establishment of the various reconstruction algorithms of interest. For this reason, we pause to review the main properties of mono-theta environments established in [EtTh] [cf. [EtTh], Introduction] — namely,
(a) cyclotomic rigidity
(b) discrete rigidity
(c) constant multiple rigidity
(d) isomorphism class compatibility
(e) Frobenioid structure compatibility

- and the roles played by these main properties in the theory of the present series of papers. Here, we remark that "isomorphism class compatibility" [i.e., (d)] refers to compatibility with the convention that various objects of the tempered Frobenioids [and their associated base categories] under consideration are known only up to isomorphism [cf. [EtTh], Corollary 5.12; [EtTh], Remarks 5.12.1, 5.12.2]. In the Introduction to [EtTh], instead of referring to (d) in this form, we referred to the property of compatibility with the topology of the tempered fundamental group. In fact, however, this compatibility with the topology of the tempered fundamental group is a consequence of (d) [cf. [EtTh], Remarks 5.12.1, 5.12.2]. On the other hand, from the point of view of the present series of papers, the essential property of interest in this context is best understood as being the property (d).

(i) First, we recall that the significance, in the context of the theory of the present series of papers, of the compatibility with the Frobenioid structure of the tempered Frobenioids under consideration [i.e., (e)] — i.e., in particular, with the monoidal portion, equipped its natural Galois action, of these Frobenioids - lies in the role played by this "Frobenius-like" monoidal portion in performing constructions - such as, for instance, the construction of the $\mathfrak{l o g}_{-}, \Theta_{-}, \Theta^{\times \boldsymbol{\mu}_{-}}$, or $\Theta_{\text {gau }}^{\times \boldsymbol{\mu}_{\text {-links }}}$ - that are "not bound to conventional scheme theory", but may be related, via Kummer theory, to various étale-like structures [cf. the discussions of Remark 1.5.4, (i); [IUTchII], Remark 3.6.2, (ii); [IUTchII], Remark 3.6.4, (ii), (v)].

(ii) Next, we consider isomorphism class compatibility [i.e., (d)]. As discussed above, this compatibility corresponds to regarding each of the various objects of the tempered Frobenioids [and their associated base categories] under consideration as being known only up to isomorphism [cf. [EtTh], Corollary 5.12; [EtTh], Remarks 5.12.1, 5.12.2]. As discussed in [IUTchII], Remark 3.6.4, (i), the significance of this property (d) in the context of the present series of papers lies in the fact that - unlike the case with the projective systems constituted by Kummer towers constructed from $N$-th power morphisms, which are compatible with only the 
multiplicative, but not the additive structures of the $p_{\underline{v}}$-adic local fields involved each individual object in such a Kummer tower corresponds to a single field [i.e., as opposed to a projective system of multiplicative groups of fields]. This field/ring structure is necessary in order to apply the theory of the log-link developed in $\S 1$ - cf. the vertical coricity discussed in Proposition 2.1, (i). Note, moreover, that, unlike the $\mathfrak{l o g}_{-}, \Theta_{-}, \Theta^{\times \boldsymbol{\mu}_{-}}$, or $\Theta_{\text {gau }}^{\times \boldsymbol{\mu}}$-links, the $N$-th power morphisms that appear in a Kummer tower are "algebraic", i.e., scheme-theoretic, hence compatible with the conventional scheme-theoretic theory of the étale [or tempered] fundamental group. In particular, since the tempered Frobenioids under consideration may be constructed from such scheme-theoretic categories, the fundamental groups on either side of such an $N$-th power morphism may be related up to an indeterminacy arising from an inner automorphism of the tempered fundamental group [i.e., the "fundamental group" of the base category] under consideration - cf. the discussion of [IUTchII], Remark 3.6.3, (ii). On the other hand, the objects that appear in these Kummer towers necessarily arise from nontrivial line bundles [indeed, line bundles all of whose positive tensor powers are nontrivial!] on tempered coverings of a Tate curve - cf. the constructions underlying the Frobenioid-theoretic version of the mono-theta environment [cf. [EtTh], Proposition 1.1; [EtTh], Lemma 5.9]; the crucial role played by the commutator " $[-,-]$ " in the theory of cyclotomic rigidity [i.e., (a)] reviewed in (iv) below. In particular, the extraction of various $N$-th roots in a Kummer tower necessarily leads to mutually non-isomorphic line bundles, i.e., mutually non-isomorphic objects in the Kummer tower. From the point of view of reconstruction algorithms, such non-isomorphic objects may be naturally - i.e., algorithmically - related to another only via indeterminate isomorphisms [cf., (d)!]. This point of view is precisely the starting point of the discussion of - for instance, "constant multiple indeterminacy" in — [EtTh], Remarks 5.12.2, 5.12 .3 .

(iii) Next, we recall that the significance of constant multiple rigidity [i.e., (c)] in the context of the present series of papers lies in the construction of the canonical splittings of theta monoids via restriction to the zero section discussed, for instance, in [IUTchII], Corollary 1.12, (ii); [IUTchII], Proposition 3.3, (i); [IUTchII], Remark 1.12.2, (iv) [cf. also Remark 1.2.3, (i), of the present paper].

(iv) Next, we review the significance of cyclotomic rigidity [i.e., (a)] in the context of the present series of papers. First, we recall that this cyclotomic rigidity is essentially a consequence of the nondegenerate nature of the commutator " $[-,-]$ " of the theta groups involved [cf. the discussion of [EtTh], Introduction; [EtTh], Remark 2.19.2]. Put another way, since this commutator is quadratic in nature, one may think of this nondegenerate nature of the commutator as a statement to the effect that "the degree of the commutator is precisely 2". At a more concrete level, the cyclotomic rigidity arising from a mono-theta environment consists of a certain specific isomorphism between the interior and exterior cyclotomes [cf. the discussion of [IUTchII], Definition 1.1, (ii)]. Put another way, one may think of this cyclotomic rigidity isomorphism as a sort of rigidification of a certain "projective line of cyclotomes", i.e., the projectivization of the direct sum of the interior and exterior cyclotomes [cf. the computations that underlie [EtTh], Proposition 2.12]. In particular, this rigidification is fundamentally nonlinear in nature. Indeed, if one attempts to compose it with an $N$-th power morphism, then one is obliged to sacrifice constant multiple rigidity [i.e., (c)] — cf. the discussion of [EtTh], 
Remark 5.12.3. That is to say, the distinguished nature of the "first power" of the cyclotomic rigidity isomorphism is an important theme in the theory of [EtTh] [cf. the discussion of [EtTh], Remark 5.12.5; [IUTchII], Remark 3.6.4, (iii), (iv)]. The multiradiality of mono-theta-theoretic cyclotomic rigidity [cf. [IUTchII], Corollary 1.10] — which lies in stark contrast with the indeterminacies that arise when one attempts to give a multiradial formulation [cf. [IUTchII], Corollary 1.11; the discussion of [IUTchII], Remark 1.11.3] of the more classical "MLF-Galois pair cyclotomic rigidity" arising from local class field theory - will play a central role in the theory of the present $\S 2$ [cf. Theorem 2.2, Corollary 2.3 below].

(v) Finally, we review the significance of discrete rigidity [i.e., (b)] in the context of the present series of papers. First, we recall that, at a technical level, whereas cyclotomic rigidity may be regarded [cf. the discussion of (iv)] as a consequence of the fact that "the degree of the commutator is precisely 2", discrete rigidity may be regarded as a consequence of the fact that "the degree of the commutator is $\leq 2$ " [cf. the statements and proofs of [EtTh], Proposition 2.14, (ii), (iii)]. At a more concrete level, discrete rigidity assures one that one may restrict one's attentions to $\mathbb{Z}$-multiples/powers — as opposed to $\widehat{\mathbb{Z}}$-multiples/powers — of divisors, line bundles, and rational functions [such as, for instance, the q-parameter!] on the tempered coverings of a Tate curve that occur in the theory of [EtTh] [cf. [EtTh], Remark 2.19.4]. This prompts the following question:

Can one develop a theory of $\widehat{\mathbb{Z}}$-divisors/line bundles/rational functions in, for instance, a parallel fashion to the way in which one considers perfections and realifications of Frobenioids in the theory of [FrdI]?

As far as the author can see at the time of writing, the answer to this question is "no". Indeed, unlike the case with $\mathbb{Q}$ or $\mathbb{R}$, there is no notion of positivity [or negativity] in $\widehat{\mathbb{Z}}$. For instance, $-1 \in \widehat{\mathbb{Z}}$ may be obtained as a limit of positive integers. In particular, if one had a theory of $\widehat{\mathbb{Z}}$-divisors/line bundles/rational functions, then such a theory would necessarily require one to "confuse" positive [i.e., effective] and negative divisors, hence to work birationally. But to work birationally means, in particular, that one must sacrifice the conventional structure of isomorphisms [e.g., automorphisms] between line bundles - which plays an indispensable role, for instance, in the constructions underlying the Frobenioid-theoretic version of the mono-theta environment [cf. [EtTh], Proposition 1.1; [EtTh], Lemma 5.9; the crucial role played by the commutator " $[-,-]$ " in the theory of cyclotomic rigidity [i.e., (a)] reviewed in (iv) above].

\section{Remark 2.1.2.}

(i) In the context of the discussion of Remark 2.1.1, (v), it is of interest to recall [cf. [IUTchII], Remark 4.5.3, (iii); [IUTchII], Remark 4.11.2, (iii)] that the essential role played, in the context of the $\mathbb{F}_{l}^{\rtimes \pm}$-symmetry, by the "global bookkeeping operations" involving the labels of the evaluation points gives rise, in light of the profinite nature of the global étale fundamental groups involved, to a situation in which one must apply the "complements on tempered coverings" developed in [IUTchI], §2. That is to say, in the notation of the discussion given in [IUTchII], 
Remark 2.1.1, (i), of the various tempered coverings that occur at $\underline{v} \in \underline{\mathbb{V}}^{\text {bad }}$, these "complements on tempered coverings" are applied precisely so as to allow one to restrict one's attention to the [discrete! $\mathbb{Z}$-conjugates - i.e., as opposed to [profinite! $\underline{\mathbb{Z}}$-conjugates [where we write $\underline{\mathbb{Z}}$ for the profinite completion of $\underline{\mathbb{Z}}$ ] - of the theta functions involved. In particular, although such "evaluation-related issues", which will become relevant in the context of the theory of $\S 3$ below, do not play a role in the theory of the present $\S 2$, the role played by the theory of [IUTchI], $\S 2$, in the theory of the present series of papers may also be thought of as a sort of "discrete rigidity" - which we shall refer to as "evaluation discrete rigidity" - i.e., a sort of rigidity that is concerned with similar issues to the issues discussed in the case of "mono-theta-theoretic discrete rigidity" in Remark 2.1.1, (v), above.

(ii) Next, let us suppose that we are in the situation discussed in [IUTchII], Proposition 2.1. Fix $\underline{v} \in \underline{\mathbb{V}}^{\text {bad }}$. Write $\Pi \stackrel{\text { def }}{=} \Pi_{\underline{v}}$; $\widehat{\Pi}$ for the profinite completion of $\Pi$. Thus, we have natural surjections $\Pi \rightarrow l \cdot \underline{\mathbb{Z}}(\underline{\subseteq} \underline{\mathbb{Z}}), \widehat{\Pi} \rightarrow l \cdot \underline{\mathbb{Z}}(\underline{\mathbb{\mathbb { Z }}})$. Write $\Pi^{\dagger} \stackrel{\text { def }}{=} \widehat{\Pi} \times_{\widehat{\mathbb{Z}}} \underline{\mathbb{Z}} \subseteq \widehat{\Pi}$. Next, we observe that from the point of view of the evaluation points, the evaluation discrete rigidity discussed in (i) corresponds to the issue of whether, relative to some arbitrarily chosen basepoint, the coordinates of the evaluation point lie $\in \underline{\mathbb{Z}}$ or $\in \underline{\mathbb{Z}}[\mathrm{cf}$. the discussion of the "torsor over $\underline{\mathbb{Z}}$ " in [IUTchII], Remark 2.1.1, (i)]. Thus, if one is only concerned with the issue of arranging for these coordinates to lie $\in \underline{\mathbb{Z}}$, then one is led to pose the following question:

Is it possible to simply use the "partially tempered fundamental group" $\Pi^{\dagger}$ instead of the "full" tempered fundamental group $\Pi$ in the theory of the present series of papers?

The answer to this question is "no". One way to see this is to consider the [easily verified] natural isomorphism

$$
N_{\widehat{\Pi}}\left(\Pi^{\dagger}\right) / \Pi^{\dagger} \stackrel{\sim}{\rightarrow} \underline{\mathbb{Z}} / \underline{\mathbb{Z}}
$$

involving the normalizer $N_{\widehat{\Pi}}\left(\Pi^{\dagger}\right)$ of $\Pi^{\dagger}$ in $\widehat{\Pi}$. One consequence of this isomorphism is that - unlike the tempered fundamental group $\Pi$ [cf., e.g., [SemiAnbd], Theorems $6.6,6.8]$ - the topological group $\Pi^{\dagger}$ fails to satisfy various fundamental absolute anabelian properties which play a crucial role in the theory of [EtTh], as well as in the present series of papers [cf., e.g., the theory of [IUTchII], §2]. At a more concrete level, unlike the case with the tempered fundamental group $\Pi$, the profinite conjugacy indeterminacies that act on $\Pi^{\dagger}$ give rise to $\underline{\mathbb{Z}}$-translation indeterminacies acting on the coordinates of the evaluation points involved. That is to say, in the case of $\Pi$, such $\underline{\mathbb{Z}}$-translation indeterminacies are avoided precisely by applying the "complements on tempered coverings" developed in [IUTchI], §2 - i.e., in a word, as a consequence of the "highly anabelian nature" of the [full!] tempered fundamental group $\Pi$.

Theorem 2.2. (Kummer-compatible Multiradiality of Theta Monoids) Fix a collection of initial $\Theta$-data

$$
\left(\bar{F} / F, X_{F}, l, \underline{C}_{K}, \underline{\mathbb{V}}, \underline{\epsilon}\right)
$$


as in [IUTchI], Definition 3.1. Let ${ }^{\dagger} \mathcal{H} \mathcal{T}^{\Theta^{ \pm \mathrm{ell}} \mathrm{NF}}$ be a $\Theta^{ \pm \mathrm{ell}} \mathbf{N F}-$ Hodge theater [relative to the given initial $\Theta$-data - cf. [IUTchI], Definition 6.13, (i)]. For $\square \in\{\Vdash, \Vdash \times \boldsymbol{\mu}, \vdash \times \boldsymbol{\mu}\}$, write $\operatorname{Aut}_{\mathcal{F} \square}(-)$ for the group of automorphisms of the $\mathcal{F}^{\square}$-prime-strip in parentheses.

(i) (Automorphisms of Prime-strips) The natural functors determined by assigning to an $\mathcal{F}^{\Vdash}$-prime-strip the associated $\mathcal{F}^{\Vdash} \triangleright \times \boldsymbol{\mu}_{\text {- }}$ and $\mathcal{F}^{\vdash \times \boldsymbol{\mu}}$-prime-strips [cf. [IUTchII], Definition 4.9, (vi), (vii), (viii)] and then composing with the natural isomorphisms of Proposition 2.1, (vi), determine natural homomorphisms

$$
\begin{aligned}
& \operatorname{Aut}_{\mathcal{F} \Vdash}\left(\mathfrak{F}_{\text {env }}^{\Vdash}\left({ }^{\dagger} \mathfrak{D}_{>}\right)\right) \rightarrow \operatorname{Aut}_{\mathcal{F} \Vdash \triangleright \times \mu}\left(\mathfrak{F}_{\text {env }}^{\Vdash \bullet} \times \boldsymbol{\mu}\left({ }^{\dagger} \mathfrak{D}_{>}\right)\right) \rightarrow \operatorname{Aut}_{\mathcal{F} \vdash \times \mu}\left(\mathfrak{F}_{\triangle}^{\vdash \times \mu}\left({ }^{\dagger} \mathfrak{D}_{\triangle}^{\vdash}\right)\right) \\
& \operatorname{Aut}_{\mathcal{F} \Vdash}\left({ }^{\dagger} \mathfrak{F}_{\text {env }}^{\Vdash}\right) \rightarrow \operatorname{Aut}_{\mathcal{F} \Vdash \triangleright \times \mu}\left({ }^{\ddagger} \mathfrak{F}_{\text {env }}^{\Vdash \bullet} \times \boldsymbol{\mu}\right) \rightarrow \operatorname{Aut}_{\mathcal{F} \vdash \times \mu}\left({ }^{\dagger} \mathfrak{F}_{\triangle}^{\vdash} \times \boldsymbol{\mu}\right)
\end{aligned}
$$

- where the second arrows in each line are surjections - that are compatible with the Kummer isomorphisms of Proposition 2.1, (ii), and Theorem 1.5, (iii) [cf. the final portions of Proposition 2.1, (iv), (v), (vi)]. Write

(ii) (Kummer Aspects of Multiradiality at Bad Primes) Let $\underline{v} \in \underline{\mathbb{V}}^{\text {bad }}$.

$$
\infty \Psi_{\text {env }}^{\perp}\left({ }^{\dagger} \mathfrak{D}_{>}\right)_{\underline{v}} \subseteq \infty \Psi_{\mathrm{env}}\left({ }^{\dagger} \mathfrak{D}_{>}\right)_{\underline{v}} ; \quad \infty \Psi_{\mathcal{F}_{\text {env }}}^{\perp}\left({ }^{\dagger} \mathcal{H} \mathcal{T}^{\Theta}\right)_{\underline{v}} \subseteq \infty \Psi_{\mathcal{F}_{\text {env }}}\left({ }^{\dagger} \mathcal{H} \mathcal{T}^{\Theta}\right)_{\underline{v}}
$$

for the submonoids corresponding to the respective splittings [cf. [IUTchII], Corollaries 3.5, (iii); 3.6, (iii)], i.e., the submonoids generated by " $\infty \underline{\theta}_{\text {env }}^{\iota}\left(\mathbb{M}_{*}^{\Theta}\right)$ " [cf. the notation of [IUTchII], Proposition 3.1, (i)] and the respective torsion subgroups. Now consider the commutative diagram

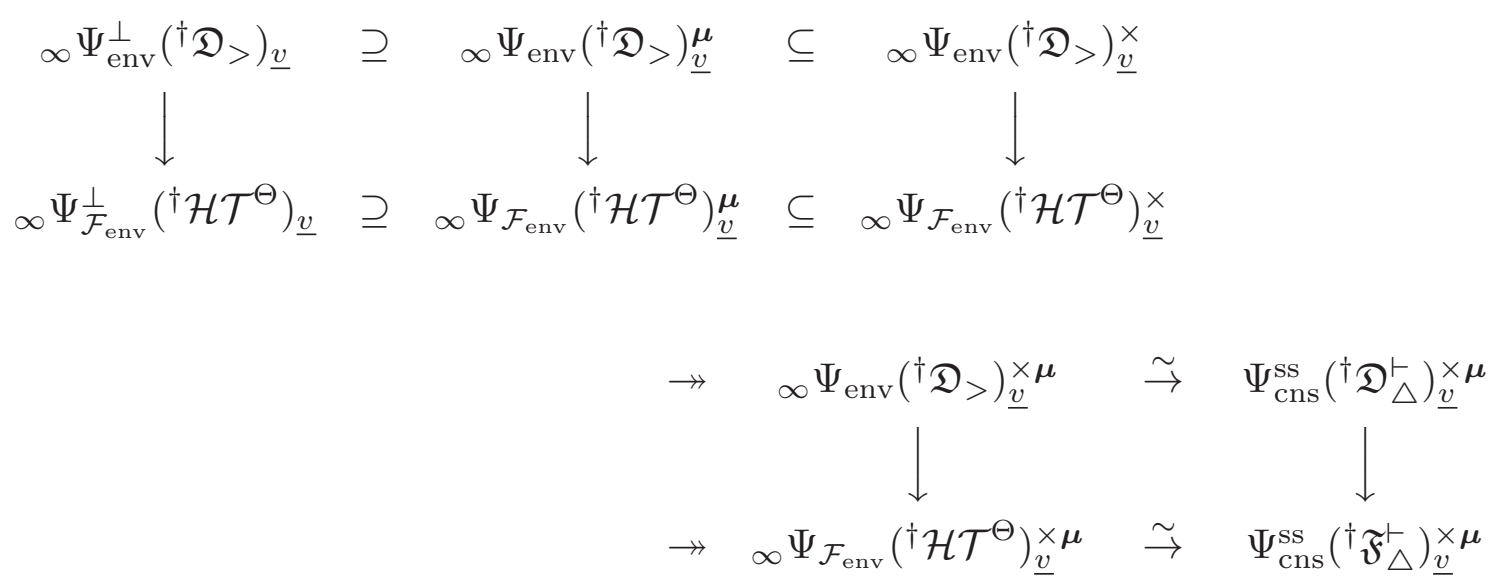

- where the inclusions " $\supseteq$ ", " $\subseteq$ " are the natural inclusions; the surjections " $\rightarrow$ " are the natural surjections; the superscript " $\mu$ " denotes the torsion subgroup; the superscript " $\times$ " denotes the group of units; the superscript " $\times \boldsymbol{\mu}$ " denotes the quotient " $(-)^{\times} /(-)^{\boldsymbol{\mu}}$ "; the first four vertical arrows are the isomorphisms determined by the inverse of the second Kummer isomorphism of the third display of Proposition 2.1, (ii); ${ }^{\dagger} \mathfrak{D}_{\triangle} \triangle$ is as discussed in Theorem 1.5, (iii); ${ }^{\dagger} \mathfrak{F}_{\triangle}{ }^{-}$is as discussed in [IUTchII], Corollary 4.10, (i); the final vertical arrow is the inverse of the Kummer isomorphism determined by the final displayed isomorphism of [IUTchII], Corollary 4.6, (i) [cf. also the isomorphism of the fourth display of [IUTchII], Corollary 4.5, (ii)]; the final upper horizontal arrow is the poly-isomorphism determined by composing the isomorphism determined by the inverse of the natural 
isomorphism of Proposition 2.1, (vi), with the poly-automorphism of $\Psi_{\mathrm{cns}}^{\mathrm{ss}}\left({ }^{\dagger} \mathfrak{D}_{\triangle}^{\vdash}\right)_{\underline{v}}^{\times \mu}$ induced by the full poly-automorphism of the $\mathcal{D}^{\vdash}$-prime-strip ${ }^{\dagger} \mathfrak{D}_{\triangle}^{\vdash}$; the final lower horizontal arrow is the poly-automorphism determined by the condition that the final square be commutative. This commutative diagram is compatible with the various group actions involved relative to the following diagram

$$
\begin{aligned}
& \Pi_{\underline{\underline{X}}}\left(\mathbb{M}_{*}^{\Theta}\left({ }^{\dagger} \mathcal{D}_{>, \underline{v}}\right)\right) \rightarrow G_{\underline{v}}\left(\mathbb{M}_{*}^{\Theta}\left({ }^{\dagger} \mathcal{D}_{>, \underline{v}}\right)\right) \\
&=G_{\underline{v}}\left(\mathbb{M}_{*}^{\Theta}\left({ }^{\dagger} \mathcal{D}_{>, \underline{v}}\right)\right) \stackrel{\sim}{\rightarrow} G_{\underline{\underline{v}}}\left(\mathbb{M}_{*}^{\Theta}\left(\mathbb{M}_{*}^{\Theta}\left(\mathcal{D}_{>, \underline{v}}\right)\right)\right. \\
&\left.\left.{ }^{\dagger} \mathcal{D}_{>, \underline{v}}\right)\right)
\end{aligned}
$$

[cf. the notation of [IUTchII], Proposition 3.1; [IUTchII], Remark 4.2.1, (iv); [IUTchII], Corollary 4.5, (iv)] - where " $\rightarrow$ " denotes the natural surjection; " $\stackrel{\sim}{\rightarrow}$ " denotes the full poly-automorphism of $G_{\underline{v}}\left(\mathbb{M}_{*}^{\Theta}\left({ }^{\dagger} \mathcal{D}_{>, \underline{v}}\right)\right)$. Finally, each of the various composite maps

$$
\infty \Psi_{\mathrm{env}}\left({ }^{\dagger} \mathfrak{D}_{>}\right)_{\underline{v}}^{\mu} \rightarrow \Psi_{\mathrm{cns}}^{\mathrm{ss}}\left({ }^{\dagger} \mathfrak{F}_{\triangle}^{\vdash}\right)_{\underline{v}}^{\times \mu}
$$

is equal to the zero map [cf. ( $\left.b_{\underline{v}}\right)$ below; the final portion of Proposition 2.1, (iii)]. In particular, the identity automorphism on the following objects is compatible, relative to the various natural morphisms involved [cf. the above commutative diagram], with the collection of automorphisms of $\Psi_{\mathrm{cns}}^{\mathrm{ss}}\left({ }^{\dagger} \mathfrak{F}_{\triangle}^{\vdash}\right)_{\underline{v}}^{\times \mu}$ induced by arbitrary automorphisms $\in \operatorname{Aut}_{\mathcal{F}^{\vdash \times \mu}}\left({ }^{\dagger} \mathfrak{F}_{\triangle}^{\vdash \times \mu}\right)$ [cf. [IUTchII], Corollary 1.12, (iii); [IUTchII], Proposition 3.4, (i)]:

$\left(a_{\underline{v}}\right)_{\infty} \Psi_{\mathrm{env}}^{\perp}\left({ }^{\dagger} \mathfrak{D}_{>}\right)_{\underline{v}} \supseteq \Psi_{\mathrm{env}}\left({ }^{\dagger} \mathfrak{D}_{>}\right)_{\underline{\underline{\mu}}}^{\boldsymbol{\mu}} ;$

$\left(b_{\underline{v}}\right) \Pi_{\boldsymbol{\mu}}\left(\mathbb{M}_{*}^{\Theta}\left({ }^{\dagger} \mathcal{D}_{>, \underline{v}}\right)\right) \otimes \mathbb{Q} / \mathbb{Z}[c f$. the discussion of Proposition 2.1, (iii)], relative to the natural isomorphism $\Pi_{\boldsymbol{\mu}}\left(\mathbb{M}_{*}^{\Theta}\left({ }^{\dagger} \mathcal{D}_{>, \underline{v}}\right)\right) \otimes \mathbb{Q} / \mathbb{Z} \stackrel{\sim}{\rightarrow} \infty \Psi_{\mathrm{env}}\left({ }^{\dagger} \mathfrak{D}_{>}\right)_{v}^{\boldsymbol{\mu}}$ of [IUTchII], Remark 1.5.2 [cf. $\left.\left(a_{\underline{v}}\right)\right]$;

$\left(c_{\underline{v}}\right)$ the projective system of mono-theta environments $\mathbb{M}_{*}^{\Theta}\left({ }^{\dagger} \mathcal{D}_{>, \underline{v}}\right)$ [cf. $\left.\left(b_{\underline{v}}\right)\right]$;

$\left(d_{\underline{v}}\right)$ the splittings $\infty \Psi_{\mathrm{env}}^{\perp}\left({ }^{\dagger} \mathfrak{D}_{>}\right)_{\underline{v}} \rightarrow \infty \Psi_{\mathrm{env}}\left({ }^{\dagger} \mathfrak{D}_{>}\right)_{\underline{\boldsymbol{\mu}}}^{\boldsymbol{\mu}}\left[c f . \quad\left(a_{\underline{v}}\right)\right]$ by means of restriction to zero-labeled evaluation points [cf. [IUTchII], Proposition 3.1, (i)].

Proof. The various assertions of Theorem 2.2 follow immediately from the definitions and the references quoted in the statements of these assertions.

Remark 2.2.1. In light of the central importance of Theorem 2.2, (ii), in the theory of the present $\S 2$, we pause to examine the significance of Theorem 2.2, (ii), in more conceptual terms.

(i) In the situation of Theorem 2.2, (ii), let us write [for simplicity] $\Pi_{\underline{v}} \stackrel{\text { def }}{=}$ $\Pi_{\underline{\underline{X}}}\left(\mathbb{M}_{*}^{\Theta}\left({ }^{\dagger} \mathcal{D}_{>, \underline{v}}\right)\right), \Pi_{\boldsymbol{\mu}} \stackrel{\text { def }}{=} \Pi_{\boldsymbol{\mu}}\left(\mathbb{M}_{*}^{\Theta}\left({ }^{\dagger} \mathcal{D}_{>, \underline{v}}\right)\right)\left[\mathrm{cf} .\left(\mathrm{b}_{\underline{v}}\right)\right]$. Also, for simplicity, we write $\left(l \cdot \Delta_{\Theta}\right) \stackrel{\text { def }}{=}\left(l \cdot \Delta_{\Theta}\right)\left(\mathbb{M}_{*}^{\Theta}\left({ }^{\dagger} \mathcal{D}_{>, \underline{v}}\right)\right)[$ cf. [IUTchII], Proposition 1.5, (iii)]. Here, we recall that in fact, $\left(l \cdot \Delta_{\Theta}\right)$ may be thought of as an object constructed from $\Pi_{\underline{v}}$ 
[cf. [IUTchII], Proposition 1.4]. Then the projective system of mono-theta environments $\mathbb{M}_{*}^{\Theta}\left({ }^{\dagger} \mathcal{D}_{>, \underline{v}}\right)$ [cf. $\left(c_{\underline{v}}\right)$ ] may be thought of as a sort of "amalgamation of $\Pi_{\underline{v}}$ and $\Pi_{\mu}$ ", where the amalgamation is such that it allows the reconstruction of the mono-theta-theoretic cyclotomic rigidity isomorphism

$$
\left(l \cdot \Delta_{\Theta}\right) \stackrel{\sim}{\rightarrow} \Pi_{\mu}
$$

[cf. [IUTchII], Proposition 1.5, (iii)] — i.e., not just the $\widehat{\mathbb{Z}}^{\times}$-orbit of this isomorphism!

(ii) Now, in the notation of (i), the Kummer classes $\in \infty_{\infty}^{\perp} \Psi_{\mathrm{env}}^{\perp}\left({ }^{\dagger} \mathfrak{D}_{>}\right)_{\underline{v}}\left[\right.$ cf. $\left.\left(\mathrm{a}_{\underline{v}}\right)\right]$ constituted by the various étale theta functions may be thought of, for an appropriate characteristic open subgroup $H \subseteq \Pi_{\underline{v}}$, as twisted homomorphisms

$$
\left(\Pi_{\underline{v}} \supseteq\right) H \rightarrow \Pi_{\mu}
$$

whose restriction to $\left(l \cdot \Delta_{\Theta}\right)$ coincides with the cyclotomic rigidity isomorphism $\left(l \cdot \Delta_{\Theta}\right) \stackrel{\sim}{\rightarrow} \Pi_{\boldsymbol{\mu}}$ discussed in (i). Then the essential content of Theorem 2.2, (ii), lies in the observation that

since the Kummer-theoretic link between étale-like data and Frobeniuslike data at $\underline{v} \in \underline{\mathbb{V}}^{\text {bad }}$ is established by means of projective systems of mono-theta environments [cf. the discussion of Proposition 2.1, (iii)] - i.e., which do not involve the various monoids " $(-)^{\times \mu}$ "! — the monotheta-theoretic cyclotomic rigidity isomorphism [i.e., not just the $\widehat{\mathbb{Z}}^{\times}$-orbit of this isomorphism!] is immune to the various automorphisms of the monoids " $(-)^{\times \mu}$ " which, from the point of view of the multiradial formulation to be discussed in Corollary 2.3 below, arise from isomorphisms of coric data.

Put another way, this "immunity" may be thought of as a sort of decoupling of the "geometric" [i.e., in the sense of the geometric fundamental group $\Delta_{\underline{v}} \subseteq \Pi_{\underline{v}}$ ] and "base-field-theoretic" [i.e., associated to the local absolute Galois group $\Pi_{\underline{v}} \rightarrow G_{v}$ ] data which allows one to treat the exterior cyclotome $\Pi_{\mu}$ - which, a priori, "looks base-field-theoretic" - as being part of the "geometric" data. From the point of view of the multiradial formulation to be discussed in Corollary 2.3 below [cf. also the discussion of [IUTchII], Remark 1.12.2, (vi)], this decoupling may be thought of as a sort of splitting into purely radial and purely coric components - i.e., with respect to which $\Pi_{\boldsymbol{\mu}}$ is "purely radial", while the various monoids " $(-)^{\times \boldsymbol{\mu}}$ " are "purely coric".

(iii) Note that the immunity to automorphisms of the monoids " $(-)^{\times \mu}$ " discussed in (ii) lies in stark contrast to the $\widehat{\mathbb{Z}}^{\times}$-indeterminacies that arise in the case of the cyclotomic rigidity isomorphisms constructed from MLF-Galois pairs in a fashion that makes essential use of the monoids " $(-)^{\times \boldsymbol{\mu}}$ ", as discussed in [IUTchII], Corollary 1.11; [IUTchII], Remark 1.11.3. In the following discussion, let us write "O ${ }^{\times \mu}$ " for the various monoids " $(-)^{\times \boldsymbol{\mu}}$ " that occur in the situation of Theorem

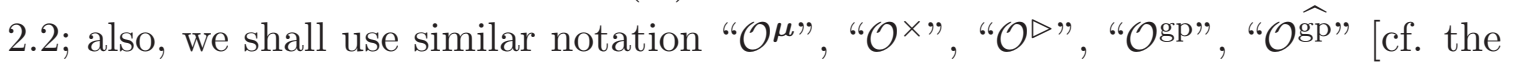


notational conventions of [IUTchII], Example 1.8, (iv), (vii)]. Thus, we have a diagram

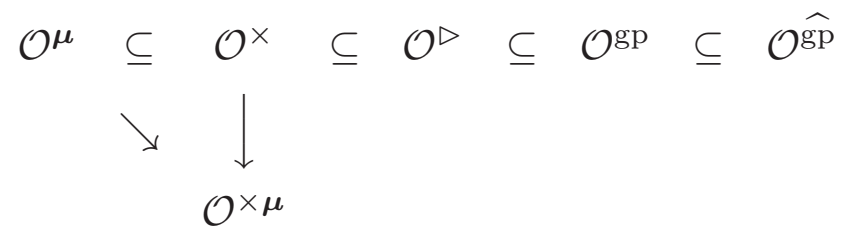

of natural morphisms between monoids equipped with $\Pi_{\underline{v}}$-actions. Relative to this notation, the essential input data for the cyclotomic rigidity isomorphism constructed from an MLF-Galois pair is given by "OO " [cf. [IUTchII], Corollary 1.11, (a)]. On the other hand - unlike the case with $\mathcal{O}^{\boldsymbol{\mu}}-$ a $\widehat{\mathbb{Z}}^{\times}$-indeterminacy acting on $\mathcal{O}^{\times \boldsymbol{\mu}}$ does not lie under an identity action on $\mathcal{O}^{\times}$! That is to say, a $\widehat{\mathbb{Z}}^{\times}$indeterminacy acting on $\mathcal{O}^{\times \boldsymbol{\mu}}$ can only be lifted naturally to $\widehat{\mathbb{Z}}^{\times}$-indeterminacies on $\mathcal{O}^{\times}, \widehat{\mathcal{O}^{\text {gp }}}$ [cf. Fig. 2.1 below; [IUTchII], Corollary 1.11, (a), in the case where one takes " $\Gamma$ " to be $\widehat{\mathbb{Z}}^{\times}$; [IUTchII], Remark 1.11.3, (ii)]. In the presence of such $\widehat{\mathbb{Z}}^{\times}$indeterminacies, one can only recover the $\widehat{\mathbb{Z}}^{\times}$-orbit of the MLF-Galois-pair-theoretic cyclotomic rigidity isomorphism.

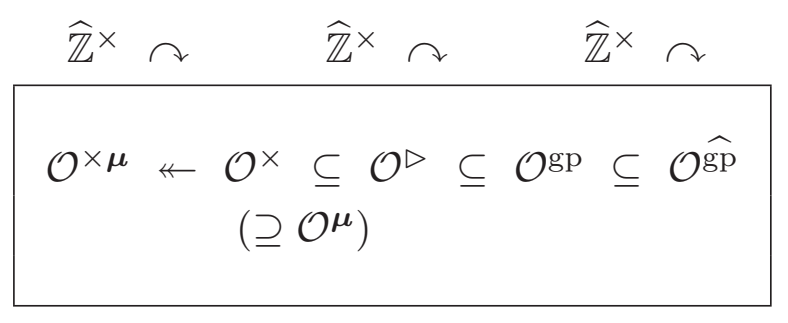

Fig. 2.1: Induced $\widehat{\mathbb{Z}}^{\times}$-indeterminacies in the case of MLF-Galois pair cyclotomic rigidity

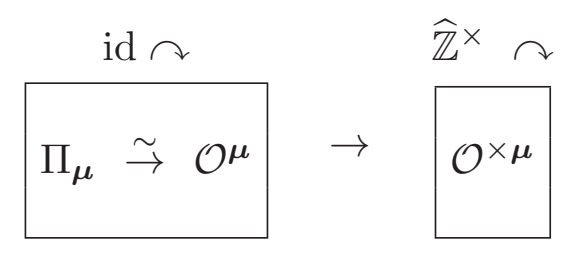

Fig. 2.2: Insulation from $\widehat{\mathbb{Z}}^{\times}$-indeterminacies in the case of mono-theta-theoretic cyclotomic rigidity

(iv) Thus, in summary, [cf. Fig. 2.2 above]

mono-theta-theoretic cyclotomic rigidity plays an essential role in the theory of the present $\S 2$ - and, indeed, in the theory of the present series of papers! - in that it serves to insulate the étale theta function from the $\widehat{\mathbb{Z}}^{\times}$-indeterminacies which act on the coric log-shells [i.e., the various monoids " $(-)^{\times \mu}$ "].

The techniques that underlie the resulting multiradiality of theta monoids [cf. Corollary 2.3 below], cannot, however, be applied immediately to the case of Gaussian 
monoids. That is to say, the corresponding multiradiality of Gaussian monoids, to be discussed in $\S 3$ below, requires one to apply the theory of log-shells developed in $\S 1$ [cf. [IUTchII], Remark 2.9.1, (iii); [IUTchII], Remark 3.4.1, (ii); [IUTchII], Remark 3.7.1]. On the other hand, as we shall see in $\S 3$ below, the multiradiality of Gaussian monoids depends in an essential way on the multiradiality of theta monoids discussed in the present $\S 2$ as a sort of "essential first step" constituted by the decoupling discussed in (ii) above. Indeed, if one tries to consider the Kummer theory of the theta values [i.e., the " $\underline{\underline{\underline{q}}} \underline{\underline{\underline{v}}} \underline{\underline{\underline{v}}}$ " - cf. [IUTchII], Remark 2.5.1, (i)] just as elements of the base field - i.e., without availing oneself of the theory of the étale theta function - then it is difficult to see how to rigidify the cyclotomes involved by any means other than the theory of $M L F$-Galois pairs discussed in (iii) above. But, as discussed in (iii) above, this approach to cyclotomic rigidity gives rise to $\widehat{\mathbb{Z}}^{\times}$-indeterminacies - i.e., to confusion between the theta values " $\underline{\underline{q}} \underline{\underline{\underline{j}}} \underline{\underline{v}}$ " and their $\widehat{\mathbb{Z}}^{\times}$-powers, which is unacceptable from the point of view of the theory of the present series of papers! For another approach to understanding the indispensability of the multiradiality of theta monoids, we refer to Remark 2.2.2 below.

\section{Remark 2.2.2.}

(i) One way to understand the very special role played by the theta values [i.e., the values of the theta function] in the theory of the present series of papers is to consider the following naive question:

Can one develop a similar theory to the theory of the present series of papers in which one replaces the $\Theta_{\mathrm{gau}}^{\times \mu}$-link

$$
\underline{\underline{q}} \mapsto \underline{\underline{q}}\left(\begin{array}{c}
1^{2} \\
\vdots \\
\left(l^{*}\right)^{2}
\end{array}\right)
$$

[cf. [IUTchII], Remark 4.11.1] by a correspondence of the form

$$
\underline{\underline{q}} \mapsto \underline{\underline{q}}^{\lambda}
$$

— where $\lambda$ is some arbitrary positive integer?

The answer to this question is "no". Indeed, such a correspondence does not come equipped with the extensive multiradiality machinery - such as monotheta-theoretic cyclotomic rigidity and the splittings determined by zerolabeled evaluation points - that has been developed for the the étale theta function. For instance, the lack of mono-theta-theoretic cyclotomic rigidity means that one does not have an apparatus for insulating the Kummer classes of such a correspondence from the $\widehat{\mathbb{Z}}^{\times}$-indeterminacies that act on the various monoids "(- $)^{\times \boldsymbol{\mu} "}[$ cf. the discussion of Remark 2.2.1, (iv)]. The splittings determined by zero-labeled evaluation points also play an essential role in decoupling these monoids " $(-)^{\times \mu}$ " — i.e., the coric log-shells — from the "purely radial" [or, 
put another way, "value group"] portion of such a correspondence "q $\underline{\underline{q}} \underline{\underline{q}}^{\lambda "}[\mathrm{cf}$. Remark 2.2.1, (ii); [IUTchII], Remark 1.12.2, (vi)]. Note, moreover, that if one tries to realize such a multiradial splitting via evaluation - i.e., in accordance with the principle of "Galois evaluation" [cf. the discussion of [IUTchII], Remark 1.12.4] - for a correspondence " $\underline{\underline{q}} \mapsto \underline{q}^{\lambda}$ " by, for instance, taking $\lambda$ to be one of the " $j^{2}$ " [where $j$ is a positive integer] that appears as a value of the étale theta function, then one must contend with issues of symmetry between the zero-labeled evaluation point and the evaluation point corresponding to $\lambda$ - i.e., symmetry issues that are resolved in the theory of the present series of papers by means of the theory surrounding the $\mathbb{F}_{l}^{\rtimes \pm}$-symmetry [cf. the discussion of [IUTchII], Remarks 2.6.2, 3.5.2]. As discussed in [IUTchII], Remark 2.6.3, this sort of situation leads to numerous conditions on the collection of evaluation points under consideration. In particular, ultimately, it is difficult to see how to construct a theory as in the present series of papers for any collection of evaluation points other than the collection that is in fact adopted in the definition of the $\Theta_{\mathrm{gau}}^{\times \boldsymbol{\mu}}$-link.

(ii) As discussed in Remark 2.2.1, (iv), we shall be concerned, in $\S 3$ below, with developing multiradial formulations for Gaussian monoids. These multiradial formulations will be subject to certain indeterminacies, which - although sufficiently mild to allow the execution of the volume computations that will be the subject of [IUTchIV] - are, nevertheless, substantially more severe than the indeterminacies that occur in the multiradial formulation given for theta monoids in the present $\S 2$ [cf. Corollary 2.3 below]. Indeed, the indeterminacies in the multiradial formulation given for theta monoids in the present $\S 2$ - which essentially consist of multiplication by roots of unity [cf. [IUTchII], Proposition 3.1, (i)] — are essentially negligible and may be regarded as a consequence of the highly nontrivial Kummer theory surrounding mono-theta environments [cf. Proposition 2.1, (iii); Theorem 2.2, (ii)], which, as discussed in Remark 2.2.1, (iv), cannot be mimicked for "theta values regarded just as elements of the base field". That is to say, the quite exact nature of the multiradial formulation for theta monoids - i.e., which contrasts sharply with the somewhat approximate nature of the multiradial formulation for Gaussian monoids to be developed in $\S 3$ - constitutes another important ingredient of the theory of the present paper that one must sacrifice if one attempts to work with correspondences $\underline{q} \mapsto \underline{q}^{\lambda}$ as discussed in (i), i.e., correspondences which do not come equipped with the extensive multiradiality machinery that arises as a consequence of the theory of the étale theta function developed in [EtTh].

We conclude the present $\S 3$ with the following multiradial interpretation [cf. [IUTchII], Remark 4.1.1, (iii); [IUTchII], Remark 4.3.1] — in the spirit of the étalepicture of $\mathcal{D}-\Theta^{ \pm \text {ell }} N F$-Hodge theaters of [IUTchII], Corollary 4.11, (ii) - of the theory surrounding Theorem 2.2.

Corollary 2.3. (Étale-picture of Multiradial Theta Monoids) In the notation of Theorem 2.2, let

$$
\left\{{ }^{n, m} \mathcal{H} \mathcal{T}^{\Theta^{ \pm e l l} \mathrm{NF}}\right\}_{n, m \in \mathbb{Z}}
$$

be a collection of distinct $\Theta^{ \pm e l l} \mathbf{N F}$-Hodge theaters [relative to the given initial $\Theta$-data] - which we think of as arising from a Gaussian log-theta-lattice [cf. 
Definition 1.4]. Write ${ }^{n, m} \mathcal{H} \mathcal{T}^{\mathcal{D}-\Theta^{ \pm \mathrm{ell}} \mathrm{NF}}$ for the $\mathcal{D}-\Theta^{ \pm \text {ell }} N F$-Hodge theater associated to ${ }^{n, m} \mathcal{H} \mathcal{T}^{\Theta^{ \pm e l l} \mathrm{NF}}$. Consider the radial environment [cf. [IUTchII], Example 1.7, (ii)] defined as follows. We define a collection of radial data

${ }^{\dagger} \mathfrak{R}=\left({ }^{\dagger} \mathcal{H} \mathcal{T}^{\mathcal{D}-\Theta^{ \pm \text {ell }} \mathrm{NF}}, \mathfrak{F}_{\text {env }}^{\Vdash}\left({ }^{\dagger} \mathfrak{D}_{>}\right),{ }^{\dagger} \mathfrak{R}^{\mathrm{bad}}, \mathfrak{F}_{\triangle}^{\vdash \times \boldsymbol{\mu}}\left({ }^{\dagger} \mathfrak{D}_{\triangle}^{\vdash}\right), \mathfrak{F}_{\text {env }}^{\vdash \times \boldsymbol{\mu}}\left({ }^{\dagger} \mathfrak{D}_{>}\right) \stackrel{\sim}{\rightarrow} \mathfrak{F}_{\triangle}^{\vdash \times \boldsymbol{\mu}}\left({ }^{\dagger} \mathfrak{D}_{\triangle}^{\vdash}\right)\right)$

to consist of

( $\left.a_{\mathfrak{R}}\right) \quad a \mathcal{D}-\Theta^{ \pm \mathrm{ell}} N F-H o d g e$ theater ${ }^{\dagger} \mathcal{H} \mathcal{T}^{\mathcal{D}-\Theta^{ \pm \mathrm{ell}} \mathrm{NF}}$;

$\left(b_{\mathfrak{R}}\right)$ the $\mathcal{F}^{\Vdash}$-prime-strip $\mathfrak{F}_{\text {env }}^{\Vdash}\left({ }^{\dagger} \mathfrak{D}_{>}\right)$associated to ${ }^{\dagger} \mathcal{H} \mathcal{T}^{\mathcal{D}-\Theta^{ \pm \mathrm{ell}} \mathrm{NF}}$ [cf. Proposition 2.1, (ii)];

$\left(c_{\mathfrak{R}}\right)$ the data $\left(a_{\underline{v}}\right),\left(b_{\underline{v}}\right),\left(c_{\underline{v}}\right),\left(d_{\underline{v}}\right)$ of Theorem 2.2, (ii), for $\underline{v} \in \underline{\mathbb{V}}^{\text {bad }}$, which we denote by ${ }^{\dagger} \mathfrak{R}^{\mathrm{bad}}$;

(d $\left.d_{\mathfrak{R}}\right)$ the $\mathcal{F}^{\vdash \times \boldsymbol{\mu}}$-prime-strip $\mathfrak{F}_{\triangle}^{\vdash \times \boldsymbol{\mu}}\left({ }^{\dagger} \mathfrak{D}_{\triangle}^{\vdash}\right)$ associated to ${ }^{\dagger} \mathcal{H} \mathcal{T}^{\mathcal{D}-\Theta^{ \pm \mathrm{ell}} \mathrm{NF}}$ [cf. Theorem 1.5, (iii)];

(e $\left.e_{\mathfrak{R}}\right)$ the full poly-isomorphism of $\mathcal{F}^{\vdash \times \boldsymbol{\mu}}$-prime-strips $\mathfrak{F}_{\text {env }}^{\vdash \times \boldsymbol{\mu}}\left({ }^{\dagger} \mathfrak{D}_{>}\right) \stackrel{\sim}{\rightarrow} \mathfrak{F}_{\triangle}^{\vdash \times \boldsymbol{\mu}}\left({ }^{\dagger} \mathfrak{D}_{\triangle}^{\vdash}\right)$.

We define a morphism between two collections of radial data ${ }^{\dagger} \mathfrak{R} \rightarrow{ }^{\ddagger} \mathfrak{R}$ [where we apply the evident notational conventions with respect to "4" and "F"] to consist of data as follows:

(a Mor $_{\mathfrak{R}}$ ) an isomorphism of $\mathcal{D}-\Theta^{ \pm \text {ell }} N F$-Hodge theaters ${ }^{\dagger} \mathcal{H} \mathcal{T}^{\mathcal{D}-\Theta^{ \pm \mathrm{ell}} \mathrm{NF}} \stackrel{\sim}{\rightarrow} \ddagger \mathcal{H} \mathcal{T}^{\mathcal{D}-\Theta^{ \pm \mathrm{ell}} \mathrm{NF}}$;

$\left(b_{\text {Mor }_{\mathfrak{R}}}\right)$ the isomorphism of $\mathcal{F}^{\Vdash}$-prime-strips $\mathfrak{F}_{\text {env }}^{\Vdash}\left({ }^{\dagger} \mathfrak{D}_{>}\right) \stackrel{\sim}{\rightarrow} \mathfrak{F}_{\text {env }}^{\Vdash}\left({ }^{\ddagger} \mathfrak{D}_{>}\right)$induced by the isomorphism of $\left(a_{\mathrm{Mor}_{\mathfrak{R}}}\right)$;

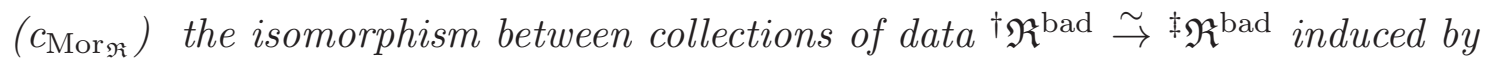
the isomorphism of $\left(a_{\mathrm{Mor}_{\mathfrak{R}}}\right)$;

$\left(d_{\operatorname{Mor}_{\mathfrak{R}}}\right)$ an isomorphism of $\mathcal{F}^{\vdash \times \boldsymbol{\mu}}$-prime-strips $\mathfrak{F}_{\triangle}^{\vdash \times \boldsymbol{\mu}}\left({ }^{\dagger} \mathfrak{D}_{\triangle}^{\vdash}\right) \stackrel{\sim}{\rightarrow} \mathfrak{F}_{\triangle}^{\vdash \times \boldsymbol{\mu}}\left({ }^{\ddagger} \mathfrak{D}_{\triangle}^{\vdash}\right)$;

$\left(e_{\text {Mor }_{\mathfrak{R}}}\right)$ we observe that the isomorphisms of $\left(b_{\text {Mor }_{\mathfrak{R}}}\right)$ and $\left(d_{\text {Mor }_{\mathfrak{R}}}\right)$ are necessarily compatible with the poly-isomorphisms of $\left(e_{\mathfrak{R}}\right)$ for "†", "f".

We define a collection of coric data

$$
{ }^{\dagger} \mathfrak{C}=\left({ }^{\dagger} \mathfrak{D}^{\vdash}, \mathfrak{F}_{\triangle}^{\vdash} \times \boldsymbol{\mu}\left({ }^{\dagger} \mathfrak{D}^{\vdash}\right)\right)
$$

to consist of

(ac $) \quad a \mathcal{D}^{\vdash}$-prime-strip ${ }^{\dagger} \mathfrak{D}^{\vdash}$;

(b $\left.b_{\mathfrak{C}}\right)$ the $\mathcal{F}^{\vdash \times \boldsymbol{\mu}}$-prime-strip $\mathfrak{F}_{\triangle}^{\vdash \times \boldsymbol{\mu}}\left({ }^{\dagger} \mathfrak{D}^{\vdash}\right)$ associated to ${ }^{\dagger} \mathfrak{D}^{\vdash}$ [cf. Theorem 1.5, (iii)]. 
We define a morphism between two collections of coric data ${ }^{\dagger} \mathfrak{C} \rightarrow \ddagger \mathfrak{C}$ [where we apply the evident notational conventions with respect to "4" and "F"] to consist of data as follows:

(a $a_{\text {Mor } \mathfrak{C}}$ ) an isomorphism of $\mathcal{D}^{\vdash}$-prime-strips ${ }^{\dagger} \mathfrak{D}^{\vdash} \stackrel{\sim}{\rightarrow} \ddagger \mathfrak{D}^{\vdash}$;

(b Mor $\left._{\mathfrak{C}}\right)$ an isomorphism of $\mathcal{F}^{\vdash \times \boldsymbol{\mu}}$-prime-strips $\mathfrak{F}_{\triangle}^{\vdash \times \boldsymbol{\mu}}\left({ }^{\dagger} \mathfrak{D}^{\vdash}\right) \stackrel{\sim}{\rightarrow} \mathfrak{F}_{\triangle}^{\vdash \times \boldsymbol{\mu}}\left({ }^{\ddagger} \mathfrak{D}^{\vdash}\right)$ that induces the isomorphism ${ }^{\dagger} \mathfrak{D}^{\vdash} \stackrel{\sim}{\rightarrow} \ddagger \mathfrak{D}^{\vdash}$ on associated $\mathcal{D}^{\vdash}$-prime-strips of $\left(a_{\text {Mor }_{\mathfrak{C}}}\right)$.

The radial algorithm is given by the assignment

$$
\begin{gathered}
{ }^{\dagger} \mathfrak{R}=\left({ }^{\dagger} \mathcal{H} \mathcal{T}^{\mathcal{D}-\Theta^{ \pm \text {ell }} \mathrm{NF}}, \mathfrak{F}_{\text {env }}^{\vdash}\left({ }^{\dagger} \mathfrak{D}_{>}\right),{ }^{\dagger} \mathfrak{R}^{\mathrm{bad}}, \mathfrak{F}_{\triangle}^{\vdash} \times \boldsymbol{\mu}\left({ }^{\dagger} \mathfrak{D}_{\triangle}^{\vdash}\right), \mathfrak{F}_{\mathrm{env}}^{\vdash \times \mu}\left({ }^{\dagger} \mathfrak{D}_{>}\right) \stackrel{\sim}{\rightarrow} \mathfrak{F}_{\triangle}^{\vdash \times \mu}\left({ }^{\dagger} \mathfrak{D}_{\triangle}^{\vdash}\right)\right) \\
\mapsto{ }^{\dagger} \mathfrak{C}=\left({ }^{\dagger} \mathfrak{D}_{\triangle}^{\vdash}, \mathfrak{F}_{\triangle}^{\vdash} \times \boldsymbol{\mu}\left({ }^{\dagger} \mathfrak{D}_{\triangle}^{\vdash}\right)\right)
\end{gathered}
$$

- together with the assignment on morphisms determined by the data of ( $\left.d_{\mathrm{Mor}_{\mathfrak{R}}}\right)$. Then:

(i) The functor associated to the radial algorithm defined above is full and essentially surjective. In particular, the radial environment defined above is multiradial.

(ii) Each $\mathcal{D}-\Theta^{ \pm \mathrm{ell}} N F$-Hodge theater ${ }^{n, m} \mathcal{H} \mathcal{T}^{\mathcal{D}-\Theta^{ \pm \mathrm{ell}} \mathrm{NF}}$, for $n, m \in \mathbb{Z}$, defines, in an evident way, an associated collection of radial data ${ }^{n, m} \mathfrak{R}$. The poly-isomorphisms induced by the vertical arrows of the Gaussian log-theta-lattice under consideration [cf. Theorem 1.5, (i)] induce poly-isomorphisms of radial data ... $\stackrel{\sim}{\rightarrow}{ }^{n, m} \mathfrak{R}$ $\stackrel{\sim}{\rightarrow} n, m+1 \mathfrak{\Re} \stackrel{\sim}{\rightarrow} \ldots$ Write

$$
{ }^{n, \circ} \mathfrak{R}
$$

for the collection of radial data obtained by identifying the various ${ }^{n, m} \mathfrak{R}$, for $m \in \mathbb{Z}$, via these poly-isomorphisms and ${ }^{n,}{ }^{\mathfrak{C}}$ for the collection of coric data associated, via the radial algorithm defined above, to the radial data ${ }^{n, \circ} \mathfrak{R}$. In a similar vein, the horizontal arrows of the Gaussian log-theta-lattice under consideration induce full poly-isomorphisms ... $\stackrel{\sim}{\rightarrow} n, m \mathfrak{D}_{\triangle}^{\vdash} \stackrel{\sim}{\rightarrow} n+1, m \mathfrak{D}_{\triangle}^{\vdash} \stackrel{\sim}{\rightarrow}$. . of $\mathcal{D}^{\vdash}$-prime-strips [cf. Theorem 1.5, (ii)]. Write

$$
{ }^{\circ, \circ} \mathfrak{C}
$$

for the collection of coric data obtained by identifying the various ${ }^{n, \circ} \mathfrak{C}$, for $n \in \mathbb{Z}$, via these poly-isomorphisms. Thus, by applying the radial algorithm defined above to each ${ }^{n, \circ} \mathfrak{R}$, for $n \in \mathbb{Z}$, we obtain a diagram - i.e., an étale-picture of radial data - as in Fig. 2.3 below. This diagram satisfies the important property of admitting arbitrary permutation symmetries among the spokes [i.e., the labels $n \in \mathbb{Z}$ ] and is compatible, in the evident sense, with the étale-picture of $\mathcal{D}-\Theta^{ \pm \mathrm{ell}} N F$-Hodge theaters of [IUTChII], Corollary 4.11, (ii).

(iii) The [poly-]isomorphisms of $\mathcal{F}^{\vdash \times \boldsymbol{\mu}}$-prime-strips of/induced by $\left(e_{\mathfrak{R}}\right),\left(b_{\mathrm{Mor}_{\mathfrak{R}}}\right)$, $\left(d_{\operatorname{Mor}_{\mathfrak{R}}}\right)$ [cf. also ( $\left.\left.e_{\operatorname{Mor}_{\mathfrak{R}}}\right)\right]$ are compatible, relative to the Kummer isomorphisms of Proposition 2.1, (ii) [cf. also Proposition 2.1, (vi)], and Theorem 1.5, (iii), with the poly-isomorphisms - arising from the horizontal arrows of the Gaussian log-theta-lattice - of Theorem 1.5, (ii). 


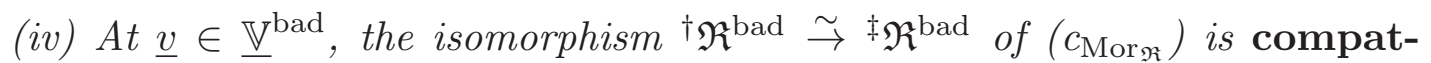
ible [cf. the final portion of Theorem 2.2, (ii)], relative to the Kummer isomorphisms and poly-isomorphisms of projective systems of mono-theta environments discussed in Proposition 2.1, (ii), (iii) [cf. also Proposition 2.1, (vi); the second display of Theorem 2.2, (ii)], and Theorem 1.5, (iii), with the poly-isomorphisms - arising from the horizontal arrows of the Gaussian logtheta-lattice - of Theorem 1.5, (ii).

Proof. The various assertions of Corollary 2.3 follow immediately from the definitions and the references quoted in the statements of these assertions.

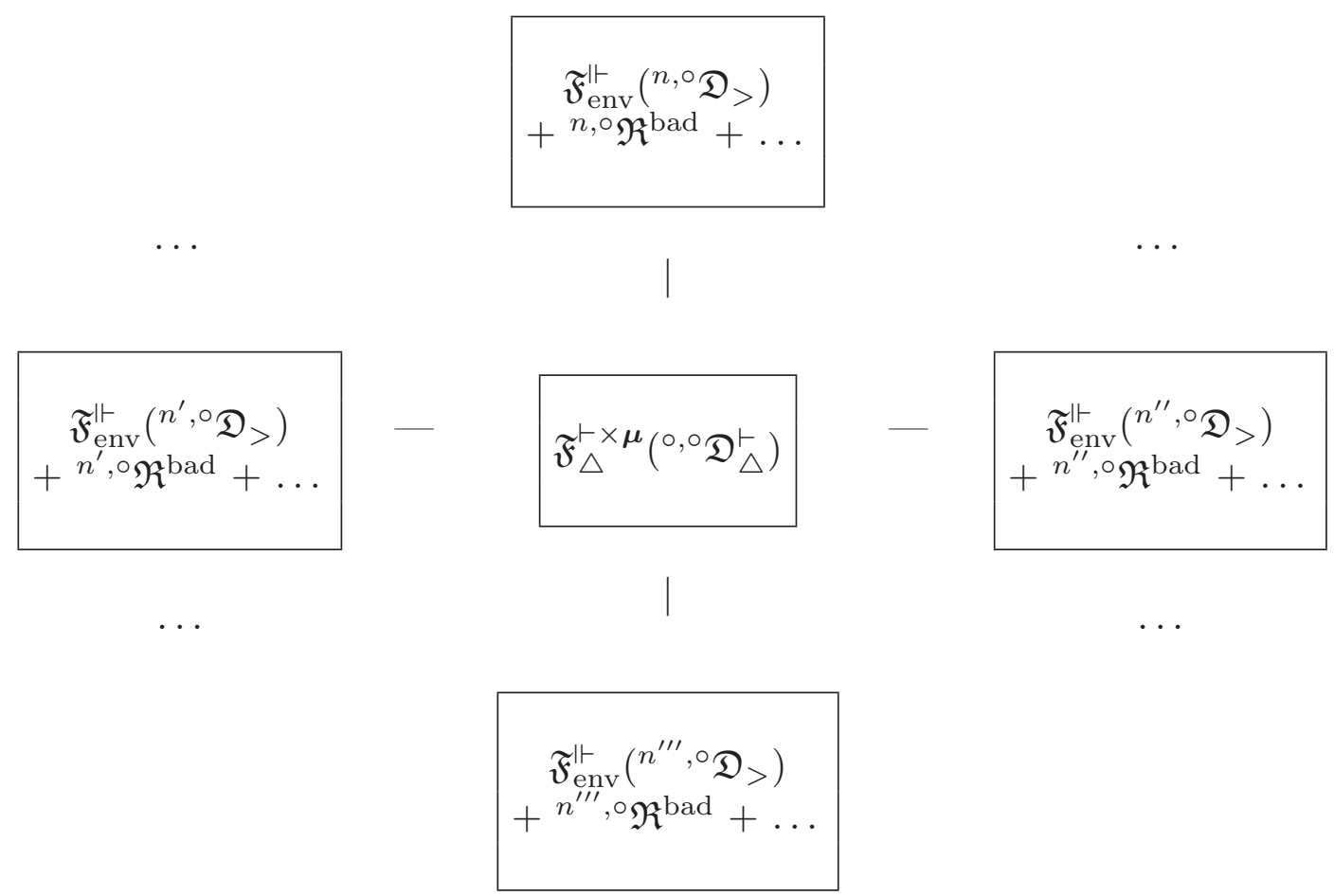

Fig. 2.3: Étale-picture of radial data

\section{Remark 2.3.1.}

(i) In the context of the étale-picture of Fig. 2.3, it is of interest to recall the point of view of the discussion of [IUTchII], 1.12.5, (i), (ii), concerning the analogy between étale-pictures in the theory of the present series of papers and the polar coordinate representation of the classical Gaussian integral.

(ii) The étale-picture discussed in Corollary 2.3, (ii), may be thought of as a sort of canonical splitting of the portion of the Gaussian log-theta-lattice under consideration that involves theta monoids [cf. the discussion of [IUTchI], $\S \mathrm{I1}$, preceding Theorem A].

(iii) The portion of the multiradiality discussed in Corollary 2.3, (iv), at $\underline{v} \in \underline{\mathbb{V}}^{\text {bad }}$ corresponds, in essence, to the multiradiality discussed in [IUTchII], Corollary 1.12, (iii); [IUTchII], Proposition 3.4, (i). 


\section{Definition 2.4.}

(i) Let

$$
{ }^{\ddagger} \mathfrak{F}^{\vdash}=\left\{{ }^{\ddagger} \mathcal{F}_{\underline{v}}^{\vdash}\right\}_{\underline{v} \in \underline{\mathbb{V}}}
$$

be an $\mathcal{F}^{\vdash}$-prime-strip. Then recall from the discussion of [IUTchII], Definition 4.9, (ii), that at each $\underline{w} \in \underline{\mathbb{V}}^{\text {bad }}$, the splittings of the split Frobenioid $\ddagger \mathcal{F}_{\underline{w}}^{\vdash}$ determine submonoids " $\mathcal{O}^{\perp}(-) \subseteq \mathcal{O}^{\triangleright}(-)$ ", as well as quotient monoids " $\mathcal{O}^{\perp}(-) \rightarrow \mathcal{O}^{\triangleright}(-)$ " [i.e., by forming the quotient of " $\mathrm{O}^{\perp}(-)$ " by its torsion subgroup]. In a similar vein, for each $\underline{w} \in \underline{\mathbb{V}}^{\text {good }}$, the splitting of the split Frobenioid determined by [indeed, "constituted by", when $\underline{w} \in \underline{\mathbb{V}}^{\text {good }} \cap \underline{\mathbb{V}}^{\text {non }}$ - cf. [IUTchI], Definition 5.2, (ii)] ${ }^{\ddagger} \mathcal{F}_{\underline{w}}^{\vdash}$ determines a submonoid " $\mathcal{O}^{\perp}(-) \subseteq \mathcal{O}^{\triangleright}(-)$ " whose subgroup of units is trivial [cf. [IUTchII], Definition 4.9, (iv), when $\left.\underline{w} \in \underline{\mathbb{V}}^{\text {good }} \cap \underline{\mathbb{V}}^{\text {non }}\right]$; in this case, we set $\mathcal{O} \triangleright(-) \stackrel{\text { def }}{=} \mathcal{O}^{\perp}(-)$. Write

$$
{ }^{\ddagger} \mathfrak{F}^{\vdash \perp}=\left\{{ }^{\ddagger} \mathcal{F}_{\underline{\underline{v}}}^{\vdash \perp}\right\}_{\underline{v} \in \underline{\mathbb{V}}} ; \quad{ }^{\ddagger} \mathfrak{F}^{\vdash}=\left\{{ }^{\ddagger} \mathcal{F}_{\underline{v}}^{\vdash}\right\}_{\underline{v} \in \underline{\mathbb{V}}}
$$

for the collections of data obtained by replacing the split Frobenioid portion of each ${ }^{\ddagger} \mathcal{F}_{\underline{v}}^{\vdash}$ by the Frobenioids determined, respectively, by the subquotient monoids " $\mathcal{O}^{\perp}(-) \subseteq \mathcal{O}^{\triangleright}(-)$ ", " $\mathcal{O} \triangleright(-)$ " just defined.

(ii) We define [in the spirit of [IUTchII], Definition 4.9, (vii)] an $\mathcal{F}^{\vdash \perp}$-primestrip to be a collection of data

$$
* \mathfrak{F}^{\vdash \perp}=\left\{{ }^{*} \mathcal{F}_{\underline{v}}^{\vdash \perp}\right\}_{\underline{v} \in \underline{\mathbb{V}}}
$$

that satisfies the following conditions: (a) if $\underline{v} \in \underline{\mathbb{V}}^{\text {non }}$, then ${ }^{*} \mathcal{F}_{\underline{v}}^{+\perp}$ is a Frobenioid that is isomorphic to ${ }^{\ddagger} \mathcal{F}_{\underline{v}}^{\vdash} \perp$ cf. (i)]; (b) if $\underline{v} \in \underline{\mathbb{V}}^{\text {arc }}$, then ${ }^{*} \mathcal{F}_{\underline{v}}^{\vdash}$ consists of a Frobenioid and an object of $\mathbb{T M}^{\vdash}$ [cf. [IUTchI], Definition 5.2, (ii)] such that ${ }^{*} \mathcal{F}_{\underline{v}}^{\vdash \perp}$ is isomorphic to $\ddagger \mathcal{F}_{\underline{v}}^{\vdash \perp}$. In a similar vein, we define an $\mathcal{F}^{\vdash}$-prime-strip to be a collection of data

$$
* \mathfrak{F} \vdash=\left\{{ }^{*} \mathcal{F}_{\underline{v}}^{\vdash}\right\}_{\underline{v} \in \underline{\mathbb{V}}}
$$

that satisfies the following conditions: (a) if $\underline{v} \in \underline{\mathbb{V}}^{\text {non }}$, then ${ }^{*} \mathcal{F}_{\underline{v}}^{\vdash}$ is a Frobenioid that is isomorphic to ${ }^{\ddagger} \mathcal{F}_{\underline{v}}^{\vdash}\left[\mathrm{cf}\right.$. (i)]; (b) if $\underline{v} \in \underline{\mathbb{V}}^{\text {arc }}$, then ${ }^{*} \mathcal{F}_{\underline{v}}^{\vdash}$ consists of a Frobenioid and an object of $\mathbb{T M}^{\vdash}$ [cf. [IUTchI], Definition 5.2, (ii)] such that ${ }^{*} \mathcal{F}_{\underline{v}}^{+}$ is isomorphic to ${ }^{\ddagger} \mathcal{F}_{\underline{v}}^{\vdash}$. A morphism of $\mathcal{F}^{\vdash \perp}$ - (respectively, $\mathcal{F}^{\vdash} \bullet_{-}$) prime-strips is defined to be a collection of isomorphisms, indexed by $\underline{\mathbb{V}}$, between the various constituent objects of the prime-strips [cf. [IUTchI], Definition 5.2, (iii)].

(iii) We define [in the spirit of [IUTchII], Definition 4.9, (viii)] an $\mathcal{F}^{\Vdash \perp}$-primestrip to be a collection of data

$$
{ }^{*} \mathfrak{F}^{\Vdash \perp}=\left({ }^{*} \mathcal{C}^{\Vdash}, \operatorname{Prime}\left({ }^{*} \mathcal{C}^{\Vdash}\right) \stackrel{\sim}{\rightarrow} \underline{\mathbb{V}},{ }^{*} \mathfrak{F}^{\vdash \perp},\left\{{ }^{*} \rho_{\underline{v}}\right\}_{\underline{v} \in \underline{\mathbb{V}}}\right)
$$

satisfying the conditions (a), (b), (c), (d), (e), (f) of [IUTchI], Definition 5.2, (iv), for an $\mathcal{F}^{\Vdash}$-prime-strip, except that the portion of the collection of data constituted by an $\mathcal{F}^{\vdash}$-prime-strip is replaced by an $\mathcal{F}^{\vdash}+$-prime-strip. [We leave the routine 
details to the reader.] In a similar vein, we define an $\mathcal{F}^{\Vdash}$-prime-strip to be a collection of data

$$
{ }^{*} \mathfrak{F}^{\Vdash \triangleright}=\left({ }^{*} \mathcal{C}^{\Vdash}, \operatorname{Prime}\left({ }^{*} \mathcal{C}^{\Vdash}\right) \stackrel{\sim}{\rightarrow} \underline{\mathbb{V}},{ }^{*} \mathfrak{F}^{\vdash},\left\{{ }^{*} \rho_{\underline{v}}\right\}_{\underline{v} \in \underline{\mathbb{V}}}\right)
$$

satisfying the conditions (a), (b), (c), (d), (e), (f) of [IUTchI], Definition 5.2, (iv), for an $\mathcal{F}^{\Vdash}$-prime-strip, except that the portion of the collection of data constituted by an $\mathcal{F}^{\vdash}$-prime-strip is replaced by an $\mathcal{F}^{\vdash}-$-prime-strip. [We leave the routine details to the reader.] A morphism of $\mathcal{F}^{\Vdash \perp} \perp_{-}$(respectively, $\mathcal{F}^{\Vdash} \boldsymbol{-}_{-}$) prime-strips is defined to be an isomorphism between collections of data as discussed above.

\section{Remark 2.4.1.}

(i) Thus, by applying the constructions of Definition 2.4, (i), to the [underlying $\mathcal{F}^{\vdash}$-prime-strips associated to the] $\mathcal{F}^{\Vdash}$-prime-strips " $\mathfrak{F}_{\text {env }}^{\vdash}\left({ }^{\dagger} \mathfrak{D}_{>}\right)$" that appear in Corollary 2.3, one may regard the multiradiality of Corollary 2.3, (i), as implying a corresponding multiradiality assertion concerning the associated $\mathcal{F}^{\Vdash \perp}$-primestrips “ $\mathfrak{F}_{\text {env }}^{\vdash \perp}\left(\dagger \mathfrak{D}_{>}\right)$".

(ii) Suppose that we are in the situation discussed in (i). Then at $\underline{v} \in \underline{\mathbb{V}}^{\text {bad }}$, the submonoids " $\mathcal{O}^{\perp}(-) \subseteq \mathcal{O}^{\triangleright}(-)$ " may be regarded, in a natural way [cf. Proposition 2.1 , (ii); Theorem 2.2 , (ii)], as submonoids of the monoids " $\infty \Psi_{\mathrm{env}}^{\perp}\left({ }^{\dagger} \mathfrak{D}_{>}\right)_{\underline{\underline{v}}}$ " of Theorem 2.2, (ii), $\left(\mathrm{a}_{\underline{v}}\right)$. Moreover, the resulting inclusion of monoids is compatible with the multiradiality discussed in (i) and the multiradiality of the data "† $\mathfrak{R}^{\text {bad" }}$ " of Corollary 2.3, $\left(\mathrm{c}_{\mathfrak{R}}\right)$, that is implied by the multiradiality of Corollary 2.3, (i). 


\section{Section 3: Multiradial Logarithmic Gaussian Procession Monoids}

In the present $\S 3$, we apply the theory developed thus far in the present series of papers to give [cf. Theorem 3.11 below] multiradial algorithms for a slightly modified version of the Gaussian monoids discussed in [IUTchII], §4. This modification revolves around the combinatorics of processions, as developed in [IUTchI], $\S 4, \S 5, \S 6$, and is necessary in order to establish the desired multiradiality. At a more concrete level, these combinatorics require one to apply the theory of tensor packets [cf. Propositions 3.1, 3.2, 3.3, 3.4, 3.7, 3.9, below]. Finally, we observe in Corollary 3.12 that these multiradial algorithms give rise to certain estimates concerning the log-volumes of the logarithmic Gaussian procession monoids that occur. This observation forms the starting point of the theory to be developed in [IUTchIV].

In the following discussion, we assume that we have been given initial $\Theta$-data as in [IUTchI], Definition 3.1. Also, we shall write

$$
\mathbb{V}_{\mathbb{Q}} \stackrel{\text { def }}{=} \mathbb{V}(\mathbb{Q})
$$

[cf. [IUTchI], §0] and apply the notation of Definition 1.1 of the present paper. We begin by discussing the theory of tensor packets, which may be thought of as a sort of amalgamation of the theory of log-shells developed in $\$ 1$ with the theory of processions developed in [IUTchI], $44, \S 5, \S 6$.

\section{Proposition 3.1. $\quad$ (Local Holomorphic Tensor Packets) Let}

$$
\left\{{ }^{\alpha} \mathfrak{F}\right\}_{\alpha \in A}=\left\{\left\{{ }^{\alpha} \mathcal{F}_{\underline{v}}\right\}_{\underline{v} \in \mathbb{V}}\right\}_{\alpha \in A}
$$

be an $\boldsymbol{n}$-capsule, with index set $A$, of $\mathcal{F}$-prime-strips [relative to the given initial $\Theta$-data - cf. [IUTchI], §0; [IUTchI], Definition 5.2, (i)]. Then [cf. the notation of Definition 1.1, (iii)] we shall refer to the correspondence

$$
\mathbb{V}_{\mathbb{Q}} \ni v_{\mathbb{Q}} \mapsto \underline{\mathfrak{l o g}}\left({ }^{\alpha} \mathcal{F}_{v_{\mathbb{Q}}}\right) \stackrel{\text { def }}{=} \bigoplus_{\underline{\mathbb{V}} \ni \underline{v} \mid v_{\mathbb{Q}}} \underline{\mathfrak{l o g}}\left({ }^{\alpha} \mathcal{F}_{\underline{v}}\right)
$$

as the [1-]tensor packet associated to the $\mathcal{F}$-prime-strip ${ }^{\alpha} \mathfrak{F}$ and to the correspondence

$$
\mathbb{V}_{\mathbb{Q}} \ni v_{\mathbb{Q}} \mapsto \underline{\log }\left({ }^{A} \mathcal{F}_{v_{\mathbb{Q}}}\right) \stackrel{\text { def }}{=} \bigotimes_{\alpha \in A} \underline{\log }\left({ }^{\alpha} \mathcal{F}_{v_{\mathbb{Q}}}\right)
$$

- where the tensor product is to be understood as a tensor product of topological modules - as the $[\boldsymbol{n}$-] tensor packet associated to the collection of $\mathcal{F}$-prime-strips $\left\{{ }^{\alpha} \mathfrak{F}\right\}_{\alpha \in A}$. Then:

(i) (Ring Structures) The topological field structures on the various $\underline{\log }\left({ }^{\alpha} \mathcal{F}_{\underline{v}}\right)$ [cf. Definition 1.1, (i), (ii), (iii)], for $\alpha \in A$, determine a topolog-

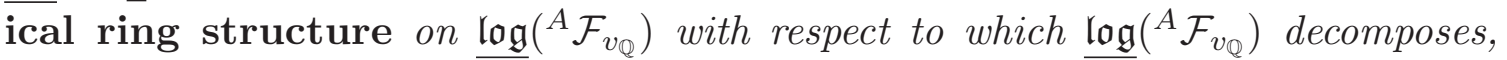
uniquely, as a direct sum of topological fields, in a fashion that is compatible, 
for $\alpha \in A$, with the natural action of the topological group ${ }^{\alpha} \Pi_{\underline{v}}$ [where $\underline{\mathbb{V}} \ni \underline{v} \mid v_{\mathbb{Q}}$ ] on the direct summand with subscript $\underline{v}$ of the factor labeled $\alpha$.

(ii) (Integral Structures) Fix elements $\alpha \in A, \underline{v} \in \underline{\mathbb{V}}, v_{\mathbb{Q}} \in \mathbb{V}_{\mathbb{Q}}$ such that $\underline{v} \mid v_{\mathbb{Q}}$. Relative to the tensor product in the above definition of $\underline{\log }\left({ }^{A} \mathcal{F}_{v_{\mathbb{Q}}}\right)$, write

$$
\underline{\mathfrak{l o g}}\left({ }^{A, \alpha} \mathcal{F}_{\underline{v}}\right) \stackrel{\text { def }}{=} \underline{\mathfrak{l o g}}\left({ }^{\alpha} \mathcal{F}_{\underline{v}}\right) \otimes\left\{\bigotimes_{\beta \in A \backslash\{\alpha\}} \underline{\mathfrak{l o g}}\left({ }^{\beta} \mathcal{F}_{v_{\mathbb{Q}}}\right)\right\} \subseteq \underline{\mathfrak{l o g}}\left({ }^{A} \mathcal{F}_{v_{\mathbb{Q}}}\right)
$$

for the topological submodule determined by the tensor product of the factors labeled by $\beta \in A \backslash\{\alpha\}$ with the tensor product of the direct summand with subscript $\underline{v}$ of the factor labeled $\alpha$. Then $\underline{\log }\left({ }^{A, \alpha} \mathcal{F}_{\underline{v}}\right)$ forms a direct summand of the topological ring $\log \left({ }^{A} \mathcal{F}_{v_{\mathbb{Q}}}\right)$; the topological ring structure on $\log \left({ }^{A, \alpha} \mathcal{F}_{\underline{v}}\right)$ decomposes, uniquely, as a direct sum of topological fields. Moreover, by forming the tensor product with " 1 's" in the factors labeled by $\beta \in A \backslash\{\alpha\}$, one obtains a natural injective homomorphism of topological rings

$$
\underline{\log }\left({ }^{\alpha} \mathcal{F}_{\underline{v}}\right) \rightarrow \underline{\log }\left({ }^{A, \alpha} \mathcal{F}_{\underline{v}}\right)
$$

that induces an isomorphism of the domain onto each of the direct summand topological fields of the codomain. In particular, the integral structure

$$
\bar{\Psi}_{\mathfrak{l o g}\left(\alpha \mathcal{F}_{\underline{v}}\right)} \stackrel{\text { def }}{=} \Psi_{\mathfrak{l o g}\left(\alpha \mathcal{F}_{\underline{v}}\right)} \bigcup\{0\} \subseteq \underline{\log }\left({ }^{\alpha} \mathcal{F}_{\underline{v}}\right)
$$

[cf. the notation of Definition 1.1, (i), (ii)] determines integral structures on each of the direct summand topological fields of $\underline{\mathfrak{l o g}}\left({ }^{A, \alpha} \mathcal{F}_{\underline{v}}\right), \underline{\mathfrak{l o g}}\left({ }^{A} \mathcal{F}_{v_{\mathbb{Q}}}\right)$.

Proof. The various assertions of Proposition 3.1 follow immediately from the definitions and the references quoted in the statements of these assertions [cf. also Remark 3.1.1, (i), below].

\section{Remark 3.1.1.}

(i) Let $\underline{v} \in \underline{\mathbb{V}}$. In the notation of [IUTchI], Definition 3.1 , write $k \stackrel{\text { def }}{=} K_{v}$; let $\bar{k}$ be an algebraic closure of $k$. Then, roughly speaking, in the notation of Proposition 3.1 ,

$$
\begin{aligned}
& \underline{\log }\left({ }^{\alpha} \mathcal{F}_{\underline{v}}\right) \quad \stackrel{\sim}{\rightarrow} \quad \bar{k} ; \quad \bar{\Psi}_{\mathfrak{l o g}\left(\alpha \mathcal{F}_{\underline{v}}\right)} \stackrel{\sim}{\rightarrow} \quad \mathcal{O}_{\bar{k}} ; \\
& \underline{\log }\left({ }^{A, \alpha} \mathcal{F}_{\underline{v}}\right) \stackrel{\sim}{\rightarrow} \bigotimes \bar{k} \stackrel{\sim}{\rightarrow} \bigoplus \bar{k} \supseteq \bigoplus \mathcal{O}_{\bar{k}}
\end{aligned}
$$

—i.e., one verifies immediately that each topological field $\underline{\log }\left({ }^{\alpha} \mathcal{F}_{\underline{v}}\right)$ is isomorphic to $\bar{k}$; each $\log \left({ }^{A, \alpha} \mathcal{F}_{\underline{v}}\right)$ is a tensor product [say, over $\mathbb{Q}$ ] of copies of $\bar{k}$, hence decomposes as a direct sum of copies of $\bar{k}$; each $\bar{\Psi}_{\mathfrak{l o g}\left({ }^{\alpha} \mathcal{F}_{\underline{v}}\right)}$ is a copy of the set [i.e., a ring, when $\left.\underline{v} \in \underline{\mathbb{V}}^{\text {non }}\right]$ of integers $\mathcal{O}_{\bar{k}} \subseteq \bar{k}$. In particular, the "integral structures" discussed in the final portion of Proposition 3.1, (ii), correspond to copies of $\mathcal{O}_{\bar{k}}$ contained in copies of $\bar{k}$.

(ii) Ultimately, for $\underline{v} \in \underline{\mathbb{V}}$, we shall be interested [cf. Proposition 3.9, (i), (ii), below] in considering log-volumes on the portion of $\underline{\log }\left({ }^{\alpha} \mathcal{F}_{\underline{v}}\right)$ corresponding to 
$K_{v}$. On the other hand, let us recall that we do not wish to consider all of the valuations in $\mathbb{V}(K)$. That is to say, we wish to restrict ourselves to considering the subset $\underline{\mathbb{V}} \subseteq \mathbb{V}(K)$, equipped with the natural bijection $\underline{\mathbb{V}} \stackrel{\sim}{\rightarrow} \mathbb{V}_{\bmod }$ [cf. [IUTchI], Definition 3.1, (e)], which we wish to think of as a sort of "local analytic section" [cf. the discussion of [IUTchI], Remark 4.3.1, (i)] of the natural morphism $\operatorname{Spec}(K) \rightarrow$ $\operatorname{Spec}(F)$ [or, perhaps more precisely, $\left.\operatorname{Spec}(K) \rightarrow \operatorname{Spec}\left(F_{\text {mod }}\right)\right]$. In particular, it will be necessary to consider these log-volumes on the portion of $\underline{l o g}\left({ }^{\alpha} \mathcal{F}_{\underline{v}}\right)$ corresponding to $K_{\underline{v}}$ relative to the weight $\left[K_{\underline{v}}:\left(F_{\bmod }\right)_{v}\right]^{-1}$, where we write $v \in \mathbb{V}_{\text {mod }}$ for the element determined [via the natural bijection just discussed] by $\underline{v}$ [cf. the discussion of [IUTchI], Example 3.5, (i), (ii), (iii), where similar factors appear]. When, moreover, we consider direct sums over all $\underline{v} \in \underline{\mathbb{V}}$ lying over a given $v_{\mathbb{Q}} \in \mathbb{V}_{\mathbb{Q}}$ as in the case of $\underline{\log }\left({ }^{\alpha} \mathcal{F}_{v_{\mathbb{Q}}}\right)$, it will be convenient to use the normalized weight

$$
\frac{1}{\left[K_{\underline{v}}:\left(F_{\bmod }\right)_{v}\right] \cdot\left(\sum_{\mathbb{V}_{\bmod } \ni w \mid v_{\mathbb{Q}}}\left[\left(F_{\bmod }\right)_{w}: \mathbb{Q}_{v_{\mathbb{Q}}}\right]\right)}
$$

— i.e., normalized so that multiplication by $p_{v_{\mathbb{Q}}}$ affects $\log$-volumes by addition or subtraction [that is to say, depending on whether $v_{\mathbb{Q}} \in \mathbb{V}_{\mathbb{Q}}^{\text {arc }}$ or $v_{\mathbb{Q}} \in \mathbb{V}_{\mathbb{Q}}^{\text {non }}$ ] of the quantity $\log \left(p_{v_{\mathbb{Q}}}\right) \in \mathbb{R}$. In a similar vein, when we consider $\log$-volumes on the portion of $\underline{\log }\left({ }^{A} \mathcal{F}_{v_{\mathbb{Q}}}\right)$ corresponding to the tensor product of various $K_{\underline{v}_{\alpha}}$, where $\underline{\mathbb{V}} \ni \underline{v}_{\alpha} \mid v_{\mathbb{Q}}$, it will be necessary to consider these log-volumes relative to the weight

$$
\frac{1}{\prod_{\alpha \in A}\left[K_{\underline{v}_{\alpha}}:\left(F_{\mathrm{mod}}\right) v_{\alpha}\right]}
$$

— where we write $v_{\alpha} \in \mathbb{V}_{\text {mod }}$ for the element determined by $\underline{v}_{\alpha}$. When, moreover, we consider direct sums over all possible choices for the data $\left\{\underline{v}_{\alpha}\right\}_{\alpha \in A}$, it will be convenient to use the normalized weight

$$
\frac{1}{\left(\prod_{\alpha \in A}\left[K_{\underline{v}_{\alpha}}:\left(F_{\bmod }\right)_{v_{\alpha}}\right]\right) \cdot\left\{\sum_{\left\{w_{\alpha}\right\}_{\alpha \in A}}\left(\prod_{\alpha \in A}\left[\left(F_{\bmod }\right)_{w_{\alpha}}: \mathbb{Q}_{v_{\mathbb{Q}}}\right]\right)\right\}}
$$

- where the sum is over all collections $\left\{w_{\alpha}\right\}_{\alpha \in A}$ of [not necessarily distinct!] elements $w_{\alpha} \in \mathbb{V}_{\text {mod }}$ lying over $v_{\mathbb{Q}}$ and indexed by $\alpha \in A$. Again, these normalized weights are normalized so that multiplication by $p_{v_{\mathbb{Q}}}$ affects log-volumes by addition or subtraction [that is to say, depending on whether $v_{\mathbb{Q}} \in \mathbb{V}_{\mathbb{Q}}^{\text {arc }}$ or $v_{\mathbb{Q}} \in \mathbb{V}_{\mathbb{Q}}^{\text {non }}$ ] of the quantity $\log \left(p_{v_{\mathbb{Q}}}\right) \in \mathbb{R}$.

Remark 3.1.2. The constructions involving local holomorphic tensor packets given in Proposition 3.1 may be applied to the capsules that appear in the various $\mathcal{F}$-prime-strip processions obtained by considering the evident $\mathcal{F}$-prime-strip analogues [cf. [IUTchI], Remark 5.6.1; [IUTchI], Remark 6.12.1] of the holomorphic processions discussed in [IUTchI], Proposition 4.11, (i); [IUTchI], Proposition $6.9,(\mathrm{i})$. 
Proposition 3.2. (Local Mono-analytic Tensor Packets) Let

$$
\left\{{ }^{\alpha} \mathfrak{D}^{\vdash}\right\}_{\alpha \in A}=\left\{\left\{^{\alpha} \mathcal{D}_{\underline{v}}^{\vdash}\right\}_{\underline{v} \in \underline{\mathbb{V}}}\right\}_{\alpha \in A}
$$

be an $\boldsymbol{n}$-capsule, with index set $A$, of $\mathcal{D}^{\vdash}$-prime-strips /relative to the given initial $\Theta$-data - cf. [IUTchI], §0; [IUTchI], Definition 4.1, (iii)]. Then [cf. the notation of Proposition 1.2, (vi), (vii)] we shall refer to the correspondence

$$
\mathbb{V}_{\mathbb{Q}} \ni v_{\mathbb{Q}} \mapsto \underline{\mathfrak{l o g}}\left({ }^{\alpha} \mathcal{D}_{v_{\mathbb{Q}}}^{\vdash}\right) \stackrel{\text { def }}{=} \bigoplus_{\underline{\mathbb{V}} \ni \underline{v} \mid v_{\mathbb{Q}}} \underline{\mathfrak{l o g}}\left({ }^{\alpha} \mathcal{D}_{\underline{v}}^{\vdash}\right)
$$

as the [1-]tensor packet associated to the $\mathcal{D}^{\vdash}$-prime-strip ${ }^{\alpha} \mathfrak{D}^{\vdash}$ and to the correspondence

$$
\mathbb{V}_{\mathbb{Q}} \ni v_{\mathbb{Q}} \mapsto \underline{\mathfrak{l o g}}\left({ }^{A} \mathcal{D}_{v_{\mathbb{Q}}}^{\vdash}\right) \stackrel{\text { def }}{=} \bigotimes_{\alpha \in A} \underline{\log }\left({ }^{\alpha} \mathcal{D}_{v_{\mathbb{Q}}}^{\vdash}\right)
$$

- where the tensor product is to be understood as a tensor product of topological modules - as the [n-] tensor packet associated to the collection of $\mathcal{D}^{\vdash}$-prime-strips $\left\{{ }^{\alpha} \mathfrak{D}^{\vdash}\right\}_{\alpha \in A}$. For $\alpha \in A, \underline{v} \in \underline{\mathbb{V}}, v_{\mathbb{Q}} \in \mathbb{V}_{\mathbb{Q}}$ such that $\underline{v} \mid v_{\mathbb{Q}}$, we shall write

$$
\underline{\mathfrak{l o g}}\left({ }^{A, \alpha} \mathcal{D}_{\underline{v}}^{\vdash}\right) \subseteq \underline{\log }\left({ }^{A} \mathcal{D}_{v_{\mathbb{Q}}}^{\vdash}\right)
$$

for the topological submodule determined by the tensor product of the factors labeled by $\beta \in A \backslash\{\alpha\}$ with the tensor product of the direct summand with subscript $\underline{v}$ of the factor labeled $\alpha$ [cf. Proposition 3.1, (ii)]. If the capsule of $\mathcal{D}^{\vdash}$-prime-strips $\left\{\alpha \mathfrak{D}^{\vdash}\right\}_{\alpha \in A}$ arises from a capsule of $\mathcal{F}^{\vdash \times \boldsymbol{\mu}}$-prime-strips

$$
\left\{{ }^{\alpha} \mathfrak{F}^{\vdash \times \boldsymbol{\mu}}\right\}_{\alpha \in A}=\left\{\left\{^{\alpha} \mathcal{F}_{\underline{v}}^{\vdash \times \mu}\right\}_{\underline{v} \in \underline{\mathbb{V}}}\right\}_{\alpha \in A}
$$

[relative to the given initial $\Theta$-data - cf. [IUTchI], §0; [IUTchII], Definition 4.9, (vii)], then we shall use similar notation to the notation just introduced concerning $\left\{{ }^{\alpha} \mathfrak{D}^{\vdash}\right\}_{\alpha \in A}$ to denote objects associated to $\left\{{ }^{\alpha} \mathfrak{F}^{\vdash \times \mu}\right\}_{\alpha \in A}$, i.e., by replacing " ${ }^{\vdash}$ " in the above notational conventions by " $\mathcal{F}^{\vdash \times \mu}$ " [cf. also the notation of Proposition 1.2, (vi), (vii)]. Then:

(i) (Mono-analytic/Holomorphic Compatibility) Suppose that the capsule of $\mathcal{D}^{\vdash}$-prime-strips $\left\{{ }^{\alpha} \mathfrak{D}^{\vdash}\right\}_{\alpha \in A}$ arises from the capsule of $\mathcal{F}$-prime-strips $\left\{{ }^{\alpha} \mathfrak{F}\right\}_{\alpha \in A}$ of Proposition 3.1; write $\left\{{ }^{\alpha} \mathfrak{F}^{\vdash \times \boldsymbol{\mu}}\right\}_{\alpha \in A}$ for the capsule of $\mathcal{F}^{\vdash \times \boldsymbol{\mu}}$-prime-strips associated to $\left\{{ }^{\alpha} \mathfrak{F}\right\}_{\alpha \in A}$. Then the poly-isomorphisms "log $\left({ }^{\dagger} \mathcal{D}_{\underline{v}}^{+}\right) \stackrel{\sim}{\rightarrow} \underline{\mathfrak{l o g}}\left({ }^{\dagger} \mathcal{F}_{\underline{v}}^{+} \times \boldsymbol{\mu}\right) \stackrel{\sim}{\rightarrow} \underline{\log }\left({ }^{\dagger} \mathcal{F}_{\underline{v}}\right)$ " of Proposition 1.2, (vi), (vii), induce natural poly-isomorphisms of topological modules

$$
\begin{gathered}
\underline{\log }\left({ }^{\alpha} \mathcal{D}_{v_{\mathbb{Q}}}^{\vdash}\right) \stackrel{\sim}{\rightarrow} \underline{\log }\left({ }^{\alpha} \mathcal{F}_{v_{\mathbb{Q}}}^{\vdash \times \boldsymbol{\mu}}\right) \stackrel{\sim}{\rightarrow} \underline{\log }\left({ }^{\alpha} \mathcal{F}_{v_{\mathbb{Q}}}\right) ; \underline{\log }\left({ }^{A} \mathcal{D}_{v_{\mathbb{Q}}}^{\vdash}\right) \stackrel{\sim}{\rightarrow} \underline{\log }\left({ }^{A} \mathcal{F}_{v_{\mathbb{Q}}}^{\vdash \times \boldsymbol{\mu}}\right) \stackrel{\sim}{\rightarrow} \underline{\mathfrak{l o g}}\left({ }^{A} \mathcal{F}_{v_{\mathbb{Q}}}\right) \\
\underline{\mathfrak{l o g}}\left({ }^{A, \alpha} \mathcal{D}_{\underline{v}}^{\vdash}\right) \stackrel{\sim}{\rightarrow} \underline{\mathfrak{l o g}}\left({ }^{A, \alpha} \mathcal{F}_{\underline{v}}^{\vdash \times \mu}\right) \stackrel{\sim}{\rightarrow} \underline{\mathfrak{l o g}}\left({ }^{A, \alpha} \mathcal{F}_{\underline{v}}\right)
\end{gathered}
$$

between the various "mono-analytic" tensor packets of the present Proposition 3.2 and the "holomorphic" tensor packets of Proposition 3.1. 
(ii) (Integral Structures) If $\underline{\mathbb{V}} \ni \underline{v} \mid v_{\mathbb{Q}} \in \mathbb{V}_{\mathbb{Q}}^{\text {non }}$, then the mono-analytic log-shells " $\mathcal{I}_{\dagger \mathcal{D}_{\underline{v}}^{\vdash}}$ " of Proposition 1.2, (vi), determine topological submodules

$\mathcal{I}\left({ }^{\alpha} \mathcal{D}_{v_{\mathbb{Q}}}^{\vdash}\right) \subseteq \underline{\log }\left({ }^{\alpha} \mathcal{D}_{v_{\mathbb{Q}}}^{\vdash}\right) ; \quad \mathcal{I}\left({ }^{A} \mathcal{D}_{v_{\mathbb{Q}}}^{\vdash}\right) \subseteq \underline{\mathfrak{l o g}}\left({ }^{A} \mathcal{D}_{v_{\mathbb{Q}}}^{\vdash}\right) ; \quad \mathcal{I}\left({ }^{A, \alpha} \mathcal{D}_{\underline{v}}^{\vdash}\right) \subseteq \underline{\mathfrak{l o g}}\left({ }^{A, \alpha} \mathcal{D}_{\underline{v}}^{\vdash}\right)$

- which may be regarded as integral structures on the $\mathbb{Q}$-spans of these submodules. If $\underline{\mathbb{V}} \ni \underline{v} \mid v_{\mathbb{Q}} \in \mathbb{V}_{\mathbb{Q}}^{\text {arc }}$, then by regarding the mono-analytic log-shell " $\mathcal{I}_{\dagger} \mathcal{D}_{\underline{v}}^{\vdash}$ " of Proposition 1.2, (vii), as the "closed unit ball" of a Hermitian metric on “log $\left({ }^{\dagger} \mathcal{D}_{\underline{v}}^{\vdash}\right)$ ", and considering the induced direct sum Hermitian metric on $\underline{\log }\left({ }^{\alpha} \mathcal{D}_{v_{\mathbb{Q}}}^{\vdash}\right)$, together with the induced tensor product Hermitian metric on $\underline{\mathfrak{l o g}}\left({ }^{A} \mathcal{D}_{v_{\mathbb{Q}}}^{\vdash}\right)$, one obtains Hermitian metrics on $\underline{\log }\left({ }^{\alpha} \mathcal{D}_{v_{\mathbb{Q}}}^{\vdash}\right)$, $\underline{\mathfrak{l o g}}\left({ }^{A} \mathcal{D}_{v_{\mathbb{Q}}}^{\vdash}\right)$, and $\underline{\mathfrak{l o g}}\left({ }^{A, \alpha} \overline{\mathcal{D}_{\underline{v}}^{\vdash}}\right)$, whose associated unit closed balls

$\mathcal{I}\left({ }^{\alpha} \mathcal{D}_{v_{\mathbb{Q}}}^{\vdash}\right) \subseteq \underline{\log }\left({ }^{\alpha} \mathcal{D}_{v_{\mathbb{Q}}}^{\vdash}\right) ; \quad \mathcal{I}\left({ }^{A} \mathcal{D}_{v_{\mathbb{Q}}}^{\vdash}\right) \subseteq \underline{\log }\left({ }^{A} \mathcal{D}_{v_{\mathbb{Q}}}^{\vdash}\right) ; \quad \mathcal{I}\left({ }^{A, \alpha} \mathcal{D}_{\underline{v}}^{\vdash}\right) \subseteq \underline{\log }\left({ }^{A, \alpha} \mathcal{D}_{\underline{v}}^{\vdash}\right)$

may be regarded as integral structures on $\underline{\log }\left({ }^{\alpha} \mathcal{D}_{v_{\mathbb{Q}}}^{\vdash}\right)$, $\underline{\log }\left({ }^{A} \mathcal{D}_{v_{\mathbb{Q}}}^{\vdash}\right)$, and $\underline{\log }\left({ }^{A, \alpha} \mathcal{D}_{\underline{v}}^{\vdash}\right)$, respectively. For arbitrary $\underline{\mathbb{V}} \ni \underline{v} \mid v_{\mathbb{Q}} \in \mathbb{V}_{\mathbb{Q}}$, we shall denote by " $\mathcal{I} \mathbb{Q}((-))$ " the $\bar{Q}$ span of "I $((-))$ "; also, we shall apply this notation involving " $\mathcal{I}((-))$ ", " $\mathcal{I}((-))$ "

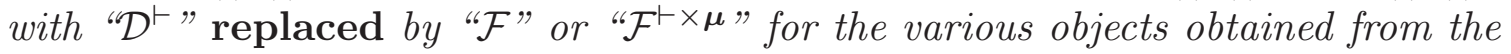
" $\mathcal{D}^{\vdash}$-versions" discussed above by applying the natural poly-isomorphisms of (i).

Proof. The various assertions of Proposition 3.2 follow immediately from the definitions and the references quoted in the statements of these assertions.

Remark 3.2.1. The issue of estimating the discrepancy between the holomorphic integral structures of Proposition 3.1, (ii), and the mono-analytic integral structures of Proposition 3.2, (ii), will form one of the main topics to be discussed in [IUTchIV] — cf. also Remark 3.9.1 below.

Remark 3.2.2. The constructions involving local mono-analytic tensor packets given in Proposition 3.2 may be applied to the capsules that appear in the various $\mathcal{D}^{\vdash}$-prime-strip processions - i.e., mono-analytic processions discussed in [IUTchI], Proposition 4.11, (ii); [IUTchI], Proposition 6.9, (ii).

\section{Proposition 3.3. (Global Tensor Packets) Let}

$$
{ }^{\dagger} \mathcal{H} \mathcal{T}^{\Theta^{ \pm \mathrm{ell}} \mathrm{NF}}
$$

be a $\Theta^{ \pm \text {ell }} \mathbf{N F - H o d g e ~ t h e a t e r ~ [ r e l a t i v e ~ t o ~ t h e ~ g i v e n ~ i n i t i a l ~} \Theta$-data] - cf. [IUTchI], Definition 6.13, (i). Thus, ${ }^{\dagger} \mathcal{H} \mathcal{T}^{\Theta^{ \pm e l l} \mathrm{NF}}$ determines $\Theta N F$ - and $\Theta^{ \pm \text {ell }}$-Hodge theaters ${ }^{\dagger} \mathcal{H} \mathcal{T}^{\Theta N F},{ }^{\dagger} \mathcal{H} \mathcal{T}^{\Theta^{ \pm e l l}}$ as in [IUTchII], Corollary 4.8. Let $\left\{{ }^{\alpha} \mathfrak{F}\right\}_{\alpha \in A}$ be an $n$-capsule of $\mathcal{F}$-prime-strips as in Proposition 3.1. Suppose, further, that $A$ is a subset of the index set $J$ that appears in the $\Theta N F$-Hodge theater ${ }^{\dagger} \mathcal{H} \mathcal{T}^{\Theta N F}$, and that, for each $\alpha \in A$, we are given a log-link

$$
\alpha \mathfrak{F} \quad \stackrel{\log }{\longrightarrow} \dagger \mathfrak{F}_{\alpha}
$$


- i.e., a poly-isomorphism of $\mathcal{F}$-prime-strips $\log \left({ }^{\alpha} \mathfrak{F}\right) \stackrel{\sim}{\rightarrow}{ }^{\dagger} \mathfrak{F}_{\alpha}$ [cf. Definition 1.1, (iii)]. Next, recall the field ${ }^{\dagger} \overline{\mathbb{M}}_{\text {mod }}^{\circ}$ discussed in [IUTchII], Corollary 4.8, (i); thus, [cf. [IUTchII], Corollary 4.8, (ii)], one also has, for $j \in J$, a labeled version $\left({ }^{\dagger} \overline{\mathbb{M}}_{\text {mod }}^{\ominus}\right)_{j}$ of this field. We shall refer to

$$
\left({ }^{\dagger} \overline{\mathbf{M}}_{\text {mod }}^{\odot}\right)_{A} \stackrel{\text { def }}{=} \bigotimes_{\alpha \in A}\left({ }^{\dagger} \overline{\mathbb{M}}_{\text {mod }}^{\odot}\right)_{\alpha}
$$

- where the tensor product is to be understood as a tensor product of modules as the global [n-]tensor packet associated to the subset $A \subseteq J$ and the $\Theta^{ \pm \text {ell }} N F$ Hodge theater ${ }^{\dagger} \mathcal{H} \mathcal{T}^{\Theta^{ \pm \mathrm{ell}} \mathrm{NF}}$.

(i) (Ring Structures) The field structure on the various $\left({ }^{\dagger} \overline{\mathbb{M}}_{\text {mod }}^{\odot}\right)_{\alpha}$, for $\alpha \in A$, determine a ring structure on $\left({ }^{\dagger} \overline{\mathbb{M}}_{\text {mod }}^{\odot}\right)_{A}$ with respect to which $\left({ }^{\dagger} \overline{\mathbb{M}}_{\text {mod }}^{\odot}\right)_{A}$ decomposes, uniquely, as a direct sum of number fields. Moreover, the various localization functors " $\left({ }^{\dagger} \mathcal{F}_{\text {mod }}^{\odot}\right)_{j} \rightarrow{ }^{\dagger} \mathfrak{F}_{j}$ " considered in [IUTchII], Corollary 4.8, (iii), determine, by composing with the given log-links, a natural injective localization ring homomorphism

$$
\left({ }^{\dagger} \overline{\mathbb{M}}_{\text {mod }}^{\odot}\right)_{A} \rightarrow \underline{\mathfrak{l o g}}\left({ }^{A} \mathcal{F}_{\mathbb{V}_{\mathbb{Q}}}\right) \stackrel{\text { def }}{=} \prod_{v_{\mathbb{Q}} \in \mathbb{V}_{\mathbb{Q}}} \underline{\log }\left({ }^{A} \mathcal{F}_{v_{\mathbb{Q}}}\right)
$$

to the product of the local holomorphic tensor packets considered in Proposition 3.1.

(ii) (Integral Structures) Fix an element $\alpha \in A$. Then by forming the tensor product with " 1 's" in the factors labeled by $\beta \in A \backslash\{\alpha\}$, one obtains a natural ring homomorphism

$$
\left({ }^{\dagger} \overline{\mathbb{M}}_{\text {mod }}^{\odot}\right)_{\alpha} \rightarrow\left({ }^{\dagger} \overline{\mathbb{M}}_{\text {mod }}^{\odot}\right)_{A}
$$

that induces an isomorphism of the domain onto a subfield of each of the direct summand number fields of the codomain. For each $v_{\mathbb{Q}} \in \mathbb{V}_{\mathbb{Q}}$, this homomorphism is compatible, in the evident sense, relative to the localization homomorphism of (i), with the natural homomorphism of topological rings considered in Proposition 3.1, (ii). Moreover, for each $v_{\mathbb{Q}} \in \mathbb{V}_{\mathbb{Q}}^{\text {non }}$, the composite of the above displayed homomorphism with the component at $v_{\mathbb{Q}}$ of the localization homomorphism of (i) maps the ring of integers of the number field $\left({ }^{\dagger} \overline{\mathrm{M}}_{\mathrm{mod}}^{\odot}\right)_{\alpha}$ into the submodule constituted by the integral structure on $\underline{\log }\left({ }^{A} \mathcal{F}_{v_{\mathbb{Q}}}\right)$ considered in Proposition 3.1, (ii); for each $v_{\mathbb{Q}} \in \mathbb{V}_{\mathbb{Q}}^{\text {arc }}$, the composite of the above displayed homomorphism with the component at $v_{\mathbb{Q}}$ of the localization homomorphism of (i) maps the set of archimedean integers [i.e., elements of absolute value $\leq 1$ at all archimedean primes] of the number field $\left({ }^{\dagger} \overline{\mathbb{M}}_{\text {mod }}^{\odot}\right)_{\alpha}$ into the direct product of subsets constituted by the integral structures considered in Proposition 3.1, (ii), on the various direct summand topological fields of $\underline{\log }\left({ }^{A} \mathcal{F}_{v_{\mathbb{Q}}}\right)$.

Proof. The various assertions of Proposition 3.3 follow immediately from the definitions and the references quoted in the statements of these assertions.

Remark 3.3.1. One may perform analogous constructions to the constructions of Proposition 3.3 for the fields " $\overline{\mathrm{M}}_{\text {mod }}^{\odot}\left({ }^{\dagger} \mathcal{D}^{\odot}\right)_{j}$ " of [IUTchII], Corollary 4.7, (ii) [cf. 
also the localization functors of [IUTchII], Corollary 4.7, (iii)], constructed from the associated $\mathcal{D}-\Theta^{ \pm \text {ell }} N F$-Hodge theater ${ }^{\dagger} \mathcal{H} \mathcal{T}^{\mathcal{D}-\Theta^{ \pm \text {ell }} \mathrm{NF}}$. These constructions are compatible with the corresponding constructions of Proposition 3.3, in the evident sense, relative to the various labeled Kummer-theoretic isomorphisms of [IUTchII], Corollary 4.8, (ii). We leave the routine details to the reader.

\section{Remark 3.3.2.}

(i) One may consider the image of the localization homomorphism of Proposition 3.3, (i), in the case of the various local holomorphic tensor packets arising from processions, as discussed in Remark 3.1.2. Indeed, at the level of the labels involved, this is immediate in the case of the "FF⿳亠丷⿵冂丶 Proposition 4.11, (i). On the other hand, in the case of the "| $\mathbb{F}_{l} \mid$-processions" of [IUTchI], Proposition 6.9, (i), this may be achieved by applying the identifying isomorphisms between the zero label $0 \in\left|\mathbb{F}_{l}\right|$ and the diagonal label $\left\langle\mathbb{F}_{l}^{*}\right\rangle$ associated to $\mathbb{F}_{l}^{*}$ discussed in [the final display of] [IUTchII], Corollary 4.6, (iii).

(ii) In a similar vein, one may compose the "D- $\Theta$ 土ell $N F$-Hodge theater version" discussed in Remark 3.3.1 of the localization homomorphism of Proposition 3.3, (i), with the product over $v_{\mathbb{Q}} \in \mathbb{V}_{\mathbb{Q}}$ of the inverses of the upper right-hand displayed isomorphisms at $v_{\mathbb{Q}}$ of Proposition 3.2, (i), and then consider the image of this composite morphism in the case of the various local mono-analytic tensor packets arising from processions, as discussed in Remark 3.2.2. Just as in the holomorphic case discussed in (i), in the case of the case of the "||Fㄴ $\mid$-processions" of [IUTchI], Proposition 6.9, (ii), this obliges one to apply the identifying isomorphisms between the zero label $0 \in\left|\mathbb{F}_{l}\right|$ and the diagonal label $\left\langle\mathbb{F}_{l}^{*}\right\rangle$ associated to $\mathbb{F}_{l}^{*}$ discussed in [the final display of] [IUTchII], Corollary 4.5, (iii).

(iii) The various images of global tensor packets discussed in (i) and (ii) above may be identified - i.e., in light of the injectivity of the homomorphisms applied to construct these images — with the global tensor packets themselves. These local holomorphic/local mono-analytic global tensor packet images will play a central role in the development of the theory of the present $\S 3$ [cf., e.g., Proposition 3.7 , below].

Remark 3.3.3. The log-shifted nature of the localization homomorphism of Proposition 3.3, (i), will play a crucial role in the development of the theory of present $\S 3$ - cf. the discussion of [IUTchII], Remark 4.8.2, (i), (iii).

Remark 3.3.4. Relative to the notation introduced in Propositions 3.1, 3.2, 3.3, write

$$
\underline{\log }_{\text {orb }}\left({ }^{\alpha}(-)_{\underline{v}}\right)
$$

— i.e., where "(-)" may be taken to be " $\mathcal{F}$ ", " $\mathcal{D}^{\vdash}$ ", or " $\mathcal{F}{ }^{\vdash \times \mu}$ " — for the set of

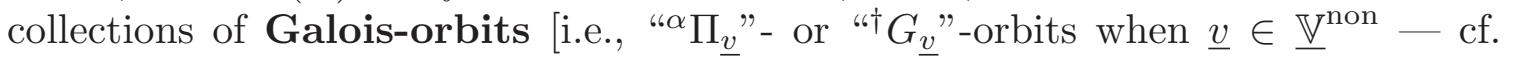
Propositions 1.2, (vi), and 3.1, (i); $\{1\}$-orbits, when $\left.\underline{v} \in \underline{\mathbb{V}}^{\text {arc }}\right]$ of $\underline{\log }\left({ }^{\alpha}(-)_{\underline{v}}\right)[$ cf. the definition of " $\mathbb{M}_{\text {orb }}^{\odot}(-)$ ", " $\overline{\mathbb{M}}_{\text {orb }}^{\odot}(-)$ " in [IUTchII], Corollary 4.7, (i) ];

$\underline{\mathfrak{l o g}}_{\mathrm{orb}}\left({ }^{\alpha}(-)_{v_{\mathbb{Q}}}\right) \stackrel{\text { def }}{=} \prod_{\underline{\mathbb{V}} \ni \underline{v} \mid v_{\mathbb{Q}}} \underline{\mathfrak{l o g}_{\mathrm{orb}}}\left({ }^{\alpha}(-)_{\underline{v}}\right) ; \quad \underline{\mathfrak{l o g}_{\mathrm{orb}}}\left({ }^{\alpha}(-)_{\mathbb{V}_{\mathbb{Q}}}\right) \stackrel{\text { def }}{=} \prod_{v_{\mathbb{Q}} \in \mathbb{V}_{\mathbb{Q}}} \underline{\mathfrak{l o g}_{\mathrm{orb}}}\left({ }^{\alpha}(-)_{v_{\mathbb{Q}}}\right)$ 


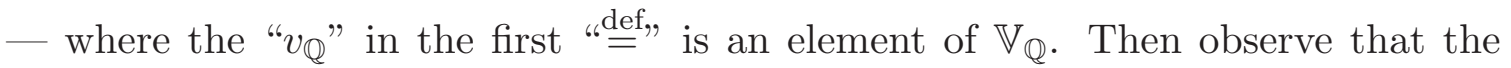
natural localization homomorphism

$$
\left({ }^{\dagger} \overline{\mathbb{M}}_{\text {mod }}^{\odot}\right)_{\alpha} \rightarrow \underline{\mathfrak{l o g}}\left({ }^{\alpha} \mathcal{F}_{\mathbb{V}_{\mathbb{Q}}}\right) \stackrel{\text { def }}{=} \prod_{v_{\mathbb{Q}} \in \mathbb{V}_{\mathbb{Q}}} \underline{\underline{l o g}}\left({ }^{\alpha} \mathcal{F}_{v_{\mathbb{Q}}}\right)
$$

[cf. Proposition 3.3, (i)] admits a natural Galois-orbit-theoretic extension

$$
\left(\left(\left(^{\dagger} \mathbb{M}_{\text {orb }}^{\odot}\right)_{\alpha} \subseteq\right) \quad\left({ }^{\dagger} \overline{\mathbb{M}}_{\text {orb }}^{\odot}\right)_{\alpha} \quad \rightarrow \quad \underline{\mathfrak{l o g}}_{\text {orb }}\left({ }^{\alpha} \mathcal{F}_{\mathbb{V}_{\mathbb{Q}}}\right)\right.
$$

[cf. the notation of [IUTchII], Corollary 4.8, (ii)] — i.e., the map of sets that assigns to a collection of global Galois-orbits the resulting collection, at each $\underline{v} \in \underline{\mathbb{V}}$, of local Galois-orbits.

$$
\begin{aligned}
& \underline{\underline{q}}^{1} \curvearrowright \quad \underline{\underline{q}}^{j^{2}} \curvearrowright \quad \underline{\underline{q}}^{\left(l^{*}\right)^{2} \curvearrowright} \\
& /^{ \pm} \hookrightarrow /^{ \pm} / \pm \hookrightarrow \ldots \hookrightarrow /^{ \pm} /{ }^{ \pm} \ldots /^{ \pm} \hookrightarrow{ }^{ \pm} \ldots \hookrightarrow /^{ \pm} / \pm \ldots \ldots /^{ \pm} \\
& \mathbb{S}_{1}^{ \pm} \quad \mathbb{S}_{1+1=2}^{ \pm} \quad \mathbb{S}_{j+1}^{ \pm} \quad \mathbb{S}_{1+l^{*}=l^{ \pm}}^{ \pm}
\end{aligned}
$$

Fig. 3.1: Splitting monoids of LGP-monoids acting on tensor packets

\section{Proposition 3.4. (Local Packet-theoretic Frobenioids)}

(i) (Single Packet Monoids) In the situation of Proposition 3.1, fix elements $\alpha \in A, \underline{v} \in \underline{\mathbb{V}}, v_{\mathbb{Q}} \in \mathbb{V}_{\mathbb{Q}}$ such that $\underline{v} \mid v_{\mathbb{Q}}$. Then by forming the image via the natural homomorphism $\underline{\log }\left({ }^{\alpha} \mathcal{F}_{\underline{v}}\right) \rightarrow \underline{\mathfrak{l o g}}\left({ }^{A, \alpha} \mathcal{F}_{\underline{v}}\right)$ of Proposition 3.1, (ii), the monoid $\Psi_{\log \left({ }^{\alpha} \mathcal{F}_{\underline{v}}\right)}$ [cf. the notation of Definition 1.1, (i), (ii)], together with its submonoid of units $\Psi_{\mathfrak{l o g}\left(\alpha \mathcal{F}_{\underline{v}}\right)}^{\times}$and realification $\Psi_{\mathfrak{l o g}\left(\alpha \mathcal{F}_{\underline{v}}\right)}^{\mathbb{R}}$, determine monoids

$$
\Psi_{\mathfrak{l o g}\left(A, \alpha \mathcal{F}_{\underline{v}}\right)}, \quad \Psi_{\mathfrak{l o g}\left(A, \alpha \mathcal{F}_{\underline{v}}\right)}^{\times}, \quad \Psi_{\mathfrak{l o g}\left(A, \alpha \mathcal{F}_{\underline{v}}\right)}^{\mathbb{R}}
$$

- which are equipped with $G_{\underline{v}}\left({ }^{\alpha} \Pi_{\underline{v}}\right)$-actions when $\underline{v} \in \underline{\mathbb{V}}^{\text {non }}$ and, in the case of the first displayed monoid, with a pair consisting of an Aut-holomorphic orbispace and a Kummer structure when $\underline{v} \in \underline{\mathbb{V}}^{\text {arc }}$. We shall think of these monoids as [possibly realified] subquotients of

$$
\log \left({ }^{A, \alpha} \mathcal{F}_{\underline{v}}\right)
$$

that act [multiplicatively] on appropriate [possibly realified] subquotients of $\log \left({ }^{A, \alpha} \mathcal{F}_{\underline{v}}\right)$. In particular, when $\underline{v} \in \underline{\mathbb{V}}^{\text {non }}$, the first displayed monoid, together with its ${ }^{\alpha} \Pi_{\underline{v}^{-}}$ action, determine a Frobenioid equipped with a natural isomorphism to ${ }^{\alpha} \mathcal{F}_{\underline{v}}$; when $\underline{v} \in \underline{\mathbb{V}}^{\text {arc }}$, the first displayed monoid, together with its Aut-holomorphic orbispace and Kummer structure, determine a collection of data equipped with a natural isomorphism to ${ }^{\alpha} \mathcal{F}_{\underline{v}}$.

(ii) (Local Logarithmic Gaussian Procession Monoids) Let

$$
{ }^{\ddagger} \mathcal{H} \mathcal{T}^{\Theta^{ \pm e l l} \mathrm{NF}} \quad \stackrel{\mathfrak{l o g}}{\longrightarrow}{ }^{\dagger} \mathcal{H} \mathcal{T}^{\Theta^{ \pm \mathrm{ell}} \mathrm{NF}}
$$


be a log-link of $\Theta^{ \pm \text {ell }} \mathbf{N F - H o d g e ~ t h e a t e r s ~ a s ~ i n ~ P r o p o s i t i o n ~ 1 . 3 , ~ ( i ) ~ [ c f . ~ a l s o ~ t h e ~}$ situation of Proposition 3.3]. Consider the $\mathcal{F}$-prime-strip processions that arise as the $\mathcal{F}$-prime-strip analogues [cf. Remark 3.1.2; [IUTchI], Remark 6.12.1] of the holomorphic processions discussed in [IUTchI], Proposition 6.9, (i), when the functor of [IUTchI], Proposition 6.9, (i), is applied to the $\Theta^{ \pm}$-bridges associated to ${ }^{\dagger} \mathcal{H} \mathcal{T}^{\Theta^{ \pm \mathrm{ell}} \mathrm{NF}},{ }_{\mathcal{H}} \mathcal{T}^{\Theta^{ \pm \mathrm{ell}} \mathrm{NF}}$; we shall refer to such processions as "†-" or " processions. Here, we recall that for $j \in\left\{1, \ldots, l^{*}\right\}$, the index set of the $(j+1)$ capsule that appear in such a procession is denoted $\mathbb{S}_{j+1}^{ \pm}$. Then by applying the various constructions of "single packet monoids" given in (i) in the case of the various capsules of $\mathcal{F}$-prime-strips that appear in a holomorphic $\ddagger$-procession - i.e., more precisely, in the case of the label $j \in\left\{1, \ldots, l^{*}\right\}$ [which shall occasionally identify with its image in $\left.\mathbb{F}_{l}^{*} \subseteq\left|\mathbb{F}_{l}\right|\right]$ that appears in the $(j+1)$-capsule of the $\ddagger$ procession - to the pull-backs, via the given log-link, of the [collections of] monoids $\Psi_{\mathcal{F}_{\text {gau }}}\left({ }^{\dagger} \mathcal{H} \mathcal{T}^{\Theta}\right)_{\underline{v}}, \infty \Psi_{\mathcal{F}_{\text {gau }}}\left({ }^{\dagger} \mathcal{H} \mathcal{T}^{\Theta}\right)_{\underline{v}}$ of [IUTchII], Corollary 4.6, (iv), for $\underline{v} \in \underline{\mathbb{V}}$, one obtains a functorial algorithm in the log-link of $\Theta^{ \pm \text {ell }} \mathbf{N F - H o d g e ~ t h e a t e r s ~}$ $\ddagger \mathcal{H} \mathcal{T}^{\Theta^{ \pm e l l} \mathrm{NF}} \stackrel{\text { log }}{\longrightarrow} \dagger \mathcal{H} \mathcal{T}^{\Theta^{ \pm \text {ell }} \mathrm{NF}}$ for constructing [collections of] monoids

$$
\underline{v} \ni \underline{\mathbb{V}} \mapsto \Psi_{\mathcal{F}_{\mathrm{LGP}}}\left({ }^{\dagger} \mathcal{H} \mathcal{T}^{\Theta^{ \pm \mathrm{ell}} \mathrm{NF}}\right)_{\underline{v}} ; \quad \underline{v} \ni \underline{\mathbb{V}} \mapsto \infty \Psi_{\mathcal{F}_{\mathrm{LGP}}}\left({ }^{\dagger} \mathcal{H} \mathcal{T}^{\Theta^{ \pm \mathrm{ell}} \mathrm{NF}}\right)_{\underline{v}}
$$

equipped with splittings [up to torsion, when $\underline{v} \in \underline{\mathbb{V}}^{\text {bad }}$ ] - which we refer to as "[local] LGP-monoids", or "logarithmic Gaussian procession monoids" [cf. Fig. 3.1 above]. Here, we note that the notation " $\left.{ }^{\dagger} \mathcal{H} \mathcal{T}^{\Theta^{ \pm \mathrm{ell}} \mathrm{NF}}\right)$ " constitutes a slight abuse of notation. Also, we note that this functorial algorithm requires one to apply the compatibility of the given log-link with the $\mathbb{F}_{l}^{\rtimes \pm}$-symmetrizing isomorphisms involved [cf. Remark 1.3.2]. For $\underline{v} \in \underline{\mathbb{V}}^{\text {bad }}$, the component labeled $j \in\left\{1, \ldots, l^{*}\right\}$ of the submonoid of Galois invariants [cf. (i)] of the entire LGP-monoid $\Psi_{\mathcal{F}_{\mathrm{LGP}}}\left({ }^{\dagger} \mathcal{H} \mathcal{T}^{\Theta^{ \pm \text {ell }} \mathrm{NF}}\right)_{\underline{v}}$ is a subset of

$$
\mathcal{I}^{\mathbb{Q}}\left(\mathbb{S}_{j+1}^{ \pm}, j ; \ddagger \mathcal{F}_{\underline{v}}\right)
$$

[i.e., where the notation "; $\neq$ " denotes the result of applying the discussion of (i) to the case of $\mathcal{F}$-prime-strips labeled " $\$$ "; cf. also the notational conventions of

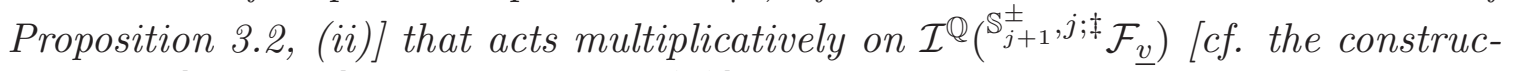
tions of [IUTchII], Corollary 3.6, (ii)]. For any $\underline{v} \in \mathbb{V}$, the component labeled $j \in\left\{1, \ldots, l^{*}\right\}$ of the submodule of Galois invariants [cf. (i) when $\underline{v} \in \underline{\mathbb{V}}^{\text {non }}$; this Galois action is trivial when $\left.\underline{v} \in \underline{\mathbb{V}}^{\text {arc }}\right]$ of the unit portion $\Psi_{\mathcal{F}_{\mathrm{LGP}}}\left({ }^{\dagger} \mathcal{H} \mathcal{T}^{{ }^{ \pm}}{ }^{ \pm \mathrm{ell}} \mathrm{NF}\right)_{\underline{v}}^{\times}$ of such an LGP-monoid is a subset of

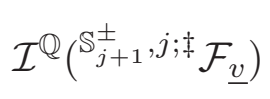

[cf. the discussion of (i); the notational conventions of Proposition 3.2, (ii)] that acts multiplicatively on $\mathcal{I}^{\mathbb{Q}}\left(\mathbb{S}_{j+1}^{ \pm}, j ; \ddagger \mathcal{F}_{\underline{v}}\right)$ [cf. the constructions of [IUTchII], Corollary 3.6, (ii); [IUTchII], Proposition 4.2, (iv); [IUTchII], Proposition 4.4, (iv)].

Proof. The various assertions of Proposition 3.4 follow immediately from the definitions and the references quoted in the statements of these assertions. 
Proposition 3.5. (Kummer Theory and Upper Semi-compatibility for Vertically Coric Local LGP-Monoids) Let $\left\{{ }^{n, m} \mathcal{H} \mathcal{T}^{\Theta^{ \pm e l l} \mathrm{NF}}\right\}_{n, m \in \mathbb{Z}}$ be a collection of distinct $\Theta^{ \pm \text {ell }} \mathbf{N F - H o d g e ~ t h e a t e r s ~ [ r e l a t i v e ~ t o ~ t h e ~ g i v e n ~ i n i t i a l ~} \Theta$-data] - which we think of as arising from a Gaussian log-theta-lattice [cf. Definition 1.4]. For each $n \in \mathbb{Z}$, write

$$
{ }^{n,{ }^{\circ}} \mathcal{H} \mathcal{T}^{\mathcal{D}-\Theta^{ \pm \mathrm{ell}} \mathrm{NF}}
$$

for the $\mathcal{D}-\Theta^{ \pm \text {ell }} N F$-Hodge theater determined, up to isomorphism, by the various ${ }^{n, m} \mathcal{H} \mathcal{T}^{\Theta^{ \pm e l l} \mathrm{NF}}$, where $m \in \mathbb{Z}$, via the vertical coricity of Theorem 1.5 , (i).

(i) (Vertically Coric Local LGP-Monoids and Associated Kummer Theory) Write

$$
\mathfrak{F}\left({ }^{n, \circ} \mathfrak{D}_{\succ}\right)_{t}
$$

for the $\mathcal{F}$-prime-strip associated [cf. [IUTchII], Remark 4.5.1, (i)] to the labeled collection of monoids " $\Psi_{\mathrm{cns}}\left({ }^{n, \circ} \mathfrak{D}_{\succ}\right)_{t}$ " of [IUTchII], Corollary 4.5, (iii) [i.e., where we take "t" to be "n, o"]. Recall the constructions of Proposition 3.4, (ii), involving $\mathcal{F}$-prime-strip processions. Then by applying these constructions to the $\mathcal{F}$-primestrips "FF( $\left.{ }^{n, 0} \mathfrak{D}_{\succ}\right)_{t}$ " and the various full log-links associated [cf. the discussion of Proposition 1.2, (ix)] to these $\mathcal{F}$-prime-strips - which we consider in a fashion compatible with the $\mathbb{F}_{l}^{\rtimes \pm}$-symmetries involved [cf. Remark 1.3.2; Proposition 3.4, (ii)] - we obtain a functorial algorithm in the $\mathcal{D}-\Theta^{ \pm e l l} \mathbf{N F}-H o d g e$ theater ${ }^{n, \circ} \mathcal{H} \mathcal{T}^{\mathcal{D}-\Theta^{ \pm \mathrm{ell}} \mathrm{NF}}$ for constructing [collections of] monoids

$$
\underline{v} \ni \underline{\mathbb{V}} \mapsto \Psi_{\mathrm{LGP}}\left({ }^{n, \circ} \mathcal{H} \mathcal{T}^{\mathcal{D}-\Theta^{ \pm \mathrm{ell}} \mathrm{NF}}\right)_{\underline{v}} ; \quad \underline{v} \ni \underline{\mathbb{V}} \mapsto{ }_{\infty} \Psi_{\mathrm{LGP}}\left({ }^{n, \circ} \mathcal{H} \mathcal{T}^{\mathcal{D}-\Theta^{ \pm \mathrm{ell}} \mathrm{NF}}\right)_{\underline{v}}
$$

equipped with splittings [up to torsion, when $\underline{v} \in \underline{\mathbb{V}}^{\text {bad }}$ ] - which we refer to as "vertically coric [local] LGP-monoids". For each $n, m \in \mathbb{Z}$, this functorial algorithm is compatible [in the evident sense] with the functorial algorithm of Proposition 3.4, (ii) - i.e., where we take "†" to be " $n, m$ " and "f" to be " $n, m-1$ " - relative to the Kummer isomorphisms of labeled data

$$
\Psi_{\mathrm{cns}}\left({ }^{n, m^{\prime}} \mathfrak{F}_{\succ}\right)_{t} \stackrel{\sim}{\rightarrow} \Psi_{\mathrm{cns}}\left({ }^{n, \circ} \mathfrak{D}_{\succ}\right)_{t}
$$

of [IUTchII], Corollary 4.6, (iii), and the evident identification, for $m^{\prime}=m, m-1$, of $^{n, m^{\prime}} \mathfrak{F}_{t}$ [i.e., the $\mathcal{F}$-prime-strip that appears in the associated $\Theta^{ \pm}$-bridge] with the $\mathcal{F}$-prime-strip associated to $\Psi_{\text {cns }}\left({ }^{n, m^{\prime}} \mathfrak{F}_{\succ}\right)_{t}$. In particular, for each $n, m \in \mathbb{Z}$, we obtain Kummer isomorphisms of [collections of] monoids

$$
\begin{aligned}
& \Psi_{\mathcal{F}_{\mathrm{LGP}}}\left({ }^{n, m} \mathcal{H} \mathcal{T}^{\Theta^{ \pm \mathrm{ell}} \mathrm{NF}}\right)_{\underline{v}} \stackrel{\sim}{\rightarrow} \Psi_{\mathrm{LGP}}\left({ }^{n, \circ} \mathcal{H} \mathcal{T}^{\mathcal{D}-\Theta^{ \pm \mathrm{ell}} \mathrm{NF}}\right)_{\underline{v}} \\
& \infty \Psi_{\mathcal{F}_{\mathrm{LGP}}}\left({ }^{n, m} \mathcal{H} \mathcal{T}^{\Theta^{ \pm \mathrm{ell}} \mathrm{NF}}\right)_{\underline{v}} \stackrel{\sim}{\rightarrow} \infty \Psi_{\mathrm{LGP}}\left({ }^{n, o} \mathcal{H} \mathcal{T}^{\mathcal{D}-\Theta^{ \pm \mathrm{ell}} \mathrm{NF}}\right)_{\underline{v}}
\end{aligned}
$$

for $\underline{v} \in \underline{\mathbb{V}}$.

(ii) (Upper Semi-compatibility) The Kummer isomorphisms of the final two displays of (i) are "upper semi-compatible" — cf. the discussion of "upper semi-commutativity" in Remark 1.2.2, (iii) - with the various log-links of $\Theta^{ \pm \text {ell }}$ NF-Hodge theaters ${ }^{n, m-1} \mathcal{H} \mathcal{T}^{\Theta^{ \pm e l l} \mathrm{NF}} \stackrel{\text { log }}{\longrightarrow}{ }^{n, m} \mathcal{H} \mathcal{T}^{\Theta^{ \pm e l l}} \mathrm{NF}$ [where $m \in \mathbb{Z}$ ] 
of the Gaussian log-theta-lattice under consideration in the following sense. Let $j \in\{0,1, \ldots, l *\}$. Then:

(a) (Nonarchimedean Primes) For $v_{\mathbb{Q}} \in \mathbb{V}_{\mathbb{Q}}^{\text {non }}$, the topological module

$$
\mathcal{I}\left({ }^{\mathbb{S}_{j+1}^{ \pm}} \mathcal{F}\left({ }^{n, \circ} \mathfrak{D}_{\succ}\right)_{v_{\mathbb{Q}}}\right)
$$

- i.e., that arises from applying the constructions of Proposition 3.4, (ii), in the vertically coric context of (i) above [cf. also the notational conventions of Proposition 3.2, (ii)] — contains the image of the submodules of Galois invariants [where we recall the Galois actions that appear in the data of [IUTchII], Corollary 4.6, (i), (iii)] of the groups of units $\left(\Psi_{\mathrm{cns}}\left({ }^{n, m} \mathfrak{F}_{\succ}\right)_{|t|}\right)_{v}^{\times}$, for $\underline{\mathbb{V}} \ni \underline{v} \mid v_{\mathbb{Q}}$ and $|t| \in\{0, \ldots, j\}$, via both

(1) the tensor product, over such $|t|$, of the [relevant] Kummer isomorphisms of $(i)$, and

(2) the tensor product, over such $|t|$, of the pre-composite of these Kummer isomorphisms with the $m^{\prime}$-th iterates of the log-links, for $m^{\prime} \geq 1$, of the $n$-th column of the Gaussian log-theta-lattice under consideration [cf. the discussion of Remark 1.2.2, (i), (iii)].

(b) (Archimedean Primes) For $v_{\mathbb{Q}} \in \mathbb{V}_{\mathbb{Q}}^{\text {arc }}$, the closed unit ball

$$
\mathcal{I}\left({ }^{\mathbb{S}_{j+1}^{ \pm}} \mathcal{F}\left({ }^{n, \circ} \mathfrak{D}_{\succ}\right)_{v_{\mathbb{Q}}}\right)
$$

- i.e., that arises from applying the constructions of Proposition 3.4, (ii), in the vertically coric context of (i) above [cf. also the notational conventions of Proposition 3.2, (ii)] - contains the image, via the tensor product, over $|t| \in\{0, \ldots, j\}$, of the [relevant] Kummer isomorphisms of (i), of both

(1) the groups of units $\left(\Psi_{\mathrm{cns}}\left(n, m \mathfrak{F}_{\succ}\right)_{|t|}\right)_{\underline{v}}^{\times}$, for $\underline{\mathbb{V}} \ni \underline{v} \mid v_{\mathbb{Q}}$, and

(2) the closed balls of radius $\pi$ inside $\left(\Psi_{\mathrm{cns}}\left({ }^{n, m} \mathfrak{F}_{\succ}\right)|t|\right) \underline{\underline{\mathrm{gp}}}[c f$. the notational conventions of Definition 1.1], for $\underline{\mathbb{V}} \ni \underline{v} \mid \bar{v}_{\mathbb{Q}}$.

Here, we recall from the discussion of Remark 1.2.2, (ii), (iii), that a closed ball as in (2) contains, for each $m^{\prime} \geq 1$, a subset that surjects, via the $m^{\prime}$-th iterate of the log-link of the $n$-th column of the Gaussian log-theta-lattice under consideration, onto the subset of the group of units $\left(\Psi_{\mathrm{cns}}\left(n, m-m^{\prime} \mathfrak{F}_{\succ}\right)_{|t|}\right)_{\underline{v}}^{\times}$given by the image, via this iterate, of the entire domain on which this iterate is defined.

(c) (Bad Primes) Let $\underline{v} \in \underline{\mathbb{V}}^{\text {bad }}$; suppose that $j \neq 0$. Recall that the various monoids " $\Psi_{\mathcal{F}_{\mathrm{LGP}}}(-)_{\underline{v}}$ ", " $\infty \Psi_{\mathcal{F}_{\mathrm{LGP}}}(-)_{\underline{v}}$ " constructed in Proposition 3.4, (ii), as well as the monoids " $\Psi_{\mathrm{LGP}}(-)_{\underline{v}}$ ", " $\infty \Psi_{\mathrm{LGP}}(-)_{\underline{v}}$ " constructed in (i) above, are equipped with natural splittings up to torsion. Write

$$
\Psi_{\mathcal{F}_{\mathrm{LGP}}}^{\perp}(-)_{\underline{v}} \subseteq \Psi_{\mathcal{F}_{\mathrm{LGP}}}(-)_{\underline{\underline{v}}} ; \quad \infty \Psi_{\mathcal{F}_{\mathrm{LGP}}}^{\perp}(-)_{\underline{v}} \subseteq \infty \Psi_{\mathcal{F}_{\mathrm{LGP}}}(-)_{\underline{v}}
$$




$$
\Psi_{\mathrm{LGP}}^{\perp}(-)_{\underline{v}} \subseteq \Psi_{\mathrm{LGP}}(-)_{\underline{v}} ; \quad \infty \Psi_{\mathrm{LGP}}^{\perp}(-)_{\underline{v}} \subseteq \infty \Psi_{\mathrm{LGP}}(-)_{\underline{v}}
$$

for the submonoids corresponding to these splittings [cf. the submonoids " $\mathcal{O}^{\perp}(-) \subseteq \mathcal{O}^{\triangleright}(-)$ " discussed in Definition 2.4, (i), in the case of " $\Psi^{\perp}$ "; the notational conventions of Theorem 2.2, (ii), in the case of " $\infty \Psi^{\perp}$ "]. [Thus, the subgroup of units of " $\Psi^{\perp}$ " consists of the $2 l$-torsion subgroup of " $\Psi$ ", while the subgroup of units of " $\infty \Psi^{\perp}$ " contains of the entire torsion torsion subgroup of " $\infty \Psi$ ".] Then, as $m$ ranges over the elements of $\mathbb{Z}$, the actions, via the [relevant] Kummer isomorphisms of (i), of the various monoids $\Psi_{\mathcal{F}_{\mathrm{LGP}}}^{\perp}\left({ }^{n, m} \mathcal{H} \mathcal{T}^{\Theta^{ \pm \mathrm{ell}} \mathrm{NF}}\right)_{\underline{v}} \subseteq \infty \Psi_{\mathcal{F}_{\mathrm{LGP}}}^{\perp}\left({ }^{n, m} \mathcal{H} \mathcal{T}^{\Theta^{ \pm \mathrm{ell}} \mathrm{NF}}\right)_{\underline{v}}$ on the topological modules

$$
\mathcal{I}^{\mathbb{Q}}\left(\mathbb{S}^{ \pm}{ }_{j+1}, j \mathcal{F}\left({ }^{n, \circ} \mathfrak{D}_{\succ}\right)_{\underline{v}}\right) \subseteq \underline{\mathfrak{l o g}}\left({ }^{\mathbb{S}_{j+1}^{ \pm}, j} \mathcal{F}\left({ }^{n, \circ} \mathfrak{D}_{\succ}\right)_{\underline{v}}\right)
$$

[where $\left.j=1, \ldots, l^{*}\right]$ - i.e., that arise from applying the constructions of Proposition 3.4, (ii), in the vertically coric context of (i) above [cf. also the notational conventions of Proposition 3.2, (ii)] — are mutually compatible, relative to the log-links of the $n$-th column of the Gaussian log-theta-lattice under consideration, in the sense that the only portions of these actions that are related to one another via these log-links are the indeterminacies with respect to multiplication by roots of unity, that is to say, indeterminacies at $m$ that correspond, via the log-link, to "addition by zero" - i.e., to no indeterminacy! - at $m+1$.

Now let us think of the various groups of units, for $\underline{v} \in \underline{\mathbb{V}}$,

$$
\left(\Psi_{\mathrm{cns}}\left({ }^{n, m} \mathfrak{F}_{\succ}\right)_{|t|}\right)_{\underline{v}}^{\times}, \quad \Psi_{\mathcal{F}_{\mathrm{LGP}}}\left(n, m \mathcal{H} \mathcal{T}^{\Theta^{ \pm \mathrm{ell}} \mathrm{NF}}\right)_{\underline{v}}^{\times}
$$

and splitting monoids, for $\underline{v} \in \underline{\mathbb{V}}^{\mathrm{bad}}$,

$$
\Psi_{\mathcal{F}_{\mathrm{LGP}}}^{\perp}\left({ }^{n, m} \mathcal{H} \mathcal{T}^{\Theta^{ \pm \mathrm{ell}} \mathrm{NF}}\right)_{\underline{v}}
$$

as acting on various portions of the modules, for $v_{\mathbb{Q}} \in \mathbb{V}_{\mathbb{Q}}$,

$$
\mathcal{I}^{\mathbb{Q}}\left(\mathbb{S}_{j+1}^{ \pm} \mathcal{F}\left({ }^{n, \circ} \mathfrak{D}_{\succ}\right)_{v_{\mathbb{Q}}}\right)
$$

not via a single Kummer isomorphism as in (i) - which fails to be compatible with the log-links of the Gaussian log-theta-lattice! - but rather via the totality of the various pre-composites of the relevant Kummer isomorphism with the various iterates of the log-links of the Gaussian log-theta-lattice - i.e., precisely as was described in detail in (a), (b), (c) above! Then one obtains a sort of "log-Kummer correspondence" between the totality, as $m$ ranges over the elements of $\mathbb{Z}$, of the various groups of units and splitting monoids just discussed [i.e., which are labeled by " $n, m$ "] and their actions on the " $\mathcal{Q}$ " labeled by " $n, \circ$ " which is invariant with respect to the translation symmetries [cf. Proposition 1.3, (iv)] of the $n$-th column of the Gaussian log-theta-lattice [cf. the discussion of Remark 1.2.2, (iii)].

Proof. The various assertions of Proposition 3.5 follow immediately from the definitions and the references quoted in the statements of these assertions. 
Example 3.6. Concrete Representations of Global Frobenioids. Before proceeding, we pause to take a closer look at the Frobenioid " $\mathcal{F}_{\bmod }^{\odot}$ " of [IUTchI], Example 5.1, (iii), i.e., more concretely speaking, the Frobenioid of arithmetic line

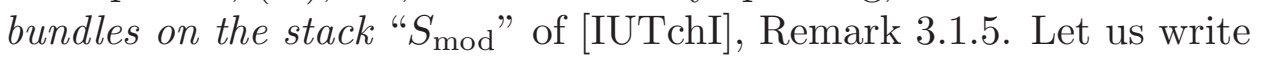

$$
\mathcal{F}_{\bmod }^{\odot}
$$

for the Frobenioid " $\mathcal{F}_{\bmod }^{\odot}$ " of [IUTchI], Example 5.1, (iii), in the case where the data denoted by the label "†" arises [in the evident sense] from data as discussed in [IUTchI], Definition 3.1. In the following discussion, we shall use the notation of [IUTchI], Definition 3.1.

(i) (Rational Function Torsor Version) For each $\underline{v} \in \underline{\mathbb{V}}$, the valuation on $K_{\underline{v}}$ determined by $\underline{v}$ determines a group homomorphism $\beta_{\underline{v}}: F_{\bmod }^{\times} \rightarrow K_{\underline{v}}^{\times} / \mathcal{O}_{K_{\underline{v}}}^{\times}[\mathrm{cf}$. Remark 3.6.1 below]. Then let us define a category $\mathcal{F}_{\text {MOD }}^{\odot}$ as follows. An object $\mathcal{T}=\left(T,\left\{t_{\underline{v}}\right\}_{\underline{v} \in \underline{\mathbb{V}}}\right)$ of $\mathcal{F}_{\text {MOD }}^{\odot}$ consists of

(a) an $F_{\bmod }^{\times}$-torsor $T$;

(b) a trivalization $t_{\underline{v}}$ of the torsor $T_{\underline{v}}$ obtained from $T$ by executing the "change of structure group" operation determined by the homomorphism $\beta_{\underline{v}}$.

An elementary morphism $\mathcal{T}_{1}=\left(T_{1},\left\{t_{1, \underline{v}}\right\}_{\underline{v} \in \underline{\mathbb{V}}}\right) \rightarrow \mathcal{T}_{2}=\left(T_{2},\left\{t_{2, \underline{v}}\right\}_{\underline{v} \in \underline{\mathbb{V}}}\right)$ between objects of $\mathcal{F}_{\text {MOD }}^{\odot}$ is defined to be an isomorphism $T_{1} \stackrel{\sim}{\rightarrow} T_{2}$ of $F_{\bmod }^{\times}$-torsors which is integral at each $\underline{v} \in \underline{\mathbb{V}}$, i.e., maps the trivialization $t_{1, \underline{v}}$ to an element of the $\mathcal{O}_{K_{\underline{v}}}^{\triangleright}$ orbit of $t_{2, \underline{v}}$. There is an evident notion of composition of elementary morphisms, as well as an evident notion of tensor powers $\mathcal{T}^{\otimes n}$, for $n \in \mathbb{Z}$, of an object $\mathcal{T}$ of $\mathcal{F}_{\text {MOD }}^{\odot}$. A morphism $\mathcal{T}_{1}=\left(T_{1},\left\{t_{1, \underline{v}}\right\}_{\underline{v} \in \underline{\mathbb{V}}}\right) \rightarrow \mathcal{T}_{2}=\left(T_{2},\left\{t_{2, \underline{v}}\right\}_{\underline{v} \in \underline{\mathbb{V}}}\right)$ between objects of $\mathcal{F}_{\text {MOD }}^{\odot}$ is defined to be an elementary morphism $\left(\mathcal{T}_{1}\right)^{\otimes n} \rightarrow \mathcal{T}_{2}$ for some positive integer $n$. There is an evident notion of composition of morphisms. Thus, $\mathcal{F}_{\text {MOD }}^{\odot}$ forms a category. In fact, one verifies immediately that, from the point of view of the theory of Frobenioids developed in [FrdI], [FrdII], $\mathcal{F}_{\text {MOD }}^{\odot}$ admits a natural Frobenioid structure [cf. [FrdI], Definition 1.3], for which the base category is the category with precisely one arrow. Relative to this Frobenioid structure, the elementary morphisms are precisely the linear morphisms, and the positive integer " $n$ " that appears in the definition of a morphism of $\mathcal{F}_{\mathrm{MOD}}^{\odot}$ is the Frobenius degree of the morphism. Moreover, by associating to an arithmetic line bundle on $S_{\bmod }$ the $F_{\bmod }^{\times}$ torsor determined by restricting the line bundle to the generic point of $S_{\bmod }$ and the local trivializations at $\underline{v} \in \underline{\mathbb{V}}$ determined by the various local integral structures, one verifies immediately that there exists a natural isomorphism of Frobenioids

$$
\mathcal{F}_{\text {mod }}^{\odot} \stackrel{\sim}{\rightarrow} \mathcal{F}_{\text {MOD }}^{\odot}
$$

that induces the identity morphism $F_{\mathrm{mod}}^{\times} \rightarrow F_{\mathrm{mod}}^{\times}$on the associated rational function monoids [cf. [FrdI], Corollary 4.10].

(ii) (Local Fractional Ideal Version) Let us define a category $\mathcal{F}_{\mathfrak{m o d}}^{\odot}$ as follows. An object

$$
\mathcal{J}=\left\{J_{\underline{v}}\right\}_{\underline{v} \in \underline{\mathbb{V}}}
$$


of $\mathcal{F}_{\mathfrak{m o d}}^{\odot}$ consists of a collection of "fractional ideals" $J_{v} \subseteq K_{v}$ for each $\underline{v} \in \underline{\mathbb{V}}$ - i.e., a finitely generated nonzero $\mathcal{O}_{K_{\underline{v}}}$-submodule of $K_{\underline{v}}$ when $\underline{v} \in \underline{\mathbb{V}}^{\text {non }}$; a positive real multiple of $\mathcal{O}_{K_{\underline{v}}} \stackrel{\text { def }}{=}\left\{\lambda \in K_{\underline{v}}|| \lambda \mid \leq 1\right\} \subseteq K_{\underline{v}}$ when $\underline{v} \in \underline{\mathbb{V}}^{\text {arc }}$ - such that $J_{\underline{v}}=\mathcal{O}_{K_{\underline{v}}}$ for all but finitely many $\underline{v}$. If $\mathcal{J}=\left\{J_{\underline{v}}\right\}_{\underline{v} \in \underline{\mathbb{V}}}$ is an object of $\mathcal{F}_{\mathfrak{m} \mathfrak{d} \mathfrak{d}}^{\odot}$, then for any element $f \in F_{\text {mod }}^{\times}$, one obtains an object $f \cdot \mathcal{J}=\left\{f \cdot J_{\underline{v}}\right\}_{\underline{v} \in \underline{\mathbb{V}}}$ of $\mathcal{F}_{\mathfrak{m o d}}^{\odot}$ by multiplying each of the fractional ideals $J_{\underline{v}}$ by $f$. Moreover, if $\mathcal{J}=\left\{J_{\underline{v}}\right\}_{\underline{v}} \in \underline{\mathbb{V}}$ is an object of $\mathcal{F}_{\mathfrak{m o d}}^{\odot}$, then for any $n \in \mathbb{Z}$, there is an evident notion of the $n$-th tensor power $\mathcal{J}^{\otimes n}$ of $\mathcal{J}$. An elementary morphism $\mathcal{J}_{1}=\left\{J_{1, v}\right\}_{v \in \mathbb{V}} \rightarrow \mathcal{J}_{2}=\left\{J_{2, v}\right\}_{v \in \mathbb{V}}$ between objects of $\mathcal{F}_{\text {mod }}^{\odot}$ is defined to be an element $f \in F_{\text {mod }}^{\times}$that is integral with respect to $\mathcal{J}_{1}$ and $\mathcal{J}_{2}$ in the sense that $f \cdot J_{1, \underline{v}} \subseteq J_{2, \underline{v}}$ for each $\underline{v} \in \underline{\mathbb{V}}$. There is an evident notion of composition of elementary morphisms. A morphism $\mathcal{J}_{1}=\left\{J_{1, \underline{v}}\right\}_{\underline{v} \in \underline{\mathbb{V}}} \rightarrow \mathcal{J}_{2}=\left\{J_{2, \underline{v}}\right\}_{\underline{v} \in \underline{\mathbb{V}}}$ between objects of $\mathcal{F}_{\mathfrak{m o d}}^{\odot}$ is defined to be an elementary morphism $\left(\mathcal{J}_{1}\right)^{\otimes n} \rightarrow \mathcal{J}_{2}$ for some positive integer $n$. There is an evident notion of composition of morphisms. Thus, $\mathcal{F}_{\mathfrak{m o d}}^{\odot}$ forms a category. In fact, one verifies immediately that, from the point of view of the theory of Frobenioids developed in $[\mathrm{FrdI}],[\mathrm{FrdII}], \mathcal{F}_{\mathfrak{m o d}}^{\odot}$ admits a natural Frobenioid structure [cf. [FrdI], Definition 1.3], for which the base category is the category with precisely one arrow. Relative to this Frobenioid structure, the elementary morphisms are precisely the linear morphisms, and the positive integer " $n$ " that appears in the definition of a morphism of $\mathcal{F}_{\mathfrak{m o d}}^{\odot}$ is the Frobenius degree of the morphism. Moreover, by associating to an object $\mathcal{J}=\left\{J_{\underline{v}}\right\}_{\underline{v} \in \underline{\mathbb{V}}}$ of $\mathcal{F}_{\mathfrak{m o d}}^{\odot}$ the arithmetic line bundle on $S_{\text {mod }}$ obtained from the trivial arithmetic line bundle on $S_{\text {mod }}$ by modifying the integral structure of the trivial line bundle at $\underline{v} \in \underline{\mathbb{V}}$ in the fashion prescribed by $J_{\underline{v}}$, one verifies immediately that there exists a natural isomorphism of Frobenioids

$$
\mathcal{F}_{\mathfrak{m o d}}^{\odot} \stackrel{\sim}{\rightarrow} \mathcal{F}_{\bmod }^{\odot}
$$

that induces the identity morphism $F_{\mathrm{mod}}^{\times} \rightarrow F_{\bmod }^{\times}$on the associated rational function monoids [cf. [FrdI], Corollary 4.10].

(iii) By composing the isomorphisms of Frobenioids of (i) and (ii), one thus obtains a natural isomorphism of Frobenioids

$$
\mathcal{F}_{\mathfrak{m o d}}^{\odot} \stackrel{\sim}{\rightarrow} \mathcal{F}_{\mathrm{MOD}}^{\odot}
$$

that induces the identity morphism $F_{\text {mod }}^{\times} \rightarrow F_{\text {mod }}^{\times}$on the associated rational function monoids [cf. [FrdI], Corollary 4.10]. One verifies immediately that although the above isomorphism of Frobenioids is not necessarily determined by the condition that it induce the identity morphism on $F_{\text {mod }}^{\times}$, the induced isomorphism between the respective perfections [hence also on realifications] of $\mathcal{F}_{\mathfrak{m o d}}^{\odot}, \mathcal{F}_{\text {MOD }}^{\odot}$ is completely determined by this condition.

Remark 3.6.1. Note that, as far the various constructions of Example 3.6, (i), are concerned, the various homomorphisms $\beta_{\underline{v}}$, for $\underline{v} \in \underline{\mathbb{V}}$, may be thought of, alternatively, as a collection of

$$
\text { subquotients of the perfection }\left(F_{\mathrm{mod}}^{\times}\right)^{\mathrm{pf}} \text { of } F_{\mathrm{mod}}^{\times}
$$

- each of which is equipped with a submonoid of "nonnegative elements" - that are completely determined by the ring structure of the field $F_{\bmod }$ [i.e., equipped with its structure as the field of moduli of $X_{F}$ ]. 


\section{Remark 3.6.2.}

(i) In the theory to be developed below, we shall be interested in relating the realifications of various Frobenioids isomorphic to $\mathcal{F}_{\bmod }^{\odot}$ that lie on opposite sides of the $\Theta_{\mathrm{gau}}^{\times \boldsymbol{\mu}}$-link to one another. In particular, at the level of objects of the Frobenioids involved, it only makes sense to work with isomorphism classes of objects that are preserved by the isomorphisms of Frobenioids that appear. Here, we note that the isomorphism classes of the sort of Frobenioids that appear in this context are determined, in an essential way, by the rational function monoid of the Frobenioid in question [cf. the constructions given in [FrdI], Theorem 5.2, (i)]. In this context, we observe that the rational function monoid $F_{\text {mod }}^{\times}$of $\mathcal{F}_{\bmod }^{\odot}$ satisfies the following fundamental property:

[the union with $\{0\}$ of] $F_{\bmod }^{\times}$admits a natural additive structure.

In this context, we note that this property is not satisfied by

(a) the rational function monoids of the perfection or realification of $\mathcal{F}_{\bmod }^{\odot}$

(b) subgroups $\Gamma \subseteq F_{\text {mod }}^{\times}$- such as, for instance, the trivial subgroup $\{1\}$ or the subgroup of $S$-units, for $S \subseteq \mathbb{V}_{\bmod }$ a nonempty finite subset - that do not arise as the multiplicative group of some subfield of $F_{\bmod }$.

The significance of this fundamental property is that it allows one to represent the objects of $\mathcal{F}_{\text {mod }}^{\odot}$ additively, i.e., as modules - cf. the point of view of Example 3.6, (ii). At a more concrete level, if, in the notation of (b), one considers the result of "adding" two elements of a $\Gamma$-torsor [cf. the point of view of Example 3.6, (i)!], then the resulting "sum" can only be rendered meaningful, relative to the given $\Gamma$ torsor, if $\Gamma$ is additively closed. The additive representation of objects of $\mathcal{F}_{\bmod }^{\odot}$ will be of crucial importance in the theory of the present series of papers since it will allow us to relate objects of $\mathcal{F}_{\text {mod }}^{\odot}$ on opposite sides of the $\Theta_{\text {gau }}^{\times \mu}$-link to one another - which, a priori, are only related to one another at the level of realifications in a multiplicative fashion - by means of mono-analytic log-shells [cf. the discussion of [IUTchII], Remark 4.7.2].

(ii) One way to understand the content of the discussion of (i) is as follows: whereas

the construction of $\mathcal{F}_{\mathfrak{m} \mathfrak{o d}}^{\odot}$ depends on the additive structure of $F_{\bmod }^{\times}$

in an essential way,

the construction of $\mathcal{F}_{\mathrm{MOD}}^{\odot}$ is strictly multiplicative in nature.

Indeed, the construction of $\mathcal{F}_{\text {MOD }}^{\odot}$ given in Example 3.6, (i), is essentially the same as the construction of $\mathcal{F}_{\bmod }^{\odot}$ given in [FrdI], Example 6.3 [i.e., in effect, in [FrdI], Theorem 5.2, (i)]. From the this point of view, it is natural to identify $\mathcal{F}_{\text {MOD }}^{\odot}$ with $\mathcal{F}_{\text {mod }}^{\odot}$ via the natural isomorphism of Frobenioids of Example 3.6, (i). We shall often do this in the theory to be developed below. 


\section{Proposition 3.7. (Global Packet-theoretic Frobenioids)}

(i) (Single Packet Rational Function Torsor Version) In the notation of Proposition 3.3: For each $\alpha \in A$, there is an algorithm for constructing, as discussed in Example 3.6, (i) [cf. also Remark 3.6.1], from the [number] field given by the image

$$
\left({ }^{\dagger} \overline{\mathrm{M}}_{\mathrm{MOD}}^{\odot}\right)_{\alpha}
$$

of the composite

$$
\left({ }^{\dagger} \overline{\mathbb{M}}_{\text {mod }}^{\odot}\right)_{\alpha} \rightarrow\left({ }^{\dagger} \overline{\mathbb{M}}_{\text {mod }}^{\odot}\right)_{A} \rightarrow \underline{\log }\left({ }^{A} \mathcal{F}_{\mathbb{V}_{\mathbb{Q}}}\right)
$$

of the homomorphisms of Proposition 3.3, (i), (ii), a Frobenioid $\left({ }^{\dagger} \mathcal{F}_{\mathrm{MOD}}^{\odot}\right)_{\alpha}$, together with a natural isomorphism of Frobenioids

$$
\left({ }^{\dagger} \mathcal{F}_{\bmod }^{\odot}\right)_{\alpha} \stackrel{\sim}{\rightarrow}\left({ }^{\dagger} \mathcal{F}_{\mathrm{MOD}}^{\odot}\right)_{\alpha}
$$

[cf. the notation of [IUTchII], Corollary 4.8, (ii)] that induces the tautological isomorphism $\left({ }^{\dagger} \mathbb{M}_{\text {mod }}^{\odot}\right)_{\alpha} \stackrel{\sim}{\rightarrow}\left({ }^{\dagger} \mathbb{M}_{\text {MOD }}^{\odot}\right)_{\alpha}$ on the associated rational function monoids [cf. Example 3.6, (i)]. We shall often use this isomorphism of Frobenioids to identify $\left({ }^{\dagger} \mathcal{F}_{\bmod }^{\odot}\right)_{\alpha}$ with $\left({ }^{\dagger} \mathcal{F}_{\mathrm{MOD}}^{\odot}\right)_{\alpha}\left[c f\right.$. Remark 3.6.2, (ii)]. Write $\left({ }^{\dagger} \mathcal{F}_{\mathrm{MOD}}^{\odot \mathbb{R}}\right)_{\alpha}$ for the realification of $\left({ }^{\dagger} \mathcal{F}_{\mathrm{MOD}}^{\odot}\right)_{\alpha}$.

(ii) (Single Packet Local Fractional Ideal Version) In the notation of Propositions 3.3, 3.4: For each $\alpha \in A$, there is an algorithm for constructing, as discussed in Example 3.6, (ii), from the [number] field $\left({ }^{\dagger} \overline{\mathrm{M}}_{\mathfrak{m o d}}^{\odot}\right)_{\alpha} \stackrel{\text { def }}{=}\left({ }^{\dagger} \overline{\mathrm{M}}_{\mathrm{MOD}}^{\odot}\right)_{\alpha}[c f$. (i)] and the Galois invariants of the local monoids

$$
\Psi_{\mathfrak{l o g}\left(A, \alpha \mathcal{F}_{\underline{v}}\right)} \subseteq \log \left({ }^{A, \alpha} \mathcal{F}_{\underline{v}}\right)
$$

for $\underline{v} \in \mathbb{V}$ of Proposition 3.4, (i) - i.e., so the corresponding local "fractional ideal $J_{\underline{v}}$ " of Example 3.6, (ii), is a subset [indeed a submodule when $\underline{v} \in \underline{\mathbb{V}}^{\text {non }}$ ] of $\mathcal{I}^{\mathbb{Q}}\left(A, \alpha \mathcal{F}_{\underline{v}}\right)$ whose $\mathbb{Q}$-span is equal to $\mathcal{I}^{\mathbb{Q}}\left({ }^{A, \alpha} \mathcal{F}_{\underline{v}}\right)$ [cf. the notational conventions of Proposition 3.2, (ii)] - a Frobenioid $\left({ }^{\dagger} \mathcal{F}_{\mathfrak{m o d}}^{\odot}\right)_{\alpha}$, together with natural isomorphisms of Frobenioids

$$
\left({ }^{\dagger} \mathcal{F}_{\mathfrak{m o d}}^{\odot}\right)_{\alpha} \stackrel{\sim}{\rightarrow}\left({ }^{\dagger} \mathcal{F}_{\bmod }^{\odot}\right)_{\alpha} ; \quad\left({ }^{\dagger} \mathcal{F}_{\mathfrak{m o d}}^{\odot}\right)_{\alpha} \stackrel{\sim}{\rightarrow}\left({ }^{\dagger} \mathcal{F}_{\mathrm{MOD}}^{\odot}\right)_{\alpha}
$$

that induce the tautological isomorphisms $\left({ }^{\dagger} \mathbb{M}_{\mathfrak{m o d}}^{\odot}\right)_{\alpha} \stackrel{\sim}{\rightarrow}\left({ }^{\dagger} \mathbb{M}_{\bmod }^{\odot}\right)_{\alpha},\left({ }^{\dagger} \mathbb{M}_{\mathfrak{m o d}}^{\odot}\right)_{\alpha} \stackrel{\sim}{\rightarrow}$ $\left({ }^{\dagger} \mathbb{M}_{\mathrm{MOD}}^{\odot}\right)_{\alpha}$ on the associated rational function monoids [cf. the natural isomorphism of Frobenioids of (i); Example 3.6, (ii), (iii)]. Write $\left({ }^{\dagger} \mathcal{F}_{\mathfrak{m o d}}^{\odot \mathbb{R}}\right)_{\alpha}$ for the realification of $\left({ }^{\dagger} \mathcal{F}_{\mathfrak{m o d}}^{\odot}\right)_{\alpha}$.

(iii) (Realified Global LGP-Frobenioids) In the notation of Proposition 3.4: By applying the composites of the isomorphisms of Frobenioids " ${ }^{\mathbb{C}} \mathcal{C}_{j} \stackrel{\sim}{\rightarrow}$ $\left({ }^{\dagger} \mathcal{F}_{\bmod }^{\odot \mathbb{R}}\right)_{j}$ " of [IUTchII], Corollary 4.8, (iii), with the realifications " $\left({ }^{\dagger} \mathcal{F}_{\bmod }^{\odot \mathbb{R}}\right)_{\alpha} \stackrel{\sim}{\rightarrow}$ $\left({ }^{\dagger} \mathcal{F}_{\mathrm{MOD}}^{\odot \mathbb{R}}\right)_{\alpha}$ " of the isomorphisms of Frobenioids of (i) above to the realified global Frobenioid portion ${ }^{\dagger} \mathcal{C}_{\text {gau }}^{\Vdash}$ of the $\mathcal{F}^{\Vdash}$-prime-strip ${ }^{\dagger} \mathfrak{F}_{\text {gau }}^{\Vdash}$ of [IUTchII], Corollary 4.10, (ii) [cf. Remarks 1.5.3, (iii); 3.3.2, (i)], one obtains a functorial algorithm 
in the log-link of $\Theta^{ \pm \text {ell }}$ NF-Hodge theaters $\ddagger \mathcal{H} \mathcal{T}^{\Theta^{ \pm e l l}} \mathrm{NF} \stackrel{\mathfrak{l o g}}{\longrightarrow} \dagger \mathcal{H} \mathcal{T}^{\Theta^{ \pm e l l}} \mathrm{NF}$ of Proposition 3.4, (ii), for constructing a Frobenioid

$$
\mathcal{C}_{\mathrm{LGP}}^{\Vdash}\left({ }^{\dagger} \mathcal{H} \mathcal{T}^{\Theta^{ \pm \mathrm{ell}} \mathrm{NF}}\right)
$$

- which we refer to as a "global realified LGP-Frobenioid". Here, we note that the notation " $\left.{ }^{\dagger} \mathcal{H} \mathcal{T}^{\Theta^{ \pm \mathrm{ell}} \mathrm{NF}}\right)$ " constitutes a slight abuse of notation. In particular, the global realified Frobenioid ${ }^{\dagger} \mathcal{C}_{\mathrm{LGP}}^{\Vdash} \stackrel{\text { def }}{=} \mathcal{C}_{\mathrm{LGP}}^{\Vdash}\left({ }^{\dagger} \mathcal{H} \mathcal{T}^{\Theta^{ \pm \text {ell }} \mathrm{NF}}\right)$, together with the collection of data $\left.\Psi_{\mathcal{F}_{\mathrm{LGP}}}{ }^{\dagger} \mathcal{H} \mathcal{T}^{{ }^{\Theta}{ }^{ \pm e l l} \mathrm{NF}}\right)$ constructed in Proposition 3.4, (ii), give rise, in a natural fashion, to an $\mathcal{F}^{\Vdash}$-prime-strip

$$
{ }^{\dagger} \mathfrak{F}_{\mathrm{LGP}}^{\Vdash}=\left({ }^{\dagger} \mathcal{C}_{\mathrm{LGP}}^{\Vdash}, \operatorname{Prime}\left({ }^{\dagger} \mathcal{C}_{\mathrm{LGP}}^{\Vdash}\right) \stackrel{\sim}{\rightarrow} \underline{\mathbb{V}},{ }^{\dagger} \mathfrak{F}_{\mathrm{LGP}}^{\vdash}, \quad\left\{{ }^{\dagger} \rho_{\mathrm{LGP}, \underline{v}}\right\}_{\underline{v} \in \underline{\mathbb{V}}}\right)
$$

- cf. the construction of the $\mathcal{F}^{\Vdash}$-prime-strip ${ }^{\dagger} \mathfrak{F}_{\text {gau }}^{\Vdash}$ in [IUTchII], Corollary 4.10, (ii) - together with a natural isomorphism

$$
{ }^{\dagger} \mathfrak{F}_{\text {gau }}^{\Vdash} \stackrel{\sim}{\rightarrow} \dagger \mathfrak{F}_{\text {LGP }}^{\Vdash}
$$

of $\mathcal{F}^{\Vdash}$-prime-strips [i.e., that arises tautologically from the construction of ${ }^{\dagger} \mathfrak{F}_{\mathrm{LGP}}^{\Vdash !}$ !]

(iv) (Realified Global lgp-Frobenioids) In the situation of (iii) above, write $\Psi_{\mathcal{F}_{\mathfrak{l g p}}}\left({ }^{\dagger} \mathcal{H} \mathcal{T}^{\Theta^{ \pm \text {ell }} \mathrm{NF}}\right) \stackrel{\text { def }}{=} \Psi_{\mathcal{F}_{\mathrm{LGP}}}\left({ }^{\dagger} \mathcal{H} \mathcal{T}^{\Theta^{ \pm \text {ell }} \mathrm{NF}}\right),{ }^{\dagger} \mathfrak{F}_{\mathfrak{l g p}} \stackrel{\text { def }}{=}{ }^{\dagger} \mathfrak{F}_{\mathrm{LGP}}^{\leftarrow}$. Then by replacing, in the construction of (iii), the isomorphisms " $\left({ }^{\dagger} \mathcal{F}_{\bmod }^{\odot \mathbb{R}}\right)_{\alpha} \stackrel{\sim}{\rightarrow}\left({ }^{\dagger} \mathcal{F}_{\mathrm{MOD}}^{\odot \mathbb{R}}\right) \alpha$ " by the natural isomorphisms " $\left({ }^{\dagger} \mathcal{F}_{\mathrm{mod}}^{\odot \mathbb{R}}\right)_{\alpha} \stackrel{\sim}{\rightarrow}\left({ }^{\dagger} \mathcal{F}_{\mathfrak{m o d}}^{\odot \mathbb{R}}\right)_{\alpha}$ " [cf. (ii)], one obtains a functorial algorithm in the log-link of $\Theta^{ \pm \text {ell }} \mathbf{N F}$-Hodge theaters ${ }^{\ddagger} \mathcal{H} \mathcal{T}^{\Theta^{ \pm \mathrm{ell}} \mathrm{NF}} \stackrel{\text { log }}{\longrightarrow}{ }^{\dagger} \mathcal{H} \mathcal{T}^{\Theta^{ \pm \mathrm{ell}} \mathrm{NF}}$ of Proposition 3.4, (ii), for constructing a Frobenioid

$$
\mathcal{C}_{\mathfrak{l g p}}^{\Vdash}\left({ }^{\dagger} \mathcal{H} \mathcal{T}^{\Theta^{ \pm e l l} \mathrm{NF}}\right)
$$

- which we refer to as a "global realified lgp-Frobenioid" — as well as an $\mathcal{F}^{\Vdash}$-prime-strip

$$
{ }^{\dagger} \mathfrak{F}_{\mathfrak{l g} \mathfrak{p}}^{\vdash}=\left({ }^{\dagger} \mathcal{C}_{\mathfrak{l g} \mathfrak{p}}^{\Vdash}, \operatorname{Prime}\left({ }^{\dagger} \mathcal{C}_{\mathfrak{l g p}}^{\Vdash}\right) \stackrel{\sim}{\rightarrow} \underline{\mathbb{V}},{ }^{\dagger} \mathfrak{F}_{\mathfrak{l g} \mathfrak{p}}^{\vdash},\left\{{ }^{\dagger} \rho_{\mathfrak{l g} \mathfrak{p}, \underline{v}}\right\}_{\underline{v} \in \underline{\mathbb{V}}}\right)
$$

- where we write ${ }^{\dagger} \mathcal{C}_{\mathfrak{l g p}}^{\Vdash} \stackrel{\text { def }}{=} \mathcal{C}_{\mathfrak{l g p}}^{\Vdash}\left({ }^{\dagger} \mathcal{H} \mathcal{T}^{\Theta^{ \pm e l l} \mathrm{NF}}\right)$ - together with tautological isomorphisms

$$
\dagger \mathfrak{F}_{\text {gau }}^{\vdash} \stackrel{\sim}{\rightarrow} \quad \dagger \mathfrak{F}_{\text {LGP }}^{\vdash} \stackrel{\sim}{\rightarrow} \quad \dagger \mathfrak{F}_{\mathfrak{l g p}}^{\vdash}
$$

of $\mathcal{F}^{\Vdash}$-prime-strips [cf. (iii)].

(v) (Realified Product Embeddings and Non-realified Global Frobenioids) The constructions of $\mathcal{C}_{\mathrm{LGP}}^{\Vdash}\left({ }^{\dagger} \mathcal{H} \mathcal{T}^{\Theta^{ \pm e l l} \mathrm{NF}}\right), \mathcal{C}_{\mathfrak{l g p}}^{\Vdash}\left({ }^{\dagger} \mathcal{H} \mathcal{T}^{\Theta^{ \pm e l l}} \mathrm{NF}\right)$ given in (iii) and (iv) above give rise to a commutative diagram of categories

$$
\begin{array}{cccc}
\mathcal{C}_{\mathrm{LGP}}^{\Vdash}\left({ }^{\dagger} \mathcal{H} \mathcal{T}^{\Theta^{ \pm \mathrm{ell}} \mathrm{NF}}\right) & \hookrightarrow & \prod_{j \in \mathbb{F}_{l}^{*}}\left({ }^{\dagger} \mathcal{F}_{\mathrm{MOD}}^{\odot \mathbb{R}}\right)_{j} \\
\downarrow & & & \downarrow \\
\mathcal{C}_{\mathfrak{l g p}}^{\Vdash}\left({ }^{\dagger} \mathcal{H} \mathcal{T}^{\Theta^{ \pm \mathrm{ell}} \mathrm{NF}}\right) & \hookrightarrow & \prod_{j \in \mathbb{F}_{l}^{*}}\left({ }^{\dagger} \mathcal{F}_{\mathfrak{m o d}}^{\odot \mathbb{R}}\right)_{j}
\end{array}
$$

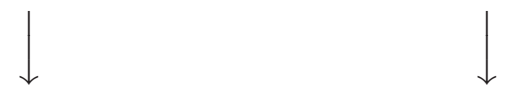


- where the horizontal arrows are embeddings that arise tautologically from the constructions of (iii) and (iv) [cf. [IUTchII], Remark 4.8.1, (i)]; the vertical arrows are isomorphisms; the left-hand vertical arrow arises from the second isomorphism that appears in the final display of (iv); the right-hand vertical arrow is the product of the realifications of copies of the inverse of the second isomorphism that appears in the final display of (ii). In particular, by applying the definition of $\left({ }^{\dagger} \mathcal{F}_{\mathfrak{m o d}}^{\odot}\right)_{j}-$ i.e., in terms of local fractional ideals [cf. (ii)] — together with the products of realification functors

$$
\prod_{j \in \mathbb{F}_{l}^{*}}\left({ }^{\dagger} \mathcal{F}_{\mathfrak{m o d}}^{\odot}\right)_{j} \rightarrow \prod_{j \in \mathbb{F}_{l}^{*}}\left({ }^{\dagger} \mathcal{F}_{\mathfrak{m o d}}^{\odot \mathbb{R}}\right)_{j}
$$

[cf. [FrdI], Proposition 5.3], one obtains an algorithm for constructing, in a fash-

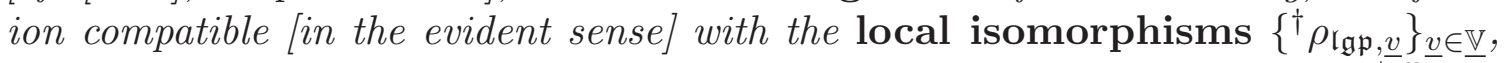

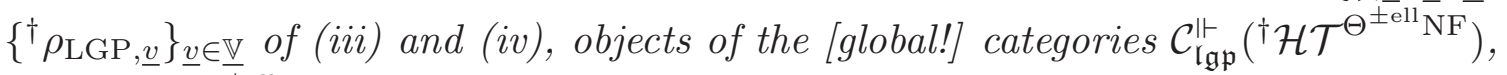
$\mathcal{C}_{\mathrm{LGP}}^{\Vdash}\left({ }^{\dagger} \mathcal{H} \mathcal{T}^{\Theta^{\mp e l l} \mathrm{NF}}\right)$ from the local fractional ideals generated by elements of the monoids [cf. (iv); Proposition 3.4, (ii)]

$$
\Psi_{\mathcal{F}_{\mathfrak{l g} \mathfrak{p}}}\left({ }^{\dagger} \mathcal{H} \mathcal{T}^{\Theta^{ \pm \mathrm{ell}} \mathrm{NF}}\right)_{\underline{v}}
$$

for $\underline{v} \in \underline{\mathbb{V}}^{\text {bad }}$.

Proof. The various assertions of Proposition 3.7 follow immediately from the definitions and the references quoted in the statements of these assertions.

Remark 3.7.1. By analogy to the notation introduced in Proposition 3.7, (i), (ii), we shall write

$$
\left({ }^{\dagger} \mathbb{M}_{\mathrm{ORB}}^{\odot}\right)_{\alpha}=\left({ }^{\dagger} \mathbb{M}_{\mathfrak{o r b}}^{\odot}\right)_{\alpha} \subseteq\left({ }^{\dagger} \overline{\mathbb{M}}_{\mathrm{ORB}}^{\odot}\right)_{\alpha}=\left({ }^{\dagger} \overline{\mathbb{M}}_{\mathfrak{o r b}}^{\odot}\right)_{\alpha}
$$

for the respective images of the maps $\left({ }^{\dagger} \mathbb{M}_{\text {orb }}^{\odot}\right)_{\alpha} \rightarrow \underline{\mathfrak{l o g}_{\text {orb }}}\left({ }^{\alpha} \mathcal{F}_{\mathbb{V}_{\mathbb{Q}}}\right), \quad\left({ }^{\dagger} \overline{\mathbb{M}}_{\text {orb }}^{\odot}\right)_{\alpha} \rightarrow$ $\underline{\log }_{\text {orb }}\left({ }^{\alpha} \mathcal{F}_{\mathbb{V}_{\mathbb{Q}}}\right)$ defined in Remark 3.3.4.

\section{Definition 3.8.}

(i) In the situation of Proposition 3.7, (iv), (v), write $\Psi_{\mathcal{F}_{\mathfrak{l g} \mathfrak{p}}}^{\perp}(-)_{\underline{v}} \stackrel{\text { def }}{=} \Psi_{\mathcal{F}_{\mathrm{LGP}}}^{\perp}(-)_{\underline{v}}$, for $\underline{v} \in \underline{\mathbb{V}}^{\text {bad }}[$ cf. the notation of Proposition 3.5, (ii), (c)]. Then we shall refer to the object of

$$
\prod_{j \in \mathbb{F}_{l}^{*}}\left({ }^{\dagger} \mathcal{F}_{\mathfrak{m} \mathfrak{o d}}^{\odot}\right)_{j}
$$

— as well as its image in $\left.{ }^{\dagger} \mathcal{C}_{\text {LGP }}^{\Vdash}=\mathcal{C}_{\text {LGP }}^{\Vdash}{ }^{\dagger} \mathcal{H} \mathcal{T}^{\Theta^{ \pm e l l} \mathrm{NF}}\right)$ or ${ }^{\dagger} \mathcal{C}_{\mathfrak{l g p}}^{\Vdash}=\mathcal{C}_{\mathfrak{l g p}}^{\Vdash}\left({ }^{\dagger} \mathcal{H} \mathcal{T}^{\Theta^{ \pm e l l} \mathrm{NF}}\right)$ [cf. Proposition 3.7, (iii), (iv), (v)] — determined by any collection, indexed by $\underline{v} \in \underline{\mathbb{V}}^{\text {bad }}$, of generators up to torsion of the monoids $\Psi_{\mathcal{F}_{\mathfrak{l g} \mathfrak{p}}}^{\perp}\left({ }^{\dagger} \mathcal{H} \mathcal{T}^{\Theta^{ \pm \text {ell }} \mathrm{NF}}\right)_{\underline{v}}$ as a $\Theta$-pilot object. We shall refer to the object of the [global realified] Frobenioid

$$
{ }^{\dagger} \mathcal{C}_{\triangle}^{\Vdash}
$$


of [IUTchII], Corollary 4.10, (i), determined by any collection, indexed by $\underline{v} \in \underline{\mathbb{V}}^{\text {bad }}$, of generators up to torsion of the splitting monoid associated to the split Frobenioid $\left({ }^{\dagger} \mathfrak{F}_{\triangle}^{\vdash}\right)_{\underline{v}}$ [i.e., the data indexed by $\underline{v}$ of the $\mathcal{F}^{\vdash}$-prime-strip ${ }^{\dagger} \mathfrak{F}_{\triangle}^{\vdash}$ of [IUTchII], Corollary $4.10, \overline{\mathrm{i}})$ ] — that is to say, at a more concrete level, determined by the " $\underline{\underline{q}} \underline{\underline{v}}$ ", for $\underline{v} \in \underline{\mathbb{V}}^{\text {bad }}[$ cf. the notation of [IUTchI], Example 3.2, (iv)] - as a $\boldsymbol{q}$-pilot object.

(ii) Let

$$
\ddagger \mathcal{H} \mathcal{T}^{\Theta^{ \pm \mathrm{ell}} \mathrm{NF}} \stackrel{\text { log }}{\longrightarrow} \dagger \mathcal{H} \mathcal{T}^{\Theta^{ \pm \mathrm{ell}} \mathrm{NF}}
$$

be a log-link of $\Theta^{ \pm \text {ell }}$ NF-Hodge theaters and

$$
{ }^{*} \mathcal{H} \mathcal{T}^{\Theta}{ }^{ \pm \mathrm{ell}} \mathrm{NF}
$$

a $\Theta^{ \pm \text {ell }} N F$-Hodge theater [all relative to the given initial $\Theta$-data]. Recall the $\mathcal{F}^{\Vdash}$ prime-strip

$$
* \mathfrak{F}_{\triangle}^{\Vdash}
$$

constructed from ${ }^{*} \mathcal{H} \mathcal{T}^{\Theta^{ \pm e l l} \mathrm{NF}}$ in [IUTchII], Corollary 4.10, (i). Following the notational conventions of [IUTchII], Corollary 4.10, (iii), let us write $\mathfrak{F}_{\triangle}$

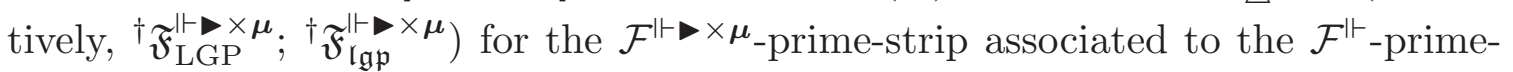
strip ${ }^{*} \mathfrak{F}_{\triangle}\left(\right.$ respectively, ${ }^{\dagger} \mathfrak{F}_{\mathrm{LGP}}^{\Vdash} ;{ }^{\dagger} \mathfrak{F}_{\mathfrak{l g p}}^{\Vdash}$ ) [cf. Proposition 3.7, (iii), (iv); [IUTchII], Definition 4.9, (viii); the functorial algorithm described in [IUTchII], Definition 4.9, (vi)]. Then — in the style of [IUTchII], Corollary 4.10, (iii) — we shall refer

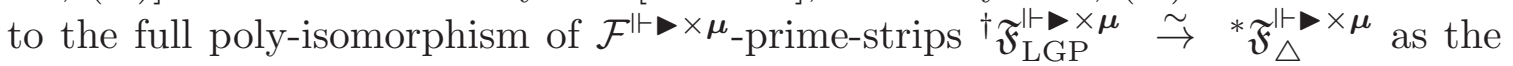
$\Theta_{\mathrm{LGP}}^{\times \mu}-$ link

$$
{ }^{\dagger} \mathcal{H} \mathcal{T}^{\Theta^{ \pm e l l} \mathrm{NF}} \stackrel{\Theta_{\mathrm{LGP}}^{\times \mu}}{\longrightarrow} * \mathcal{H} \mathcal{T}^{\Theta^{ \pm \mathrm{ell}} \mathrm{NF}}
$$

from ${ }^{\dagger} \mathcal{H} \mathcal{T}^{\Theta^{ \pm e l l}} \mathrm{NF}$ to $\ddagger \mathcal{H} \mathcal{T}^{\Theta^{ \pm e l l} \mathrm{NF}}$, relative to the log-link $\ddagger \mathcal{H} \mathcal{T}^{\Theta^{ \pm \mathrm{ell}} \mathrm{NF}} \stackrel{\text { log }}{\longrightarrow}{ }^{\dagger} \mathcal{H} \mathcal{T}^{\Theta^{ \pm e l l} \mathrm{NF}}$,

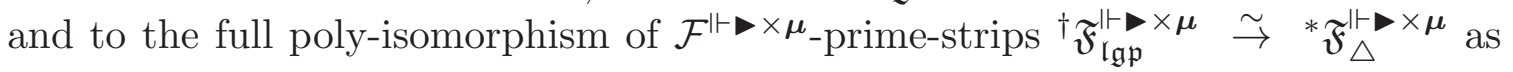
the $\Theta_{\mathfrak{l g p}}^{\times \mu}$-link

$$
\dagger \mathcal{H} \mathcal{T}^{\Theta^{ \pm e l l}} \mathrm{NF} \stackrel{\Theta_{\mathfrak{l g p}}^{\times \mu}}{\longrightarrow} * \mathcal{H} \mathcal{T}^{\Theta^{ \pm e l l} \mathrm{NF}}
$$

from ${ }^{\dagger} \mathcal{H} \mathcal{T}^{\Theta^{ \pm e l l}} \mathrm{NF}$ to ${ }^{\ddagger} \mathcal{H} \mathcal{T}^{\Theta^{ \pm e l l}} \mathrm{NF}$, relative to the log-link $\mathcal{H} \mathcal{T}^{\Theta^{ \pm e l l} \mathrm{NF}} \stackrel{\text { log }}{\longrightarrow} \mathcal{H} \mathcal{T}^{\Theta^{ \pm e l l} \mathrm{NF}}$.

(iii) Let $\left\{{ }^{n, m} \mathcal{H} \mathcal{T}^{\Theta^{ \pm e l l} \mathrm{NF}}\right\}_{n, m \in \mathbb{Z}}$ be a collection of distinct $\Theta^{ \pm \mathrm{ell}} N F$-Hodge theaters [relative to the given initial $\Theta$-data] indexed by pairs of integers. Then we shall refer to the first (respectively, second) diagram

$$
\begin{aligned}
& \begin{array}{ll}
\vdots & \vdots \\
\uparrow \mathfrak{l o g} & \uparrow \mathfrak{l o g}
\end{array}
\end{aligned}
$$

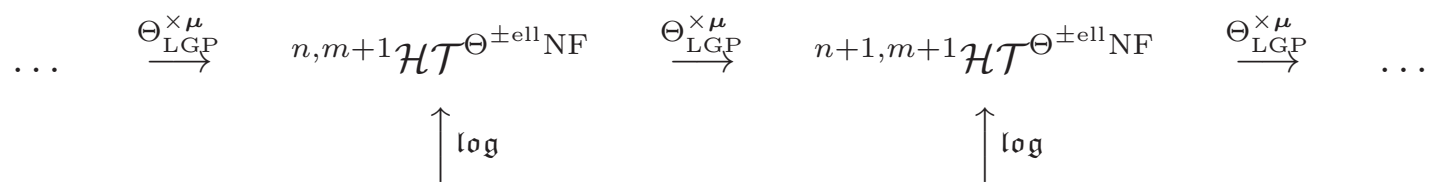

$$
\begin{aligned}
& \ldots \quad \stackrel{\Theta_{\text {LGP }}^{\times \mu}}{\longrightarrow} \quad n, m \mathcal{H} \mathcal{T}^{\Theta^{ \pm e l l} N F} \quad \stackrel{\Theta_{\text {LGP }}^{\times \mu}}{\longrightarrow} \quad n+1, m \mathcal{H} \mathcal{T}^{\Theta^{ \pm e l l} N F} \quad \stackrel{\Theta_{\text {LGP }}^{\times \mu}}{\longrightarrow} \quad \ldots \\
& \uparrow \mathfrak{l o g}
\end{aligned}
$$




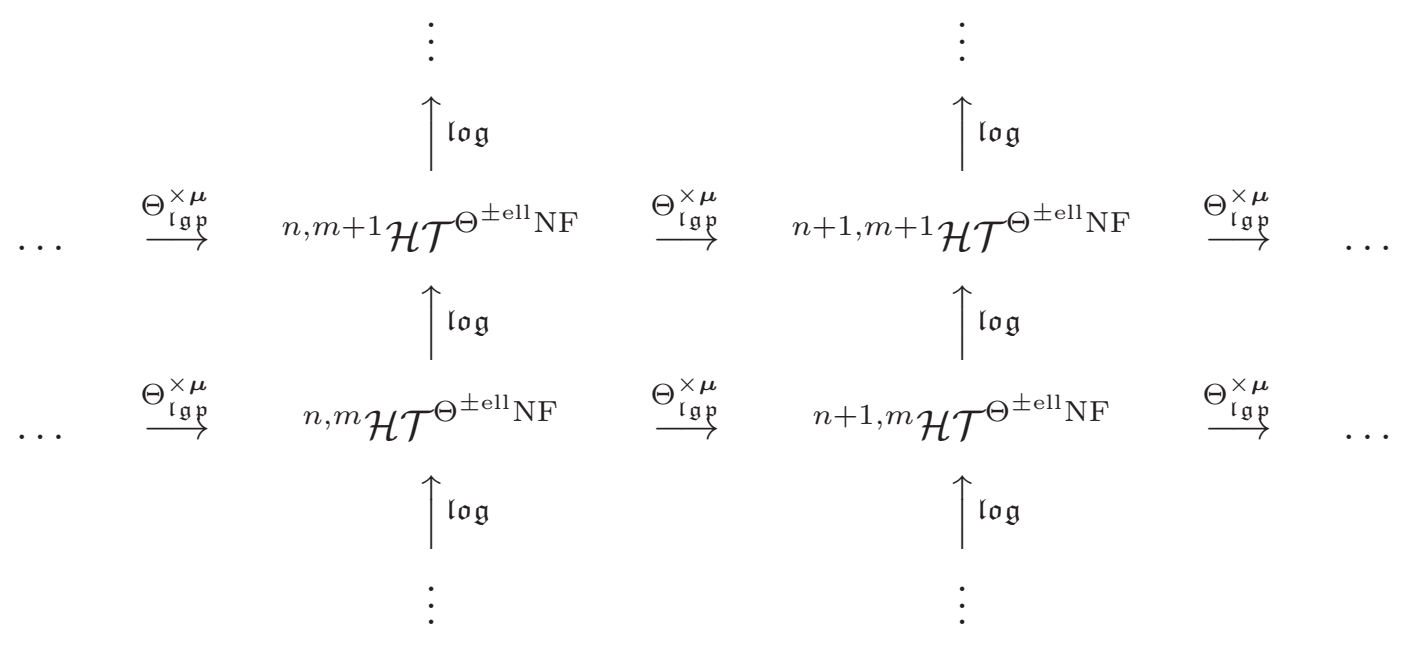

- where the vertical arrows are the full log-links, and the horizontal arrow of the first (respectively, second) diagram from ${ }^{n, m} \mathcal{H} \mathcal{T}^{\Theta^{ \pm \text {ell }} \mathrm{NF}}$ to ${ }^{n+1, m} \mathcal{H} \mathcal{T}^{\Theta^{ \pm e l l}} \mathrm{NF}$ is the $\Theta_{\text {LGP }^{-}}^{\times \boldsymbol{\mu}}$ (respectively, $\Theta_{\mathfrak{l g p}^{-}}^{\times \boldsymbol{\mu}}$ ) link from ${ }^{n, m} \mathcal{H} \mathcal{T}^{\Theta^{ \pm e l l} \mathrm{NF}}$ to ${ }^{n+1, m} \mathcal{H T}^{\Theta^{ \pm \mathrm{ell}} \mathrm{NF}}$, relative to the full log-link ${ }^{n, m-1} \mathcal{H} \mathcal{T}^{\Theta^{ \pm \text {ell }} \mathrm{NF}} \stackrel{\text { log }}{\longrightarrow} n, m \mathcal{H} \mathcal{T}^{\Theta^{ \pm \text {ell }} \mathrm{NF}}$ [cf. (ii)] — as the [LGPGaussian] (respectively, [lgh-Gaussian]) log-theta-lattice. Thus, [cf. Definition 1.4] either of these diagrams may be represented symbolically by an oriented graph

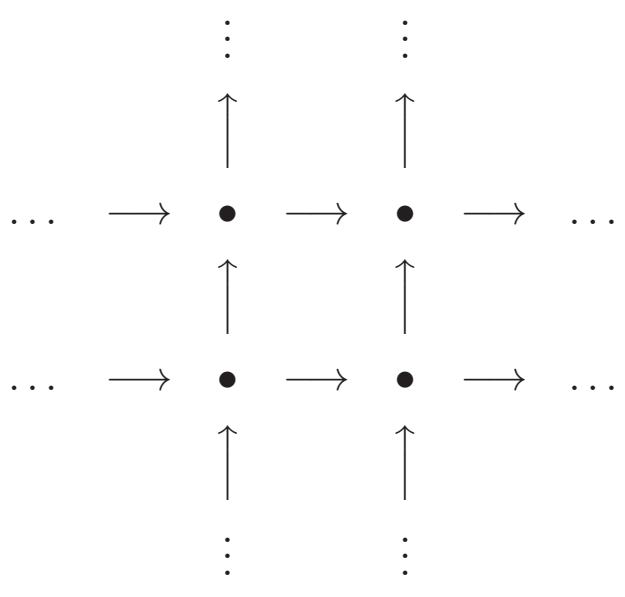

— where the "•'s" correspond to the "n,m $\mathcal{H} \mathcal{T}^{\Theta^{ \pm \text {ell }} \mathrm{NF}}$ ".

Remark 3.8.1. The LGP-Gaussian and $\mathfrak{l g p}$-Gaussian log-theta-lattices are, of course, closely related, but, in the theory to be developed below, we shall mainly be interested in the LGP-Gaussian log-theta-lattice [for reasons to be explained in Remark 3.10.2, (ii), below]. On the other hand, our computation of the $\Theta_{\mathrm{LGP}}^{\times \mu}$-link will involve the $\Theta_{\mathfrak{l g p}}^{\times \boldsymbol{\mu}}$-link, as well as related $\Theta$-pilot objects, in an essential way. Here, we note, for future reference, that both the $\Theta_{\mathrm{LGP}^{-}}^{\times \boldsymbol{\mu}}$ and the $\Theta_{\mathfrak{l g \mathfrak { p }}}^{\times \boldsymbol{\mu}}$-link map $\Theta$-pilot objects to $q$-pilot objects.

Remark 3.8.2. One verifies immediately that the main results obtained so far concerning Gaussian log-theta-lattices — namely, Theorem 1.5, Proposition 2.1, Corollary 2.3, and Proposition 3.5 - generalize immediately [indeed, "formally"] 
to the case of LGP- or lgp-Gaussian log-theta-lattices. Indeed, the substantive content of these results concerns portions of the log-theta-lattices involved that are substantively unaffected by the transition from "Gaussian" to "LGP- or lapGaussian".

Remark 3.8.3. In the definition of the various horizontal arrows of the logtheta-lattices discussed in Definition 3.8, (iii), it may appear to the reader, at first glance, that, instead of working with $\mathcal{F}^{\Vdash}-\times \boldsymbol{\mu}$-prime-strips, it might in fact be sufficient to replace the unit [i.e., $\mathcal{F}^{\vdash \times \boldsymbol{\mu}}$-prime-strip] portions of these primestrips by the associated log-shells [cf. Proposition 1.2, (vi), (vii)], on which, at nonarchimedean $\underline{v} \in \mathbb{V}$, the associated local Galois groups act trivially. In fact, however, this is not the case. That is to say, the nontrivial Galois action on the local unit portions of the $\mathcal{F}^{\Vdash \triangleright \times \boldsymbol{\mu}}$-prime-strips involved is necessary in order to consider the Kummer theory [cf. Proposition 3.5, (i), (ii), as well as Proposition 3.10, (i), (iii); Remark 3.10.1, (ii), below] of the various local and global objects for which the log-shells serve as "multiradial containers" [cf. the discussion of Remark 1.5.2]. Here, we recall that this Kummer theory plays a crucial role in the theory of the present series of papers in relating corresponding Frobenius-like and étale-like objects [cf. the discussion of Remark 1.5.4, (i)].

\section{Proposition 3.9. (Log-volume for Packets and Processions)}

(i) (Local Holomorphic Packets) In the situation of Proposition 3.2, (i), (ii): Suppose that $\underline{\mathbb{V}} \ni \underline{v} \mid v_{\mathbb{Q}} \in \mathbb{V}_{\mathbb{Q}}^{\text {non }}, \alpha \in A$. Then the $p_{v_{\mathbb{Q}}}$-adic log-volume on each of the direct summand $p_{v_{\mathbb{Q}}}$ adic fields of $\mathcal{I}^{\mathbb{Q}}\left({ }^{\alpha} \mathcal{F}_{v_{\mathbb{Q}}}\right), \mathcal{I}^{\mathbb{Q}}\left({ }^{A} \mathcal{F}_{v_{\mathbb{Q}}}\right)$, and $\mathcal{I}^{\mathbb{Q}}\left({ }^{A, \alpha} \mathcal{F}_{\underline{v}}\right)-c f$. the direct sum decompositions of Proposition 3.1, (i), together with the discussion of normalized weights in Remark 3.1.1, (ii) - determines [cf. [AbsTopIII], Proposition 5.7, (i)] log-volumes

$$
\begin{gathered}
\mu_{\alpha, v_{\mathbb{Q}}}^{\log }: \mathfrak{M}\left(\mathcal{I}^{\mathbb{Q}}\left({ }^{\alpha} \mathcal{F}_{v_{\mathbb{Q}}}\right)\right) \rightarrow \mathbb{R} ; \quad \mu_{A, v_{\mathbb{Q}}}^{\log }: \mathfrak{M}\left(\mathcal{I}^{\mathbb{Q}}\left({ }^{A} \mathcal{F}_{v_{\mathbb{Q}}}\right)\right) \rightarrow \mathbb{R} \\
\mu_{A, \alpha, \underline{v}}^{\log }: \mathfrak{M}\left(\mathcal{I}^{\mathbb{Q}}\left({ }^{A, \alpha} \mathcal{F}_{\underline{v}}\right)\right) \rightarrow \mathbb{R}
\end{gathered}
$$

- where we write "M(-)" for the set of compact open subsets of "(-)" — such that the log-volume of each of the "local holomorphic" integral structures of Proposition 3.1, (ii) - i.e., the elements

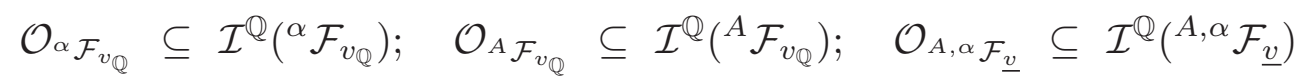

of "MM(-)" given by the integral structures discussed in Proposition 3.1, (ii), on each of the direct summand $p_{v_{\mathbb{Q}}}$-adic fields - is equal to zero. Here, we assume that these log-volumes are normalized so that multiplication of an element of "MM(-)" by $p_{\underline{v}}$ corresponds to adding the quantity $-\log \left(p_{\underline{v}}\right) \in \mathbb{R}$; we shall refer to this normalization as the packet-normalization. Suppose that $\underline{\mathbb{V}} \ni \underline{v} \mid v_{\mathbb{Q}} \in \mathbb{V}_{\mathbb{Q}}^{\text {arc }}, \alpha \in A$. Then the sum of the radial log-volumes on each of the direct summand complex archimedean fields of $\mathcal{I}^{\mathbb{Q}}\left(\alpha \mathcal{F}_{v_{\mathbb{Q}}}\right), \mathcal{I}^{\mathbb{Q}}\left({ }^{A} \mathcal{F}_{v_{\mathbb{Q}}}\right)$, and $\mathcal{I}^{\mathbb{Q}}\left({ }^{A, \alpha} \mathcal{F}_{\underline{v}}\right)-c f$. the direct sum decompositions of Proposition 3.1, (i), together with the discussion of normalized 
weights in Remark 3.1.1, (ii) — determines [cf. [AbsTopIII], Proposition 5.7, (ii)] log-volumes

$$
\begin{gathered}
\mu_{\alpha, v_{\mathbb{Q}}}^{\log }: \mathfrak{M}\left(\mathcal{I}^{\mathbb{Q}}\left({ }^{\alpha} \mathcal{F}_{v_{\mathbb{Q}}}\right)\right) \rightarrow \mathbb{R} ; \quad \mu_{A, v_{\mathbb{Q}}}^{\log }: \mathfrak{M}\left(\mathcal{I}^{\mathbb{Q}}\left({ }^{A} \mathcal{F}_{v_{\mathbb{Q}}}\right)\right) \rightarrow \mathbb{R} \\
\mu_{A, \alpha, \underline{v}}^{\log }: \mathfrak{M}\left(\mathcal{I}^{\mathbb{Q}}\left({ }^{A, \alpha} \mathcal{F}_{\underline{v}}\right)\right) \rightarrow \mathbb{R}
\end{gathered}
$$

- where we write "M(-)" for the set of compact closures of open subsets of "(-)" - such that the log-volume of each of the "local holomorphic" integral structures of Proposition 3.1, (ii) - i.e., the elements

$$
\mathcal{O}_{\alpha \mathcal{F}_{v_{\mathbb{Q}}}} \subseteq \mathcal{I}^{\mathbb{Q}}\left({ }^{\alpha} \mathcal{F}_{v_{\mathbb{Q}}}\right) ; \quad \mathcal{O}_{A \mathcal{F}_{v_{\mathbb{Q}}}} \subseteq \mathcal{I}^{\mathbb{Q}}\left({ }^{A} \mathcal{F}_{v_{\mathbb{Q}}}\right) ; \quad \mathcal{O}_{A, \alpha} \mathcal{F}_{\underline{v}} \subseteq \mathcal{I}^{\mathbb{Q}}\left({ }^{A, \alpha} \mathcal{F}_{\underline{v}}\right)
$$

of "MM(-)" given by the products of the integral structures discussed in Proposition 3.1, (ii), on each of the direct summand complex archimedean fields - is equal to zero. Here, we assume that these log-volumes are normalized so that multiplication of an element of " $\mathfrak{M}(-)$ " by $e=2.71828 \ldots$ corresponds to adding the quantity $1=$ $\log (e) \in \mathbb{R}$; we shall refer to this normalization as the packet-normalization. In both the nonarchimedean and archimedean cases, " $\mu_{A, v_{\mathbb{Q}}}^{\log }$ " is invariant with respect to permutations of A. Finally, when working with collections of capsules in a procession, as in Proposition 3.4, (ii), we obtain, in both the nonarchimedean and archimedean cases, log-volumes on the products of the "MM(-)" associated to the various capsules under consideration, which we normalize by taking the average, over the various capsules under consideration; we shall refer to this normalization as the procession-normalization [cf. Remark 3.9.3 below].

(ii) (Mono-analytic Compatibility) In the situation of Proposition 3.2, (i), (ii): Suppose that $\underline{\mathbb{V}} \ni \underline{v} \mid v_{\mathbb{Q}} \in \mathbb{V} \mathbb{Q}$. Then by applying the $p_{v_{\mathbb{Q}}}$-adic log-volume, when $v_{\mathbb{Q}} \in \mathbb{V}_{\mathbb{Q}}^{\text {non }}$, or the radial log-volume, when $v_{\mathbb{Q}} \in \mathbb{V}_{\mathbb{Q}}^{\text {arc }}$, on the mono-analytic log-shells “ $\mathcal{I}_{\dagger} \mathcal{D}_{\underline{v}}^{+}$" of Proposition 1.2, (vi), (vii), (viii), and adjusting appropriately [cf. Remark 3.9.1 below for more details] to account for the discrepancy between the "local holomorphic" integral structures of Proposition 3.1, (ii), and the "mono-analytic" integral structures of Proposition 3.2, (ii), one obtains [by a slight abuse of notation] log-volumes

$$
\begin{gathered}
\mu_{\alpha, v_{\mathbb{Q}}}^{\log }: \mathfrak{M}\left(\mathcal{I}^{\mathbb{Q}}\left({ }^{\alpha} \mathcal{D}_{v_{\mathbb{Q}}}^{\vdash}\right)\right) \rightarrow \mathbb{R} ; \quad \mu_{A, v_{\mathbb{Q}}}^{\log }: \mathfrak{M}\left(\mathcal{I}^{\mathbb{Q}}\left({ }^{A} \mathcal{D}_{v_{\mathbb{Q}}}^{\vdash}\right)\right) \rightarrow \mathbb{R} \\
\mu_{A, \alpha, \underline{v}}^{\log }: \mathfrak{M}\left(\mathcal{I}^{\mathbb{Q}}\left({ }^{A, \alpha} \mathcal{D}_{\underline{v}}^{\vdash}\right)\right) \rightarrow \mathbb{R}
\end{gathered}
$$

- where "M(-)" is as in (i) above - which are compatible with the log-volumes obtained in (i), relative to the natural poly-isomorphisms of Proposition 3.2, (i). In particular, these log-volumes may be constructed via a functorial algorithm from the $\mathcal{D}^{\vdash}$-prime-strips under consideration. If one considers the monoanalyticization [cf. [IUTchI], Proposition 6.9, (ii)] of a holomorphic procession as in Proposition 3.4, (ii), then taking the average, as in (i) above, of the packetnormalized log-volumes of the above display gives rise to procession-normalized log-volumes, which are compatible, relative to the natural poly-isomorphisms of Proposition 3.2, (i), with the procession-normalized log-volumes of (i). Finally, by replacing " $\mathcal{D}^{\vdash}$ " by " $\mathcal{F}^{\vdash \times \boldsymbol{\mu} "}$ " [cf. also the discussion of Proposition 1.2, (vi), 
(vii), (viii)], one obtains a similar theory of log-volumes for the various objects associated to the mono-analytic log-shells " $\mathcal{I}_{+} \mathcal{F}_{\underline{v}}^{\vdash \times \mu}$ ", which is compatible with the theory obtained for " $\mathcal{D}^{\vdash}$ " relative to the various natural poly-isomorphisms of Proposition 3.2, (i). Write

(iii) (Global Compatibility) In the situation of Proposition 3.7, (i), (ii):

$$
\mathcal{I}^{\mathbb{Q}}\left({ }^{A} \mathcal{F}_{\mathbb{V}_{\mathbb{Q}}}\right) \stackrel{\text { def }}{=} \prod_{v_{\mathbb{Q}} \in \mathbb{V}_{\mathbb{Q}}} \mathcal{I}^{\mathbb{Q}}\left({ }^{A} \mathcal{F}_{v_{\mathbb{Q}}}\right) \subseteq \underline{\mathfrak{l o g}}\left({ }^{A} \mathcal{F}_{\mathbb{V}_{\mathbb{Q}}}\right)=\prod_{v_{\mathbb{Q}} \in \mathbb{V}_{\mathbb{Q}}} \underline{\mathfrak{l o g}}\left({ }^{A} \mathcal{F}_{v_{\mathbb{Q}}}\right)
$$

and

$$
\mathfrak{M}\left(\mathcal{I}^{\mathbb{Q}}\left({ }^{A} \mathcal{F}_{\mathbb{V}_{\mathbb{Q}}}\right)\right) \subseteq \prod_{v_{\mathbb{Q}} \in \mathbb{V}_{\mathbb{Q}}} \mathfrak{M}\left(\mathcal{I}^{\mathbb{Q}}\left({ }^{A} \mathcal{F}_{v_{\mathbb{Q}}}\right)\right)
$$

for the subset of elements whose components, indexed by $v_{\mathbb{Q}} \in \mathbb{V}_{\mathbb{Q}}$, have zero logvolume [cf. (i)] for all but finitely many $v_{\mathbb{Q}} \in \mathbb{V}_{\mathbb{Q}}$. Then, by adding the log-volumes of (i) [all but finitely many of which are zero!] at the various $v_{\mathbb{Q}} \in \mathbb{V}_{\mathbb{Q}}$, one obtains a global log-volume

$$
\mu_{A, \mathbb{V}_{\mathbb{Q}}}^{\log }: \mathfrak{M}\left(\mathcal{I}^{\mathbb{Q}}\left({ }^{A} \mathcal{F}_{\mathbb{V}_{\mathbb{Q}}}\right)\right) \rightarrow \mathbb{R}
$$

which is invariant with respect to multiplication by elements of

$$
\left({ }^{\dagger} \overline{\mathrm{M}}_{\mathrm{mod}}^{\odot}\right)_{\alpha}=\left({ }^{\dagger} \overline{\mathrm{M}}_{\mathrm{MOD}}^{\odot}\right)_{\alpha} \subseteq \mathcal{I}^{\mathbb{Q}}\left({ }^{A} \mathcal{F}_{\mathbb{V}_{\mathbb{Q}}}\right)
$$

as well as with respect to permutations of $A$, and, moreover, satisfies the following property concerning [the elements of "MM( $(-)$ " determined by] objects " $\mathcal{J}=$ $\left\{J_{\underline{v}}\right\}_{\underline{v} \in \underline{\mathbb{V}}}$ " of $\left({ }^{\dagger} \mathcal{F}_{\mathfrak{m o d}}^{\odot}\right)_{\alpha}$ [cf. Example 3.6, (ii); Proposition 3.7, (ii)]: the global log-volume $\mu_{A, \mathbb{V}_{\mathbb{Q}}}^{\log }(\mathcal{J})$ is equal to the degree of the arithmetic line bundle determined by $\mathcal{J}$ [cf. the discussion of Example 3.6, (ii); the natural isomorphism $\left({ }^{\dagger} \mathcal{F}_{\mathfrak{m o d}}^{\odot}\right)_{\alpha} \stackrel{\sim}{\rightarrow}\left({ }^{\dagger} \mathcal{F}_{\bmod }^{\odot}\right)_{\alpha}$ of Proposition 3.7, (ii)], relative to a suitable normalization.

(iv) (log-link Compatibility) Let $\left\{^{n, m} \mathcal{H} \mathcal{T}^{\Theta^{ \pm e l l} \mathrm{NF}}\right\}_{n, m \in \mathbb{Z}}$ be a collection of distinct $\Theta^{ \pm e l l} \mathbf{N F}$-Hodge theaters [relative to the given initial $\Theta$-data] - which we think of as arising from an LGP-Gaussian log-theta-lattice [cf. Definition 3.8, (iii)]. Then [cf. also the discussion of Remark 3.9.4 below]:

(a) For $n, m \in \mathbb{Z}$, the log-volumes constructed in (i), (ii), (iii) above determine log-volumes on the various " $\mathcal{I} \mathbb{Q}((-))$ " that appear in the construction of the local/global LGP-/lgp-monoids/Frobenioids that appear in the $\mathcal{F}^{\Vdash}$-prime-strips ${ }^{n, m} \mathfrak{F}_{\mathrm{LGP}}^{\Vdash},{ }^{n, m} \mathfrak{F}_{\mathfrak{l g p}}^{\Vdash}$ constructed in Proposition 3.7, (iii), (iv), relative to the log-link ${ }^{n, m-1} \mathcal{H} \mathcal{T}^{\Theta^{ \pm \mathrm{ell}} \mathrm{NF}} \stackrel{\text { log }}{\longrightarrow} n, m \mathcal{H} \mathcal{T}^{\Theta^{ \pm e l l} \mathrm{NF}}$.

(b) At the level of the $\mathbb{Q}$-spans of log-shells " $\mathcal{I}((-))$ " that arise from the various $\mathcal{F}$-prime-strips involved, the log-volumes of $(a)$ indexed by $(n, m)$ are compatible - in the sense discussed in Propositions 1.2, (iii); 1.3, (iii) — with the corresponding log-volumes indexed by $(n, m-1)$, relative to the log-link ${ }^{n, m-1} \mathcal{H} \mathcal{T}^{\Theta^{ \pm \mathrm{ell}} \mathrm{NF}} \stackrel{\mathfrak{l o g}}{\longrightarrow} n, m \mathcal{H} \mathcal{T}^{\Theta^{ \pm \mathrm{ell}} \mathrm{NF}}$. 
Proof. The various assertions of Proposition 3.9 follow immediately from the definitions and the references quoted in the statements of these assertions.

Remark 3.9.1. In the spirit of the explicit descriptions of Remark 3.1.1, (i) [cf. also Remark 1.2.2, (i), (ii)], we make the following observations.

(i) Suppose that $v_{\mathbb{Q}} \in \mathbb{V}_{\mathbb{Q}}^{\text {non }}$. Write $\left\{\underline{v}_{1}, \ldots, \underline{v}_{n}\right\}$ for the [distinct!] elements of $\underline{\mathbb{V}}$ that lie over $v_{\mathbb{Q}}$. For each $i=1, \ldots, n$, set $k_{i} \stackrel{\text { def }}{=} K_{\underline{v}_{i}}$; write $\mathcal{O}_{k_{i}} \subseteq k_{i}$ for the ring of integers of $k_{i}$,

$$
\mathcal{I}_{i} \stackrel{\text { def }}{=}\left(p_{v_{\mathbb{Q}}}^{*}\right)^{-1} \cdot \log _{k_{i}}\left(\mathcal{O}_{k_{i}}^{\times}\right) \subseteq k_{i}
$$

- where $p_{v_{\mathbb{Q}}}^{*}=p_{\underline{v}}$ if $p_{v_{\mathbb{Q}}}$ is odd, $p_{v_{\mathbb{Q}}}^{*}=p_{v_{\mathbb{Q}}}^{2}$ if $p_{v_{\mathbb{Q}}}$ is even - cf. Remark 1.2.2, (i). Then, roughly speaking, in the notation of Proposition 3.9, (i), the mono-analytic integral structures of Proposition 3.2, (ii), in

$$
\mathcal{I}^{\mathbb{Q}}\left({ }^{\alpha} \mathcal{F}_{v_{\mathbb{Q}}}\right) \stackrel{\sim}{\rightarrow} \bigoplus_{i=1}^{n} k_{i} ; \quad \mathcal{I}^{\mathbb{Q}}\left({ }^{A} \mathcal{F}_{v_{\mathbb{Q}}}\right) \stackrel{\sim}{\rightarrow} \bigotimes_{\alpha \in A} \mathcal{I}^{\mathbb{Q}}\left({ }^{\alpha} \mathcal{F}_{v_{\mathbb{Q}}}\right)
$$

are given by

$$
\mathcal{I}\left({ }^{\alpha} \mathcal{F}_{v_{\mathbb{Q}}}\right) \stackrel{\sim}{\rightarrow} \bigoplus_{i=1}^{n} \mathcal{I}_{i} ; \quad \mathcal{I}\left({ }^{A} \mathcal{F}_{v_{\mathbb{Q}}}\right) \stackrel{\sim}{\rightarrow} \bigotimes_{\alpha \in A} \mathcal{I}\left({ }^{\alpha} \mathcal{F}_{v_{\mathbb{Q}}}\right)
$$

while the local holomorphic integral structures

$$
\mathcal{O}_{\alpha} \mathcal{F}_{v_{\mathbb{Q}}} \subseteq \mathcal{I}^{\mathbb{Q}}\left({ }^{\alpha} \mathcal{F}_{v_{\mathbb{Q}}}\right) ; \quad \mathcal{O}^{A} \mathcal{F}_{v_{\mathbb{Q}}} \subseteq \mathcal{I}^{\mathbb{Q}}\left({ }^{A} \mathcal{F}_{v_{\mathbb{Q}}}\right)
$$

of Proposition 3.9, (i), in the topological rings $\mathcal{I}^{\mathbb{Q}}\left({ }^{\alpha} \mathcal{F}_{v_{\mathbb{Q}}}\right), \mathcal{I}^{\mathbb{Q}}\left({ }^{A} \mathcal{F}_{v_{\mathbb{Q}}}\right)$ - both of which are direct sums of finite extensions of $\mathbb{Q}_{p_{v_{\mathbb{Q}}}}$ - are given by the subrings of integers in $\mathcal{I}^{\mathbb{Q}}\left({ }^{\alpha} \mathcal{F}_{v_{\mathbb{Q}}}\right), \mathcal{I}^{\mathbb{Q}}\left({ }^{A} \mathcal{F}_{v_{\mathbb{Q}}}\right)$. Thus, by applying the formula of the final display of [AbsTopIII], Proposition 5.8, (iii), for the log-volume of $\mathcal{I}_{i}$, [one verifies easily that] one may compute the log-volumes

$$
\mu_{\alpha, v_{\mathbb{Q}}}^{\log }\left(\mathcal{I}\left({ }^{\alpha} \mathcal{F}_{v_{\mathbb{Q}}}\right)\right), \quad \mu_{A, v_{\mathbb{Q}}}^{\log }\left(\mathcal{I}\left({ }^{A} \mathcal{F}_{v_{\mathbb{Q}}}\right)\right)
$$

entirely in terms of the given initial $\Theta$-data. We leave the routine details to the reader.

(ii) Suppose that $v_{\mathbb{Q}} \in \mathbb{V}_{\mathbb{Q}}^{\text {arc }}$. Write $\left\{\underline{v}_{1}, \ldots, \underline{v}_{n}\right\}$ for the [distinct!] elements of $\underline{\mathbb{V}}$ that lie over $v_{\mathbb{Q}}$. For each $i=1, \ldots, n$, set $k_{i} \stackrel{\text { def }}{=} K_{\underline{v}_{i}} ;$ write $\mathcal{O}_{k_{i}} \subseteq\left\{\lambda \in k_{i}|| \lambda \mid \leq\right.$ $1\} \subseteq k_{i}$ for the "set of integers" of $k_{i}$,

$$
\mathcal{I}_{i} \stackrel{\text { def }}{=} \pi \cdot \mathcal{O}_{k_{i}} \subseteq k_{i}
$$

- cf. Remark 1.2.2, (ii). Then, roughly speaking, in the notation of Proposition 3.9, (i), the discrepancy between the mono-analytic integral structures of 
Proposition 3.2, (ii), determined by the $\mathcal{I}\left({ }^{\dagger} \mathcal{F}_{\underline{v}_{i}}\right) \stackrel{\sim}{\rightarrow} \mathcal{I}_{i} \subseteq k_{i}$ and the local holomorphic integral structures

$$
\begin{aligned}
& \mathcal{O}_{\alpha \mathcal{F}_{v_{\mathbb{Q}}}} \subseteq \mathcal{I}^{\mathbb{Q}}\left({ }^{\alpha} \mathcal{F}_{v_{\mathbb{Q}}}\right) \stackrel{\sim}{\rightarrow} \bigoplus_{i=1}^{n} k_{i} \\
& \mathcal{O}_{A \mathcal{F}_{v_{\mathbb{Q}}}} \subseteq \mathcal{I}^{\mathbb{Q}}\left({ }^{A} \mathcal{F}_{v_{\mathbb{Q}}}\right) \stackrel{\sim}{\rightarrow} \bigotimes_{\alpha \in A} \mathcal{I}^{\mathbb{Q}}\left({ }^{\alpha} \mathcal{F}_{v_{\mathbb{Q}}}\right)
\end{aligned}
$$

of Proposition 3.9, (i), in the topological rings $\mathcal{I}^{\mathbb{Q}}\left({ }^{\alpha} \mathcal{F}_{v_{\mathbb{Q}}}\right), \mathcal{I}^{\mathbb{Q}}\left({ }^{A} \mathcal{F}_{v_{\mathbb{Q}}}\right)$ - both of which are direct sums of complex archimedean fields - determined by taking the product [relative to this direct sum decomposition] of the respective "subsets of integers" may be computed entirely in terms of the given initial $\Theta$-data, by applying the following two [easily verified] observations:

(a) Equip $\mathbb{C}$ with its standard Hermitian metric, i.e., the metric determined by the complex norm. This metric on $\mathbb{C}$ determines a tensor product metric on $\mathbb{C} \otimes_{\mathbb{R}} \mathbb{C}$, as well as a direct sum metric on $\mathbb{C} \oplus \mathbb{C}$. Then, relative to these metrics, any isomorphism of topological rings [i.e., arising from the Chinese remainder theorem]

$$
\mathbb{C} \otimes_{\mathbb{R}} \mathbb{C} \stackrel{\sim}{\rightarrow} \mathbb{C} \oplus \mathbb{C}
$$

is compatible with these metrics, up a factor of 2 , i.e., the metric on the right-hand side corresponds to 2 times the metric on the left-hand side.

(b) Relative to the notation of (a), the direct sum decomposition $\mathbb{C} \oplus \mathbb{C}$, together with its Hermitian metric, is preserved, relative to the displayed isomorphism of (a), by the operation of conjugation on either of the two copies of "C્C" that appear in $\mathbb{C} \otimes_{\mathbb{R}} \mathbb{C}$, as well as by the operations of multiplying by \pm 1 or $\pm \sqrt{-1}$ via either of the two copies of " $\mathbb{C}$ " that appear in $\mathbb{C} \otimes_{\mathbb{R}} \mathbb{C}$.

We leave the routine details to the reader.

(iii) The computation of the discrepancy between local holomorphic and monoanalytic integral structures will be discussed in more detail in [IUTchIV].

Remark 3.9.2. In the situation of Proposition 3.9, (iii), one may construct ["mono-analytic"] algorithms for recovering the subquotient of the perfection of $\left({ }^{\dagger} \mathbb{M}_{\mathfrak{m o d}}^{\odot}\right)_{\alpha}=\left({ }^{\dagger} \mathbb{M}_{\mathrm{MOD}}^{\odot}\right)_{\alpha}$ associated to $\underline{w} \in \underline{\mathbb{V}}[\mathrm{cf}$. Remark 3.6.1], together with the submonoid of "nonnegative elements" of such a subquotient, by considering the effect of multiplication by elements of $\left({ }^{\dagger} \mathbb{M}_{\mathfrak{m o d}}^{\odot}\right)_{\alpha}=\left({ }^{\dagger} \mathbb{M}_{\mathrm{MOD}}^{\odot}\right)_{\alpha}$ on the log-volumes defined on the various $\mathcal{I}^{\mathbb{Q}}\left(A, \alpha \mathcal{F}_{\underline{v}}\right) \stackrel{\sim}{\rightarrow} \mathcal{I}^{\mathbb{Q}}\left(A, \alpha \mathcal{D}_{\underline{v}}^{\vdash}\right)$ [cf. Proposition 3.9, (ii)].

Remark 3.9.3. With regard to the procession-normalizations discussed in Proposition 3.9, (i), (ii), the reader might wonder the following: Is it possible to work with 
more general weighted averages, i.e., as opposed to just averages, in the usual sense, over the capsules that appear in the procession?

The answer to this question is "no". Indeed, in the situation of Proposition 3.4, (ii), for $j \in\left\{1, \ldots, l^{*}\right\}$, the packet-normalized log-volume corresponding to the capsule with index set $\mathbb{S}_{j+1}^{ \pm}$may be thought of as a log-volume that arises from "any one of the log-shells whose label $\in\{0,1, \ldots, j\}$ ". In particular, if $j^{\prime}, j_{1}, j_{2} \in$ $\{1, \ldots, l *\}$, and $j^{\prime} \leq j_{1}, j_{2}$, then log-volumes corresponding to the same log-shell labeled $j^{\prime}$ might give rise to packet-normalized log-volumes corresponding to either of [the capsules with index sets] $\mathbb{S}_{j_{1}+1}^{ \pm}, \mathbb{S}_{j_{2}+1}^{ \pm}$. That is to say, in order for the resulting notion of a procession-normalized log-volume to be compatible with the appearance of the component labeled $j^{\prime}$ in various distinct capsules of the procession - i.e., compatible with the various inclusion morphisms of the procession! one has no choice but to assign the same weights to [the capsules with index sets] $\mathbb{S}_{j_{1}+1}^{ \pm}, \mathbb{S}_{j_{2}+1}^{ \pm}$

Remark 3.9.4. One way to understand the significance of the log-link compatibility of log-volumes discussed in Proposition 3.9, (iv), is as follows. Suppose that instead of knowing this property, one only knows that

each application of the log-link has the effect of dilating volumes by a factor $\lambda \in \mathbb{R}_{>0}$, i.e., which is not necessarily equal to 1 .

Then in order to compute log-volumes in a fashion that is consistent with the various arrows [i.e., both Kummer isomorphisms and log-links!] of the "systems" constituted by the log-Kummer correspondences discussed in Proposition 3.5, (ii), it would be necessary, whenever $\lambda \neq 1$, to regard the various "log-volumes" computed as only giving rise to well-defined elements [not $\in \mathbb{R}$, but rather]

$$
\in \quad \mathbb{R} / \mathbb{Z} \cdot \log (\lambda) \quad\left(\cong \mathbb{S}^{1}\right)
$$

- a situation which is not acceptable, relative to the goal of obtaining estimates [i.e., as in Corollary 3.12 below] for the various objects for which log-shells serve as "multiradial containers" [cf. the discussion of Remark 1.5.2; the content of Theorem 3.11 below].

Proposition 3.10. (Global Kummer Theory and Non-interference with Local Integers) Let $\left\{{ }^{n, m} \mathcal{H} \mathcal{T}^{\Theta^{ \pm e l l} \mathrm{NF}}\right\}_{n, m \in \mathbb{Z}}$ be a collection of distinct $\Theta^{ \pm \text {ell }} \mathrm{NF}$ Hodge theaters [relative to the given initial $\Theta$-data] - which we think of as arising from an LGP-Gaussian log-theta-lattice [cf. Definition 3.8, (iii); Proposition 3.5; Remark 3.8.2]. For each $n \in \mathbb{Z}$, write

$$
{ }^{n,{ }^{\circ}} \mathcal{H} \mathcal{T}^{\mathcal{D}-\Theta^{ \pm \mathrm{ell}} \mathrm{NF}}
$$

for the $\mathcal{D}-\Theta^{ \pm \text {ell }} N F$-Hodge theater determined, up to isomorphism, by the various ${ }^{n, m} \mathcal{H} \mathcal{T}^{\Theta^{ \pm e l l} \mathrm{NF}}$, where $m \in \mathbb{Z}$, via the vertical coricity of Theorem 1.5, (i) [cf. Remark 3.8.2]. 
(i) (Vertically Coric Global LGP-, lgap-Frobenioids and Associated Kummer Theory) Recall the constructions of various global Frobenioids in Proposition 3.7, (i), (ii), (iii), (iv), in the context of $\mathcal{F}$-prime-strip processions. Then by applying these constructions to the $\mathcal{F}$-prime-strips " $\mathfrak{F}\left(n,{ }^{\circ} \mathfrak{D}_{\succ}\right)_{t}$ " [cf. the notation of Proposition 3.5, (i)] and the various full log-links associated [cf. the discussion of Proposition 1.2, (ix)] to these $\mathcal{F}$-prime-strips - which we consider in a fashion compatible with the $\mathbb{F}_{l}^{\rtimes \pm}$-symmetries involved [cf. Remark 1.3.2; Proposition 3.4, (ii)] - we obtain functorial algorithms in the $\mathcal{D}-\Theta^{ \pm \text {ell }} \mathbf{N F}-$ Hodge theater ${ }^{n, o} \mathcal{H} \mathcal{T}^{\mathcal{D}-\Theta^{ \pm e l l} \mathrm{NF}}$ for constructing [number] fields, monoids, and Frobenioids equipped with natural isomorphisms

$$
\begin{aligned}
& \overline{\mathbb{M}}_{\mathfrak{m} \mathfrak{d} \mathfrak{d}}^{\odot}\left({ }^{n, \circ} \mathcal{H} \mathcal{T}^{\mathcal{D}-\Theta^{ \pm \mathrm{ell}} \mathrm{NF}}\right)_{\alpha}=\overline{\mathbb{M}}_{\mathrm{MOD}}^{\odot}\left({ }^{n, \circ} \mathcal{H} \mathcal{T}^{\mathcal{D}-\Theta^{ \pm \mathrm{ell}} \mathrm{NF}}\right)_{\alpha} \\
& \supseteq \mathbb{M}_{\mathfrak{m o d}}^{\odot}\left({ }^{n, o} \mathcal{H} \mathcal{T}^{\mathcal{D}-\Theta^{ \pm e l l} \mathrm{NF}}\right)_{\alpha}=\mathbb{M}_{\mathrm{MOD}}^{\odot}\left({ }^{n, \circ} \mathcal{H} \mathcal{T}^{\mathcal{D}-\Theta^{ \pm \mathrm{ell}} \mathrm{NF}}\right)_{\alpha} \\
& \overline{\mathbb{M}}_{\text {mod }}^{\odot}\left({ }^{n, o} \mathcal{H} \mathcal{T}^{\mathcal{D}-\Theta^{ \pm e l l} \mathrm{NF}}\right)_{\alpha} \supseteq \mathbb{M}_{\text {mod }}^{\odot}\left({ }^{n, o} \mathcal{H} \mathcal{T}^{\mathcal{D}-\Theta^{ \pm e l l} \mathrm{NF}}\right)_{\alpha} \\
& \mathcal{F}_{\text {mod }}^{\odot}\left({ }^{n, o} \mathcal{H} \mathcal{T}^{\mathcal{D}-\Theta^{ \pm \mathrm{ell}} \mathrm{NF}}\right)_{\alpha} \stackrel{\sim}{\rightarrow} \mathcal{F}_{\mathrm{mod}}^{\odot}\left({ }^{n, o} \mathcal{H} \mathcal{T}^{\mathcal{D}-\Theta^{ \pm \mathrm{ell}} \mathrm{NF}}\right)_{\alpha} \stackrel{\sim}{\rightarrow} \mathcal{F}_{\mathrm{MOD}}^{\odot}\left({ }^{n, o} \mathcal{H} \mathcal{T}^{\mathcal{D}-\Theta^{ \pm \mathrm{ell}} \mathrm{NF}}\right)_{\alpha}
\end{aligned}
$$

[cf. the number fields, monoids, and Frobenioids $" \overline{\mathbb{M}}_{\bmod }^{\odot}\left({ }^{\dagger} \mathcal{D}^{\odot}\right)_{j} \supseteq \mathbb{M}_{\bmod }^{\odot}\left({ }^{\dagger} \mathcal{D}^{\odot}\right)_{j} "$, " $\mathcal{F}_{\text {mod }}^{\odot}\left({ }^{\dagger} \mathcal{D}^{\odot}\right)_{j}$ " of [IUTchII], Corollary 4.7, (ii)] for $\alpha \in A$, where $A$ is a subset of $J$ [cf. Proposition 3.3], as well as $\mathcal{F}^{\Vdash}$-prime-strips equipped with natural isomorphisms

$$
\mathfrak{F}^{\Vdash}\left({ }^{n, \circ} \mathcal{H} \mathcal{T}^{\mathcal{D}-\Theta^{ \pm e l l} \mathrm{NF}}\right)_{\text {gau }} \stackrel{\sim}{\rightarrow} \mathfrak{F}^{\Vdash}\left({ }^{n, o} \mathcal{H} \mathcal{T}^{\mathcal{D}-\Theta^{ \pm e l l} \mathrm{NF}}\right)_{\mathrm{LGP}} \stackrel{\sim}{\rightarrow} \mathfrak{F}^{\Vdash}\left({ }^{n, o} \mathcal{H} \mathcal{T}^{\mathcal{D}-\Theta^{ \pm \mathrm{ell}} \mathrm{NF}}\right)_{\mathfrak{l g p}}
$$

- [all of] which we shall refer to as being "vertically coric". For each $n, m \in \mathbb{Z}$, these functorial algorithms are compatible [in the evident sense] with the ["nonvertically coric"] functorial algorithms of Proposition 3.7, (i), (ii), (iii), (iv) i.e., where [in Proposition 3.7, (iii), (iv)] we take "†" to be " $n, m$ " and "f" to be " $n, m-1 "$ - relative to the Kummer isomorphisms of labeled data

$$
\begin{gathered}
\Psi_{\mathrm{cns}}\left({ }^{n, m^{\prime}} \mathfrak{F}_{\succ}\right)_{t} \stackrel{\sim}{\rightarrow} \quad \Psi_{\mathrm{cns}}\left({ }^{n,{ }^{\circ}} \mathfrak{D}_{\succ}\right)_{t} \\
\left({ }^{n, m^{\prime}} \mathbb{M}_{\mathrm{mod}}^{\odot}\right)_{j} \stackrel{\sim}{\rightarrow} \mathbb{M}_{\text {mod }}^{\odot}\left({ }^{n, \circ} \mathcal{D}^{\odot}\right)_{j} ; \quad\left({ }^{n, m^{\prime}} \overline{\mathbb{M}}_{\text {mod }}^{\odot}\right)_{j} \stackrel{\sim}{\rightarrow} \overline{\mathbb{M}}_{\text {mod }}^{\odot}\left({ }^{n, \circ} \mathcal{D}^{\odot}\right)_{j}
\end{gathered}
$$

[cf. [IUTchII], Corollary 4.6, (iii); [IUTchII], Corollary 4.8, (ii)] and the evident identification, for $m^{\prime}=m, m-1$, of ${ }^{n, m^{\prime}} \mathfrak{F}_{t}$ [i.e., the $\mathcal{F}_{\text {-prime-strip that appears }}$ in the associated $\Theta^{ \pm}$-bridge] with the $\mathcal{F}$-prime-strip associated to $\Psi_{\mathrm{cns}}\left({ }^{n, m^{\prime}} \mathfrak{F}_{\succ}\right)_{t}$ [cf. Proposition 3.5, (i)]. In particular, for each $n, m \in \mathbb{Z}$, we obtain "Kummer isomorphisms" of fields, monoids, Frobenioids, and $\mathcal{F}^{\Vdash}$-prime-strips

$$
\begin{aligned}
& \left({ }^{n, m} \overline{\mathbb{M}}_{\mathfrak{m o d}}^{\odot}\right)_{\alpha} \stackrel{\sim}{\rightarrow} \overline{\mathbb{M}}_{\mathfrak{m o d}}^{\odot}\left({ }^{n, o} \mathcal{H} \mathcal{T}^{\mathcal{D}-\Theta^{ \pm e l 1} \mathrm{NF}}\right)_{\alpha} ; \quad\left({ }^{n, m} \overline{\mathbb{M}}_{\mathrm{MOD}}^{\odot}\right)_{\alpha} \stackrel{\sim}{\rightarrow} \overline{\mathbb{M}}_{\mathrm{MOD}}^{\odot}\left({ }^{n, \circ} \mathcal{H} \mathcal{T}^{\mathcal{D}-\Theta^{ \pm \mathrm{ell}} \mathrm{NF}}\right)_{\alpha} \\
& \left({ }^{n, m_{1}} \mathbb{M}_{\mathfrak{m o d}}^{\odot}\right)_{\alpha} \stackrel{\sim}{\rightarrow} \mathbb{M}_{\mathfrak{m o d}}^{\odot}\left({ }^{n, o} \mathcal{H} \mathcal{T}^{\mathcal{D}-\Theta^{ \pm e l l} \mathrm{NF}}\right)_{\alpha} ; \quad\left({ }^{n, m} \mathbb{M}_{\mathrm{MOD}}^{\odot}\right)_{\alpha} \stackrel{\sim}{\rightarrow} \mathbb{M}_{\mathrm{MOD}}^{\odot}\left({ }^{n, o} \mathcal{H} \mathcal{T}^{\mathcal{D}-\Theta^{ \pm \mathrm{ell}} \mathrm{NF}}\right)_{\alpha} \\
& \left({ }^{n, m} \mathcal{F}_{\mathfrak{m o d}}^{\odot}\right)_{\alpha} \stackrel{\sim}{\rightarrow} \mathcal{F}_{\mathfrak{m o d}}^{\odot}\left({ }^{n, \circ} \mathcal{H} \mathcal{T}^{\mathcal{D}-\Theta^{ \pm \mathrm{ell}} \mathrm{NF}}\right)_{\alpha} ; \quad\left({ }^{n, m} \mathcal{F}_{\mathrm{MOD}}^{\odot}\right)_{\alpha} \stackrel{\sim}{\rightarrow} \mathcal{F}_{\mathrm{MOD}}^{\odot}\left({ }^{n, \circ} \mathcal{H} \mathcal{T}^{\mathcal{D}-\Theta^{ \pm \mathrm{ell}} \mathrm{NF}}\right)_{\alpha} \\
& \left({ }^{n, m} \overline{\mathbb{M}}_{\text {mod }}^{\odot}\right)_{\alpha} \stackrel{\sim}{\rightarrow} \overline{\mathbb{M}}_{\text {mod }}^{\odot}\left({ }^{n, \circ} \mathcal{H} \mathcal{T}^{\mathcal{D}-\Theta^{ \pm e l 1}} \mathrm{NF}\right)_{\alpha} ; \quad\left({ }^{n, m_{1}} \mathbb{M}_{\text {mod }}^{\odot}\right)_{\alpha} \stackrel{\sim}{\rightarrow} \mathbb{M}_{\bmod }^{\odot}\left({ }^{n, \circ} \mathcal{H} \mathcal{T}^{\mathcal{D}-\Theta^{ \pm e l l} \mathrm{NF}}\right)_{\alpha}
\end{aligned}
$$




$$
\begin{aligned}
& \left({ }^{n, m} \mathcal{F}_{\text {mod }}^{\odot}\right)_{\alpha} \stackrel{\sim}{\rightarrow} \mathcal{F}_{\text {mod }}^{\odot}\left({ }^{n, o} \mathcal{H} \mathcal{T}^{\mathcal{D}-\Theta^{ \pm e l l} \mathrm{NF}}\right)_{\alpha} ; \quad{ }^{n, m} \mathfrak{F}_{\text {gau }}^{\Vdash} \stackrel{\sim}{\rightarrow} \mathfrak{F}^{\Vdash \vdash}\left({ }^{n, \circ} \mathcal{H} \mathcal{T}^{\mathcal{D}-\Theta^{ \pm e l l} \mathrm{NF}}\right)_{\text {gau }} \\
& n, m \mathfrak{F}_{\mathrm{LGP}}^{\Vdash} \stackrel{\sim}{\rightarrow} \mathfrak{F}^{\Vdash}\left({ }^{n, o} \mathcal{H} \mathcal{T}^{\mathcal{D}-\Theta^{ \pm \mathrm{ell}} \mathrm{NF}}\right)_{\mathrm{LGP}} ; \quad{ }^{n, m} \mathfrak{F}_{\mathfrak{l g p}}^{\Vdash} \stackrel{\sim}{\rightarrow} \mathfrak{F}^{\Vdash}\left({ }^{n, o} \mathcal{H} \mathcal{T}^{\mathcal{D}-\Theta^{ \pm \text {ell }} \mathrm{NF}}\right)_{\mathfrak{l} \mathfrak{p}}
\end{aligned}
$$

that are compatible with the various equalities, natural inclusions, and natural isomorphisms discussed above.

(ii) (Non-interference with Local Integers) In the notation of Propositions 3.2, (ii); 3.4, (i); 3.7, (i), (ii); 3.9, (iii), we have

$$
\begin{gathered}
\left.\left({ }^{\dagger} \mathbb{M}_{\mathrm{MOD}}^{\odot}\right)_{\alpha} \bigcap \prod_{\underline{v} \in \underline{\mathbb{V}}} \Psi_{\mathfrak{l o g}(A, \alpha} \mathcal{F}_{\underline{v}}\right)=\left({ }^{\dagger} \mathbb{M}_{\mathrm{MOD}}^{\odot \boldsymbol{\mu}}\right)_{\alpha} \\
\left(\subseteq \prod_{\underline{\underline{v}} \in \underline{\mathbb{V}}} \mathcal{I}^{\mathbb{Q}}\left({ }^{A, \alpha} \mathcal{F}_{\underline{v}}\right)=\prod_{v_{\mathbb{Q}} \in \mathbb{V}_{\mathbb{Q}}} \mathcal{I}^{\mathbb{Q}}\left({ }^{A} \mathcal{F}_{v_{\mathbb{Q}}}\right)=\mathcal{I}^{\mathbb{Q}}\left({ }^{A} \mathcal{F}_{\mathbb{V}_{\mathbb{Q}}}\right)\right)
\end{gathered}
$$

- where we write $\left({ }^{\dagger} \mathbb{M}_{\mathrm{MOD}}^{\odot \mu}\right)_{\alpha} \subseteq\left({ }^{\dagger} \mathbb{M}_{\mathrm{MOD}}^{\odot}\right)_{\alpha}$ for the [finite] subgroup of torsion elements, i.e., roots of unity; we identify the product $\prod_{\underline{\mathbb{V}} \ni \underline{v} \mid v_{\mathbb{Q}} \in \mathbb{V}_{\mathbb{Q}}} \mathcal{I}^{\mathbb{Q}}\left({ }^{A, \alpha} \mathcal{F}_{\underline{v}}\right)$ with $\mathcal{I}^{\mathbb{Q}}\left({ }^{A} \mathcal{F}_{v_{\mathbb{Q}}}\right)$. Now let us think of the various groups

$$
\left({ }^{n, m} \mathbb{M}_{\mathrm{MOD}}^{\odot}\right)_{j}
$$

[of nonzero elements of a number field] as acting on various portions of the modules

$$
\mathcal{I}^{\mathbb{Q}}\left({ }^{\mathbb{S}_{j+1}^{ \pm}} \mathcal{F}\left({ }^{n, \circ} \mathfrak{D}_{\succ}\right) \mathbb{V}_{\mathbb{Q}}\right)
$$

[cf. the notation of Proposition 3.5, (ii)] not via a single Kummer isomorphism as in (i), but rather via the totality of the various pre-composites of the relevant Kummer isomorphism with the various iterates of the log-links of the LGP-Gaussian log-theta-lattice — i.e., which is possible, up to [harmless!] "identity indeterminacies" at an adjacent " $m$ ", precisely as a consequence of the equality of the first display of the present (ii) [cf. the discussion of Remark 1.2.3, (ii); the discussion of Definition 1.1, (ii), concerning quotients by $\Psi_{\dagger \mathcal{F}_{v}}^{\boldsymbol{\mu}_{N}}$ at $\underline{v} \in \underline{\mathbb{V}}^{\text {arc }}$; the discussion of Definition 1.1, (iv), at $\left.\underline{v} \in \underline{\mathbb{V}}^{\text {non }}\right]$. Then one obtains a sort of "logKummer correspondence" between the totality, as $m$ ranges over the elements of $\mathbb{Z}$, of the various groups [of nonzero elements of a number field] just discussed [i.e., which are labeled by " $n, m$ "] and their actions on the " $\mathcal{I}$ " labeled by " $n, \circ$ " which is invariant with respect to the translation symmetries [cf. Proposition 1.3, (iv)] of the n-th column of the LGP-Gaussian log-theta-lattice [cf. the discussion of Remark 1.2.2, (iii)].

(iii) (Frobenioid-theoretic log-Kummer Correspondences) The relevant Kummer isomorphisms of (i) induce, via the "log-Kummer correspondence" of (ii) [cf. also Proposition 3.7, (i); Remarks 3.6.1, 3.9.2], isomorphisms of Frobenioids

$$
\begin{aligned}
&\left({ }^{n, m} \mathcal{F}_{\mathrm{MOD}}^{\odot}\right)_{\alpha} \stackrel{\sim}{\rightarrow} \mathcal{F}_{\mathrm{MOD}}^{\odot}\left({ }^{n, \circ} \mathcal{H} \mathcal{T}^{\mathcal{D}-\Theta^{ \pm \mathrm{ell}} \mathrm{NF}}\right)_{\alpha} \\
&\left({ }^{n, m} \mathcal{F}_{\mathrm{MOD}}^{\odot \mathbb{R}}\right)_{\alpha} \stackrel{\sim}{\rightarrow} \mathcal{F}_{\mathrm{MOD}}^{\odot \mathbb{R}}\left({ }^{n, \circ} \mathcal{H} \mathcal{T}^{\mathcal{D}-\Theta^{ \pm e l l} \mathrm{NF}}\right)_{\alpha}
\end{aligned}
$$


that are mutually compatible, as $m$ varies over the elements of $\mathbb{Z}$, with the log-links of the LGP-Gaussian log-theta-lattice. Moreover, these compatible isomorphisms of Frobenioids, together with the relevant Kummer isomorphisms of (i), induce, via the global "log-Kummer correspondence" of (ii) and the splitting monoid portion of the "log-Kummer correspondence" of Proposition 3.5, (ii), isomorphisms of associated $\mathcal{F}^{\Vdash \perp}$-prime-strips [cf. Definition 2.4, (iii)]

$$
{ }_{n, m} \mathfrak{F}_{\mathrm{LGP}}^{\Vdash \perp} \stackrel{\sim}{\rightarrow} \mathfrak{F}^{\Vdash \perp}\left({ }^{n, o} \mathcal{H} \mathcal{T}^{\mathcal{D}-\Theta^{ \pm \text {ell }} \mathrm{NF}}\right)_{\mathrm{LGP}}
$$

that are mutually compatible, as $m$ varies over the elements of $\mathbb{Z}$, with the log-links of the LGP-Gaussian log-theta-lattice.

Proof. The various assertions of Proposition 3.10 follow immediately from the definitions and the references quoted in the statements of these assertions. Here, we observe that the computation of the intersection of the first display of (ii) is an immediate consequence of the well-known fact that the set of nonzero elements of a number field that are integral at all of the places of the number field consists of the set of roots of unity contained in the number field [cf. the discussion of Remark 1.2.3, (ii); [Lang], p. 144, the proof of Theorem 5].

\section{Remark 3.10.1.}

(i) Observe that it follows immediately from the well-known fact from elementary algebraic number theory applied in the proof of Proposition 3.10, (ii), that the first display of Proposition 3.10, (ii), admits a Galois-orbit-theoretic analogue:

$$
\left({ }^{\dagger} \mathbb{M}_{\mathrm{ORB}}^{\odot}\right)_{\alpha} \bigcap \prod_{\underline{v} \in \underline{\mathbb{V}}}\left(\Psi_{\mathfrak{l o g}\left(\alpha \mathcal{F}_{\underline{v}}\right)}\right)_{\text {orb }}=\left({ }^{\dagger} \mathbb{M}_{\mathrm{ORB}}^{\odot \mu}\right)_{\alpha} \quad\left(\subseteq \underline{\log }_{\text {orb }}\left({ }^{\alpha} \mathcal{F}_{\mathbb{V}_{\mathbb{Q}}}\right)\right)
$$

— where we write

$$
\left(\Psi_{\log \left(\alpha \mathcal{F}_{\underline{v}}\right)}\right)_{\text {orb }}
$$

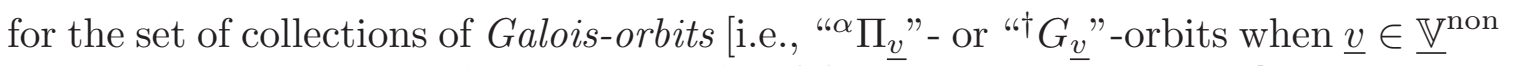
— cf. Propositions 1.2, (vi), and 3.1, (i); $\{1\}$-orbits, when $\left.\underline{v} \in \underline{\mathbb{V}}^{\text {arc }}\right]$ of $\left.\bar{\Psi}_{\mathfrak{l o g}\left({ }^{\alpha}\right.} \mathcal{F}_{\underline{v}}\right)$ [cf. the discussion of Remark 3.3.4] and, in the notation of Remark 3.7.1,

$$
\left({ }^{\dagger} \mathbb{M}_{\mathrm{ORB}}^{\odot \boldsymbol{\mu}}\right)_{\alpha}=\left({ }^{\dagger} \mathbb{M}_{\mathfrak{o r b}}^{\odot \boldsymbol{\mu}}\right)_{\alpha} \subseteq\left({ }^{\dagger} \mathbb{M}_{\mathrm{ORB}}^{\odot}\right)_{\alpha}=\left({ }^{\dagger} \mathbb{M}_{\mathfrak{o r b}}^{\odot}\right)_{\alpha}
$$

for the subset of collections of Galois-orbits all of whose members are roots of unity.

(ii) Write

$$
\left(\left(\Psi_{\mathfrak{l o g}\left(\alpha \mathcal{F}_{\underline{v}}\right)}\right)_{\text {orb }} \supseteq\right) \quad\left(\Psi_{\mathfrak{l o g}\left(\alpha \mathcal{F}_{\underline{v}}\right)}^{\times}\right)_{\text {orb }} \quad \rightarrow \quad\left(\Psi_{\mathfrak{l o g}\left(\alpha \mathcal{F}_{\underline{v}}\right)}^{\times \boldsymbol{\mu}}\right)_{\text {orb }}
$$

for the respective sets of collections of Galois-orbits [i.e., " ${ }^{\alpha} \Pi_{\underline{v}}$ "- or "† $G_{\underline{v}}$ "-orbits when $\underline{v} \in \underline{\mathbb{V}}^{\text {non }}$ - cf. Propositions 1.2 , (vi), and 3.1, (i); $\{1\}$-orbits, when $\left.\underline{\underline{v}} \in \underline{\mathbb{V}}^{\text {arc }}\right]$ of $\Psi_{\mathfrak{l o g}\left({ }^{\alpha} \mathcal{F}_{\underline{v}}\right)}^{\times}, \Psi_{\mathfrak{l o g}\left(\alpha \mathcal{F}_{\underline{v}}\right)}^{\times \boldsymbol{\mu}}[\mathrm{cf}$. the discussion of (i) above] and

$$
\log \left({ }^{\alpha} \mathfrak{F}\right)^{\vdash \times \mu} \stackrel{\text { def }}{=}\left\{\mathfrak{l o g}\left({ }^{\alpha} \mathcal{F}_{\underline{v}}\right)^{\vdash \times \mu}\right\}_{\underline{v} \in \underline{\mathbb{V}}}
$$


for the $\mathcal{F}^{\vdash \times \boldsymbol{\mu}}$-prime-strip determined [cf. [IUTchII], Definition 4.9, (vi), (vii)] by the $\mathcal{F}$-prime-strip $\log \left({ }^{\alpha} \mathfrak{F}\right)$ [cf. Definition 1.1, (iii)]. Then one way to interpret the computation of the intersection of the first display of (i) is as an analogue for " $\mathbb{M}^{\odot}$ " of the multiradiality for mono-theta environments discussed in Theorem 2.2 , (ii); Remark 2.2.1 [cf., especially, Fig. 2.2]; Corollary 2.3, (iv) - i.e., as a sort of multiradiality for number fields to the effect that

the cyclotomic rigidity isomorphism and related Kummer theory of [IUTchI], Example 5.1, (v), are insulated from the indeterminacies that arise from automorphisms $\in \operatorname{Aut}_{\mathcal{F}^{\vdash \times \mu}}\left(\log \left({ }^{\alpha} \mathfrak{F}\right)^{\vdash \times \boldsymbol{\mu}}\right)$.

That is to say, it follows immediately from (i) that

the identity automorphism of $\left({ }^{\dagger} \mathbb{M}_{\mathrm{ORB}}^{\odot}\right)_{\alpha}$ is compatible with the collection of automorphisms of $\left(\Psi_{\mathfrak{l o g}\left(\alpha \mathcal{F}_{\underline{v}}\right)}^{\times \boldsymbol{\mu}}\right)_{\text {orb }}$ induced by arbitrary automorphisms $\in \operatorname{Aut}_{\mathcal{F} \vdash \times \boldsymbol{\mu}}\left(\log \left({ }^{\alpha} \mathfrak{F}\right)^{\vdash \times \mu}\right)$, relative to the diagram

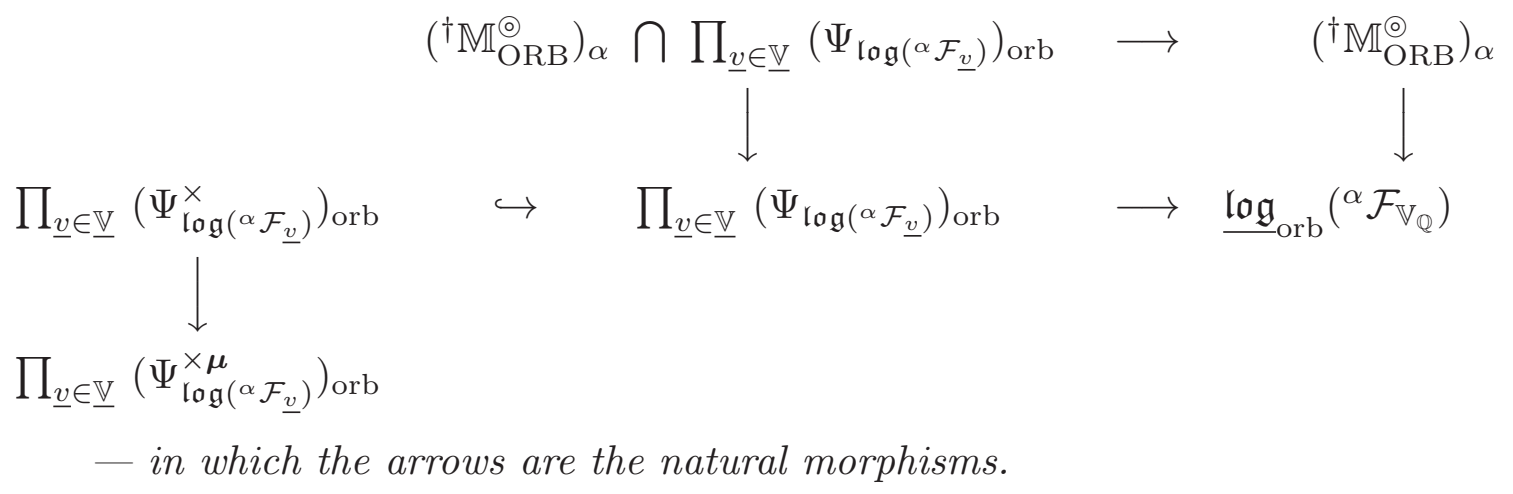

Here, we note that the significance of considering various versions of "orb" lies in the fact that collections of Galois-orbits arise naturally when one restricts the Kummer theory - i.e., the theory of Galois-stable collections of $N$-th roots of elements [for $N \geq 1$ an integer] — of copies of the number field $F_{\bmod }\left[\right.$ i.e., such as $\left({ }^{\dagger} \mathbb{M}_{\text {MOD }}^{\odot}\right)_{\alpha}$ ] to the Kummer theory of the various localizations of the number field at $\underline{v} \in \underline{\mathbb{V}}$.

\section{Remark 3.10.2.}

(i) Note that the log-Kummer correspondence of Proposition 3.10, (ii), induces isomorphisms of Frobenioids as in the first display of Proposition 3.10, (iii), precisely because the construction of " $\left({ }^{\dagger} \mathcal{F}_{\text {MOD }}^{\odot}\right)_{\alpha}$ " only involves the group " $\left({ }^{\dagger} \mathbb{M}_{\text {MOD }}^{\odot}\right) \alpha$ ", together with the collection of subquotients of its perfection indexed by $\mathbb{V}[\mathrm{cf}$. Proposition 3.7, (i); Remarks 3.6.1, 3.9.2]. By contrast, the construction of "( $\left.{ }^{\dagger} \mathcal{F}_{\mathfrak{m o d}}^{\odot}\right)_{\alpha}$ " also involves the local monoids " $\Psi_{\mathfrak{l o g}\left({ }^{A, \alpha} \mathcal{F}_{\underline{v}}\right)} \subseteq \log \left({ }^{A, \alpha} \mathcal{F}_{\underline{v}}\right)$ " in an essential way [cf. Proposition 3.7, (ii)]. These local monoids are subject to a somewhat more complicated "log-Kummer correspondence" [cf. Proposition 3.5, (ii)] that revolves around "upper semi-compatibility", i.e., in a word, one-sided inclusions, as opposed to precise equalities. The imprecise nature of such one-sided inclusions is incompatible with the construction of " $\left({ }^{\dagger} \mathcal{F}_{\mathfrak{m o d}}^{\odot}\right)_{\alpha}$ ". In particular, one cannot construct log-linkcompatible isomorphisms of Frobenioids for " $\left({ }^{\dagger} \mathcal{F}_{\mathfrak{m o d}}^{\odot}\right)_{\alpha}$ " as in the first display of Proposition 3.10, (iii). 
(ii) The precise compatibility of " $\mathcal{F}_{\text {MOD }}^{\odot}$ " with the log-links of the LGPGaussian log-theta-lattice [cf. the discussion of (i); the first "mutual compatibility" of Proposition 3.10, (iii)] makes it more suited [i.e., by comparison to " $\mathcal{F}_{\mathfrak{m o d}}^{\odot}$ "] to the task of computing the Kummer-detachment indetermacies [cf. Remark 1.5.4, (i), (iii)] that arise when one attempts to pass from the Frobenius-like structures constituted by the global portion of the domain of the $\Theta_{\mathrm{LGP}}^{\times \mu}$-links of the LGPGaussian log-theta-lattice to corresponding étale-like structures. That is to say, the mutual compatibility of the isomorphisms

$$
n, m \mathfrak{F}_{\mathrm{LGP}}^{\Vdash \perp} \stackrel{\sim}{\rightarrow} \mathfrak{F}^{\Vdash \perp}\left({ }^{n, \circ} \mathcal{H} \mathcal{T}^{\mathcal{D}-\Theta^{ \pm \mathrm{ell}} \mathrm{NF}}\right)_{\mathrm{LGP}}
$$

of the second display of Proposition 3.10, (iii), asserts, in effect, that such Kummerdetachment indeterminacies do not arise. This is precisely the reason why we wish to work with the LGP-, as opposed to the $\mathfrak{l g p}$-, Gaussian log-theta lattice [cf. Remark 3.8.1]. On the other hand, the essentially multiplicative nature of " $\mathcal{F}_{\text {MOD }}^{\odot}$ " [cf. Remark 3.6.2, (ii)] makes it ill-suited to the task of computing the étale-transport indeterminacies [cf. Remark 1.5.4, (i), (ii)] that occur as one passes between distinct arithmetic holomorphic structures on opposite sides of a $\Theta_{\mathrm{LGP}}^{\times \boldsymbol{\mu}}-$ link.

(iii) By contrast, whereas the additive nature of the local modules [i.e., local fractional ideals] that occur in the construction of " $\mathcal{F}_{\mathfrak{m o d}}^{\odot}$ " renders " $\mathcal{F}_{\mathfrak{m o d}}^{\odot}$ " ill-suited to the computation of Kummer-detachment indeterminacies [cf. the discussion of (i), (ii)], the close relationship [cf. Proposition 3.9, (i), (ii), (iii)] of these local modules to the mono-analytic log-shells that are coric with respect to the $\Theta_{\mathrm{LGP}}^{\times \boldsymbol{\mu}}$-link [cf. Theorem 1.5, (iv); Remark 3.8.2] renders " $\mathcal{F}_{\mathfrak{m o d}}^{\odot}$ " well-suited to the computation of the étale-transport indeterminacies that occur as one passes between distinct arithmetic holomorphic structures on opposite sides of a $\Theta_{\mathrm{LGP}}^{\times \boldsymbol{\mu}}$-link. That is to say, although various distortions of these local modules arise as a result of both [the Kummer-detachment indeterminacies constituted by] the local "upper semicompatibility" of Proposition 3.5, (ii), and [the étale-transport indeterminacies constituted by] the discrepancy between local holomorphic and mono-analytic integral structures [cf. Remark 3.9.1, (i), (ii)], one may nevertheless compute i.e., if one takes into account the various distortions that occur, "estimate" the global arithmetic degrees of objects of " $\mathcal{F}_{\mathfrak{m o d}}^{\odot}$ " by computing log-volumes [cf. Proposition 3.9, (iii)], which are bi-coric, i.e., coric with respect to both the

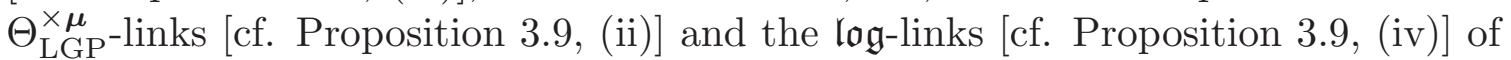
the LGP-Gaussian log-theta-lattice. This computability is precisely the topic of Corollary 3.12 below. On the other hand, the issue of obtaining concrete estimates will be treated in [IUTchIV].

(iv) The various properties of " $\mathcal{F}_{\text {MOD }}^{\odot}$ " and " $\mathcal{F}_{\mathfrak{m o d}}^{\odot}$ " discussed in (i), (ii), (iii) above are summarized in Fig. 3.2 below. In this context, it is of interest to observe that the natural isomorphisms of Frobenioids

$$
\mathcal{F}_{\mathfrak{m} \mathfrak{o d}}^{\odot}\left({ }^{n, \circ} \mathcal{H} \mathcal{T}^{\mathcal{D}-\Theta^{ \pm \mathrm{ell}} \mathrm{NF}}\right)_{\alpha} \stackrel{\sim}{\rightarrow} \mathcal{F}_{\mathrm{MOD}}^{\odot}\left({ }^{n, \circ} \mathcal{H} \mathcal{T}^{\mathcal{D}-\Theta^{ \pm \mathrm{ell}} \mathrm{NF}}\right)_{\alpha}
$$

as well as the resulting isomorphisms of $\mathcal{F}^{\Vdash}$-prime-strips

$$
\mathfrak{F}^{\Vdash}\left({ }^{n, o} \mathcal{H} \mathcal{T}^{\mathcal{D}-\Theta^{ \pm e l l} \mathrm{NF}}\right)_{\mathrm{LGP}} \stackrel{\sim}{\rightarrow} \mathfrak{F}^{\Vdash}\left({ }^{n, o} \mathcal{H} \mathcal{T}^{\mathcal{D}-\Theta^{ \pm \mathrm{ell}} \mathrm{NF}}\right)_{\mathfrak{l g p}}
$$


of Proposition 3.10, (i), play the highly nontrivial role of relating [cf. the discussion

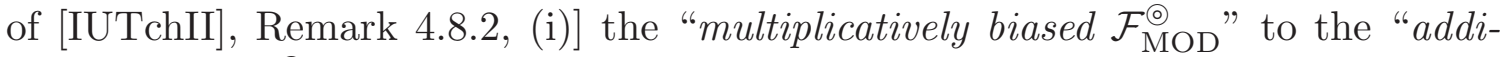
tively biased $\mathcal{F}_{\mathfrak{m o d}}^{\odot}$ " by means of the global ring structure of the number field $\overline{\mathbb{M}}_{\text {mod }}^{\odot}\left({ }^{n, o} \mathcal{H} \mathcal{T}^{\mathcal{D}-\Theta^{ \pm e l l} \mathrm{NF}}\right)_{\alpha}=\overline{\mathbb{M}}_{\mathrm{MOD}}^{\odot}\left({ }^{n, \circ} \mathcal{H} \mathcal{T}^{\mathcal{D}-\Theta^{ \pm e l l} \mathrm{NF}}\right)_{\alpha}$. A similar statement holds concerning the tautological isomorphism of $\mathcal{F}^{\Vdash}$-prime-strips ${ }^{\dagger} \mathfrak{F}_{\text {LGP }}^{\Vdash} \stackrel{\sim}{\rightarrow}{ }^{\dagger} \mathfrak{F}_{\mathfrak{l g p}}^{\|}$of Proposition 3.7, (iv).

\begin{tabular}{|c|c|}
\hline $\mathcal{F}_{\mathrm{MOD}}^{\odot} / \underline{\text { LGP-structures }}$ & $\mathcal{F}_{\mathfrak{m o d}}^{\odot} /$ lghp-structures \\
\hline $\begin{array}{c}\text { biased toward } \\
\text { multiplicative structures }\end{array}$ & $\begin{array}{l}\text { biased toward } \\
\text { additive structures }\end{array}$ \\
\hline 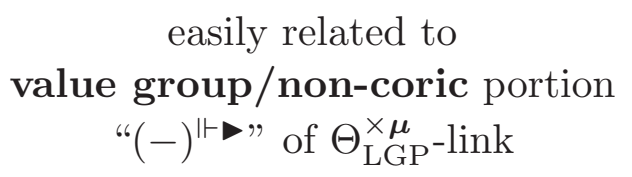 & $\begin{array}{l}\text { easily related to unit group/coric } \\
\text { portion "(- })^{\vdash \times \mu " ~ o f ~} \Theta_{\mathrm{LGP}}^{\times \boldsymbol{\mu}} / \Theta_{\mathfrak{l g p}}^{\times \mu}-l i n k \text {, } \\
\text { i.e., mono-analytic log-shells }\end{array}$ \\
\hline $\begin{array}{l}\text { admits } \\
\text { precise log-Kummer } \\
\text { correspondence }\end{array}$ & $\begin{array}{c}\text { only admits } \\
\text { "upper semi-compatible" } \\
\text { log-Kummer correspondence }\end{array}$ \\
\hline $\begin{array}{l}\text { rigid, but not suited } \\
\text { to explicit computation }\end{array}$ & $\begin{array}{l}\text { subject to substantial distortion, } \\
\text { but suited to explicit estimates }\end{array}$ \\
\hline
\end{tabular}

Fig. 3.2: $\mathcal{F}_{\mathrm{MOD}}^{\odot} /$ LGP-structures versus $\mathcal{F}_{\mathfrak{m o d}}^{\odot} /$ lgp-structures

We are now ready to discuss the main theorem of the present series of papers.

Theorem 3.11. (Multiradial Algorithms via LGP-Monoids/Frobenioids) Fix a collection of initial $\Theta$-data

$$
\left(\bar{F} / F, X_{F}, l, \underline{C}_{K}, \underline{\mathbb{V}}, \underline{\epsilon}\right)
$$

as in [IUTchI], Definition 3.1. Let

$$
\left\{{ }^{n, m} \mathcal{H} \mathcal{T}^{\Theta^{ \pm e l l} \mathrm{NF}}\right\}_{n, m \in \mathbb{Z}}
$$

be a collection of distinct $\Theta^{ \pm \text {ell }} \mathbf{N F - H o d g e ~ t h e a t e r s ~ [ r e l a t i v e ~ t o ~ t h e ~ g i v e n ~ i n i t i a l ~}$ $\Theta$-data] - which we think of as arising from an LGP-Gaussian log-theta-lattice [cf. Definition 3.8, (iii)]. For each $n \in \mathbb{Z}$, write

$$
{ }^{n, \circ} \mathcal{H} \mathcal{T}^{\mathcal{D}-\Theta^{ \pm \mathrm{ell}} \mathrm{NF}}
$$


for the $\mathcal{D}-\Theta^{ \pm \text {ell }} N F$-Hodge theater determined, up to isomorphism, by the various ${ }^{n, m} \mathcal{H} \mathcal{T}^{\Theta^{ \pm e l l} \mathrm{NF}}$, where $m \in \mathbb{Z}$, via the vertical coricity of Theorem 1.5 , (i) [cf. Remark 3.8.2].

(i) (Multiradial Representation) Consider the procession of $\mathcal{D}^{\vdash}$-primestrips $\operatorname{Prc}\left({ }^{n, \circ} \mathfrak{D}_{T}^{\vdash}\right)$

$$
\left\{{ }^{n, \circ} \mathfrak{D}_{0}^{\vdash}\right\} \hookrightarrow\left\{{ }^{n, \circ} \mathfrak{D}_{0}^{\vdash},{ }^{n, \circ} \mathfrak{D}_{1}^{\vdash}\right\} \hookrightarrow \ldots \hookrightarrow\left\{{ }^{n, \circ} \mathfrak{D}_{0}^{\vdash},{ }^{n, \circ} \mathfrak{D}_{1}^{\vdash}, \ldots,{ }^{n, \circ} \mathfrak{D}_{l^{ \pm}}^{\vdash}\right\}
$$

obtained by applying the natural functor of [IUTchI], Proposition 6.9, (ii), to [the $\mathcal{D}-\Theta^{ \pm}$-bridge associated to ${ }^{n, 0} \mathcal{H} \mathcal{T}^{\mathcal{D}-\Theta^{ \pm \mathrm{ell}} \mathrm{NF}}$. Consider also the following data:

(a) for $\underline{\mathbb{V}} \ni \underline{v}\left|v_{\mathbb{Q}}, j \in\right| \mathbb{F}_{l} \mid$, the topological modules and mono-analytic integral structures

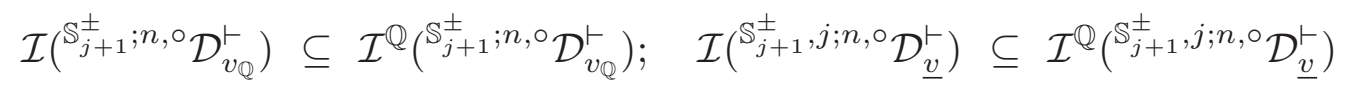

- where the notation "; $n, \circ$ " denotes the result of applying the construction in question to the case of $\mathcal{D}^{\vdash}$-prime-strips labeled " $n, \circ$ " — of Proposition 3.2, (ii) [cf. also the notational conventions of Proposition 3.4, (ii)], which we regard as equipped with the procession-normalized monoanalytic log-volumes of Proposition 3.9, (ii);

(b) for $\underline{\mathbb{V}}^{\text {bad }} \ni \underline{v}$, the splitting monoid

$$
\Psi_{\mathrm{LGP}}^{\perp}\left({ }^{n, \circ} \mathcal{H} \mathcal{T}^{\mathcal{D}-\Theta^{ \pm \mathrm{ell}} \mathrm{NF}}\right)_{\underline{v}}
$$

of Proposition 3.5, (ii), (c) [cf. also the notation of Proposition 3.5, (i)], which we regard - via the natural poly-isomorphisms

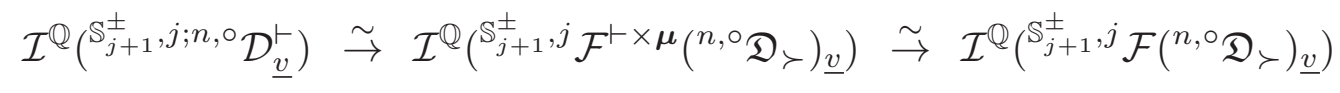

for $j \in \mathbb{F}_{l}^{*}[c f$. Proposition 3.2, (i), (ii)] - as a subset of

$$
\prod_{j \in \mathbb{F}_{l}^{*}} \mathcal{I}^{\mathbb{Q}}\left(\mathbb{S}_{j+1}^{ \pm}, j ; n, \circ \mathcal{D}_{\underline{v}}^{\vdash}\right)
$$

equipped with a(n) [multiplicative] action on $\prod_{j \in \mathbb{F}_{l}^{*}} \mathcal{I}^{\mathbb{Q}}\left(\mathbb{S}_{j+1}^{ \pm}, j ; n,{ }^{\circ} \mathcal{D}_{\underline{v}}^{+}\right)$;

(c) for $j \in \mathbb{F}_{l}^{*}$, the number field

$$
\begin{aligned}
& \overline{\mathbb{M}}_{\mathrm{MOD}}^{\odot}\left({ }^{n, o} \mathcal{H} \mathcal{T}^{\mathcal{D}-\Theta^{ \pm \mathrm{ell}} \mathrm{NF}}\right)_{j}=\overline{\mathbb{M}}_{\mathrm{mod}}^{\odot}\left({ }^{n, o} \mathcal{H} \mathcal{T}^{\mathcal{D}-\Theta^{ \pm \mathrm{ell}} \mathrm{NF}}\right)_{j} \\
& \subseteq \mathcal{I}^{\mathbb{Q}}\left(\mathbb{S}_{j+1}^{ \pm} ; n, \circ \mathcal{D}_{\mathbb{V}_{\mathbb{Q}}}^{\vdash}\right) \stackrel{\text { def }}{=} \prod_{v_{\mathbb{Q}} \in \mathbb{V}_{\mathbb{Q}}} \mathcal{I}^{\mathbb{Q}}\left({ }^{\mathbb{S}_{j+1}^{ \pm} ; n, o} \mathcal{D}_{v_{\mathbb{Q}}}^{\vdash}\right)
\end{aligned}
$$

[cf. the natural isomorphisms discussed in (b); Proposition 3.9, (iii); Proposition 3.10, (i)], together with natural isomorphisms between the associated global non-realified/ realified Frobenioids

$$
\mathcal{F}_{\mathrm{MOD}}^{\odot}\left({ }^{n, \circ} \mathcal{H} \mathcal{T}^{\mathcal{D}-\Theta^{ \pm \mathrm{ell}} \mathrm{NF}}\right)_{j} \stackrel{\sim}{\rightarrow} \mathcal{F}_{\mathfrak{m} \mathfrak{o d}}^{\odot}\left({ }^{n, \circ} \mathcal{H} \mathcal{T}^{\mathcal{D}-\Theta^{ \pm \mathrm{ell}} \mathrm{NF}}\right)_{j}
$$




$$
\mathcal{F}_{\mathrm{MOD}}^{\odot \mathbb{R}}\left({ }^{n, \circ} \mathcal{H} \mathcal{T}^{\mathcal{D}-\Theta^{ \pm \mathrm{ell}} \mathrm{NF}}\right)_{j} \stackrel{\sim}{\rightarrow} \mathcal{F}_{\mathfrak{m o d}}^{\odot \mathbb{R}}\left({ }^{n, \circ} \mathcal{H} \mathcal{T}^{\mathcal{D}-\Theta^{ \pm \mathrm{ell}} \mathrm{NF}}\right)_{j}
$$

[cf. Proposition 3.10, (i)], whose associated "global degrees" may be computed by means of the log-volumes of (a) [cf. Proposition 3.9, (iii)].

Write

$$
{ }^{n,{ }^{\circ}} \mathfrak{R}^{\mathrm{LGP}}
$$

for the collection of data (a), (b), (c) regarded up to indeterminacies of the following two types:

(Ind1) the indetermacies induced by the automorphisms of the procession of $\mathcal{D}^{\vdash}$-prime-strips $\operatorname{Prc}\left({ }^{n, \circ} \mathfrak{D}_{T}^{\vdash}\right)$;

$\left(\right.$ Ind2) for each $v_{\mathbb{Q}} \in \mathbb{V}_{\mathbb{Q}}^{\text {non }}$ (respectively, $v_{\mathbb{Q}} \in \mathbb{V}_{\mathbb{Q}}^{\text {arc }}$ ), the indeterminacies induced by the action of independent copies of Ism [cf. Proposition 1.2, (vi)] (respectively, copies of each of the automorphisms of order 2 whose orbit constitutes the poly-automorphism discussed in Proposition 1.2, (vii)) on each of the direct summands of the $j+1$ factors appearing in the tensor

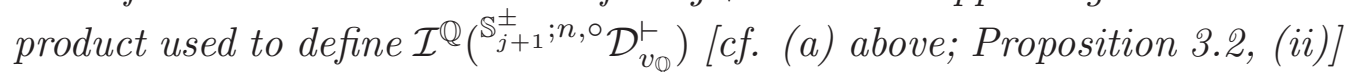
- where we recall that the cardinality of the collection of direct summands is equal to the cardinality of the set of $\underline{v} \in \underline{\mathbb{V}}$ that lie over $v_{\mathbb{Q}}$.

Then ${ }^{n, \circ} \mathfrak{R}^{\mathrm{LGP}}$ may be constructed via an algorithm in the procession of $\mathcal{D}^{\vdash}$-primestrips $\operatorname{Prc}\left({ }^{n, \circ} \mathfrak{D}_{T}^{\vdash}\right)$ that is functorial with respect to isomorphisms of processions of $\mathcal{D}^{\vdash}$-prime-strips. For $n, n^{\prime} \in \mathbb{Z}$, the permutation symmetries of the étalepicture discussed in [IUTchI], Corollary 6.10, (iii); [IUTchII], Corollary 4.11, (ii), (iii) [cf. also Corollary 2.3, (ii); Remark 3.8.2, of the present paper], induce compatible poly-isomorphisms

$$
\operatorname{Prc}\left({ }^{n, \circ} \mathfrak{D}_{T}^{\vdash}\right) \stackrel{\sim}{\rightarrow} \operatorname{Prc}\left({ }^{n^{\prime}, \circ} \mathfrak{D}_{T}^{\vdash}\right) ; \quad{ }^{n, \circ} \mathfrak{R}^{\mathrm{LGP}} \stackrel{\sim}{\rightarrow} n^{\prime},{ }^{\circ} \mathfrak{R}^{\mathrm{LGP}}
$$

which are, moreover, compatible with the poly-isomorphisms

$$
n, \circ \mathfrak{D}_{0}^{\vdash} \stackrel{\sim}{\rightarrow} n^{\prime}, \circ \mathfrak{D}_{0}^{\vdash}
$$

induced by the bi-coricity poly-isomorphisms of Theorem 1.5, (iii) [cf. also [IUTchII], Corollaries 4.10, (iv); 4.11, (i)].

(ii) (log-Kummer Correspondence) For $n, m \in \mathbb{Z}$, the Kummer isomorphisms of labeled data

$$
\begin{gathered}
\Psi_{\mathrm{cns}}\left({ }^{n, m} \mathfrak{F}_{\succ}\right)_{t} \stackrel{\sim}{\rightarrow} \Psi_{\mathrm{cns}}\left({ }^{n, \circ} \mathfrak{D}_{\succ}\right)_{t} \\
\left\{\pi_{1}\left({ }^{n,{ }^{\circ}} \mathcal{D}^{\circledast}\right) \curvearrowright{ }^{n, m} \overline{\mathbb{M}}^{\odot}\right\} \stackrel{\sim}{\rightarrow}\left\{\pi_{1}\left({ }^{n,{ }^{\circ}} \mathcal{D}^{\circledast}\right) \curvearrowright \overline{\mathbb{M}}^{\odot}\left({ }^{n, \circ} \mathcal{D}^{\odot}\right)\right\} \\
\left({ }^{n, m} \overline{\mathbb{M}}_{\text {mod }}^{\odot}\right)_{j} \stackrel{\sim}{\rightarrow} \overline{\mathbb{M}}_{\text {mod }}^{\odot}\left({ }^{n, \circ} \mathcal{D}^{\odot}\right)_{j} ; \quad\left({ }^{n, m} \overline{\mathbb{M}}_{\text {orb }}^{\odot}\right)_{j} \stackrel{\sim}{\rightarrow} \overline{\mathbb{M}}_{\text {orb }}^{\odot}\left({ }^{n, o} \mathcal{D}^{\odot}\right)_{j}
\end{gathered}
$$

- where $t \in \operatorname{LabCusp}^{ \pm}\left({ }^{n, \circ} \mathfrak{D}_{\succ}\right)-$ of [IUTchII], Corollary 4.6, (iii); [IUTchII], Corollary 4.8, (i), (ii) [cf. also Propositions 3.5, (i); 3.10, (i), of the present paper] induce isomorphisms between the vertically coric data (a), (b), (c) of (i) and 
the corresponding data arising from each $\Theta^{ \pm \mathrm{ell}} N F$-Hodge theater ${ }^{n, m} \mathcal{H} \mathcal{T}^{\Theta^{ \pm \mathrm{ell}} \mathrm{NF}}$, i.e.:

(a) for $\underline{\mathbb{V}} \ni \underline{v}\left|v_{\mathbb{Q}}, j \in\right| \mathbb{F}_{l} \mid$, isomorphisms with local mono-analytic tensor packets and their $\mathbb{Q}$-spans

$$
\begin{aligned}
& \mathcal{I}\left({ }^{\mathbb{S}_{j+1}^{ \pm} ; n, m} \mathcal{F}_{v_{\mathbb{Q}}}\right) \stackrel{\sim}{\rightarrow} \mathcal{I}\left({ }^{\mathbb{S}_{j+1}^{ \pm} ; n, m} \mathcal{F}_{v_{\mathbb{Q}}}^{+\times \mu}\right) \stackrel{\sim}{\rightarrow} \mathcal{I}\left({ }^{\mathbb{S}_{j+1}^{ \pm} ; n, \circ} \mathcal{D}_{v_{\mathbb{Q}}}^{\vdash}\right)
\end{aligned}
$$

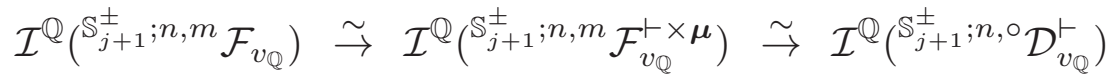

$$
\begin{aligned}
& \mathcal{I}\left({ }^{\mathbb{S}_{j+1}^{ \pm}, j ; n, m} \mathcal{F}_{\underline{v}}\right) \stackrel{\sim}{\rightarrow} \mathcal{I}\left({ }^{\mathbb{S}_{j+1}^{ \pm}, j ; n, m} \mathcal{F}_{\underline{v}}^{\vdash \times \mu}\right) \stackrel{\sim}{\rightarrow} \mathcal{I}\left({ }^{\mathbb{S}_{j+1}^{ \pm}, j ; n,{ }^{\circ}} \mathcal{D}_{\underline{v}}^{\vdash}\right)
\end{aligned}
$$

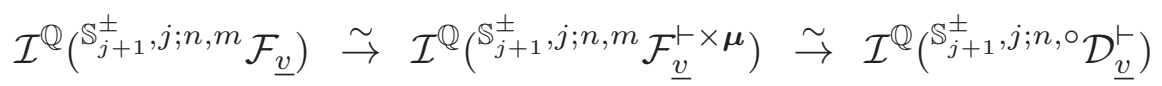

[cf. Propositions 3.2, (i), (ii); 3.4, (ii); 3.5, (i)], all of which are compatible with the respective log-volumes [cf. Proposition 3.9, (ii)];

(b) for $\underline{\mathbb{V}}^{\text {bad }} \ni \underline{v}$, isomorphisms of splitting monoids

$$
\Psi_{\mathcal{F}_{\mathrm{LGP}}}^{\perp}\left({ }^{n, m} \mathcal{H} \mathcal{T}^{\Theta^{ \pm \mathrm{ell}} \mathrm{NF}}\right)_{\underline{v}} \stackrel{\sim}{\rightarrow} \Psi_{\mathrm{LGP}}^{\perp}\left({ }^{n, o} \mathcal{H} \mathcal{T}^{\mathcal{D}-\Theta^{ \pm \mathrm{ell}} \mathrm{NF}}\right)_{\underline{v}}
$$

[cf. Proposition 3.5, (i); Proposition 3.5, (ii), (c)];

(c) for $j \in \mathbb{F}_{l}^{*}$, isomorphisms of number fields and non-realified/realified global Frobenioids

$$
\begin{aligned}
\left({ }^{n, m} \overline{\mathbb{M}}_{\mathrm{MOD}}^{\odot}\right)_{j} \stackrel{\sim}{\rightarrow} \overline{\mathbb{M}}_{\mathrm{MOD}}^{\odot}\left({ }^{n, o} \mathcal{H} \mathcal{T}^{\mathcal{D}-\Theta^{ \pm e l l} \mathrm{NF}}\right)_{j} ; \quad\left({ }^{n, m} \overline{\mathbb{M}}_{\mathfrak{m o d}}^{\odot}\right)_{j} \stackrel{\sim}{\rightarrow} \overline{\mathbb{M}}_{\mathfrak{m o d}}^{\odot}\left({ }^{n, o} \mathcal{H} \mathcal{T}^{\mathcal{D}-\Theta^{ \pm e l l} \mathrm{NF}}\right)_{j} \\
\left({ }^{n, m} \mathcal{F}_{\mathrm{MOD}}^{\odot}\right)_{j} \stackrel{\sim}{\rightarrow} \mathcal{F}_{\mathrm{MOD}}^{\odot}\left({ }^{n, o} \mathcal{H} \mathcal{T}^{\mathcal{D}-\Theta^{ \pm e l l} \mathrm{NF}}\right)_{j} ; \quad\left({ }^{n, m} \mathcal{F}_{\mathfrak{m o d}}^{\odot}\right)_{j} \stackrel{\sim}{\rightarrow} \mathcal{F}_{\mathfrak{m o d}}^{\odot}\left({ }^{n, o} \mathcal{H} \mathcal{T}^{\mathcal{D}-\Theta^{ \pm e l l} \mathrm{NF}}\right)_{j} \\
\left({ }^{n, m} \mathcal{F}_{\mathrm{MOD}}^{\odot \mathbb{R}}\right)_{j} \stackrel{\sim}{\rightarrow} \mathcal{F}_{\mathrm{MOD}}^{\odot \mathbb{R}}\left({ }^{n, o} \mathcal{H} \mathcal{T}^{\mathcal{D}-\Theta^{ \pm e l l} \mathrm{NF}}\right)_{j} ; \quad\left({ }^{n, m} \mathcal{F}_{\mathfrak{m o d}}^{\odot \mathbb{R}}\right)_{j} \stackrel{\sim}{\rightarrow} \mathcal{F}_{\mathfrak{m o d}}^{\odot \mathbb{R}}\left({ }^{n, o} \mathcal{H} \mathcal{T}^{\mathcal{D}-\Theta^{ \pm e l l} \mathrm{NF}}\right)_{j}
\end{aligned}
$$

which are compatible with the respective natural isomorphisms between "MOD"- and "mod"-subscripted versions [cf. Proposition 3.10, (i)]; here, the isomorphisms of the third line of the display induce isomorphisms of the global realified Frobenioid portions

$$
\begin{aligned}
& { }^{n, m} \mathcal{C}_{\mathrm{LGP}}^{\Vdash} \stackrel{\sim}{\rightarrow} \mathcal{C}_{\mathrm{LGP}}^{\Vdash}\left({ }^{n, \circ} \mathcal{H} \mathcal{T}^{\mathcal{D}-\Theta^{ \pm e l l} \mathrm{NF}}\right) ; \quad{ }^{n, m} \mathcal{C}_{\mathfrak{l g p}}^{\Vdash} \stackrel{\sim}{\rightarrow} \mathcal{C}_{\mathfrak{l g p}}^{\Vdash}\left({ }^{n, o} \mathcal{H} \mathcal{T}^{\mathcal{D}-\Theta^{ \pm e l l} \mathrm{NF}}\right) \\
& \text { of the } \mathcal{F}^{\Vdash} \text {-prime-strips }{ }^{n, m} \mathfrak{F}_{\mathrm{LGP}}^{\Vdash}, \mathfrak{F}^{\Vdash}\left({ }^{n, o} \mathcal{H} \mathcal{T}^{\mathcal{D} \text { - } \Theta^{ \pm e l l} \mathrm{NF}}\right)_{\mathrm{LGP}},{ }^{n, m} \mathfrak{F}_{\mathfrak{l g p}}^{\Vdash} \text {, and } \\
& \mathfrak{F}^{\Vdash}\left({ }^{n, o} \mathcal{H} \mathcal{T}^{\mathcal{D}-\Theta^{ \pm \mathrm{ell}} \mathrm{NF}}\right)_{\mathfrak{l g} \mathfrak{p}} \text { [cf. Propositions 3.7, (iii), (iv), (v); 3.10, (i)]. }
\end{aligned}
$$

Moreover, as one varies $m \in \mathbb{Z}$, the isomorphisms of (b), as well as the isomorphisms of the first line in the first display of (c), are mutually compatible, relative to the log-links of the n-th column of the LGP-Gaussian log-theta-lattice under consideration, in the sense that the only portions of the domains of these isomorphisms that are related to one another via the log-links consist of roots of unity [multiplication by which corresponds, via the log-link, to an "addition by zero" indeterminacy, i.e., to no indeterminacy!] - cf. Proposition 3.5, (ii), (c); Proposition 3.10, (ii). This mutual compatibility of the isomorphisms of the 
first line in the first display of (c) implies a corresponding mutual compatibility between the isomorphisms of the second and third lines in the first display of (c) that involve the subscript "MOD" [but not between the isomorphisms that involve the subscript "mod"! - cf. Proposition 3.10, (iii); Remark 3.10.2]. On the other hand, the isomorphisms of (a) are subject to a certain "indeterminacy" as follows:

(Ind3) as one varies $m \in \mathbb{Z}$, the isomorphisms of (a) are "upper semicompatible", relative to the log-links of the $n$-th column of the LGPGaussian log-theta-lattice under consideration, in a sense that involves certain natural inclusions " $\subseteq$ " at $v_{\mathbb{Q}} \in \mathbb{V}_{\mathbb{Q}}^{\text {non }}$ and certain natural surjections " $\rightarrow$ " at $v_{\mathbb{Q}} \in \mathbb{V}_{\mathbb{Q}}^{\text {arc }}-c f$. Proposition 3.5, (ii), (a), (b), for more details.

Finally, as one varies $m \in \mathbb{Z}$, the isomorphisms of (a) are [precisely!] compatible, relative to the log-links of the $n$-th column of the LGP-Gaussian log-theta-lattice under consideration, with the respective log-volumes [cf. Proposition 3.9, (iv)].

(iii) $\left(\Theta_{\mathrm{LGP}}^{\times \boldsymbol{\mu}}\right.$-Link Compatibility) The various Kummer isomorphisms of (ii) satisfy compatibility properties with the various horizontal arrows - i.e., $\Theta_{\mathrm{LGP}}^{\times \mu}$ links - of the LGP-Gaussian log-theta-lattice under consideration as follows:

(a) The first Kummer isomorphism of the first display of (ii) induces - by applying the $\mathbb{F}_{l}^{\rtimes \pm}$-symmetry of the $\Theta^{ \pm \text {ell }} N F$-Hodge theater ${ }^{n, m} \mathcal{H} \mathcal{T}^{\Theta^{ \pm e l l}} \mathrm{NF}$ - $a$ Kummer isomorphism ${ }^{n, m} \mathfrak{F}_{\triangle}^{\vdash \times \mu} \stackrel{\sim}{\rightarrow} \mathfrak{F}_{\triangle}^{\vdash \times \mu}\left({ }^{n, \circ} \mathfrak{D}_{\triangle}^{\vdash}\right)$ [cf. Theorem 1.5, (iii)]. Relative to this Kummer isomorphism, the full polyisomorphism of $\mathcal{F}^{\vdash \times \boldsymbol{\mu}}$-prime-strips

$$
\mathfrak{F}_{\triangle}^{\vdash} \times \boldsymbol{\mu}\left({ }^{n, \circ} \mathfrak{D}_{\triangle}^{\vdash}\right) \stackrel{\sim}{\rightarrow} \mathfrak{F}_{\triangle}^{\vdash} \times \boldsymbol{\mu}\left({ }^{n+1, \circ} \mathfrak{D}_{\triangle}^{\vdash}\right)
$$

is compatible with the full poly-isomorphism of $\mathcal{F}^{\vdash \times \boldsymbol{\mu}}$-prime-strips

$$
n, m \mathfrak{F}_{\triangle}^{\vdash} \times \boldsymbol{\mu} \stackrel{\sim}{\rightarrow} n+1, m \mathfrak{F}_{\triangle}^{\vdash} \times \boldsymbol{\mu}
$$

induced [cf. Theorem 1.5, (ii)] by the horizontal arrows of the LGPGaussian log-theta-lattice under consideration [cf. Theorem 1.5, (iii)].

(b) The $\mathcal{F}^{\Vdash}$-prime-strips ${ }^{n, m} \mathfrak{F}_{\text {env }}^{\Vdash}, \mathfrak{F}_{\text {env }}^{\Vdash}\left({ }^{n, \circ} \mathfrak{D}_{>}\right)$[cf. Proposition 2.1, (ii)] that appear implicitly in the construction of the local portion of the $\mathcal{F}^{\Vdash}$-primestrips $^{n, m} \mathfrak{F}_{\mathrm{LGP}}^{\Vdash}, \mathfrak{F}^{\Vdash}\left({ }^{n, o} \mathcal{H} \mathcal{T}^{\mathcal{D}-\Theta^{ \pm \text {ell }} \mathrm{NF}}\right)_{\mathrm{LGP}},{ }^{n, m} \mathfrak{F}_{\mathfrak{l g p}}^{\vdash}, \mathfrak{F}^{\Vdash}\left({ }^{n, o} \mathcal{H} \mathcal{T}^{\mathcal{D}-\Theta^{ \pm e l l} \mathrm{NF}}\right)_{\mathfrak{l g p}}$ [cf. (ii), (b), above; Proposition 3.4, (ii); Proposition 3.7, (iii), (iv); [IUTchII], Corollary 4.6, (iv)] admit natural isomorphisms of associated $\mathcal{F}^{\vdash \times \boldsymbol{\mu}}$-prime-strips ${ }^{n, m} \mathfrak{F}_{\triangle}^{\vdash \times \boldsymbol{\mu}} \stackrel{\sim}{\rightarrow} n, m \mathfrak{F}_{\text {env }}^{\vdash \times \boldsymbol{\mu}}, \quad \mathfrak{F}_{\triangle}^{\vdash \times \boldsymbol{\mu}}\left(n, \circ \mathfrak{D}_{\triangle}^{\vdash}\right) \stackrel{\sim}{\rightarrow}$ $\mathfrak{F}_{\text {env }}^{\vdash \times \boldsymbol{\mu}}\left({ }^{n, \circ} \mathfrak{D}_{>}\right)$[cf. Proposition 2.1, (vi)]. Relative to these natural isomorphisms and to the Kummer isomorphism discussed in (a) above, the full poly-isomorphism of $\mathcal{F}^{\vdash \times \mu}$-prime-strips

$$
\mathfrak{F}_{\text {env }}^{\vdash \times \mu}\left({ }^{n, \circ} \mathfrak{D}_{\triangle}^{\vdash}\right) \stackrel{\sim}{\rightarrow} \mathfrak{F}_{\text {env }}^{\vdash \times \mu}\left({ }^{n+1, \circ} \mathfrak{D}_{\triangle}^{\vdash}\right)
$$


is compatible with the full poly-isomorphism of $\mathcal{F}^{\vdash \times \boldsymbol{\mu}}$-prime-strips

$$
n, m \underset{\mathfrak{F}_{\triangle} \times \boldsymbol{\mu}}{\stackrel{\sim}{\rightarrow}}{ }^{n+1, m} \mathfrak{F}_{\triangle}^{\vdash \times \mu}
$$

induced [cf. Theorem 1.5, (ii)] by the horizontal arrows of the LGPGaussian log-theta-lattice under consideration [cf. Corollary 2.3, (iii)].

(c) Recall the data "n,o $\mathfrak{R}^{\text {bad" }}$ [cf. Corollary 2.3, ( $\left.c_{\mathfrak{R}}\right)$ ] associated to the $\mathcal{D}-\Theta^{ \pm \text {ell }} N F$-Hodge theater ${ }^{n, \circ} \mathcal{H} \mathcal{T}^{\mathcal{D}-\Theta^{ \pm \text {ell }} \mathrm{NF}}$ at $\underline{v} \in \underline{\mathbb{V}}^{\text {bad }}$ - data which appears implicitly in the construction of the local portion of the $\mathcal{F}^{\Vdash}$-primestrips $^{n, m} \mathfrak{F}_{\mathrm{LGP}}^{\Vdash}, \mathfrak{F}^{\Vdash}\left({ }^{n, o} \mathcal{H} \mathcal{T}^{\mathcal{D}-\Theta^{ \pm e l l} \mathrm{NF}}\right)_{\mathrm{LGP}},{ }^{n, m} \mathfrak{F}_{\mathfrak{l g p}}^{\Vdash}, \mathfrak{F}^{\Vdash}\left({ }^{n, o} \mathcal{H} \mathcal{T}^{\mathcal{D}-\Theta^{ \pm \text {ell }} \mathrm{NF}}\right)_{\mathfrak{l g p}}$ [cf. (ii), (b), above; Proposition 3.4, (ii); Proposition 3.7, (iii), (iv); [IUTchII], Corollary 4.6, (iv)]. This data that arises from ${ }^{n, \circ} \mathcal{H T}^{\mathcal{D}-\Theta^{ \pm \mathrm{ell}} \mathrm{NF}}$ is related to corresponding data that arises from the projective system of mono-theta environments associated to the tempered Frobenioids of the $\Theta^{ \pm \text {ell }} N F$-Hodge theater ${ }^{n, m} \mathcal{H} \mathcal{T}^{\Theta^{ \pm \text {ell }} \mathrm{NF}}$ at $\underline{v} \in \underline{\mathbb{V}}^{\text {bad }}$ via the Kummer isomorphisms and poly-isomorphisms of projective systems of mono-theta environments discussed in Proposition 2.1, (ii), (iii) [cf. also the second display of Theorem 2.2, (ii)] and Theorem 1.5, (iii) [cf. also (a), (b) above]. Relative to these Kummer isomorphisms and polyisomorphisms of projective systems of mono-theta environments, the polyisomorphism

$$
{ }^{n, \circ} \mathfrak{R}^{\mathrm{bad}} \stackrel{\sim}{\rightarrow} n+1, \circ \mathfrak{R}^{\mathrm{bad}}
$$

induced by any permutation symmetry of the étale-picture [cf. the final portion of (i) above; Corollary 2.3, (ii); Remark 3.8.2 $]^{n, o} \mathcal{H T}^{\mathcal{D}-\Theta^{ \pm \mathrm{ell}} \mathrm{NF}}$ $\stackrel{\sim}{\rightarrow} n+1,{ }^{\circ} \mathcal{H} \mathcal{T}^{\mathcal{D}-\Theta^{ \pm e l l} \mathrm{NF}}$ is compatible with the full poly-isomorphism of $\mathcal{F}^{\vdash \times \boldsymbol{\mu}}$-prime-strips

$$
n, m \mathfrak{F}_{\triangle}^{\vdash \times \mu} \stackrel{\sim}{\rightarrow} n+1, m \mathfrak{F}_{\triangle}^{\vdash \times \mu}
$$

induced [cf. Theorem 1.5, (ii)] by the horizontal arrows of the LGPGaussian log-theta-lattice under consideration [cf. Corollary 2.3, (iv)].

(d) Relative to the Kummer isomorphisms of the first display of (ii) [cf. also (a), (b) above], the poly-isomorphisms

$$
\begin{aligned}
\left\{\pi_{1}\left({ }^{n, \circ} \mathcal{D}^{\circledast}\right) \curvearrowright\right. & \left.\overline{\mathbb{M}}^{\odot}\left({ }^{n, o} \mathcal{D}^{\odot}\right)\right\} \stackrel{\sim}{\rightarrow}\left\{\pi_{1}\left({ }^{n+1, \circ} \mathcal{D}^{\circledast}\right) \curvearrowright \overline{\mathbb{M}}^{\odot}\left({ }^{n+1, \circ} \mathcal{D}^{\odot}\right)\right\} \\
& \overline{\mathbb{M}}_{\text {orb }}^{\odot}\left({ }^{n, \circ} \mathcal{D}^{\odot}\right)_{j} \stackrel{\sim}{\rightarrow} \overline{\mathbb{M}}_{\text {orb }}^{\odot}\left({ }^{n+1,{ }^{\circ}} \mathcal{D}^{\odot}\right)_{j}
\end{aligned}
$$

induced by any permutation symmetry of the étale-picture [cf. the final portion of (i) above; Corollary 2.3, (ii); Remark 3.8.2] ${ }^{n, \circ} \mathcal{H T}^{\mathcal{D}-\Theta^{ \pm e l l} \mathrm{NF}}$ $\stackrel{\sim}{\rightarrow} n+1,{ }^{\circ} \mathcal{H} \mathcal{T}^{\mathcal{D}-\Theta^{ \pm \mathrm{ell}} \mathrm{NF}}$ are compatible [cf. the discussion of Remark 3.10.1, (ii), for more details] with the full poly-isomorphism of $\mathcal{F}^{\vdash \times \boldsymbol{\mu}_{-}}$ prime-strips

$$
n, m \mathfrak{F}_{\triangle}^{\vdash \times \mu} \stackrel{\sim}{\rightarrow} n+1, m \mathfrak{F}_{\triangle}^{\vdash \times \mu}
$$

induced [cf. Theorem 1.5, (ii)] by the horizontal arrows of the LGPGaussian log-theta-lattice under consideration. 
Proof. The various assertions of Theorem 3.11 follow immediately from the definitions and the references quoted in the statements of these assertions - cf. also the various related observations of Remarks 3.11.1, 3.11.2 below.

Remark 3.11.1. One way to summarize the content of Theorem 3.11 is as follows:

Theorem 3.11 gives an algorithm for describing, up to certain relatively mild indeterminacies, the LGP-monoids [cf. Fig. 3.1] — i.e., in essence, the theta values

$$
\left\{\underline{\underline{q}}^{j^{2}}\right\}_{j=1, \ldots, l *}
$$

- which are constructed relative to the scheme/ring structure, i.e., "arithmetic holomorphic structure", associated to one vertical line [i.e., " $(n, \circ)$ " for fixed some $n \in \mathbb{Z}$ ] in the LGP-Gaussian log-theta-lattice under consideration in terms of the a priori alien arithmetic holomorphic structure of another vertical line [i.e., " $(n+1,0)$ "] in the LGP-Gaussian log-theta-lattice under consideration - cf., especially, the final portion of Theorem 3.11, (i), concerning functoriality and compatibility with the permutation symmetries of the étale-picture.

This point of view is consistent with the point of view of the discussion of Remark 1.5.4; [IUTchII], Remark 3.8.3, (iii).

\section{Remark 3.11.2.}

(i) In Theorem 3.11, (i), we do not apply the formalism or language developed in [IUTchII], §1, for discussing multiradiality. Nevertheless, the approach taken in Theorem 3.11, (i) - i.e., by regarding the collection of data (a), (b), (c) up to the indeterminacies given by (Ind1), (Ind2) - to constructing "multiradial representations" amounts, in essence, to a special case of the tautological approach to constructing multiradial environments discussed in [IUTchII], Example 1.9, (ii). That is to say, this tautological approach is applied to the vertically coric constructions of Proposition 3.5, (i); 3.10, (i), which, a priori, are uniradial in the sense that they depend, in an essential way, on the arithmetic holomorphic structure constituted by a particular vertical line - i.e., " $(n, \circ)$ " for fixed some $n \in \mathbb{Z}$ - in the LGP-Gaussian log-theta-lattice under consideration.

(ii) One important underlying aspect of the tautological approach to multiradiality discussed in (i) is the treatment of the various labels that occur in the multiplicative and additive combinatorial Teichmüller theory associated to the $\mathcal{D}-\Theta^{ \pm \text {ell }} \mathrm{NF}$-Hodge theater ${ }^{n, o} \mathcal{H} \mathcal{T}^{\mathcal{D}-\Theta^{ \pm \text {ell }} \mathrm{NF}}$ under consideration [cf. the theory of [IUTchI], §4, 66 . The various transitions between types of labels is illustrated in Fig. 3.3 below. Here, we recall that:

(a) the passage from the $\mathbb{F}_{l}^{\rtimes \pm}$-symmetry to labels $\in \mathbb{F}_{l}$ forms the content of the associated $\mathcal{D}-\Theta^{ \pm \text {ell }}$-Hodge theater [cf. [IUTchI], Remark 6.6.1]; 
(b) the passage from labels $\in \mathbb{F}_{l}$ to labels $\in\left|\mathbb{F}_{l}\right|$ forms the content of the functorial algorithm of [IUTchI], Proposition 6.7;

(c) the passage from labels $\in\left|\mathbb{F}_{l}\right|$ to \pm -processions forms the content of [IUTchI], Proposition 6.9, (ii);

(d) the passage from the $\mathbb{F}_{l}^{*}$-symmetry to labels $\in \mathbb{F}_{i}^{*}$ forms the content of the associated $\mathcal{D}-\Theta N F$-Hodge theater [cf. [IUTchI], Remark 4.7.2, (i)];

(e) the passage from labels $\in \mathbb{F}_{l}^{*}$ to $*$-processions forms the content of [IUTchI], Proposition 4.11, (ii);

(f) the compatibility between *-processions and \pm -processions, relative to the natural inclusion of labels $\mathbb{F}_{l}^{*} \hookrightarrow\left|\mathbb{F}_{l}\right|$, forms the content of [IUTchI], Proposition 6.9, (iii).

Here, we observe in passing that, in order to perform these various transitions, it is absolutely necessary to work with all of the labels in $\mathbb{F}_{l}$ or $\left|\mathbb{F}_{l}\right|$, i.e., one does not have the option of "arbitrarily omitting certain of the labels" [cf. the discussion of [IUTchII], Remark 2.6.3; [IUTchII], Remark 3.5.2]. Also, in this context, it is important to note that there is a fundamental difference between the labels $\in$ $\mathbb{F}_{l},\left|\mathbb{F}_{l}\right|, \mathbb{F}_{l}^{*}$ - which are essentially arithmetic holomorphic in the sense that they depend, in an essential way, on the various local and global arithmetic fundamental groups involved - and the index sets of the mono-analytic \pm -processions that appear in the multiradial representation of Theorem 3.11, (i). Indeed, these index sets are just "naked sets" which are determined, up to isomorphism, by their cardinality. In particular,

the construction of these index sets is independent of the various arithmetic holomorphic structures involved.

Indeed, it is precisely this property of these index sets that renders them suitable for use in the construction of the multiradial representations of Theorem 3.11, (i). As discussed in [IUTchI], Proposition 6.9, (i), for $j \in\{0, \ldots, l *\}$, there are precisely $j+1$ possibilities for the "element labeled $j$ " in the index set of cardinality $j+1$; this leads to a total of $\left(l^{*}+1\right) !=l^{ \pm}$! possibilities for the "label indentification" of elements of index sets of capsules appearing in the mono-analytic \pm -processions of Theorem 3.11, (i). Finally, in this context, it is of interest to recall that the "rougher approach to symmetrization" that arises when one works with mono-anlytic processions is ["downward"] compatible with the finer arithmetically holomorphic approach to symmetrization that arises from the $\mathbb{F}_{l}^{\rtimes \pm}$-symmetry [cf. [IUTchII], Remark 3.5.3; [IUTchII], Remark 4.5.2, (ii); [IUTchII], Remark 4.5.3, (ii)].

(iii) Observe that the "Kummer isomorphism of global realified Frobenioids" that appears in the theory of [IUTchII], $\S 4$ - i.e., more precisely, the various versions of the isomorphism of Frobenioids " $\ddagger \mathcal{C}^{\Vdash} \stackrel{\sim}{\rightarrow} \mathcal{D}^{\Vdash}\left({ }^{\ddagger} \mathfrak{D}^{\vdash}\right)$ " discussed in [IUTchII], Corollary 4.6, (ii), (v) — is constructed by considering isomorphisms between local value groups obtained by forming the quotient of the multiplicative groups associated to the various local fields that appear by the subgroups of 
local units [cf. [IUTchII], Propositions 4.2, (ii); 4.4, (ii)]. In particular, such "Kummer isomorphisms" fail to give rise to a "log-Kummer correspondence", i.e., they fail to satisfy mutual compatibility properties of the sort discussed in the final portion of Theorem 3.11, (ii). Indeed, as discussed in Remark 1.2.3, (i) [cf. also [IUTchII], Remark 1.12.2, (iv)], at $\underline{v} \in \underline{\mathbb{V}}^{\text {non }}$, the operation of forming a multiplicative quotient by local units corresponds, on the opposite side of the log-link, to forming an additive quotient by the submodule obtained as the $p_{\underline{\underline{v}}}$-adic logarithm of these local units. This is precisely why, in the context of Theorem 3.11, (ii), we wish to work with the global non-realified/realified Frobenioids " $\mathcal{F}_{\text {MOD }}^{\odot}$ ", " $\mathcal{F}_{\text {MOD }}^{\odot \mathbb{R}}$ " that arise from copies of " $F_{\text {mod" }}$ " which satisfy a "log-Kummer correspondence", as described in the final portion of Theorem 3.11, (ii) [cf. the discussion of Remark 3.10.2]. On the other hand, the pathologies/indeterminacies that arise from working with global arithmetic line bundles by means of various local data at $\underline{v} \in \underline{\mathbb{V}}$ in the context of the log-link are formalized via the theory of the global Frobenioids " $\mathcal{F}_{\mathfrak{m o d}}^{\odot}$ ", together with the "upper semi-compatibility" of local units discussed in the final portion of Theorem 3.11, (ii) [cf. also the discussion of Remark 3.10.2].

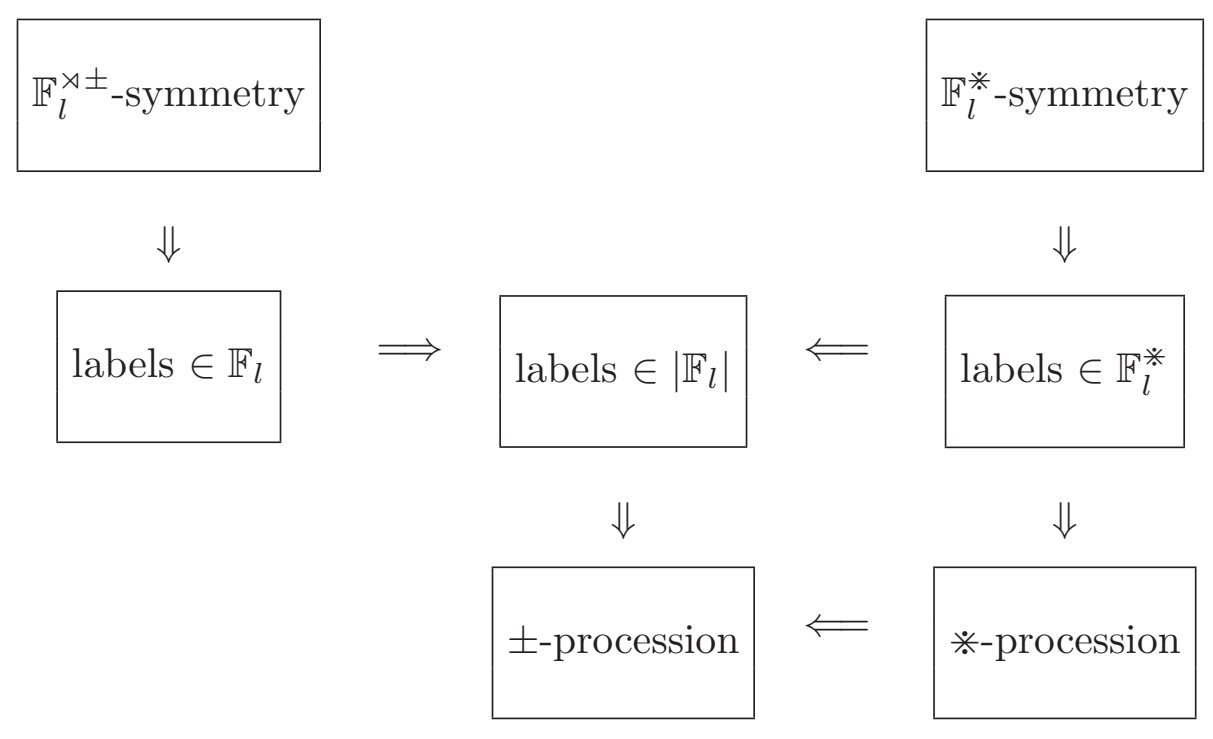

Fig. 3.3: Transitions from symmetries to labels to processions in a $\Theta^{ \pm \text {ell }} \mathrm{NF}$-Hodge theater

(iv) In the context of the discussion of global realified Frobenioids given in (iii), we observe that, in the case of the global realified Frobenioids [constructed by means of " $\mathcal{F}_{\text {MOD }}^{\odot \mathbb{R}}$ "!] that appear in the $\mathcal{F}^{\Vdash}$-prime-strips ${ }^{n, m} \mathfrak{F}_{\text {LGP }}^{\Vdash}, \mathfrak{F}^{\Vdash}\left({ }^{n, o} \mathcal{H} \mathcal{T}^{\mathcal{D}-\Theta^{ \pm \text {ell }}}{ }^{\text {NF }}\right)_{\text {LGP }}$ [cf. Theorem 3.11, (ii), (c)], the various localization functors that appear [i.e., the various " $\rho_{\underline{v}}$ " of [IUTchI], Definition 5.2 , (iv); cf. also the isomorphisms of the second display of [IUTchII], Corollary 4.6, (v)] may be reconstructed, in the spirit of the discussion of Remark 3.9.2, "by considering the effect of multiplication by elements of the [non-realified] global monoids under consideration on the logvolumes of the various local mono-analytic tensor packets that appear". [We leave the routine details to the reader.] This reconstructibility, together with the mutual incompatibilities observed in (iii) above that arise when one attempts to work simultaneously with log-shells and with the splitting monoids of the $\mathcal{F}^{\Vdash}$-prime-strip 
$n, m \mathfrak{F}_{\mathrm{LGP}}^{\vdash}$ at $\underline{v} \in \underline{\mathbb{V}}^{\text {good }}$, are the primary reasons for our omission of the the splitting monoids at $\underline{v} \in \underline{\mathbb{V}}^{\text {good }}$ from the statement of Theorem 3.11 [cf. Theorem 3.11, (i), (b); Theorem 3.11, (ii), (b); Theorem 3.11, (iii), (c), in the case of $\left.\underline{v} \in \underline{\mathbb{V}}^{\text {bad }}\right]$.

Remark 3.11.3. Before proceeding, we pause to discuss the relationship between the log-Kummer correspondence of Theorem 3.11, (ii), and the $\Theta_{\mathrm{LGP}}^{\times \boldsymbol{\mu}}$-link compatibility of Theorem 3.11, (iii).

(i) First, we recall [cf. Remarks 1.4.1, (i); 3.8.2] that the various squares that appear in the [LGP-Gaussian] log-theta-lattice are far from being [1-]commutative! On the other hand, the bi-coricity of $\mathcal{F}^{\vdash \times \mu}$-prime-strips and mono-analytic logshells discussed in Theorem 1.5, (iii), (iv), may be intepreted as the statement that

the various squares that appear in the [LGP-Gaussian] log-theta-lattice are in fact [1-] commutative with respect to [the portion of the data associated to each "•" in the log-theta-lattice that is constituted by] these bi-coric $\mathcal{F}^{\vdash \times \mu}$-prime-strips and mono-analytic log-shells.

(ii) Next, let us observe that in order to relate both the unit and value group portions of the domain and codomain of the $\Theta_{\mathrm{LGP}}^{\times \mu}$-link corresponding to adjacent vertical lines - i.e., $(n-1, *)$ and $(n, *)$ - of the [LGP-Gaussian] log-theta-lattice to one another,

it is necessary to relate these unit and value group portions to one another by means of a single $\Theta_{\mathrm{LGP}}^{\times \boldsymbol{\mu}}$-link, i.e., from $(n-1, m)$ to $(n, m)$.

That is to say, from the point of view of constructing the various LGP-monoids that appear in the multiradial representation of Theorem 3.11, (i), one is tempted to work with correspondences between value groups on adjacent vertical lines that lie in a vertically once-shifted position - i.e., say, at $(n-1, m)$ and $(n, m)-$ relative to the correspondence between unit groups on adjacent vertical lines, i.e., say, at $(n-1, m-1)$ and $(n, m-1)$. On the other hand, such an approach fails, at least from an a priori point of view, precisely on account of the noncommutativity discussed in (i). Finally, we observe that in order to relate both unit and value groups by means of a single $\Theta_{\mathrm{LGP}}^{\times \boldsymbol{\mu}}-$ link,

it is necessary to avail oneself of the $\Theta_{\text {LGP }}^{\times \mu}$-link compatibility properties discussed in Theorem 3.11, (iii) - i.e., of the theory of $\S 2$ and [IUTchI], Example 5.1, (v) - so as to insulate the cyclotomes that appear in the construction of the étale theta function via mono-theta environments and the construction of number fields via global Kummer

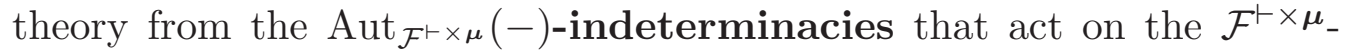
prime-strips involved as a result of the application of the $\Theta_{\mathrm{LGP}}^{\times \boldsymbol{\mu}}-\operatorname{link}$

— cf. the discussion of Remarks 2.2.1; 3.10.1, (ii).

(iii) As discussed in (ii) above, a "vertically once-shifted" approach to relating units on adjacent vertical lines fails on account of the noncommutativity discussed in 
(i). Thus, one natural approach to treating the units in a "vertically once-shifted" fashion - which, we recall, is necessary in order to relate the LGP-monoids on adjacent vertical lines to one another! — is to apply the bi-coricity of monoanalytic log-shells discussed in (i). On the other hand, to take this approach means that one must work in a framework that allows one to relate [cf. the discussion of Remark 1.5.4, (i)] the "Frobenius-like" structure constituted by the Frobenioidtheoretic units [i.e., which occur in the domain and codomain of the $\Theta_{\mathrm{LGP}}^{\times \mu}$-link] to corresponding étale-like structures simultaneously via both

(a) the usual Kummer isomorphisms - i.e., so as to be compatible with the application of the compatibility properties of Theorem 3.11, (iii), as discussed in (ii) — and

(b) the composite of the usual Kummer isomorphisms with [a single iterate of] the log-link - i.e., so as to be compatible with the bi-coric treatment of mono-analytic log-shells [as well as the closely related construction of LGP-monoids] proposed above.

Such a framework may only be realized if one relates Frobenius-like structures to étale-like structures in a fashion that is invariant with respect to pre-composition of various iterates of the log-link [cf. the final portions of Propositions 3.5, (ii); 3.10 , (ii)]. This is precisely what is achieved by the $\mathfrak{l o g}-$ Kummer correspondences of the final portion of Theorem 3.11, (ii).

(iv) The discussion of (i), (ii), (iii) above may be summarized as follows: The log-Kummer correspondences of the final portion of Theorem 3.11, (ii), allow one to

(a) relate both the unit and the value group portions of the domain and codomain of the $\Theta_{\mathrm{LGP}}^{\times \boldsymbol{\mu}}$-link corresponding to adjacent vertical lines of the [LGP-Gaussian] log-theta-lattice to one another, in a fashion that

(b) insulates the cyclotomes/Kummer theory surrounding the étale theta function and number fields involved from the $\operatorname{Aut}_{\mathcal{F} \vdash \times \mu}(-)$ indeterminacies that act on $\mathcal{F}^{\vdash \times \boldsymbol{\mu}}$-prime-strips involved as a result of the application of the $\Theta_{\mathrm{LGP}}^{\times \boldsymbol{\mu}}$-link [cf. Theorem 3.11, (iii)], and, moreover,

(c) is compatible with the bi-coricity of the mono-analytic log-shells [cf. Theorem 1.5, (iv)], hence also with the operation of relating the LGP-monoids that appear in the multiradial representation of Theorem 3.11, (i), corresponding to adjacent vertical lines of the [LGP-Gaussian] log-theta-lattice to one another.

These observations will play a key role in the proof of Corollary 3.12 below.

The following result may be thought of as a relatively concrete consequence of the somewhat abstract content of Theorem 3.11.

Corollary 3.12. (Log-volume Estimates for $\Theta$-Pilot Objects) Suppose that we are in the situation of Theorem 3.11. Write

$$
-|\log (\underline{\underline{\Theta}})| \in \mathbb{R} \bigcup\{+\infty\}
$$


for the procession-normalized mono-analytic log-volume [i.e., where the average is taken over $j \in \mathbb{F}_{l}^{*}-c f$. Remark 3.1.1, (ii); Proposition 3.9, (i), (ii); Theorem 3.11, (i), (a)] of the union of the possible images of a $\Theta$-pilot object [cf. Definition 3.8, (i)], relative to the relevant Kummer isomorphisms [cf. Theorem 3.11, (ii)], in the multiradial representation of Theorem 3.11, (i), which we regard as subject to the indeterminacies (Ind1), (Ind2), (Ind3) described in Theorem 3.11, (i), (ii). Write

$$
-|\log (\underline{\underline{q}})| \in \mathbb{R}
$$

for the procession-normalized mono-analytic log-volume of the image of a $\boldsymbol{q}$-pilot object [cf. Definition 3.8, (i)], relative to the relevant Kummer isomorphisms [cf. Theorem 3.11, (ii)], in the multiradial representation of Theorem 3.11, (i), which we do not regard as subject to the indeterminacies (Ind1), (Ind2), (Ind3) described in Theorem 3.11, (i), (ii). Here, we recall the definition of the symbol " $\triangle$ " as the result of identifying the labels

$$
\text { "0" and " }\left\langle\mathbb{F}_{l}^{*}\right\rangle "
$$

[cf. [IUTchII], Corollary 4.10, (i)]. In particular, $|\log (\underline{\underline{q}})|>0$ is easily computed in terms of the various $\boldsymbol{q}$-parameters of the elliptic curve $E_{F}$ [cf. [IUTchI], Definition 3.1, (b)] at $\underline{v} \in \underline{\mathbb{V}}^{\text {bad }}(\neq \emptyset)$. Then it holds that

$$
C_{\Theta} \geq-1
$$

for any real number $C_{\Theta} \in \mathbb{R}$ such that

$$
-|\log (\underline{\underline{\Theta}})| \leq C_{\Theta} \cdot|\log (\underline{\underline{q}})|
$$

[i.e., $-|\log (\underline{\underline{\Theta}})| \in \mathbb{R} \subseteq \mathbb{R} \bigcup\{+\infty\}$ and satisfies the inequality $\left.\left({ }^{*} C_{\Theta}\right)\right]$.

Proof. Suppose that we are in the situation of Theorem 3.11. We begin by reviewing precisely what is achieved by the various portions of Theorem 3.11 and, indeed, by the theory developed thus far in the present series of papers. This review leads naturally to an interpretation of the theory that gives rise to the inequality asserted in the statement of Corollary 3.12. For ease of reference, we divide our discussion into steps, as follows.

(i) In the following discussion, we concentrate on a single arrow - i.e., a single $\Theta_{\mathrm{LGP}}^{\times \boldsymbol{\mu}}-\operatorname{link}$

$$
{ }^{0,0} \mathcal{H} \mathcal{T}^{\Theta^{ \pm \mathrm{ell}} \mathrm{NF}} \stackrel{\Theta_{\mathrm{LGP}}^{\times \mu}}{\longrightarrow} \quad 1,0 \mathcal{H} \mathcal{T}^{\Theta^{ \pm \mathrm{ell}} \mathrm{NF}}
$$

— of the [LGP-Gaussian] log-theta-lattice under consideration. This arrow consists of the full poly-isomorphism of $\mathcal{F}^{\Vdash} \triangleright \times \boldsymbol{\mu}_{\text {-prime-strips }}$

$$
{ }_{0,0} \mathfrak{F}_{\mathrm{LGP}}^{\mapsto \sim} \stackrel{\sim}{\rightarrow}{ }^{1,0} \mathfrak{F}_{\triangle}^{\Vdash \vdash} \times \boldsymbol{\mu}
$$

[cf. Definition 3.8, (ii)]. This poly-isomorphism may be thought of as consisting of a "unit portion" constituted by the associated [full] poly-isomorphism of $\mathcal{F}^{-\times \boldsymbol{\mu}_{-}}$ prime-strips

$$
0,0 \mathfrak{F}_{\mathrm{LGP}}^{\vdash \times \mu} \stackrel{\sim}{\rightarrow}{ }^{1,0} \mathfrak{F}_{\triangle}^{\vdash \times \mu}
$$


and a "value group portion" constituted by the associated [full] poly-isomorphism of $\mathcal{F}^{\Vdash \vdash}$-prime-strips

$$
{ }^{0,0} \mathfrak{F}_{\mathrm{LGP}}^{\stackrel{\leftrightarrow}{\longrightarrow}} \stackrel{\sim}{\rightarrow}{ }^{1,0} \mathfrak{F}_{\triangle}
$$

[cf. Definition 2.4, (iii)]. This value group portion of the $\Theta_{\mathrm{LGP}}^{\times \mu}$-link maps $\Theta$-pilot objects of ${ }^{0,0} \mathcal{H} \mathcal{T}^{\Theta^{ \pm \text {ell }} \mathrm{NF}}$ to q-pilot objects of ${ }^{1,0} \mathcal{H} \mathcal{T}^{\Theta^{ \pm \text {ell }} \mathrm{NF}}$ [cf. Remark 3.8.1].

(ii) Whereas the units of the Frobenioids that appear in the $\mathcal{F}^{\vdash \times \boldsymbol{\mu}}$-prime-strip $0,0 \mathfrak{F}_{\text {LGP }}^{\vdash \times \mu}$ are subject to $\operatorname{Aut}_{\mathcal{F} \vdash \times \mu}(-)$-indeterminacies [i.e., "(Ind2)" — cf. Theorem 3.11, (iii), (a), (b)], the cyclotomes that appear in the Kummer theory related to the étale theta function and the number fields involved, i.e., which give rise to the "value group portion" ${ }^{0,0} \mathfrak{F}_{\mathrm{LGP}}^{\vdash}$, are insulated from these $\operatorname{Aut}_{\mathcal{F}^{\vdash} \times \mu}(-)-$ indeterminacies - cf. Theorem 3.11, (iii), (c), (d); the discussion of Remark 3.11.3, (iv); Fig. 3.4 below. Here, we recall that in the case of the étale theta function, this follows from the theory of $\S 2$, i.e., in essence, from the cyclotomic rigidity of mono-theta environments, as discussed in [EtTh]. On the other hand, in the case of number fields, this follows, in essence, from the elementary algebraic number theory fact applied in the proof of Proposition 3.10 to the effect that the nonzero global integers of a number field are necessarily roots of unity, together with the algorithms discussed in [IUTchI], Example 5.1, (v).

\begin{tabular}{|c|c|c|}
\hline & $\underline{\Theta}$-related objects & $\underline{N F-\text { related objects }}$ \\
\hline $\begin{array}{c}\text { require } \\
\text { mono-analytic } \\
\text { containers, } \\
\text { Kummer-incompatible }\end{array}$ & $\begin{array}{l}\text { local LGP-monoids } \\
\begin{array}{c}\text { [cf. Proposition } \\
\text { 3.4, (ii) }]\end{array}\end{array}$ & $\begin{array}{l}\quad F_{\text {mod }} \\
{[\text { cf. Proposition }} \\
\text { 3.7, (i)] }\end{array}$ \\
\hline $\begin{array}{c}\text { independent of } \\
\text { mono-analytic } \\
\text { containers, } \\
\text { Kummer-compatible }\end{array}$ & $\begin{array}{c}\text { étale theta } \\
\text { function, } \\
\text { mono-theta } \\
\text { environments } \\
\text { [cf. Corollary 2.3] }\end{array}$ & $\begin{array}{c}\bar{F} \\
\text { [cf. [IUTchI], } \\
\text { Example 5.1, (v); } \\
\text { [IUTchII], } \\
\text { Corollary 4.8, (i)] }\end{array}$ \\
\hline
\end{tabular}

Fig. 3.4: Relationship of theta- and number field-related objects to mono-analytic containers

(iii) In the following discussion, it will be of crucial importance to relate simultaneously both the unit and the value group portions of the $\Theta_{\mathrm{LGP}}^{\times \boldsymbol{\mu}}-\operatorname{link}(\mathrm{s})$ involved on the 0-column [i.e., the vertical line indexed by 0] of the log-theta-lattice under consideration to the corresponding unit and value group portions on the 1-column [i.e., the vertical line indexed by 1] of the log-theta-lattice under consideration. On the other hand, if one attempts to relate the unit portions via 
one $\Theta_{\mathrm{LGP}}^{\times \boldsymbol{\mu}}$-link $[$ say, from $(0, m)$ to $(1, m)]$ and the value group portions via another $\Theta_{\mathrm{LGP}}^{\times \boldsymbol{\mu}}$-link [say, from $\left(0, m^{\prime}\right)$ to $\left(1, m^{\prime}\right)$, for $m^{\prime} \neq m$ ], then the non-commutativity of the log-theta-lattice renders it practically impossible to obtain conclusions that require one to relate both the unit and the value group portions simultaneously [cf. the discussion of Remark 3.11.3, (i), (ii)]. This is precisely why we concentrate on a single $\Theta_{\mathrm{LGP}}^{\times \boldsymbol{\mu}}$-link [cf. (i)].

(iv) The issue discussed in (iii) is relevant in the context of the present discussion for the following reason. Ultimately, we wish to apply the bi-coricity of the units [cf. Theorem 1.5, (iii), (iv)] in order to compute the 0-column $\Theta$-pilot object in terms of the arithmetic holomorphic structure of the 1-column. In order to do this, one must work with units that are vertically once-shifted [i.e., lie at $(n, m-1)]$ relative to the value group structures involved [i.e., which lie at $(n, m)]$ — cf. the discussion of Remark 3.11.3, (ii). The solution to the problem of simultaneous accommodating these apparently contradictory requirements - i.e., "vertical shift" vs. "impossibility of vertical shift" [cf. (iii)] — is given precisely by working, on the 0 -column, with structures that are invariant with respect to vertical shifts [i.e., " $(0, m) \mapsto(0, m+1)$ "] of the log-theta-lattice [cf. the discussion surrounding Remark 1.2.2, (iii), (a)] such as vertically coric structures [i.e., indexed by " $(n, \circ)$ "] that are related to the "Frobenius-like" structures which are not vertically coric by means of the log-Kummer correspondences of Theorem 3.11, (ii). Here, we note that this "solution" may be implemented only at the cost of admitting the "indeterminacy" constituted by the upper semi-compatibility of (Ind3).

(v) Thus, we begin our computation of the 0-column $\Theta$-pilot object in terms of the arithmetic holomorphic structure of the 1-column by relating the units on the 0 - and 1-columns by means of the unit portion

$$
{ }^{0,0} \mathfrak{F}_{\mathrm{LGP}}^{\vdash \times \mu} \stackrel{\sim}{\rightarrow} 1,0 \mathfrak{F}_{\triangle}^{\vdash \times \mu}
$$

of the $\Theta_{\mathrm{LGP}}^{\times \boldsymbol{\mu}}$-link from $(0,0)$ to $(1,0)[\mathrm{cf} .(\mathrm{i})]$ and then applying the bi-coricity of the units of Theorem 1.5, (iii), (iv). In particular, the mono-analytic log-shell interpretation of this bi-coricity given in Theorem 1.5, (iv), will be applied to regard these mono-analytic log-shells as "multiradial mono-analytic containers" [cf. the discussion of Remark 1.5.2, (i), (ii), (iii)] for the various [local and global] value group structures that constitute the $\Theta$-pilot object on the 0 -column - cf. Fig. 3.4 above. [Here, we observe that the parallel treatment of "theta-related" and "number field-related" objects is reminiscent of the discussion of [IUTchII], Remark 4.11.2, (iv).] That is to say, we will relate the various Frobenioid-theoretic [i.e., "Frobenius-like" — cf. Remark 1.5.4, (i)]

- local units at $\underline{v} \in \underline{\mathbb{V}}$,

- splitting monoids at $\underline{v} \in \underline{\mathbb{V}}^{\text {bad }}$, and

- global Frobenioids

indexed by $(0, m)$, for $m \in \mathbb{Z}$, to the vertically coric [i.e., indexed by "(0, o)"] versions of these bi-coric mono-analytic containers by means of the log-Kummer 
correspondences of Theorem 3.11, (ii), (a), (b), (c) - i.e., by varying the "Kummer input index" $(0, m)$ along the 0 -column.

(vi) In the context of (v), it is useful to recall that the log-Kummer correspondences of Theorem 3.11, (ii), (b), (c), are obtained precisely as a consequence of the splittings, up to roots of unity, of the relevant monoids into unit and value group portions constructed by applying the constant multiple rigidity established in [EtTh] [in the case of Theorem 3.11, (ii), (b)] and the elementary algebraic number theory characterization of the global integers of a number field that was applied in the proof of Proposition 3.10 [in the case of Theorem 3.11, (ii), (c)]. Moreover, we recall that the Kummer theory surrounding the local LGP-monoids of Proposition 3.4, (ii), depends, in an essential way, on the theory of [IUTchII], $\S 3$ [cf., especially, [IUTchII], Corollaries 3.5, 3.6], which, in turn, depends, in an essential way, on the Kummer theory surrounding mono-theta environments established in [EtTh]. Thus, for instance, we recall that the discrete rigidity established in [EtTh] is applied so as to avoid working, in the tempered Frobenioids

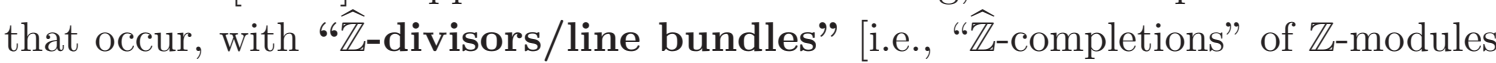
of divisors/line bundles], which are fundamentally incompatible with conventional notions of divisors/line bundles, hence, in particular, with mono-theta-theoretic cyclotomic rigidity [cf. Remark 2.1.1, (v)]. Also, we recall that "isomorphism class compatibility" - i.e., in the terminology of [EtTh], "compatibility with the topology of the tempered fundamental group" [cf. the discussion at the beginning of Remark 2.1.1] — allows one to apply the Kummer theory of mono-theta environments [i.e., the theory of [EtTh]] relative to the ring-theoretic basepoints that occur on either side of the log-link [cf. Remark 2.1.1, (ii); [IUTchII], Remark 3.6.4, (i)], for instance, in the context of the log-Kummer correspondence for the splitting monoids of local LGP-monoids, whose construction depends, in an essential way [cf. the theory of [IUTchII], §3, especially, [IUTchII], Corollaries 3.5, 3.6], on the conjugate synchronization arising from the $\mathbb{F}_{l}^{\rtimes \pm}$-symmetry. That is to say,

it is precisely by establishing this conjugate synchronization arising from the $\mathbb{F}_{l}^{\rtimes \pm}$-symmetry relative to these basepoints that occur on either side of the log-link that one is able to conclude the crucial compatibility of this conjugate synchronization with the log-link discussed in Remark 1.3.2.

A similar observation may be made concerning the MLF-Galois pair approach to the cyclotomic rigidity isomorphism that is applied at $\underline{v} \in \underline{\mathbb{V}}^{\text {good }} \cap \underline{\mathbb{V}}^{\text {non }}[$ cf. [IUTchII], Corollary 1.11, (a); [IUTchII], Remark 1.11.1, (i), (a); [IUTchII], Proposition 4.2, (i); [AbsTopIII], Proposition 3.2, (iv)], which amounts, in essence, to

computations involving the Galois cohomology groups of various subquotients — such as torsion subgroups [i.e., roots of unity] and associated value groups - of the [multiplicative] module of nonzero elements of an algebraic closure of the mixed characteristic local field involved

[cf. the proof of [AbsAnab], Proposition 1.2.1, (vii)] - i.e., algorithms that are compatible with the topology of the profinite groups involved, in the sense that they do not require one to pass to Kummer towers [cf. the discussion of [IUTchII], 
Remark 3.6.4, (i)], which have are fundamentally incompatible with the ring structure of the fields involved. Here, we note in passing that the corresponding property for $\underline{v} \in \underline{\mathbb{V}}^{\text {arc }}$ [cf. [IUTchII], Proposition 4.4, (i)] holds for the simple reason that the complex archimedean local fields that occur at such $\underline{v}$ are already algebraically closed [so that there is no issue of possibly having to pass to Kummer towers]! On the other hand, the approaches to cyclotomic rigidity just discussed for $\underline{v} \in \underline{\mathbb{V}}^{\text {bad }}$ and $\underline{v} \in \underline{\mathbb{V}}^{\text {good }} \cap \underline{\mathbb{V}}^{\text {non }}$ differ quite fundamentally from the approach to cyclotomic rigidity taken in the case of [global] number fields in the algorithms described in [IUTchI], Example 5.1, (v), which depend, in an essential way, on the property

$$
\mathbb{Q}_{>0} \bigcap \widehat{\mathbb{Z}}^{\times}=\{1\}
$$

- i.e., which is fundamentally incompatible with the topology of the profinite groups involved in the sense that it clearly cannot be obtained as some sort of limit of corresponding properties of $(\mathbb{Z} / N \mathbb{Z})^{\times}$! Nevertheless, with regard to uni-/multiradiality issues, this approach to cyclotomic rigidity in the case of the number fields resembles the theory of mono-theta-theoretic cyclotomic rigidity at $\underline{v} \in \underline{\mathbb{V}}^{\text {bad }}$ in that it admits a natural multiradial formulation [cf. Theorem 3.11, (iii), (c), (d); the discussion of Remark 3.11.3], in sharp contrast to the essentially uniradial nature of the approach to cyclotomic rigidity via MLF-Galois pairs at $\underline{v} \in \underline{\mathbb{V}}^{\text {good }} \cap \underline{\mathbb{V}}^{\text {non }}[\mathrm{cf}$. the discussion of [IUTchII], Remark 1.11.3]. These observations are summarized in Fig. 3.5 below. Finally, we recall that [one verifies immediately that] the various approaches to cyclotomic rigidity just discussed are mutually compatible in the sense that they yield the same cyclotomic rigidity isomorphism in any setting in which more than one of these approaches may be applied.

\begin{tabular}{|c|c|c|}
\hline$\frac{\frac{\text { Approach to }}{\text { cyclotomic }}}{\text { rigidity }}$ & $\frac{\text { Uni-/multi- }}{\text { radiality }}$ & $\begin{array}{c}\text { Compatibility with ring } \\
\text { structure, log-link in the } \\
\text { context of the } \mathbb{F}_{l}^{\rtimes \pm} \text {-symmetry }\end{array}$ \\
\hline $\begin{array}{l}\text { mono-theta } \\
\text { environments }\end{array}$ & multiradial & compatible \\
\hline $\begin{array}{l}\text { MLF-Galois pairs, } \\
\text { via Brauer groups }\end{array}$ & uniradial & compatible \\
\hline $\begin{array}{l}\text { number fields, via } \\
\mathbb{Q}_{>0} \cap \widehat{\mathbb{Z}}^{\times}=\{1\}\end{array}$ & $\begin{array}{c}\text { multiradial } \\
(\Longleftarrow \exists \text { splitting })\end{array}$ & incompatible \\
\hline
\end{tabular}

Fig. 3.5: Three approaches to cyclotomic rigidity

(vii) In the context of the discussion in the final portion of (vi), it is of interest to recall that the constructions underlying the crucial bi-coricity theory of 
Theorem 1.5, (iii), (iv), depend, in an essential way, on the conjugate synchronization arising from the $\mathbb{F}_{l}^{\rtimes \pm}$-symmetry, which allows one to relate the local monoids and Galois groups at distinct labels $\in\left|\mathbb{F}_{l}\right|$ to one another in a fashion that is simultaneously compatible both with

- the vertically coric structures and Kummer theory that give rise to the log-Kummer correspondences of Theorem 3.11, (ii),

and with

- the property of distinguishing [i.e., not identifying] data indexed by distinct labels $\in\left|\mathbb{F}_{l}\right|$

- cf. the discussion of Remark 1.5.1, (i), (ii). Since, moreover, this crucial conjugate synchronization is fundamentally incompatible with the $\mathbb{F}_{l}^{*}$-symmetry, it is necessary to work with these two symmetries separately, as was done in [IUTchI], $\S 4, \S 5, \S 6$ [cf. [IUTchII], Remark 4.7.6]. Here, it is useful to recall that the $\mathbb{F}_{i}^{*}$ symmetry also plays a crucial role, in that it allows one to "descend to $F_{\bmod }$ " at the level of absolute Galois groups [cf. [IUTchII], Remark 4.7.6]. On the other hand, both the $\mathbb{F}_{l}^{\rtimes \pm}$ - and $\mathbb{F}_{l}^{*}$-symmetries share the property of being compatible with the vertical coricity and relevant Kummer isomorphisms of the 0-column - cf. the log-Kummer correspondences of Theorem 3.11, (ii), (b) [in the case of the $\mathbb{F}_{l}^{\rtimes \pm}$-symmetry], (c) [in the case of the $\mathbb{F}_{l}^{*}$-symmetry]. Indeed, the vertically coric versions of both the $\mathbb{F}_{l}^{\rtimes \pm}$ - and the $\mathbb{F}_{l}^{*}$-symmetries depend, in an essential way, on the arithmetic holomorphic structure of the 0-column, hence give rise to multiradial structures via the tautological approach to constructing such structures discussed in Remark 3.11.2, (i), (ii).

(viii) In the context of (vii), it is useful to recall that in order to construct the $\mathbb{F}_{l}^{\rtimes \pm}$-symmetry, it is necessary to make use of global \pm -synchronizations of various local \pm -indeterminacies. Since the local tempered fundamental groups at $\underline{v} \in \underline{\mathbb{V}}^{\text {bad }}$ do not extend to a "global tempered fundamental group", these global \pm synchronizations give rise to profinite conjugacy indeterminacies in the vertically coric construction of the LGP-monoids [i.e., the theta values at torsion points] given in [IUTchII], $\S 2$, which are resolved by applying the theory of [IUTchI], $\S 2-$ cf. the discussion of [IUTchI], Remark 6.12.4, (iii); [IUTchII], Remark 4.5.3, (iii); [IUTchII], Remark 4.11.2, (iii).

(ix) In the context of (vii), it is also useful to recall the important role played, in the theory of the present series of papers, by the various "copies of $F_{\text {mod" }}$ ", i.e., more concretely, in the form of the various copies of the global Frobenioids " $\mathcal{F}_{\text {MOD }}^{\odot}$ ", " $\mathcal{F}_{\mathfrak{m o d}}^{\odot}$ " and their realifications. That is to say, the ring structure of the global field $F_{\text {mod }}$ allows one to bridge the gap — i.e., furnishes a translation apparatus - between the multiplicative structures constituted by the global realified Frobenioids related via the $\Theta_{\mathrm{LGP}}^{\times \boldsymbol{\mu}}$-link and the additive representations of these global Frobenioids that arise from the "mono-analytic containers" furnished by the mono-analytic log-shells [cf. (v)]. Here, the precise compatibility of the ingredients for " $\mathcal{F}_{\text {MOD }}^{\odot}$ " with the log-Kummer correspondence renders " $\mathcal{F}_{\text {MOD }}^{\odot}$ " better suited to describing the relation to the $\Theta_{\mathrm{LGP}}^{\times \boldsymbol{\mu}}$-link [cf. Remark 3.10.2, (ii)]. 
On the other hand, the local portion of " $\mathcal{F}_{\mathfrak{m o d}}^{\odot}$ " - i.e., which is subject to "upper semi-compatibility" [cf. (Ind3)], hence only "approximately compatible" with the log-Kummer correspondence - renders it better suited to explicit estimates of global arithmetic degrees, by means of log-volumes [cf. Remark 3.10.2, (iii)].

(x) Thus, one may summarize the discussion thus far as follows. The theory of "Kummer-detachment" — cf. Remarks 1.5.4, (i); 2.1.1; 3.10.2, (ii), (iii) — furnished by Theorem 3.11, (ii), (iii), allows one to relate the Frobenoid-theoretic [i.e., "Frobenius-like"] structures that appear in the domain [i.e., at $(0,0)$ ] of the $\Theta_{\text {LGP }}^{\times \boldsymbol{\mu}}$-link [cf. (i)] to the multiradial representation described in Theorem 3.11 , (i), (a), (b), (c), but only at the cost of introducing the indeterminacies

(Ind1) - which may be thought of as arising from the requirement of compatibility with the permutation symmetries of the étale-picture [cf. Theorem 3.11, (i)];

(Ind2) — which may be thought of as arising from the requirement of compatibility with the $\operatorname{Aut}_{\mathcal{F} \vdash \times \mu}(-)$-indeterminacies that act on the domain/codomain of the $\Theta_{\mathrm{LGP}}^{\times \boldsymbol{\mu}}$-link [cf. (ii); Theorem 3.11, (i), (iii)], i.e., with the horizontal arrows of the log-theta-lattice;

(Ind3) — which may be thought of as arising from the requirement of compatibility with the log-Kummer correspondences of Theorem 3.11, (ii), i.e., with the vertical arrows of the log-theta-lattice.

The various indeterminacies (Ind1), (Ind2), (Ind3) to which the multiradial representation is subject may be thought of as data that describes some sort of "formal quotient", like the "fine moduli spaces" that appear in algebraic geometry. In this context, the procession-normalized mono-analytic log-volumes [i.e., where the average is taken over $j \in \mathbb{F}_{l}^{*}$ ] of Theorem 3.11, (i), (a), (c), furnish a means of constructing a sort of associated "coarse space" or "inductive limit" [of the "inductive system" constituted by this "formal quotient"] — i.e., in the sense that [one verifies immediately - cf. Proposition 3.9, (ii) - that] the resulting $\log$-volumes $\in \mathbb{R}$ are invariant with to respect to the indeterminacies (Ind1), (Ind2), and have the effect of converting the indeterminacy (Ind3) into an inequality (from above). Moreover, the log-link compatibility of the various logvolumes that appear [cf. Proposition 3.9, (iv); the final portion of Theorem 3.11, (ii)] ensures that these log-volumes are compatible with [the portion of the "formal quotient" / "inductive system" constituted by] the various arrows [i.e., Kummer isomorphisms and $\mathfrak{l o g}$-links] of the log-Kummer correspondence of Theorem 3.11, (ii). Here, we note that the averages over $j \in \mathbb{F}_{l}^{*}$ that appear in the definition of the procession-normalized volumes involved may be thought of as a consequence of the $\mathbb{F}_{l}^{*}$-symmetry acting on the labels of the theta values that give rise to the LGP-monoids - cf. the also the definition of the symbol " $\triangle$ " in [IUTchII], Corollary 4.10, (i), via the identification of the symbols " 0 " and " $\left\langle\mathbb{F}_{l}^{*}\right\rangle$ "; the discussion of Remark 3.9.3. Also, in this context, it is of interest to observe that the various tensor products that appear in the various local mono-analytic tensor packets that arise in the multiradial representation of Theorem 3.11, (i), (a), have the effect of identifying the operation of "multiplication by elements of $\mathbb{Z}$ " — and hence also 
the effect on log-volumes of such multiplication operations! - at different labels $\in \mathbb{F}_{l}^{*}$.

(xi) Now let us consider a $\boldsymbol{q}$-pilot object at $(1,0)$, which we think of relative to the relevant copy of " $\mathcal{F}_{\mathfrak{m o d}}^{\odot}$ " - in terms of the mono-analytic logshells constructed at $(1,0)[\mathrm{cf} .(\mathrm{v})]$. Then the $\Theta_{\mathrm{LGP}}^{\times \boldsymbol{\mu}}$-link from $(0,0)$ to $(1,0)$ may be interpreted as a sort of gluing isomorphism that relates the arithmetic holomorphic structure - i.e., the "conventional ring/scheme-theory" - at $(1,0)$ to the arithmetic holomorphic structure at $(0,0)$ in such a way that the $\Theta$-pilot object at $(0,0)$ [thought of as an object of the relevant global realified Frobenioid] corresponds to the $\boldsymbol{q}$-pilot object at $(1,0)$ [cf. (i)]. On the other hand, the discussion of $(\mathrm{x})$ furnishes another way of computing the global arithmetic degree — i.e., the log-volume [cf. Theorem 3.11, (i), (c)] — of this q-pilot object at $(1,0)$, namely, by computing the log-volume of the $\Theta$-pilot object $(0,0)$, constructed relative to the alien [i.e., from the point of view of the arithmetic holomorphic structure at $(1,0)]$ arithmetic holomorphic structure at $(0,0)$, in terms of the arithmetic holomorphic structure at $(1,0)$ [cf. the final portion of Theorem 3.11, (i); Remark 3.11.1]. That is to say,

the theory of the present series of papers yields two tautologically equivalent ways to compute the log-volume of the q-pilot object at $(1,0)$
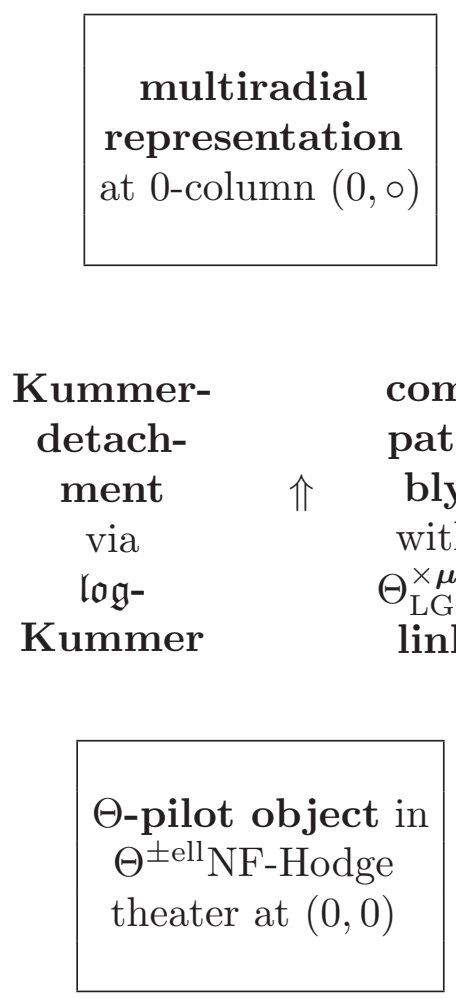

$$
\begin{gathered}
\text { permutation } \\
\text { symmetry of } \\
\approx \\
\text { étale-picture }
\end{gathered}
$$

multiradial representation at 1-column $(1, \circ)$

Fig. 3.6: Two tautologically equivalent ways to compute the log-volume of the $q$-pilot object at $(1,0)$

— cf. Fig. 3.6. If one interprets this observation in terms of the notation introduced in the statement of Corollary 3.12, then one concludes that

$$
-|\log (\underline{\underline{q}})| \leq-|\log (\underline{\underline{\Theta}})| \in \mathbb{R}
$$


— hence, in particular, that

$$
-|\log (\underline{\underline{q}})| \leq C_{\Theta} \cdot|\log (\underline{\underline{q}})|
$$

for any $C_{\Theta} \in \mathbb{R}$ such that $-|\log (\underline{\underline{\Theta}})| \leq C_{\Theta} \cdot|\log (\underline{\underline{q}})|$. Since [one verifies immediately that $|\log (\underline{q})| \in \mathbb{R}$ is positive, we thus conclude that $C_{\Theta} \geq-1$, as desired. In this context, it is useful to recall that the above argument depends, in an essential way [cf. the discussion of (ii), (vi)], on the theory of [EtTh], which does not admit any evident generalization to the case of $N$-th tensor powers of $\Theta$-pilot objects, for $N \geq 2$. That is to say, the log-volume of such an $N$-th tensor power of a $\Theta$-pilot object must always be computed as the result of multiplying the log-volume of the original $\Theta$-pilot object by $N$ - cf. Remark 2.1.1, (iv); [IUTchII], Remark 3.6.4, (iii), (iv). In particular, although the analogue of the above argument for such an $N$-th tensor power would lead to sharper inequalities than the inequalities obtained here, it is difficult to see how to obtain such sharper inequalities via a routine generalization of the above argument. In fact, as we shall see in [IUTchIV], these sharper inequalities are known to be false [cf. [IUTchIV], Remark 2.3.2, (ii)].

(xii) In the context of the argument of (xi), it is useful to observe the important role played by the global realified Frobenioids that appear in the $\Theta_{\mathrm{LGP}}^{\times \boldsymbol{\mu}}$-link. That is to say, since ultimately one is only concerned with the computation of log-volumes, it might appear, at first glance, that it is possible to dispense with the use of such global Frobenioids and instead work only with the various local Frobenioids, for $\underline{v} \in \mathbb{V}$, that are directly related to the computation of log-volumes. On the other hand, observe that since the isomorphism of [local or global!] Frobenioids arising from the $\Theta_{\mathrm{LGP}}^{\times \boldsymbol{\mu}}$-link only preserves isomorphism classes of objects of these Frobenioids [cf. the discussion of Remark 3.6.2, (i)], to work only with local Frobenioids means that one must contend with the indeterminacy of not knowing whether, for instance, such a local Frobenioid object at some $\underline{v} \in \mathbb{V}^{\text {non }}$ corresponds to a given open submodule of the log-shell at $\underline{v}$ or to, say, the $p_{v}^{N}$-multiple of this submodule, for $N \in \mathbb{Z}$. Put another way, one must contend with the indeterminacy arising from the fact that, unlike the case with the global Frobenioids " $\mathcal{F}_{\text {MOD }}^{\odot}$ ", " $\mathcal{F}_{\text {MOD }}^{\odot \mathbb{R}}$ ", objects of the various local Frobenioids that arise admit endomorphisms which are not automorphisms. This indeterminacy has the effect of rendering meaningless any attempt to perform a precise log-volume computation as in (xi).

\section{Remark 3.12.1.}

(i) In [IUTchIV], we shall be concerned with obtaining more explicit upper bounds on $-|\log (\underline{\underline{\Theta}})|$, i.e., estimates " $C_{\Theta}$ " as in the statement of Corollary 3.12.

(ii) It is not difficult to verify that, for $\lambda \in \mathbb{Q}_{>0}$, one may obtain a similar theory to the theory developed in the present series of papers for "generalized $\Theta_{\mathrm{LGP}}^{\times \boldsymbol{\mu}}$-links" of the form

$$
\underline{\underline{q}}^{\lambda} \mapsto \underline{\underline{q}}\left(\begin{array}{c}
1^{2} \\
\vdots \\
(l *)^{2}
\end{array}\right)
$$


- i.e., so the theory developed in the present series of papers corresponds to the case of $\lambda=1$. This sort of "generalized $\Theta_{\mathrm{LGP}}^{\times \mu}$-link" is roughly reminiscent of but by no means equivalent to! - the sort of issues considered in the discussion of Remark 2.2.2, (i). Here, we observe that raising to the $\lambda$-th power on the " $q$ side" differs quite fundamentally from raising to the $\lambda$-th power on the " $\underline{q}^{\left(1^{2} \ldots\left(l^{*}\right)^{2}\right)}$ side", an issue that is discussed briefly [in the case of $\lambda=N$ ] in the final portion of Step (xi) of the proof of Corollary 3.12. That is to say, "generalized $\Theta_{\text {LGP }}^{\times \boldsymbol{\mu}}$-links" as in the above display differ fundamentally both from the situation of Remark 2.2.2, (i), and the situation discussed in the final portion of Step (xi) of the proof of Corollary 3.12 in that the theory of the first power of the étale theta function is left unchanged [i.e., relative to the theory developed in the present series of papers] — cf. the discussion of Remark 2.2.2, (i); Step (xi) of the proof of Corollary 3.12. At any rate, in the case of "generalized $\Theta_{\mathrm{LGP}}^{\times \mu}-$ links" as in the above display, one may apply the same arguments as the arguments used to prove Corollary 3.12 to conclude the inequality

$$
C_{\Theta} \geq-\lambda
$$

- i.e., which is sharper, for $\lambda<1$, than the inequality obtained in Corollary 3.12 in the case of $\lambda=1$. In fact, however, such sharper inequalities will not be of interest to us, since, in [IUTchIV], our estimates for the upper bound $C_{\Theta}$ will be sufficiently rough as to be unaffected by adding a constant of absolute value $\leq 1$.

\section{Remark 3.12.2.}

(i) One of the main themes of the present series of papers is the issue of dismantling the two underlying combinatorial dimensions of a number field - cf. Remarks 1.2.2, (vi), of the present paper, as well as [IUTchI], Remarks 3.9.3, 6.12.3, 6.12.6; [IUTchII], Remarks 4.7.5, 4.7.6, 4.11.2, 4.11.3, 4.11.4. The principle examples of this topic may be summarized as follows.

(a) splittings of various monoids into unit and value group portions;

(b) separating the " $\mathbb{F}_{l}$ " arising from the $l$-torsion points of the elliptic curve - which may be thought of as a sort of "finite approximation" of $\mathbb{Z}$ ! into a [multiplicative] $\mathbb{F}_{l}^{*}$-symmetry — which may also be thought of as corresponding to the global arithmetic portion of the arithmetic fundamental groups involved — and a(n) [additive $\mathbb{F}_{l}^{\rtimes \pm}$-symmetry — which may also be thought of as corresponding to the geometric portion of the arithmetic fundamental groups involved;

(c) separating the ring structures of the various global number fields that appear into their respective underlying additive structures - which may be related directly to the various log-shells that appear — and their respective underlying multiplicative structures - which may be related directly to the various Frobenioids that appear.

From the point of view of Theorem 3.11, example (a) may be seen in the "noninterference" properties that underlie the log-Kummer correspondences of 
Theorem 3.11, (ii), (b), (c), as well as in the $\Theta_{\text {LGP }}^{\times \mu}$-link compatibility properties discussed in Theorem 3.11, (ii), (c), (d).

(ii) On the other hand, another important theme of the present $\S 3$ consists of the issue of "reassembling" these two dismantled combinatorial dimensions by means of the multiradial mono-analytic containers furnished by the monoanalytic log-shells - cf. Fig. 3.4 - i.e., of exhibiting the extent to which these two dismantled combinatorial dimensions cannot be separated from one another by describing the "structure of the intertwining" between these two dimensions that existed prior to their separation. From this point of view, one may think of the multiradial representations discussed in Theorem 3.11, (i) [cf. also Theorem 3.11, (ii), (iii)], as the final output of this "reassembling procedure". Put another way, from the point of view of example (a) of the discussion of (i), this "reassembling procedure" allows one to compute/estimate the value group portions of various monoids of arithmetic interest in terms of the unit group portions of these monoids. It is precisely these estimates that give rise to the inequality obtained in Corollary 3.12.

(iii) One fundamental aspect of the theory that renders possible the "reassembling procedure" discussed in (ii) [cf. the discussion of Step (iv) of the proof of Corollary 3.12] is the "juggling of $\boxplus,{ }^{\prime}$ " [cf. the discussion of Remark 1.2.2, (vi)] effected by the log-links, i.e., the vertical arrows of the log-theta-lattice. This "juggling of $\boxplus, \otimes "$ may be thought of as a sort of combinatorial way of representing the arithmetic holomorphic structure associated to a vertical line of the log-theta-lattice. Indeed, at archimedean primes, this juggling amounts essentially to multiplication by $\pm i$, which is a well-known method [cf. the notion of an "almost complex structure"!] for representing holomorphic structures in the classical theory of differential manifolds. On the other hand, it is important to recall in this context that this "juggling of $\boxplus, \otimes$ " is precisely what gives rise to the upper semi-compatibility indeterminacy (Ind3) [cf. Proposition 3.5, (ii); Remark 3.10.2, (i)].

\section{Remark 3.12.3.}

(i) Let $S$ be a hyperbolic Riemann surface of finite type of genus $g_{S}$ with $r_{S}$ punctures. Write $\chi_{S} \stackrel{\text { def }}{=}-\left(2 g_{S}-2+r_{S}\right)$ for the Euler characteristic of $S$ and $d \mu_{S}$ for the Kähler metric on $S$ [i.e., the $(1,1)$-form] determined by the Poincaré metric on the upper half-plane. Recall the analogy discussed in [IUTchI], Remark 4.3.3, between the theory of log-shells, which plays a key role in the theory developed in the present series of papers, and the classical metric geometry of hyperbolic Riemann surfaces. Then, relative to this analogy, the inequality obtained in Corollary 3.12 may be regarded as corresponding to the inequality

$$
\chi_{S}=-\int_{S} d \mu_{S}<0
$$

- i.e., in essence, a statement of the hyperbolicity of $S$ - arising from the classical Gauss-Bonnet formula, together with the positivity of $d \mu_{S}$. Relative to the analogy between real analytic Kähler metrics and ordinary Frobenius liftings 
discussed in $[p$ Ord], Introduction, $\S 2$ [cf. also the discussion of [pTeich], Introduction, §0], the local property constituted by this positivity of $d \mu_{S}$ may be thought of as corresponding to the [local property constituted by the] Kodaira-Spencer isomorphism of an indigenous bundle - i.e., which gives rise to the ordinarity of the corresponding Frobenius lifting on the ordinary locus — in the p-adic theory. As discussed in [AbsTopIII], §I5, these properties of indigenous bundles in the $p$-adic theory may be thought of as corresponding, in the theory of log-shells, to the "maximal incompatibility" between the various Kummer isomorphisms and the corically constructed data of the Frobenius-picture of Proposition 1.2, (x). On the other hand, it is just this "maximal incompatibility" that gives rise to the "upper semicommutativity" discussed in Remark 1.2.2, (iii), i.e., [from the point of view of the theory of the present $\S 3$ ] the upper semi-compatibility indeterminacy (Ind3) of Theorem 3.11, (ii), that underlies the inequality of Corollary 3.12 [cf. Step (x) of the proof of Corollary 3.12].

(ii) The "metric aspect" of Corollary 3.12 discussed in (i) is reminiscent of the analogy between the theory of the present series of papers and classical complex Teichmüller theory [cf. the discussion of [IUTchI], Remark 3.9.3] in the following sense:

Just as classical complex Teichmüller theory is concerned with relating distinct holomorphic structures in a sufficiently canonical way as to minimize the resulting volume distortion, the canonical nature of the algorithms discussed in Theorem 3.11 for relating alien arithmetic holomorphic structures [cf. Remark 3.11.1] gives rise to a relatively strong estimate of the [log-]volume distortion [cf. Corollary 3.12] resulting from such a deformation of the arithmetic holomorphic structure.

Remark 3.12.4. In light of the discussion of Remark 3.12.3, it is of interest to reconsider the analogy between the theory of the present series of papers and the $p$-adic Teichmüller theory of [ $p$ Ord], [ $p$ Teich], in the context of Theorem 3.11, Corollary 3.12.

(i) First, we observe that the splitting monoids at $\underline{v} \in \underline{\mathbb{V}}^{\text {bad }}[\mathrm{cf}$. Theorem 3.11, (i), (b); Theorem 3.11, (ii), (b)] may be regarded as analogous to the canonical coordinates of $p$-adic Teichmüller theory [cf., e.g., [ $p$ Teich], Introduction, §0.9] that are constructed over the ordinary locus of a canonical curve. In particular, it is natural to regard the bad primes $\in \mathbb{V}^{\text {bad }}$ as corresponding to the ordinary locus of a canonical curve and the good primes $\in \underline{\mathbb{V}}^{\text {good }}$ as corresponding to the supersingular locus of a canonical curve. This point of view is reminiscent of the discussion of [IUTchII], Remark 4.11.4, (iii).

(ii) On the other hand, the bi-coric mono-analytic log-shells - i.e., the various local " $\mathcal{O}^{\times \boldsymbol{\mu}}$ " — that appear in the tensor packets of Theorem 3.11, (i), (a); Theorem 3.11, (ii), (a), may be thought of as corresponding to the [multiplicative!] Teichmüller representatives associated to the various Witt rings that appear in p-adic Teichmüller theory. Within a fixed arithmetic holomorphic structure, these mono-analytic log-shells arise from "local holomorphic units" — i.e., " $\mathcal{O}^{\times}$" — which are subject to the $\mathbb{F}_{l}^{\rtimes \pm}$-symmetry. These "local holomorphic units" may be thought of as corresponding to the positive characteristic 
ring structures on [the positive characteristic reductions of] Teichmüller representatives. Here, the uniradial, i.e., "non-multiradial", nature of these "local holomorphic units" [cf. the discussion of [IUTchII], Remark 4.7.4, (ii); [IUTchII], Figs. 4.1, 4.2] may be regarded as corresponding to the mixed characteristic nature of Witt rings, i.e., the incompatibility of Teichmüller representatives with the additive structure of Witt rings.

(iii) The set $\mathbb{F}_{l}^{*}$ of $l *$ "theta value labels", which plays an important role in the theory of the present series of papers, may be thought of as corresponding to the "factor of $\boldsymbol{p}$ " that appears in the " $\bmod p / p^{2}$ portion", i.e., the gap separating the " $\bmod p$ " and " $\bmod p^{2} "$ portions, of the rings of Witt vectors that occur in the $p$-adic theory. From this point of view, one may think of the procession-normalized volumes obtained by taking averages over $j \in \mathbb{F}_{l}^{*}$ [cf. Corollary 3.12] as corresponding to the operation of dividing by $\boldsymbol{p}$ to relate the "mod $p / p^{2}$ portion" of the Witt vectors to the "mod $p$ portion" of the Witt vectors [i.e., the characteristic $p$ theory]. In this context, the multiradial representation of Theorem 3.11, (i), by means of mono-analytic log-shells labeled by elements of $\mathbb{F}_{l}^{*}$ may be thought of as corresponding to the derivative of the canonical Frobenius lifting on a canonical curve in the $p$-adic theory [cf. the discussion of [AbsTopIII], §I5] in the sense that this multiradial representation may be regarded as a sort of comparison of the canonical splitting monoids discussed in (i) to the "absolute constants" [cf. the discussion of (ii)] constituted by the bi-coric mono-analytic log-shells. This "absolute comparison" is precisely what results in the indeterminacies (Ind1), (Ind2) of Theorem 3.11, (i).

(iv) In the context of the discussion of (iii), we note that the set of labels $\mathbb{F}_{l}^{*}$ may, alternatively, be thought of as corresponding to the infinitesimal moduli of the positive characteristic curve under consideration in the $p$-adic theory [cf. the discussion of [IUTchII], Remark 4.11.4, (iii), (d)]. That is to say, the "deformation dimension" constituted by the horizontal dimension of the log-theta-lattice in the theory of the present series of papers or by the deformations modulo various powers of $p$ in the $p$-adic theory [cf. Remark 1.4.1, (iii); Fig. 1.3] is highly canonical in nature, hence may be thought of as being equipped with a natural isomorphism to the "absolute moduli" - i.e., so to speak, the "moduli over $\mathbb{F}_{1}$ " — of the given number field equipped with an elliptic curve, in the theory of the present series of papers, or of the given positive characteristic hyperbolic curve equipped with a nilpotent ordinary indigenous bundle, in p-adic Teichmüller theory.

(v) Let $A$ be a ring of Witt vectors of a perfect field $k$ of characteristic $p ; X$ a smooth, proper hyperbolic curve over $A$ of genus $g_{X}$ which is canonical in the sense of $p$-adic Teichmüller theory; $\widehat{X}$ the $p$-adic formal scheme associated to $X ; \widehat{U} \subseteq \widehat{X}$ the ordinary locus of $\widehat{X}$. Write $\omega_{X_{k}}$ for the canonical bundle of $X_{k}$. Then when [cf. the discussion of (iii)] one computes the derivative of the canonical Frobenius lifting $\Phi: \widehat{U} \rightarrow \widehat{U}$ on $\widehat{U}$, one must contend with "interference phenomena" between the various copies of some positive characteristic algebraic geometry set-up - i.e., at a more concrete level, the various Frobenius conjugates " $t^{n}$ " [where $t$ is a local coordinate on $\left.X_{k} \stackrel{\text { def }}{=} X \times_{A} k\right]$ associated to various $n \in \mathbb{N}_{\geq 1}$. In particular, this derivative only yields [upon dividing by $p$ ] an inclusion [i.e., not an isomorphism!] of line bundles

$$
\omega_{X_{k}} \hookrightarrow \Phi^{*} \omega_{X_{k}}
$$


— also known as the "[square] Hasse invariant" [cf. [ $p$ Ord], Chapter II, Proposition 2.6; the discussion of "generalities on ordinary Frobenius liftings" given in [ $p$ Ord], Chapter III, §1]. Thus, at the level of global degrees of line bundles, we obtain an inequality [i.e., not an equality!]

$$
(1-p)\left(2 g_{X}-2\right) \leq 0
$$

\begin{tabular}{|c|c|}
\hline inter-universal Teichmüller theory & p-adic Teichmüller theory \\
\hline $\begin{array}{l}\text { splitting monoids } \\
\qquad \text { at } \underline{v} \in \underline{\mathbb{V}}^{\text {bad }}\end{array}$ & $\begin{array}{l}\text { canonical coordinates } \\
\text { on the ordinary locus }\end{array}$ \\
\hline bad primes $\in \underline{\mathbb{V}}^{\text {bad }}$ & ordinary locus of a can. curve \\
\hline good primes $\in \underline{\mathbb{V}}^{\text {good }}$ & supersing. locus of a can. curve \\
\hline mono-analytic log-shells " $\mathcal{O}^{\times \mu} "$ & [multiplicative!] Teich. reps. \\
\hline $\begin{array}{l}\text { uniradial "local hol. units } \mathcal{O}^{\times "} \\
\text { subject to } \mathbb{F}_{l}^{\rtimes \pm} \text {-symmetry }\end{array}$ & $\begin{array}{l}\text { pos. char. ring structures on } \\
\text { [pos. char. reductions of] Teich. reps. }\end{array}$ \\
\hline $\begin{array}{l}\text { set of "theta value labels" } \\
\mathbb{F}_{i}^{*}\end{array}$ & $\begin{array}{c}\text { factor } \boldsymbol{p} \text { in } \\
\bmod p / p^{2} \text { portion of Witt vectors }\end{array}$ \\
\hline $\begin{array}{c}\text { multiradial rep. via } \mathbb{F}_{l}^{*} \text {-labeled } \\
\text { mono-analytic log-shells } \\
(\text { cf. }(\operatorname{Ind} 1),(\operatorname{Ind} 2))\end{array}$ & $\begin{array}{l}\text { derivative of the } \\
\text { canonical Frobenius lifting }\end{array}$ \\
\hline set of "theta value labels" $\mathbb{F}_{l}^{*}$ & implicit "absolute moduli/ $\mathbb{F}_{1}$ " \\
\hline $\begin{array}{l}\text { inequality arising from upper } \\
\text { semi-compatibility (cf. (Ind3)) }\end{array}$ & $\begin{array}{c}\text { inequality arising from interference } \\
\text { between Frobenius conjugates }\end{array}$ \\
\hline
\end{tabular}

Fig. 3.7: The analogy between inter-universal Teichmüller theory and $p$-adic Teichmüller theory 
- which may be thought of as being, in essence, a statement of the hyperbolicity of $X$ [cf. the inequality of the display of Remark 3.12.3, (i)]. Since the "Frobenius conjugate dimension" [i.e., the " $n$ " that appears in " $t^{p n "] ~ i n ~ t h e ~} p$-adic theory corresponds to the vertical dimension of the log-theta-lattice in the theory of the present series of papers [cf. Remark 1.4.1, (iii); Fig. 1.3], we thus see that the inequality of the above display in the $p$-adic case arises from circumstances that are entirely analogous to the circumstances - i.e., the upper semi-compatibility indeterminacy (Ind3) of Theorem 3.11, (ii) - that underlie the inequality of Corollary 3.12 [cf. Step (x) of the proof of Corollary 3.12; the discussion of Remark 3.12.3, (i)].

(vi) The analogies of the above discussion are summarized in Fig. 3.7 above. 


\section{Bibliography}

[Lang] S. Lang, Algebraic number theory, Addison-Wesley Publishing Co. (1970).

[pOrd] S. Mochizuki, A Theory of Ordinary p-adic Curves, Publ. Res. Inst. Math. Sci. 32 (1996), pp. 957-1151.

[pTeich] S. Mochizuki, Foundations of p-adic Teichmüller Theory, AMS/IP Studies in Advanced Mathematics 11, American Mathematical Society/International Press (1999).

[QuCnf] S. Mochizuki, Conformal and quasiconformal categorical representation of hyperbolic Riemann surfaces, Hiroshima Math. J. 36 (2006), pp. 405-441.

[SemiAnbd] S. Mochizuki, Semi-graphs of Anabelioids, Publ. Res. Inst. Math. Sci. 42 (2006), pp. 221-322.

[FrdI] S. Mochizuki, The Geometry of Frobenioids I: The General Theory, Kyushu J. Math. 62 (2008), pp. 293-400.

[FrdII] S. Mochizuki, The Geometry of Frobenioids II: Poly-Frobenioids, Kyushu J. Math. 62 (2008), pp. 401-460.

[EtTh] S. Mochizuki, The Étale Theta Function and its Frobenioid-theoretic Manifestations, Publ. Res. Inst. Math. Sci. 45 (2009), pp. 227-349.

[AbsTopIII] S. Mochizuki, Topics in Absolute Anabelian Geometry III: Global Reconstruction Algorithms, RIMS Preprint 1626 (March 2008).

[IUTchI] S. Mochizuki, Inter-universal Teichmüller Theory I: Construction of Hodge Theaters, preprint.

[IUTchII] S. Mochizuki, Inter-universal Teichmüller Theory II: Hodge-Arakelov-theoretic Evaluation, preprint.

[IUTchIV] S. Mochizuki, Inter-universal Teichmüller Theory IV: Log-volume Computations and Set-theoretic Foundations, preprint. 